\title{
Point Source Approximation Methods in Inverse Obstacle Reconstruction Problems
}

\author{
Dissertation \\ zur Erlangung des Doktorgrades \\ der Mathematisch-Naturwissenschaftlichen Fakultäten \\ der Georg-August-Universität zu Göttingen
}

\author{
vorgelegt von \\ Klaus Erhard \\ aus Bad Kissingen
}

Göttingen 2005 
D7

Referent:

Prof. Dr. R. Potthast

Korreferent:

Prof. Dr. R. Kress

Tag der mündlichen Prüfung: 7. November 2005 


\section{Contents}

\section{Part I Preliminaries}

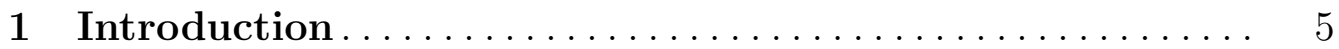

$2 \quad$ Some Useful Tools $\ldots \ldots \ldots \ldots \ldots \ldots \ldots \ldots \ldots \ldots \ldots \ldots \ldots \ldots$

2.1 Green's Theorems ............................. 11

2.2 Fundamental Solutions $\ldots \ldots \ldots \ldots \ldots \ldots \ldots \ldots \ldots \ldots \ldots$

2.3 Laplace's Equation ................................ 15

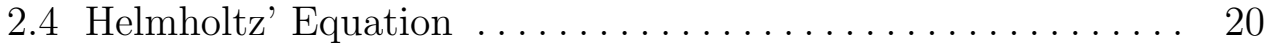

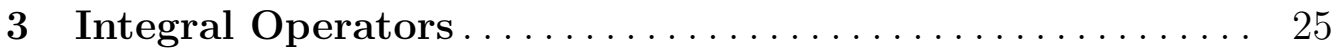

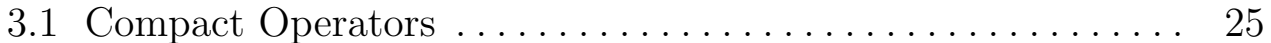

3.2 Layer Potentials ................................. 30

3.3 Nyström's Method .............................. 38

\section{Part II Main Tools}

4 Direct Boundary Value Problems .................. 47

4.1 Numerical Implementation of the Layer Potential Operators . 47

4.2 A Dirichlet Boundary Value Problem ................ 52

5 Point Source Approximation .................... 65

5.1 Approximation with a Single-Layer Potential ............ 65

5.2 Approximation with the Herglotz Wave Operator. . . . . . . . . 74

5.3 Choice of Approximation Domains ................. 80

\section{Part III Reconstruction Schemes}

$6 \quad$ The Point Source Method $\ldots \ldots \ldots \ldots \ldots \ldots \ldots \ldots \ldots$

6.1 The PSM for Boundary Value Problems.............. 91

6.2 The PSM for Acoustic Scattering Problems . . . . . . . . . . . . 105

6.3 Numerical Examples ................................. 114 
7 The Probe Method ... . . . . . . . . . . . . . . . . . . . . . 141

7.1 Linear Sampling and Probe Methods . . . . . . . . . . . . . . 142

7.2 The Probe Method of Ikehata . . . . . . . . . . . . . . . 143

7.3 Numerical Implementation of the Probe Method .......... 150

7.4 Numerical Examples ... . . . . . . . . . . . . . . . . . . . . . . . . 154

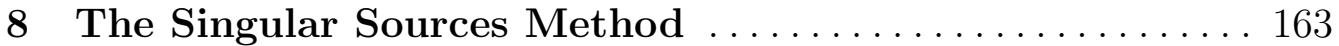

8.1 The SSM in Inverse Scattering . . . . . . . . . . . . . . . . . 163

8.2 A Comparison of Sampling Methods . . . . . . . . . . . . . . 172

8.3 Numerical Examples of the SSM in $\mathbb{R}^{3} \ldots \ldots \ldots \ldots \ldots$

References ................................ 203

Index . . . . . . . . . . . . . . . . . . . . . . 207 
Part I

Preliminaries 

Summary. In this thesis, we consider the point source method and related algorithms for the reconstruction of a sound-soft obstacle $D$ with acoustic waves. The core of the point source method consists of an approximation of the point source $\Phi(\cdot, z)$ on an approximation domain $G(z)$ and provides an approximation to the scattered field whenever $\bar{D} \subset G(z)$ holds. However, the decision whether the admissibility condition $\bar{D} \subset G(z)$ is satisfied was based on heuristics up to now. We close this gap and present an indicator function which yields an approximation to this admissibility region.

So far, the point source method was limited to reconstruct only one part of the obstacle's boundary. We remedy this drawback with the help of the new indicator function for the admissibility region. To this end we consider several fixed configurations $\left(z^{(j)}, G\left(z^{(j)}\right)\right.$ of the source point $z^{(j)}$ and its approximation domain $G\left(z^{(j)}\right)$ for $j=1, \ldots, N$. Then for each $j=1, \ldots, N$ we obtain a single reconstruction of the scattered field using translations of the fixed reference configuration $\left(z^{(j)}, G\left(z^{(j)}\right)\right.$ along a fixed translation direction. After restricting each single reconstruction to its admissibility region with the indicator function we obtain a complete reconstruction on the union of the single admissibility regions by an averaging procedure. We demonstrate that the reconstruction can be improved by the choice of adapted approximation domains in a two-step strategy.

Furthermore we reformulate the point source method for an inverse boundary value problem in electric impedance tomography. We introduce special static Herglotz wave functions of the Laplace equation to speed-up the point source method in the static case. With the approximation techniques of the point source method we also give a numerical realization of Ikehata's probe method. Together with the work of Cheng et al. [4] this is the first numerical study of the probe method. Finally, we apply the new techniques to the singular sources method and compare this reconstruction scheme with the linear sampling and the factorization method. In particular, we present a numerical study on these methods in $\mathbb{R}^{2}$ with noisy data up to an error of $20 \%$. All reconstruction schemes are illustrated with numerical examples in $\mathbb{R}^{2}$. For the point source method and the method of singular sources we additionally provide numerical examples in $\mathbb{R}^{3}$. 



\section{Introduction}

In many applications from medical imaging, nondestructive testing and geophysical exploration, the main goal is to gain insight into a region $\mathcal{B}$ that is not accessible by direct observation. Therefore one is interested to gain additional information about this area by measurements of physical quantities outside or on the boundary of the domain of interest. In this work we consider the problem to reconstruct an inclusion that differs significantly from the background medium, which we assume to be of a homogeneous physical nature. More precisely, we will focus on acoustic scattering problems, where we search for a sound-soft inclusion by measurements of the scattering response due to an incident acoustic wave. The ideas presented herein can be carried over to inverse electromagnetic scattering problems in a straightforward way, see [54] and [12].

In 1996 Potthast proposed the point source method which was developed in a series of papers [52], [53], [56]. This method belongs to the class of decomposition methods in inverse scattering, see [57], since it solves the nonlinear and ill-posed inverse shape reconstruction problem by a decomposition into a linear ill-posed problem and a nonlinear well-posed problem. In the first ill-posed step the PSM reconstructs the scattered field from the far field pattern with a linear backprojection operator. With this knowledge the PSM solves the nonlinear but well-posed problem to find the zeros of the total field (Dirichlet boundary condition) or its normal derivative (Neumann boundary condition) in a second step. Hence the point source method can also be regarded as an analytic continuation method extending the far field of a scattered wave to its near field.

The main idea of this method is to approximate the point source $\Phi(\cdot, z)$ on an approximation domain $G(z)$ with a Herglotz wave function $v_{z}$ and to use its kernel $g_{z}$ for the reconstruction of the scattered field in $z$. However, the reconstructed value approximates the true scattered field only if the unknown obstacle $D$ is contained in the approximation domain $G(z)$. Potthast suggested to use a fixed configuration $(z, G(z))$ of source point and corresponding approximation domain which is moved over the whole region $\mathcal{B}$ by translations and rotations. With this technique he obtained an efficient 
algorithm that reconstructs the scattered field whenever the admissibility condition $\bar{D} \subset G(z)$ is satisfied. A determination of this admissibility region has not been pursued, yet. Therefore, only partial reconstructions dependent on the translation direction of the configuration $(z, G(z))$ have been obtained in this manner.

We pick up this topic and present an indicator for the admissibility region. Then, we use this additional information for a complete reconstruction of the obstacle. We will also see that a proper choice of the approximation domain improves the reconstruction significantly. Hence we introduce a two-step strategy, where we use rather general approximation domains in a first reconstruction step, which are than adapted to the shape of the approximated admissibility region in the final reconstruction.

Furthermore, we illustrate the capability of the point source method by applying it to an inverse boundary value problem in electric impedance tomography. Since the point source method was originally formulated for inverse scattering problems we present a redesign of this method for acoustic boundary value problems first. Here the total field $u$ together with its normal derivative is given on a measurement boundary instead of the far field pattern of the scattered wave. Additionally, we introduce static Herglotz wave functions for the approximation of the fundamental solution of Laplace's equation. Similar to the acoustic case we then can take advantage of the translation invariance of the approximation domains in order to speed up the algorithm.

Apart from the class of decomposition methods other reconstruction schemes known as sampling and probe methods have been developed. The basic idea behind these methods is to construct an indicator function which is sampled on the unknown area. Then, the shape of the obstacle can be reconstructed from the behaviour of this indicator function at the obstacle's boundary or in its interior. The probe method was contributed by Ikehata [21] to this category of reconstruction schemes. Its indicator function is based on an approximation of the point source $\Phi(\cdot, z)$ on suitable approximation domains. We demonstrate that the approximation techniques of the point source method can be applied to the probe method as well. In particular, we define an indicator function for the admissibility region and show complete reconstructions of the obstacle.

Other sampling methods such as the linear sampling and the factorization method do not use these approximation techniques and may serve for a comparison with the point source approximation methods. To this end we choose the singular sources method as an example of this class of reconstruction schemes since it is defined in the same framework as the linear sampling and the factorization method. Again, we deduce an indicator for the admissibility region and provide an algorithm that yields a complete reconstruction of the obstacle. A numerical study demonstrates that the 
singular sources method is comparable with the linear sampling and the factorization method both without noise and with noise-affected data.

This work is structured into three parts. The first part contains a brief introduction into the underlying partial differential equations and some general tools for solving linear integral equations. Chapter 2 is dedicated to the partial differential equations under consideration. The Laplace and the Helmholtz equation together with some boundary value problems that will be used in the following are introduced. We also summarize Green's representation theorems for these partial differential equations. In Chapter 3 we deal with integral equations of the first and of the second kind. We focus on compact integral operators and recall the theory of Riesz and Fredholm as well as the method of Nyström for the numerical solution of integral equations of the second kind.

The second part of this thesis provides specific tools for solving the direct boundary value problems and for the approximation of the point source on its approximation domain. First, we study the direct problems under consideration in Chapter 4 and present numerical schemes for their solution. To this end we introduce a numerical implementation of the two-dimensional layer operators based on a separation of the logarithmic singularity. The forward solvers of this chapter will then be used to produce the input data for the reconstruction schemes in the last part. Chapter 5 explains the point source approximation techniques in detail. We show that a single-layer potential on the measurement boundary $\partial \Omega$ can also be used for the point source approximation instead of a Herglotz wave function. For the Laplace equation we present static Herglotz wave functions, which have been suggeted by Nakamura in private communications. With these functions we demonstrate that the efficiency boost due to the translation invariance of the configurations $(z, G(z))$ is applicable in the static case, too.

These approximation techniques build the main part of the reconstruction schemes that are discussed in the last part. Here, the point source method, the probe method and the singular sources method are presented in a formulation which allows a complete reconstruction with the help of an indicator function for the admissibility region and a weighting operation. In Chapter 6 we develop a redesign of the point source method for boundary value problems. In this situation we do not measure the far field pattern which describes the asymptotical behaviour of the scattered field far away from the obstacle, but we measure the Cauchy data of the total field on a measurement boundary $\partial \Omega$ of a domain $\Omega$ containing the scatterer. The point source method will be deduced with Green's theorem without using the reciprocity relation and can therefore be applied to any far field pattern and not only those which arise from incident plane waves. We illustrate the flexibility of the PSM by applying it to an inverse boundary value problem in electrostatics before we turn our interest to the inverse acoustic scattering problem. We demonstrate that the PSM for inverse scattering problems arises from 
the PSM for boundary value problems by considering large measurement boundaries and letting the radius of these measurement boundaries tend to infinity. Furthermore we provide a new characterization of the admissibility region in terms of an indicator function which relates the point source method with the no response test, see [43] and [58]. To obtain a complete reconstruction from the single reconstructions with a fixed reference configuration $(z, G(z))$ we proceed as follows. First, we use the indicator function of the admissibility region to restrict each single reconstruction to its admissibility region. Then we apply an averaging procedure in the overlapping part of the union of the single admissibility regions. This operation yields a complete reconstruction of the total field which we demonstrate with several numerical examples both for the inverse boundary value and the scattering problem. We show that the reconstruction can be improved by the choice of adapted approximation domains in a two-step strategy.

Chapter 7 presents the probe method in its original formulation of Ikehata and its numerical realization as demonstrated in [13]. In particular we develop a numerical realizable algorithm for the probe method in the same manner as demonstrated for the point source method, i.e. we apply the same approximation techniques and a similar indicator function for the admissibility region to obtain a complete reconstruction of the obstacle. To study the feasibility of the probe method we will use two equivalent formulations of Ikehata's indicator function and evaluate the true limiting function numerically. We will study the numerical realization of the functional proposed by Ikehata and show numerical reconstructions of soundsoft obstacles with the probe method. Together with the work of Cheng et al. [4] this is the first numerical realization of the probe method. Finally, we apply these approximation techniques to the singular sources method in Chapter 8. Additionally, we give a short overview of the linear sampling and the factorization method, which we consider for a comparison with the method of singular sources. We study the stability of these reconstruction schemes with respect to data noise in the two-dimensional inverse acoustic scattering problem. Furthermore, we provide numerical examples of the singular sources method in the inverse three-dimensional acoustic scattering problem.

Finally, I would like to thank my supervisor, Professor Roland Potthast for introducing me to the point source method and for a lot of fruitful discussions on this thesis. I am grateful to Professor Rainer Kress for carefully reading the manuscript and his valuable suggestions for improvement. Thanks do also go to Professor Gen Nakamura for our discussion on the probe method and his static Herglotz wave functions. 
To my parents 



\section{Some Useful Tools}

The starting point for many reconstruction algorithms based on point source approximation and hence for our summary of useful tools, are Green's formulae and Green's representation theorem. We will first introduce Green's formulae, which we prove with the divergence theorem of Gauss and then, we will have a look at two of the arguably most famous partial differential equations of mathematical physics, namely the Laplace and the Helmholtz equation. We present Green's representation theorem for harmonic functions and its analogon for solutions of the Helmholtz equation. Since we will consider obstacle reconstruction problems for both partial differential equations we will provide some short introduction on them in the following sections.

\subsection{Green's Theorems}

Throughout this work we will consider open and bounded subsets of $\mathbb{R}^{m}, m=2,3$ with some regularity condition on the boundary.

Definition 2.1. Let $D$ be an open bounded subset of $\mathbb{R}^{m}, m=2,3$. The boundary $\partial D$ is called of class $C^{r}, r \in \mathbb{N}$, if there exists a finite open cover

$$
\bar{D} \subset \bigcup_{k=1}^{n} V_{k}
$$

of $\bar{D}$, such that to every set $V_{l}$ with $V_{l} \cap \partial D \neq \emptyset$ there exists a bijective mapping $\varphi_{l}: V_{l} \cap \bar{D} \rightarrow H$ that maps $V_{l}$ onto the upper half ball

$$
H:=\left\{x \in \mathbb{R}^{m}:|x|<1, x_{m} \geq 0\right\} .
$$

In addition we demand $\varphi_{l}$ and its inverse $\varphi_{l}^{-1}$ to be $r$-times continuously differentiable and that $\varphi$ maps the set $V_{l} \cap \partial D$ onto $H \cap\left\{x \in \mathbb{R}^{m}: x_{m}=0\right\}$.

We will now present the divergence theorem of Gauss for bounded domains with boundary of class $C^{1}$. Historically, the divergence theorem of 
Gauss goes back to the work Theoria attractionis corporum sphaeroidicorum ellipticorum homogeneorum methodus nova tractata of Johann Carl Friedrich Gauss (1777-1855), which he published in the year 1813 in the Commentationes societatis regiae scientarum Gottingensis recentiores.

Theorem 2.2 (Divergence Theorem of Gauss). Let $D \subset \mathbb{R}^{m}, m=2,3$ be a bounded domain with boundary $\partial D$ of class $C^{1}$ and with unit normal vector $\nu$ directed into the exterior of $D$. Furthermore let $\mathbf{v}$ be a vector field in $C(\bar{D}) \cap C^{1}(D)$, then there holds

$$
\int_{D} \operatorname{div} \mathbf{v} d x=\int_{\partial D} \nu \cdot \mathbf{v} d s
$$

From the divergence theorem of Gauss we can easily deduce Green's first and second formula, which have their origin in the Essay on the application of mathematical analysis to the theories of electricity and magnetism published by George Green (1793-1841) in Nottingham in the year 1828.

Theorem 2.3 (Green's Formulae). Let $D \subset \mathbb{R}^{m}, m=2,3$ be a bounded domain with boundary of class $C^{2}$ and with unit normal vector $\nu$ directed into the exterior of $D$. Then, for functions $u \in C^{1}(\bar{D})$ and $v \in C^{2}(\bar{D})$ there holds Green's first formula

$$
\int_{D}(u \Delta v+\nabla u \cdot \nabla v) d x=\int_{\partial D} u \frac{\partial v}{\partial \nu} d s .
$$

If, in addition, $u \in C^{2}(\bar{D})$, then Green's second formula

$$
\int_{D}(u \Delta v-v \Delta u) d x=\int_{\partial D}\left(u \frac{\partial v}{\partial \nu}-v \frac{\partial u}{\partial \nu}\right) d s
$$

holds true.

Proof. To prove Green's first formula apply the divergence theorem to the vector field $\mathbf{v}=u \nabla v$. Interchanging the roles of $u$ and $v$ in Green's first formula and subtracting the resulting equations proves Green's second formula.

Obviously the regularity assumption on the boundary $\partial D$ of the bounded domain $D$ can be weakened since the proof of Green's formulae depends solely on the divergence theorem. Hence Green's formulae hold for each domain, where the divergence theorem can be validated. For a more detailed discussion and a more general formulation of the divergence theorem we refer to [28] and [46]. 


\subsection{Fundamental Solutions}

In the theory of partial differential equations fundamental solutions play an important role in the study of existence and regularity of solutions. Though we consider only the Helmholtz equation and its "static" relative the Laplace equation in this work we provide a rather extensive, general approach to the concept of fundamental solutions than just presenting a fundamental solution to these special partial differential equations under consideration. Accepting this little excursion will furnish us with munition to apply the reconstruction methods presented in the second part to partial differential equations different from the Laplace or the Helmholtz equation. For this reason we will leave the classical path for a moment and have a short look at weak solutions of partial differential equations.

Definition 2.4. Let $\Omega$ be a bounded domain. A linear functional $u$ on $C_{0}^{\infty}(\Omega)$ is called a distribution if for every compact set $K \subset \Omega$ there is a constant $C$ and an integer $N$ such that for all $\phi \in C_{0}^{\infty}(K)$ there holds

$$
|u(\phi)| \leq C \sum_{|\alpha| \leq N} \sup \left|\partial^{\alpha} \phi\right|
$$

The set of all distributions on $\Omega$ is denoted by $\mathcal{D}^{\prime}(\Omega)$.

The somehow artificial notation $\mathcal{D}^{\prime}(\Omega)$ instead of $C_{0}^{\infty \prime}(\Omega)$ goes back to Laurent Schwartz (1915-2002) and his Généralisation de la notion de fonction, de dérivation, de transformation de Fourier et applications mathématique et physiques which appeared in 1948. For this work on the theory of distribution the Fields Medal was presented to him in august 1950 .

We illustrate the previous definition with Dirac's $\delta_{a}$ distribution at a point $a \in \mathbb{R}^{m}$ given by

$$
\delta_{a}(\phi)=\phi(a), \quad \phi \in C_{0}^{\infty}\left(\mathbb{R}^{m}\right),
$$

which satisfies (2.6) with $N=0$ and $C=1$. In addition to his contributions to mathematics Dirac was also a great physicist working on quantum mechanics and relativity theory. In 1930 Paul Adrien Maurice Dirac (19021984) published The principles of quantum mechanics for which he was awarded the Nobel Prize for physics in 1933.

There is a standard folklore of Dirac stories, mostly revolving around Dirac saying exactly what he meant and no more. Once when someone, making polite conversation at dinner, commented that it was windy, Dirac left the table and went to the door, looked out, returned to the table and replied that indeed it was windy. It has been said in jest that his spoken vocabulary consisted of "Yes", "No", and "I don't know". Certainly when Chandrasekhar was explaining his ideas to Dirac he continually interjected "yes" then explained to Chandrasekhar that "yes" did not mean that he agreed with what he was saying, only that he wished him to continue. He once said:

I was taught at school never to start a sentence without knowing the end of it. 
This may explain much about his conversation, and also about his beautifully written sentences in his books and papers. 1

In the space $\mathcal{D}^{\prime}(\Omega)$ of distributions, one can generalize the rule of partial integration to define a distribution derivative.

Definition 2.5. Let $u \in \mathcal{D}^{\prime}(\Omega)$. Then the distribution derivative of $u$ is defined by

$$
\left(\partial_{k} u\right)(\phi):=-u\left(\partial_{k} \phi\right), \quad \phi \in C_{0}^{\infty}(\Omega) .
$$

Higher derivatives can be defined by induction. Obviously, every distribution on $\Omega$ has a distribution derivative up to any order. Hence, we can give the following definition of a fundamental solution of a differential operator.

Definition 2.6. A distribution $E \in \mathcal{D}^{\prime}\left(\mathbb{R}^{m}\right), m=2,3$ is called a fundamental solution of the differential operator $L=\sum a_{\alpha} \partial^{\alpha}$ with constant (complex) coefficients $a_{\alpha}$ if $L E=\delta_{0}$.

As an example, we present a fundamental solution for the Laplace equation.

Example 2.7. Consider the differential operator $L=\Delta=\partial_{1}^{2}+\partial_{2}^{2}$ on the space $\mathcal{D}^{\prime}\left(\mathbb{R}^{2}\right)$. Then a fundamental solution of the differential operator $L$ is given by

$$
E(\phi):=\frac{1}{2 \pi} \int_{\mathbb{R}^{2}} \log |x| \phi(x) d x, \quad \phi \in C_{0}^{\infty}\left(\mathbb{R}^{2}\right) .
$$

Proof. The integral over the unbounded domain $\mathbb{R}^{2}$ on the right hand side of (2.9) reduces to an integration over a bounded domain since each $\phi \in C_{0}^{\infty}\left(\mathbb{R}^{2}\right)$ has compact support. Furthermore, after a change to polar coordinates and due to the integrability of $\log r$ on $[0,1]$ the right hand side of $(2.9)$ is well defined.

The linear functional $E$ satisfies (2.6) with $N=0$ and the constant

$$
C_{K}=\frac{1}{2 \pi} \int_{K}|\log | x|| d x
$$

therefore $E$ is a distribution.

The fact that $\Delta \log |x|=0$ in $\mathbb{R}^{2} \backslash\{0\}$ together with Green's second formula yields

$$
\begin{aligned}
(L E)(\phi) & =E(L \phi)=\frac{1}{2 \pi} \int_{\mathbb{R}^{2}} \log |x| \Delta \phi(x) d x \\
& =\lim _{\varepsilon \rightarrow 0} \int_{B_{\varepsilon, R}}\left(\frac{1}{2 \pi} \log |x| \Delta \phi(x)-\phi(x) \Delta \frac{1}{2 \pi} \log |x|\right) d x \\
& =\lim _{\varepsilon \rightarrow 0} \int_{S_{\varepsilon}}\left(-\frac{1}{2 \pi} \log |x| \nabla \phi(x) \cdot \frac{x}{|x|}+\phi(x) \frac{1}{2 \pi} \frac{1}{|x|}\right) d s .
\end{aligned}
$$

\footnotetext{
${ }^{1}$ From the "MacTutor history of mathematics archive" on the web site

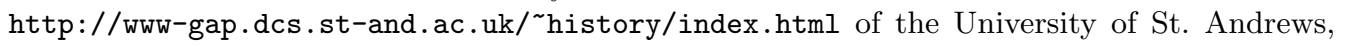
Scotland
} 
Since the first integral vanishes and the second integral converges to $\phi(0)$ when $\varepsilon \rightarrow 0$ we finally obtain $L E=\delta_{0}$ and $E$ is identified as a fundamental solution of $\Delta$.

Analogously one proves that

$$
E(\phi):=\frac{1}{4 \pi} \int_{\mathbb{R}^{3}} \frac{-1}{|x|} \phi(x) d x, \quad \phi \in C_{0}^{\infty}\left(\mathbb{R}^{3}\right)
$$

is a fundamental solution of the differential operator $L=\Delta=\partial_{1}^{2}+\partial_{2}^{2}+\partial_{3}^{2}$ in $\mathcal{D}^{\prime}\left(\mathbb{R}^{3}\right)$.

The existence of a fundamental solution for partial differential equations with constant coefficients is provided by the Malgrange-Ehrenpreis theorem.

Theorem 2.8 (Malgrange-Ehrenpreis). Every differential operator $L$ with constant coefficients has a fundamental solution.

For the proof we refer the reader to Theorem 1.56 in [15]. In particular the theorem of Malgrange-Ehrenpreis proves the existence of a fundamental solution to the differential operator $L=\Delta+\kappa^{2}$ with $\kappa \in \mathbb{C}$. In [69] Laurent Schwartz deduced the fundamental solution

$$
E(\phi)=\int_{\mathbb{R}^{m}} k(x) \phi(x) d x
$$

with the kernel function

$$
k(x)=\left\{\begin{array}{cc}
\frac{i}{4} H_{0}^{(1)}(\kappa|x|), & m=2, \\
\frac{1}{4 \pi} \frac{\mathrm{e}^{i \kappa|x|}}{|x|}, & m=3
\end{array}\right.
$$

for the Helmholtz equation with the help of the Fourier transform. Here $H_{0}^{(1)}$ denotes the Hankel function of first kind and order zero. A summary of fundamental solutions for various differential operators can be found in [50] while we refer to [49] and [51] for details on the construction of fundamental solutions.

\subsection{Laplace's Equation}

Newton's theory of gravitation is one of the most famous areas of application of the Laplace equation. Sir Isaac Newton (1643-1727) published his Principia, namely the Philosophiae naturalis principia mathematica in the year 1687 where he formulated the law of gravitation that "all matter attracts all other matter with a force proportional to the product of their masses and inversely proportional to the square of the distance between them". In 
mathematical terms the law of gravitation states that one point $x_{1} \in \mathbb{R}^{3}$ of mass $m_{1}$ attracts another point $x_{2} \in \mathbb{R}^{3}$ of mass $m_{2}$ with the force

$$
F_{12}\left(x_{2}\right)=-\gamma m_{1} m_{2} \frac{x_{1}-x_{2}}{\left|x_{1}-x_{2}\right|^{3}},
$$

where $\gamma$ denotes the gravitational constant. To define the gravitational field of the point mass $m_{1}$, which should be independent on the mass of the test body $m_{2}$, we can normalize the force $F_{12}$ by the mass of the test body. Hence we can define the gravitational field of a point $x_{1}$ with mass $m_{1}$ as

$$
G(x)=\frac{F_{12}(x)}{m_{2}}=-\gamma m_{1} \frac{x_{1}-x}{\left|x_{1}-x\right|^{3}}, \quad x \in \mathbb{R}^{3} \backslash\left\{x_{1}\right\} .
$$

The gravitational field $G$ can be expressed by the gradient of the potential

$$
u(x)=-\gamma m_{1} \frac{1}{\left|x_{1}-x\right|}, \quad x \in \mathbb{R}^{3} \backslash\left\{x_{1}\right\},
$$

of the point mass $m_{1}$, i.e. $G(x)=\nabla u(x)$. Thus the potential $u$ is a solution of Laplace's equation $\Delta u=0$ in $\mathbb{R}^{3} \backslash\left\{x_{1}\right\}$ and therefore the problem of finding the gravitational field of a system of bodies is closely related to the problem of finding solutions to the Laplace equation.

Sir Isaac Newton was knighted in 1705 by Queen Anne. He was the first scientist who was honoured for his work in this way. But despite of his great genius he is also famous for his raging dispute with Leibniz over the invention of the calculus in his last period of life.

Hundred years after Newton's Principia Charles Augustin de Coulomb (1736-1806) developed a theory explaining the repulsion and the attraction of point charges with the same or opposite electrical charge similar to the action of gravity on two mass points. Coulomb's law describing the force $F_{12}$ of an electrical charge $q_{1}$ at $x_{1}$ on a second electrical charge $q_{2}$ at point $x_{2}$ is of the same structure as the law of gravitation (2.14). We have to replace the point masses $m_{1}, m_{2}$ by the point charges $q_{1}, q_{2}$ and the gravitational constant by the constant $1 / 4 \pi \varepsilon_{0}$ and obtain

$$
F_{12}\left(x_{2}\right)=-\frac{1}{4 \pi \varepsilon_{0}} q_{1} q_{2} \frac{x_{1}-x_{2}}{\left|x_{1}-x_{2}\right|^{3}}
$$

with the electric permittivity $\varepsilon_{0}$ of free space. Hence the corresponding electrical potential $u$ of a point charge $q_{1}$ located in $x_{1}$ is defined analogously to (2.16) by

$$
u(x)=-\frac{1}{4 \pi \varepsilon_{0}} q_{1} \frac{1}{\left|x_{1}-x\right|}, \quad x \in \mathbb{R}^{3} \backslash\left\{x_{1}\right\}
$$

and its gradient $\nabla u$ generates the electric field due to this point charge. Again the problem of finding the electrical field of a system of charged bodies leads us to the Laplace equation and its solutions. 
Besides these historical application in gravitation and electrostatics the Laplace equation occurs in a variety of different areas, for example in the steady-state heat flow, the flow of irrotational fluids in hydrodynamics and in elasticity problems to mention a few.

Definition 2.9. A twice continuously differentiable, real-valued function u on a domain $D \subset \mathbb{R}^{m}$ that satisfies Laplace's equation

$$
\Delta u=0
$$

in $D$ is called harmonic.

A whole bunch of examples for harmonic functions in $\mathbb{R}^{2}$ are set up by the real and imaginary part of holomorphic functions in $\mathbb{C}$, which follows easily from the Cauchy-Riemann differential equations.

We will now introduce the fundamental solution of the Laplace equation and remark that we distinguish between the notion of a fundamental solution of the Laplace operator and the fundamental solution of the Laplace equation. While the first one is not unique and has to be understood in a distributional sense, the latter is defined as a family of particular classical solutions to the Laplace equation which are parametrized by its second argument $y \in \mathbb{R}^{m}$ and solve the Laplace equation in all of $\mathbb{R}^{m}$ except for the singular point $x=y$.

Definition 2.10. The function

$$
\Phi(x, y):=\left\{\begin{array}{cc}
\frac{1}{2 \pi} \log \frac{1}{x-y}, & m=2 \\
\frac{1}{4 \pi} \frac{1}{|x-y|}, & m=3
\end{array}\right.
$$

defined for all $(x, y) \in \mathbb{R}^{m} \times \mathbb{R}^{m} \backslash\{(x, y): x=y\}$ is called fundamental solution of Laplace's equation.

The function $\Phi(\cdot, y)$ is easily seen to be harmonic in $\mathbb{R}^{m} \backslash\{y\}$. We remark that $-\Phi(\cdot, 0)$ rather than $\Phi(\cdot, 0)$ generates a fundamental solution of the Laplace operator in the distributional sense, while $\Phi(\cdot, 0)$ can be regarded as a distributional fundamental solution to the negative Laplacian. This inconsistency in the notation is due to historical reasons and resembles the notation in the standard literature.

Starting with Green's representation theorem we will summarize some important properties of harmonic functions.

Theorem 2.11 (Green's Representation Formula). Let $D \subset \mathbb{R}^{m}$ be $a$ bounded domain with boundary of class $C^{2}$ and exterior unit normal vector $\nu$. If $u \in C^{2}(\bar{D})$ is harmonic in $D$ then Green's representation formula

$$
u(x)=\int_{\partial D}\left(\Phi(x, y) \frac{\partial u}{\partial \nu}(y)-\frac{\partial \Phi(x, y)}{\partial \nu(y)} u(y)\right) d s(y)
$$

holds for all $x \in D$. 
Proof. Let $x \in D$. Then there exists an $\varepsilon$-neighbourhood $B_{1}:=B_{\varepsilon}(x)$ of $x$ and a sequence $\left(\phi_{n}\right) \subset C_{0}^{\infty}\left(B_{1}\right)$ such that

$$
\phi_{n} \rightarrow u, \quad n \rightarrow \infty
$$

in $C^{2}\left(\overline{B_{2}}\right)$ with $B_{2}:=B_{\varepsilon / 2}(x)$. We assume the unit normal vector $\nu$ to the boundary $\partial B_{2}$ to be directed into the interior of the ball $B_{2}$. From

$$
\begin{aligned}
& \int_{B_{2}} \Phi(x, y) \Delta \phi_{n}(y) d y \\
& =\int_{B_{1}} \Phi(x, y) \Delta \phi_{n}(y) d y-\int_{B_{1} \backslash B_{2}} \Phi(x, y) \Delta \phi_{n}(y) d y \\
& =\int_{\mathbb{R}^{m}} \Phi(x, y) \Delta \phi_{n}(y) d y-\int_{B_{1} \backslash B_{2}}\left(\Phi(x, y) \Delta \phi_{n}(y)-\phi_{n}(y) \Delta_{y} \Phi(x, y)\right) d y \\
& =-\phi_{n}(x)-\int_{\partial B_{2}}\left(\Phi(x, y) \frac{\partial \phi_{n}}{\partial \nu}(y)-\frac{\partial \Phi(x, y)}{\partial \nu(y)} \phi_{n}(y)\right) d s(y)
\end{aligned}
$$

follows by taking the limit $n \rightarrow \infty$

$$
\int_{B_{2}} \Phi(x, y) \Delta u(y) d y=-u(x)-\int_{\partial B_{2}}\left(\Phi(x, y) \frac{\partial u}{\partial \nu}(y)-\frac{\partial \Phi(x, y)}{\partial \nu(y)} u(y)\right) d s(y) .
$$

Since $\Delta u=0$ in $B_{2}$ the left hand side of the above equation vanishes and an application of Green's second formula in the domain $D \backslash B_{2}$ yields

$$
0=-u(x)+\int_{\partial D}\left(\Phi(x, y) \frac{\partial u}{\partial \nu}(y)-\frac{\partial \Phi(x, y)}{\partial \nu(y)} u(y)\right) d s(y)
$$

which proves the theorem.

Corollary 2.12. Harmonic functions are analytic.

For a detailed proof of this corollary we refer the reader to Theorem 6.6 in [36].

Theorem 2.13 (Strong Maximum-Minimum Principle). A nonconstant harmonic function defined on a domain $D \subset \mathbb{R}^{m}$ can neither assume its maximum nor its minimum in D.

The proof of the strong maximum-minimum principle is provided for example in [17]. The uniqueness proof for the classical Dirichlet problem for Laplace's equation in bounded domains is based on the next corollary, which is an immediate consequence of the strong maximum-minimum principle.

Corollary 2.14 (Weak Maximum-Minimum Principle). Let $D$ be a bounded domain and $u \in C^{2}(\bar{D})$ be harmonic in $D$. Then $u$ attains both its maximum and its minimum on the boundary $\partial D$. 
We finish the discussion of Laplace's equation with a summary of its most common boundary value problems. For this purpose we consider a bounded domain $D \subset \mathbb{R}^{m}$ with a connected boundary $\partial D$ of class $C^{2}$ and with the unit normal $\nu$ directed into the exterior of $D$.

Interior Dirichlet Problem. Find a function $u \in C^{2}(D) \cap C(\bar{D})$ which is harmonic in $D$ and satisfies the boundary values

$$
u=f \quad \text { on } \partial D
$$

where $f$ is a given continuous function.

Interior Neumann Problem. Find a function $u \in C^{2}(D) \cap C(\bar{D})$ which is harmonic in $D$ and whose normal derivative satisfies the boundary values

$$
\frac{\partial u}{\partial \nu}=g \quad \text { on } \partial D
$$

in the sense

$$
\lim _{h \rightarrow+0} \nu \cdot \nabla u(x-h \nu(x))=g(x), \quad x \in \partial D,
$$

of uniform convergence on $\partial D$ where $g$ is a given continuous function.

Exterior Dirichlet Problem. Find a function $u \in C^{2}\left(\mathbb{R}^{m} \backslash \bar{D}\right) \cap C\left(\mathbb{R}^{m} \backslash D\right)$ which is harmonic in $\mathbb{R}^{m} \backslash \bar{D}$ and satisfies the boundary values

$$
u=f \quad \text { on } \partial D
$$

where $f$ is a given continuous function. In addition for $|x| \rightarrow \infty$ it is required that

$$
u(x)=\left\{\begin{array}{cc}
O(1), & m=2 \\
o(1), & m=3
\end{array}\right.
$$

uniformly for all directions $x /|x|$.

Exterior Neumann Problem. Find a function $u \in C^{2}\left(\mathbb{R}^{m} \backslash \bar{D}\right) \cap C\left(\mathbb{R}^{m} \backslash\right.$ D) which is harmonic in $\mathbb{R}^{m} \backslash \bar{D}$ and whose normal derivative satisfies the boundary values

$$
\frac{\partial u}{\partial \nu}=g \quad \text { on } \partial D
$$

in the sense of uniform convergence on $\partial D$ where $g$ is a given continuous function. In addition for $|x| \rightarrow \infty$ it is required that $u(x)=o(1)$ uniformly for all directions $x /|x|$.

The problem of uniqueness of the above boundary value problems is studied in Theorems 6.10 and 6.11 of [36]. We summarize the results in the following theorem. 
Theorem 2.15. Both the interior and the exterior Dirichlet problem as well as the exterior Neumann problem have at most one solution. Two solutions of the interior Neumann problem can differ only by a constant.

\subsection{Helmholtz' Equation}

While Laplace's equation is connected with the equilibrium steady state of a physical system we will now turn to a time-dependent physical process the propagation of acoustic waves in a homogeneous isotropic medium. The acoustic wave can be described by the pressure $p$ and the velocity field $v$ in the medium at any time $t$ which are related via the velocity potential $U$ by

$$
v(x, t)=\frac{1}{\varrho_{0}} \nabla_{x} U(x, t), \quad p(x, t)=-\frac{\partial}{\partial t} U(x, t)
$$

with the density $\varrho_{0}$ of the medium. Then the velocity potential $U$ satisfies the wave equation

$$
\frac{\partial^{2}}{\partial t^{2}} U-c^{2} \Delta_{x} U=0
$$

where $c$ is the speed of sound in the media.

We will restrict ourselves to the time-harmonic case where one is interested in solutions of the form

$$
U(x, t)=\operatorname{Re}\left(u(x) \mathrm{e}^{-i \omega t}\right)
$$

to the wave equation, i.e. we assume that the acoustic wave is generated by some oscillating sound source emitting sound waves with a fixed frequency $f=\omega / 2 \pi$. Inserting $(2.33)$ in $(2.32)$ yields

$$
-\omega^{2} u(x) \mathrm{e}^{-i \omega t}-c^{2} \Delta u(x) \mathrm{e}^{-i \omega t}=0
$$

and therefore with $\kappa=\omega / c$ the wave equation reduces for time-harmonic solutions to

$$
\Delta u+\kappa^{2} u=0
$$

the so called reduced wave equation or Helmholtz equation. More general, if we admit the medium to be dissipative, then the velocity potential satisfies the dissipative wave equation

$$
\frac{\partial^{2}}{\partial t^{2}} U+\gamma \frac{\partial U}{\partial t}-c^{2} \Delta U=0
$$

with a damping coefficient $\gamma$. In this case time-harmonic solutions (2.33) have to satisfy the reduced wave equation (2.35) with $\kappa$ such that $\kappa^{2}=$ $\omega(\omega+i \gamma) / c^{2}$ and $\operatorname{Im} \kappa \geq 0$. In the following we will refer to $\kappa$ as the wave number and for fixed $d \in \mathbb{S}^{m-1}$ we will call the function 


$$
u(x)=\mathrm{e}^{i \kappa x \cdot d}, \quad x \in \mathbb{R}^{m}
$$

a plane wave with direction $d$. Obviously, plane waves are solutions to the Helmholtz equation in $\mathbb{R}^{m}$.

We have already seen that the distribution (2.12) with the kernel function (2.13) defines a fundamental solution for the Helmholtz operator $L=\Delta+\kappa^{2}$. This motivates the following definition.

Definition 2.16. The function

$$
\Phi(x, y):=\left\{\begin{array}{rl}
\frac{i}{4} H_{0}^{(1)}(\kappa|x-y|), & m=2, \\
\frac{1}{4 \pi} \frac{\mathrm{e}^{i \kappa|x-y|}}{|x-y|}, & m=3,
\end{array} \quad x \neq y\right.
$$

is called the fundamental solution for the Helmholtz equation. Here, $H_{0}^{(1)}$ denotes the Hankel function (also known as the Bessel function of third kind) of the first kind of order zero.

Analogously to Green's representation Theorem 2.11 we can now formulate and prove a representation for solutions to the Helmholtz equation, which is often referred to as the Helmholtz representation theorem.

Theorem 2.17 (Helmholtz' Representation Theorem). Let $D \subset \mathbb{R}^{m}$ be a bounded domain with boundary of class $C^{2}$ with unit normal vector $\nu$ to the boundary $\partial D$ directed into the exterior of $D$. Any solution $u \in C^{2}(\bar{D})$ to the Helmholtz equation

$$
\Delta u+\kappa^{2} u=0 \quad \text { in } D,
$$

can be represented in $D$ with its Cauchy data on $\partial D$ in the form

$$
u(x)=\int_{\partial D}\left(\Phi(x, y) \frac{\partial u}{\partial \nu}(y)-\frac{\partial \Phi(x, y)}{\partial \nu(y)} u(y)\right) d s(y), \quad x \in D .
$$

As in the case of Laplace's equation we will need some additional decay condition at infinity for solutions to the exterior boundary value problems for the Helmholtz equation to guarantee uniqueness.

Definition 2.18. Let $u$ be defined on the exterior of some bounded domain in $\mathbb{R}^{m}$ where it is a solution to the Helmholtz equation. Then, we say that $u$ satisfies the Sommerfeld radiation condition if

$$
\lim _{|x| \rightarrow \infty}|x|^{\frac{m-1}{2}}\left(\frac{\partial u}{\partial r}-i \kappa u\right)=0, \quad m=2,3,
$$

holds uniformly for all directions $x /|x|$. 
Arnold Johannes Wilhelm Sommerfeld (1868-1951) was born in Königsberg, Prussia. After completing his dissertation at the University of Königsberg he went to Göttingen which he considered to be the seat of mathematical high culture during that time. There he became assistant of Felix Klein (18491925) in September 1894, who had a strong influence on Sommerfeld. It was Klein who taught him the importance of an intensive interrelation between mathematics and physics. Nowadays, Sommerfeld is mainly known for his contributions to theoretical physics. During his professorship on theoretical physics at Munich he supervised nearly 30 doctoral students. Among them, there are such famous Nobel prize winners such as Werner Heisenberg, Wolfgang Pauli, Hans Albrecht Bethe and Peter Debye.

Sommerfeld presented the radiation condition at the Annual Meeting of Natural Scientists in Münster 1912 to prove uniqueness for exterior boundary value problems for the Helmholtz equation. It was published in the paper Die Greensche Funktion der Schwingungsgleichung [70] the same year. Sommerfeld additionally required a "finiteness condition" on the field $u$ in this work, namely the boundedness of ru. However, in 1943 Franz Rellich (1906-1955) showed in [64] that the finiteness condition is superfluous in the formulation of the exterior boundary value problem. Rellich also generalized Sommerfeld's radiation condition for arbitrary dimensions and stated his well known lemma to prove uniqueness of the exterior boundary value problem in this publication. For a complete history of the radiation condition we refer the reader to the paper [68] of Schot.

With the Sommerfeld radiation condition we are able to formulate the boundary value problems for the Helmholtz equation similar to those for Laplace's equation.

Interior Dirichlet Problem. Find a function $u \in C^{2}(D) \cap C(\bar{D})$ which is a solution to the Helmholtz equation in $D$ satisfying the boundary values

$$
u=f \quad \text { on } \partial D
$$

where $f$ is a given continuous function.

Interior Neumann Problem. Find a function $u \in C^{2}(D) \cap C(\bar{D})$ satisfying the Helmholtz equation in $D$ and whose normal derivative satisfies the boundary values

$$
\frac{\partial u}{\partial \nu}=g \quad \text { on } \partial D
$$

in the sense

$$
\lim _{h \rightarrow+0} \nu \cdot \nabla u(x-h \nu(x))=g(x), \quad x \in \partial D,
$$

of uniform convergence on $\partial D$ where $g$ is a given continuous function. 
Exterior Dirichlet Problem. Find a function $u \in C^{2}\left(\mathbb{R}^{m} \backslash \bar{D}\right) \cap C\left(\mathbb{R}^{m} \backslash\right.$ D) which is a solution to the Helmholtz equation in $\mathbb{R}^{m} \backslash \bar{D}$ satisfying the Sommerfeld radiation condition and the boundary values

$$
u=f \quad \text { on } \partial D
$$

where $f$ is a given continuous function.

Exterior Neumann Problem. Find a solution $u \in C^{2}\left(\mathbb{R}^{m} \backslash \bar{D}\right) \cap C\left(\mathbb{R}^{m} \backslash\right.$ $D)$ to Helmholtz' equation in $\mathbb{R}^{m} \backslash \bar{D}$ satisfying Sommerfeld's radiation condition at infinity and the boundary values

$$
\frac{\partial u}{\partial \nu}=g \quad \text { on } \partial D
$$

in the sense of uniform convergence where $g$ is a given continuous function.

In contrast to the interior Dirichlet problem for Laplace's equation we do not have uniqueness for the corresponding boundary value problem for the Helmholtz equation. We demonstrate this fact with the following example.

Example 2.19. Let $\kappa>0$ and consider the domain

$$
D:=\left\{x \in \mathbb{R}^{3}: \frac{\pi}{\kappa}<|x|<\frac{2 \pi}{\kappa}\right\} .
$$

Then the function

$$
u(x):=\frac{\sin \kappa|x|}{|x|}
$$

is a solution to the Helmholtz equation in $D$ with $u=0$ on $\partial D$. Hence, there is a non-trivial solution to the interior Dirichlet problem for the Helmholtz equation with vanishing boundary data. In this case $-\kappa^{2}$ is called a Dirichlet eigenvalue of the Laplacian in D. Neumann eigenvalues are defined analogously.

In view of example 2.19 the uniqueness Theorem 2.15 for the boundary value problems of the Laplace equation will have to be modified for Helmholtz' equation.

Theorem 2.20. The exterior Dirichlet and Neumann problems have at most one solution. If $\operatorname{Im} \kappa>0$ the same holds true for the interior Dirichlet and Neumann problems.

For the proof we refer the reader to Theorem 3.13 and Theorem 3.10 in [10]. We mention that uniqueness of the interior Dirichlet (Neumann) problem can be forced by the assumption that $-\kappa^{2}$ is not a Dirichlet (Neumann) eigenvalue of the Laplacian. 



\section{Integral Operators}

The aim of this section is to provide a little toolbox for solving integral equations of the first and of the second kind. To this end we first introduce compact operators with an emphasis on integral operators with a continuous or weakly singular kernel. In the study of equations of the first kind we first encounter the notion of ill-posedness and we introduce the concept of regularization schemes for the approximative solution of equations of the first kind. In this context we summarize the Tikhonov regularization technique as one of its most famous representative. Next we focus on layer potentials and give a short summary of their properties. For further use we state the theorem of Riesz and Fredholm's alternative in dual systems. Finally we present Nyström's method for the numerical treatment of integral equations of the second kind.

\subsection{Compact Operators}

In the following we study linear equations of the form

$$
A x=y
$$

and

$$
x-B x=y
$$

with linear operators $A: X \rightarrow Y$ from a normed space $X$ into a normed space $Y$ and a linear self-mapping $B: X \rightarrow X$ of the normed space $X$ respectively. More precisely we assume these linear operators to be compact.

Definition 3.1. A subset $U$ of a normed space $X$ is called relatively compact if its closure is compact in $X$. A linear operator $A: X \rightarrow Y$ from a normed space $X$ into a normed space $Y$ is called compact if it maps any bounded set in $X$ into a relatively compact set in $Y$. Furthermore (3.1) is said to be an equation of the first kind if the operator $A: X \rightarrow Y$ is compact. Equation (3.2) is called an equation of the second kind if the operator $B: X \rightarrow X$ is compact. 
We provide some examples for compact operators in terms of integral operators $A: C(\partial D) \rightarrow C(\partial D)$ given by

$$
(A \varphi)(x)=\int_{\partial D} k(x, y) \varphi(y) d s(y), \quad x \in \partial D
$$

with a suitable kernel $k: \partial D \times \partial D \rightarrow \mathbb{C}$. Here the integration domain $\partial D$ corresponds to the boundary of some bounded domain $D \subset \mathbb{R}^{m}$. We give some sufficient conditions on the kernel $k$ such that the integral operator (3.3) is compact. In particular continuity of the kernel $k$ on $\partial D \times \partial D$ implies compactness of the integral operator $A$. Note that all proofs of the following theorems can be looked up for example in the book [33].

Theorem 3.2. The integral operator $A$ defined by (3.3) with continuous kernel is a compact operator on $C(\partial D)$.

This standard result can be improved to hold for integral operators with a weakly singular kernel.

Definition 3.3. Let the kernel

$$
k: \partial D \times \partial D \backslash\{(x, x): x \in \partial D\} \rightarrow \mathbb{C}
$$

be continuous and satisfy

$$
|k(x, y)| \leq C|x-y|^{\alpha-(m-1)},
$$

for all $x, y \in \partial D, x \neq y$ with a positive constant $C$ and $\alpha \in(0, m-1]$. Then $k$ is called $a$ weakly singular kernel.

Theorem 3.4. The integral operator A defined by (3.3) with weakly singular kernel is a compact operator on $C(\partial D)$.

We will give some explicit examples of compact integral operators with a weakly singular kernel in the next section, where we investigate the layer potential opertors. For the time being we consider the integral equation of the first kind (3.1) with a compact integral operator $A: X \rightarrow Y$ defined between two normed spaces $X$ and $Y$. This kind of equation arises for example in the problem of approximating the fundamental solution $\Phi(\cdot, z)$ as demonstrated in Chapter 5.

If the range of $A$ is of infinite dimension, then the compact operator $A$ can not possess a bounded inverse, i.e. there does not exist a continuous inverse mapping $A^{-1}: Y \rightarrow X$ in this case. Assuming the contrary we obtain the compactness of the operator $B=A A^{-1}$ since it is a product of the compact operator $A$ and the bounded operator $A^{-1}$. On the other hand $B$ equals the identity on the range $\{y \in Y: \exists x \in X$ s.t. $A x=y\}$ of the operator $A$. But this contradicts the fact that the identity on a normed space of infinite dimension is not compact. 
Hence the solution to equation (3.1) does not depend continuously on the data given by the right hand side. From a numerical point of view it is impossible to calculate a solution to (3.1) directly since any small round-off error in the computation leads to a perturbation of the data and due to the discontinuity of the inverse operator we have to expect an inpredictable and unreasonable calculated solution to (3.1). This problem is ill-posed in the sense of Hadamard. He suggested to call a problem well-posed if

- there exists a solution to the problem,

- the solution to the problem is unique, and

- the solution is continuously dependent on the data.

In contrary, we call a problem ill-posed if it is not well-posed, that is if the problem lacks at least one of the three properties proposed by Hadamard. if a problem does not possess at least one of the properties stated above we call it ill-posed.

In 1923 Jacques Salomon Hadamard (1865-1963) introduced the concept of well-posedness for initial value and boundary value problems in his lectures on Cauchy's problem in linear partial differential equations at the University of Yale. Besides his contribution to partial differential equations of mathematical physics he proved the prime number theorem which states that the number of primes less than $n$ tends to infinity as $n / \log n$ in 1896 .

From the three criteria of ill-posedness the first two ones can be remedied for a linear operator $A: X \rightarrow Y$ by considering the operator $\tilde{A}: X / N(A) \rightarrow A(X)$ mapping the space $X$ factorized by the null space $N(A)$ onto the image space $A(X)$ of $A$, i.e.

$$
\tilde{A}(x+N(A)):=A x, \quad x+N(A) \in X / N(A) .
$$

Though the characterization of the null space may be a hard task, the previous factorization is always, at least theoretically, possible without changing the operator $A$.

But, if the solution of a problem does not depend continuously on the data then there is no chance of calculating the solution directly since any small perturbation of the data regardless whether it originates from an error in the measured data or a computational error may lead to an unstable solution. Therefore a violation of the third critertion of Hadamard is the major manifestation of ill-posedness which we have to overcome. If we are aware of this ill-posedness of the problem we do not get around a regularization of the solution in order to find a stable approximate solution to the solution of the original problem.

Definition 3.5. A familiy of bounded linear operators

$$
R_{\alpha}: Y \rightarrow X, \quad \alpha>0
$$

is called a regularization scheme for the operator $A: X \rightarrow Y$ if the operator sequence $\left(R_{\alpha} A\right)$ converges pointwise to the identity on $X$, i.e. if 


$$
\lim _{\alpha \rightarrow 0} R_{\alpha} A x=x
$$

for all $x \in X$. We call the parameter $\alpha$ the regularization parameter.

The ill-posedness of (3.1) with a compact operator on an infinite dimensional space reveals in a corresponding regularization scheme $R_{\alpha}$ in the fact that neither can the operators $R_{\alpha}$ be bounded uniformly nor does the sequence $\left(R_{\alpha} A\right)$ converge in the operator norm to the identity.

Theorem 3.6. Let $R_{\alpha}: Y \rightarrow X$ be a regularization scheme for the compact operator $A: X \rightarrow Y$ defined on an infinite dimensional space $X$. Then there exists a sequence $\left(\alpha_{k}\right)$ with $\lim _{k \rightarrow 0} \alpha_{k}=0$ and $\left\|R_{\alpha_{k}}\right\| \geq k$. Moreover, the sequence $\left(R_{\alpha} A x\right)$ does not converge in the operator norm to the identity $I: X \rightarrow X$.

Proof. We assume that the operators $R_{\alpha}$ are uniformly bounded, that is there exists a constant $C>0$ such that $\left\|R_{\alpha}\right\| \leq C$ for all $\alpha>0$. Let $y \in A(X)$ and $\varepsilon>0$, then we can find an $\alpha_{0}>0$ and an $x \in X$ with $y=A x$ such that

$$
\left\|R_{\alpha_{0}} y-x\right\|=\left\|R_{\alpha_{0}} A x-x\right\| \leq \varepsilon
$$

and we estimate

$$
\left\|A^{-1} y\right\|=\left\|x-R_{\alpha_{0}} A x+R_{\alpha_{0}} y\right\| \leq \varepsilon+C\|y\| .
$$

Since $\varepsilon>0$ was arbitrary we conclude that $A^{-1}$ is bounded and hence the identity $I=A^{-1} A$ on $X$ is compact, which contradicts the fact that $X$ is a space of infinite dimension.

We prove the second statement with an analogous argument. We assume that the convergence $R_{\alpha} A \rightarrow I$ holds in the operator norm. Then the compactness of the operators $R_{\alpha} A$ carries over to the limit operator $I: X \rightarrow X$, which again contradicts the assumption on the dimension of $X$.

The reconstruction schemes are defined in a way such that the pointwise convergence $R_{\alpha} y \rightarrow A^{-1} y$ for $\alpha \rightarrow 0$ holds for all $y \in A(X)$. However, if the data $y$ is affected by noise and only the erroneous data $y^{\delta}$ with $\left\|y^{\delta}-y\right\| \leq \delta$ is available, then a careless choice of the regularization parameter will result in an "approximate" solution $x^{\delta}$ of the true solution $x$ of $A x=y$ which does not approximate the true solution at all. To make this phenomenon clear we have a look at the following splitting of the error between the true and the approximate solution:

$$
\left\|x^{\delta}-x\right\|=\left\|R_{\alpha} y^{\delta}-R_{\alpha} y+R_{\alpha} y-x\right\| \leq\left\|R_{\alpha}\right\| \delta+\left\|R_{\alpha} A x-x\right\| .
$$

Letting $\alpha$ tend to zero for a fixed error level $\delta$ leads to an exploding error between the true and the approximate solution due to the unboudedness 
of the regularization operators $R_{\alpha}$. In contrast, an increasing regularization parameter decreases the first part of the error, but then the second term $\left\|R_{\alpha} A x-x\right\|$, which describes the quality of the approximation of the inverse operator $A^{-1}$ by the regularizing operators $R_{\alpha}$, will become larger. Therefore a good choice of the regularization parameter should minimize the right hand side of (3.11). Since this choice depends on the value of the error level $\delta$ we demand for a good choice $\alpha(\delta)$ of the regularization parameter that $x_{\alpha(\delta)}^{\delta} \rightarrow x$ whenever $\delta \rightarrow 0$.

Definition 3.7. The choice of the regularization parameter $\alpha=\alpha(\delta)$ depending on the error level $\delta$ is called a strategy for the regularization scheme $R_{\alpha}, \alpha>0$. We call a strategy for a regularization scheme regular if

$$
\left\|R_{\alpha(\delta)} y^{\delta}-A^{-1} y\right\| \rightarrow 0, \quad \delta \rightarrow 0,
$$

holds for all $y \in A(X)$ and all $y^{\delta} \in Y$ with $\left\|y^{\delta}-y\right\| \leq \delta$.

There are several different strategies for the choice of the regularization parameter, which can be divided into the class of a priori and a posteriori strategies. The a priori strategies rely on some additional information on the problem, for example information about the regularity of the solution, and choose the regularization paramter in advance while the a posteriori strategies choose the parameter $\alpha$ during the regularization process of computing $x_{\alpha}^{\delta}$. For a complete survey of regularization schemes for inverse problems we refer to the monograph [31] and to the appropriate chapters in [36].

In the following we present the Tikhonov regularization, as a penalized residual minimization technique for the regularization of (3.1) with a compact operator $A$ between two Hilbert spaces. More generally, Tikhonov's regularization scheme can be deduced from the singular value decomposition of the compact operator $A$ and the introduction of a suitable damping function in Picard's theorem. We mention that this approach leads to a variety of different regularization schemes such as the spectral cut off and the Landweber scheme by a proper choice of the damping function, see [31] for details.

The Tikhonov regularization will serve as a regularization scheme for the ill-posed problem of approximating the fundamental solution by an element in the range of some compact integral operator. Thus we are interested in minimizing the residual $\|A x-y\|$ among all $x \in X$. In order to stabilize the minimization procedure we add a penalty term of the form $\alpha\|x\|$ with a regularization parameter $\alpha>0$ to the residual. The existence and uniqueness of a minimizing element in $X$ is stated in the following theorem.

Theorem 3.8. Let $X, Y$ be Hilbert spaces and let $A: X \rightarrow Y$ be a bounded linear operator. For each $y \in Y$ and $\alpha>0$ there exists a unique solution for the minimization problem

$$
\min _{x \in X}\|A x-y\|^{2}+\alpha\|x\|^{2}
$$


which is given by the unique solution of the equation

$$
A^{*} A x+\alpha x=A^{*} y .
$$

Tikhonov's regularization scheme $R_{\alpha}: Y \rightarrow X$ is then given by

$$
R_{\alpha}=\left(\alpha I+A^{*} A\right)^{-1} A^{*}
$$

and any strategy $\alpha(\delta)$ with $\alpha(\delta) \rightarrow 0$ and $\frac{\delta^{2}}{\alpha(\delta)} \rightarrow 0$ for $\delta \rightarrow 0$ is a regular strategy for Tikhonov's regularization scheme, see for example Corollary 15.27 in [36].

\subsection{Layer Potentials}

From electrostatics we know that an electric charge $q_{1}$ at the point $x_{1}$ exerts an electric force $F_{12}$, given by (2.17), on any test charge $q_{2}$ at position $x_{2}$. If we normalize the force $F_{12}$ on the test charge by its strength we obtain the electric field

$$
E(x)=\frac{F_{12}(x)}{q_{2}}=-\frac{1}{4 \pi \varepsilon_{0}} q_{1} \frac{x_{1}-x}{\left|x_{1}-x\right|^{3}}, \quad x \in \mathbb{R}^{3} \backslash\left\{x_{1}\right\}
$$

of a point charge $q_{1}$ in analogy to the definition (2.15) of the gravitational field of a point mass. In accordance with the gravitational potential the electric potential

$$
u(x)=-\frac{1}{4 \pi \varepsilon_{0}} q_{1} \frac{1}{\left|x_{1}-x\right|}, \quad x \in \mathbb{R}^{3} \backslash\left\{x_{1}\right\}
$$

is a solution to the Laplace equation in $\mathbb{R}^{3} \backslash\left\{x_{1}\right\}$ and it holds

$$
E(x)=\nabla u(x), \quad x \in \mathbb{R}^{3} \backslash\left\{x_{1}\right\} .
$$

The last equation states that the electric field of a point charge is generated by a potential which is a multiple of the fundamental solution of Laplace's equation $\Phi\left(x_{1}, x\right)$. In the same way $\Phi\left(x_{1}, x\right)$ is a generator for the gravitational field of a point mass in the sense that the gradient of $\Phi$ defines the gravitational field of a point mass up to a multiplicative factor. For this reason we will refer to the function $\Phi(x, y)$ also as a point source. In particular, if we consider $\Phi(x, y)$ for fixed $y \in \mathbb{R}^{m}$ as function of $x \in \mathbb{R}^{m}$ we call $\Phi(x, y)$ the point source with source point $y$. Due to the symmetry $\Phi(x, y)=\Phi(y, x)$ of the fundamental solution for all $x, y \in \mathbb{R}^{m}, x \neq y$ we denote the function $\Phi(y, \cdot)$ defined on $\mathbb{R}^{m} \backslash\{y\}$ also as the point source with source point $y$.

Since each point source solves the corresponding partial differential equation everywhere except in the source point, we could try to find solutions to the boundary value problems in the form of a superposition of point sources located on the boundary of the domain of interest. If we assume the point sources to be continuously distributed over the boundary we can define a single-layer potential as the superposition of the source point distribution over the boundary. 
Definition 3.9. Let $D \subset \mathbb{R}^{m}$ be a bounded domain with boundary of class $C^{2}$ and let $\varphi \in C(\partial D)$. Then we call

$$
u(x):=\int_{\partial D} \Phi(x, y) \varphi(y) d s(y), \quad x \in \mathbb{R}^{m} \backslash \partial D,
$$

the single-layer potential with density $\varphi$.

Besides a superposition of point sources in the form (3.19) the following superposition of continuously distributed dipoles over the boundary is useful.

Definition 3.10. Let $D \subset \mathbb{R}^{m}$ be a bounded domain with boundary of class $C^{2}$ and let $\varphi \in C(\partial D)$. Then we call

$$
v(x):=\int_{\partial D} \frac{\partial \Phi(x, y)}{\partial \nu(y)} \varphi(y) d s(y), \quad x \in \mathbb{R}^{m} \backslash \partial D,
$$

the double-layer potential with density $\varphi$.

An electric dipole is a configuration of two point charges with opposite sign $\pm q$ located at a finite distance $d$ and with the dipole moment $\mathbf{M}=q \mathbf{l}$, where $\mathbf{l}$ is the direction vector from the negative to the positive charge of length $|\mathbf{l}|=d$. Hence, at any point $y \in \partial D$ we can position a dipole of unit strength $|\mathbf{M}|=1 \mathrm{Cm}$ by locating a positive charge of strength $|\mathbf{M}| / 2 h$ at $y+h \nu(y)$ and a negative charge of the same strength at $y-h \nu(y)$. The potential of the dipole configuration is given by

$$
u(x)=-\frac{1}{2 h \varepsilon_{0}} \Phi(x, y+h \nu(y))+\frac{1}{2 h \varepsilon_{0}} \Phi(x, y-h \nu(y))
$$

for all $x \notin\{y-h \nu(y), y+h \nu(y)\}$ and letting the dipole length $2 h$ tend to zero, the corresponding potential of a unit dipole of infinitesimal length is

$$
u(x)=-\frac{1}{\varepsilon_{0}} \lim _{h \rightarrow 0} \frac{\Phi(x, y+h \nu(y))-\Phi(x, y-h \nu(y))}{2 h}=-\frac{1}{\varepsilon_{0}} \frac{\partial \Phi(x, y)}{\partial \nu(y)},
$$

for $x \in \mathbb{R}^{m} \backslash\{y\}$. Therefore the double-layer potential is a superposition of continuously distributed dipoles on the boundary $\partial D$. The name doublelayer reflects the construction of an electric dipole layer as two layers of a point source distribution on parallel surfaces to $\partial D$ with opposite sign and infinitesimal distance.

For points $x \notin \partial D$ we can differentiate both the single- and the doublelayer potential under the integral and therefore the layer potentials are solutions to their underlying partial differential equation in $\mathbb{R}^{m} \backslash \partial D$. This property qualifies the layer potentials as ansatz functions for the presented interior boundary value problems. A study of the asymptotical behaviour of a point source and its derivatives when the argument tends to infinity 
reveals their capability in solving the exterior boundary value problems, too. Hence, the behaviour of the layer potentials at the boundary $\partial D$ is of special interest. We denote with $D_{-}=D$ the interior and with $D_{+}=\mathbb{R}^{m} \backslash \bar{D}$ the exterior domain and we define

$$
f_{ \pm}(x):=\lim _{h \rightarrow+0} f(x \pm h \nu(x)), \quad x \in \partial D
$$

for functions $f \in C\left(\mathbb{R}^{m} \backslash \partial D\right)$ which can be continuously extended from $D_{+}$ into $\bar{D}_{+}$and from $D_{-}$into $\bar{D}_{-}$respectively. Analogously we define

$$
\frac{\partial f_{ \pm}}{\partial \nu}(x):=\lim _{h \rightarrow+0}\langle\nabla f(x \pm h \nu(x)), \nu(x)\rangle, \quad x \in \partial D
$$

for functions $f \in C^{1}\left(\mathbb{R}^{m} \backslash \partial D\right)$ whenever the limit (3.24) exists in the sense of uniform convergence on $\partial D$. We summarize the limiting values of the layer potentials on $\partial D$ in the next theorem, see [36] and [10], which holds for both $\Phi$ denoting the fundamental solution of the Laplace and $\Phi$ being the fundamental solution of Helmhotz' equation.

Theorem 3.11. Let $D$ be a bounded domain with boundary $\partial D$ of class $C^{2}$ and $\varphi \in C(\partial D)$. Then the single-layer potential $u$ with continuous density $\varphi$ is continuous in $\mathbb{R}^{m}$ with the direct values

$$
u(x)=\int_{\partial D} \Phi(x, y) \varphi(y) d s(y), \quad x \in \partial D
$$

on the boundary and

$$
\frac{\partial u_{ \pm}}{\partial \nu}(x)=\int_{\partial D} \frac{\partial \Phi(x, y)}{\partial \nu(x)} \varphi(y) d s(y) \mp \frac{1}{2} \varphi(x), \quad x \in \partial D,
$$

where the integrals in (3.25) and (3.26) exist as improper integrals.

The double-layer potential $v$ with continuous density $\varphi$ can be extended continuously from $D_{-}$to $\bar{D}_{-}$and from $D_{+}$to $\bar{D}_{+}$with limiting values

$$
v_{ \pm}(x)=\int_{\partial D} \frac{\partial \Phi(x, y)}{\partial \nu(y)} \varphi(y) d s(y) \pm \frac{1}{2} \varphi(x), \quad x \in \partial D,
$$

where the integral in (3.27) exists as an improper integral. Furthermore, there holds

$$
\lim _{h \rightarrow+0}\left(\frac{\partial v}{\partial \nu}(x+h \nu(x))-\frac{\partial v}{\partial \nu}(x-h \nu(x))\right)=0, \quad x \in \partial D
$$

uniformly on $\partial D$.

The previous theorem connects the solvability of the boundary value problems for the Laplace and the Helmholtz equation in terms of some potential layer ansatz with the solvability of an integral equation on the boundary $\partial D$. Therefore it is convenient to introduce the following integral operators. 
Definition 3.12. Let $D \subset \mathbb{R}^{m}$ be a bounded domain with boundary of class $C^{2}$. Then we denote with $S: C(\partial D) \rightarrow C(\partial D)$ the integral operator

$$
(S \varphi)(x):=2 \int_{\partial D} \Phi(x, y) \varphi(y) d s(y), \quad x \in \partial D
$$

defined via the direct values of the single-layer potential on $\partial D$. Furthermore we define the integral operators $K: C(\partial D) \rightarrow C(\partial D)$ given by

$$
(K \varphi)(x):=2 \int_{\partial D} \frac{\partial \Phi(x, y)}{\partial \nu(y)} \varphi(y) d s(y), \quad x \in \partial D
$$

and $K^{\prime}: C(\partial D) \rightarrow C(\partial D)$ defined by

$$
\left(K^{\prime} \varphi\right)(x):=2 \int_{\partial D} \frac{\partial \Phi(x, y)}{\partial \nu(x)} \varphi(y) d s(y), \quad x \in \partial D
$$

via the direct values of the double-layer and the adjoint double-layer potential.

The potential layer operators give us examples of integral operators with a weak-singularity, however in the two-dimensional case the operators $K$ and $K^{\prime}$ even possess a continuous kernel.

Theorem 3.13. The operators $S, K$ and $K^{\prime}$ are compact in $C(\partial D)$.

Proof. We indicate the proof for the single-layer operator in the static case. The kernel of the single-layer operator for the Laplace equation is weaklysingular, since the estimate (3.5) holds for $m=2$ with $\alpha=1 / 2$ and for $m=3$ with $\alpha=1$. Hence the compactness of the operator $S$ follows from Theorem 3.4. Using the estimate

$$
|\langle\nu(x), x-y)| \leq L|x-y|^{2}
$$

for all $x, y \in \partial D$ with a suitable constant $L>0$, see Theorem 6.15 in [36], one can show that the integral operators $K$ and $K^{\prime}$ are weakly singular and thus compact. For the details we refer the reader to [36]. The proof of the statement in the acoustic case is given in [10], Theorem 2.30 and 2.31.

Besides the boundary integral operators for the direct values of the layer potentials it is helpful to define boundary integral operators for the evaluation of the layer potentials outside their domain of integration. In particular, for $n$ bounded domains with boundaries $\Gamma_{1}, \ldots, \Gamma_{n}$ of class $C^{2}$ which are disjoint by pairs, i.e. $\Gamma_{j} \cap \Gamma_{k}=\emptyset$ for $j \neq k$, we define the operator $S_{j k}: C\left(\Gamma_{k}\right) \rightarrow C\left(\Gamma_{j}\right)$ by

$$
\left(S_{j k} \varphi_{k}\right)(x):=2 \int_{\Gamma_{k}} \Phi(x, y) \varphi_{k}(y) d s(y), \quad x \in \Gamma_{j}
$$


and in an analogous way $K_{j k}$ and $K_{j k}^{\prime}$ for all $1 \leq j, k \leq n$.

For the further analysis of the solvability of integral equations of the second kind we now introduce some basic results from the theory of Fredholm and Riesz. Both Frigyes Riesz (1880-1956) and Erik Ivar Fredholm (18661927) are known as two of the founders of functional analysis and we will therefore turn our attention briefly towards the situation of the analysis at the turn of the century [74].

At this time, there have been two imortant schools of mathematicians the group of Henri Lebesgue (1875-1941) in Paris and the group of David Hilbert (1862-1943) in Göttingen. While the Paris school worked on Fourier series [40] and on circumventing the problems with the Riemann-integral which resulted in the upcoming Lebesgue-integral [39], Hilbert was dealing with integral equations and spectral theory [20]. The starting point of Fredholm's work is connected with the name of Jules Henri Poincaré (1854-1912) whom he met on a research stay in Paris. Poincaré considered the problem of a vibrating membrane, which leads to the well-established partial differential equation

$$
\Delta u+\lambda u=f
$$

with $u$ vanishing on the boundary of the membrane and an exterior excitation $f$. He transformed this boundary value problem into an integral equation of the form

$$
u(s)-\lambda \int_{a}^{b} k(s, t) u(t) d t=f(s)
$$

but without presenting a solution theory to this equation. In his investigations sur une classe d'équations fonctionelles (1900) Fredholm took up Poincaré's idea but left the special potential-theoretic problem aside and considered the integral equation (3.35) in its full generality. Led by the analogy to infinite systems of linear equations and based on the theory of infinite determinants he defined the determinant of the integral equation (3.35) and applied Cramer's rule. However, he rarely explained how to arrive at his determinant but it is known, see for example [11], that he probably first discretized (3.35) by a Riemann sum and considered the determinant of the resulting linear system. Using von Koch's theory of infinite determinants, but without taking much care on the limit process, he defined his determinant "in analogy with the case of a system of linear equations" by replacing sums with integrals. In this paper he formulated the first variant of his alternative which he completed three years later in [16] to the full extend.

In winter 1900/1901 David Hilbert got to know Fredholm's results in a Seminar in Göttingen from the Swedish mathematician Erik Holmgren (18731943) who was a student of Fredholm himself. Hilbert was immediately 
impressed of Fredholm's work and started to shift his research activities towards the arising field of integral equations which resulted in a series of papers between 1904 and 1910 and finally in his monograph [20] on the general theory of linear integral equations. He also explicitely performed the passage to the limit in Fredholm's proof (1904). His most important work, which Dieudonné [11] calls the very first paper on functional analysis, appeared as a note in 1906. In this work Hilbert imbedded the theory of integral equations into a more general theory by returning to the concept of infinite systems of linear equations. Instead of discretizing the integral equation and considering the limit of the resulting system of linear equations he reduced the solution theory of the integral equation (3.35) to that of a linear system with infinite many unknowns $a_{1}, a_{2}, a_{3}, \ldots$ with converging sum of squares $\sum_{k=1}^{\infty} a_{k}^{2}$.

The work of Hilbert together with Lebesgue's integration theory opened the door for Frigyes Riesz to appear on the stage. Riesz, and independently Ernst Fischer (1875-1959), proved in 1907 that Lebesgue's space $L^{2}$ of square-integrable functions and Hilbert's space $l^{2}$ of square-summable sequences are isomorphic. In his fundamental paper [65], which is reprinted in the collection [67], p. 441-489, he further introduced and discussed the $L^{p}$ spaces giving the first example of what we call today a reflexive Banach space which is not isomorphic to its dual, namely $L^{p}$ and its dual $L^{q}$ with $\frac{1}{p}+\frac{1}{q}=1$. His main results on spectral theory appeared in 1916 in Hungarian and were published two years later in German [66]. This work was the foundation of the Riesz-Fredholm theory which will be crucial for our further investigations, in particular the following theorem will be needed frequently.

Theorem 3.14. Let $X$ be a normed space and $A: X \rightarrow X$ a compact operator. Provided the operator $I-A$ is injective, the inverse operator (I$A)^{-1}: X \rightarrow X$ exists and is bounded.

It is convenient to reformulate the previous theorem for the appplication to integral equations of the second kind.

Corollary 3.15. Let $X$ be a normed space and $A: X \rightarrow X$ a compact operator. If the homogeneous equation

$$
\varphi-A \varphi=0
$$

admits only the trivial solution in $X$, then the inhomogeneous equation

$$
\varphi-A \varphi=f
$$

has a unique solution for any right hand side $f \in X$ on which it depends continuously.

For a complete history of functional analysis we refer the interested reader to Dieudonné's monograph [11] while the commemorative speech [34] of Rainer 
Kress on the occasion of the 65th birthday of Erich Martensen and Harro Heuser illustrates the history of Fredholm's alternative in dual system as we will present it in the following. To this end we first introduce the concept of dual systems.

Definition 3.16. Two normed spaces $X$ and $Y$ together with a non-degenerated bilinear form $\langle\cdot, \cdot\rangle: X \times Y \rightarrow \mathbb{C}$ are called a dual system for which we use the notation $\langle X, Y\rangle$.

Obviously, the bilinear form

$$
\langle\varphi, \psi\rangle:=\int_{\partial D} \varphi \psi d s, \quad \varphi, \psi \in C(\partial D)
$$

defines a dual system $\langle C(\partial D), C(\partial D)\rangle$ on $C(\partial D)$. Moreover, for the operators $K$ and $K^{\prime}$ there holds

$$
\langle K \varphi, \psi\rangle=\left\langle\varphi, K^{\prime} \psi\right\rangle
$$

for all $\varphi, \psi \in C(\partial D)$. If two operators are related with each other in this way we call them adjoint.

Definition 3.17. Let $\langle X, Y\rangle$ be a dual system. Two operators $A: X \rightarrow X$ and $B: Y \rightarrow Y$ are called adjoint with respect to the dual system $\langle X, Y\rangle$ if

$$
\langle A \varphi, \psi\rangle=\langle\varphi, B \psi\rangle
$$

holds for every $\varphi \in X, \psi \in Y$.

We have already seen that $K$ and $K^{\prime}$ are adjoint operators with respect to $\langle C(\partial D), C(\partial D)\rangle$ and due to their compactness the following variant of Fredholm's alternative holds for them.

Theorem 3.18 (Fredholm's alternative). Let $\langle X, Y\rangle$ be a dual system and $A: X \rightarrow X$ and $B: Y \rightarrow Y$ be compact adjoint operators. Then either

$$
N(I-A)=\{0\} \quad \text { and } \quad N(I-B)=\{0\}
$$

and

$$
(I-A)(X)=X \quad \text { and } \quad(I-B)(Y)=Y,
$$

or

$$
\operatorname{dim} N(I-A)=\operatorname{dim} N(I-B) \in \mathbb{N}
$$

and

$$
(I-A)(X)=\{f \in X:\langle f, \psi\rangle=0, \quad \psi \in N(I-B)\}
$$

and

$$
(I-B)(Y)=\{g \in Y:\langle\varphi, g\rangle=0, \quad \varphi \in N(I-A)\}
$$


The idea to use Fredholm's alternative in dual systems goes back to Wendland [76] and an elementary proof is given in [10], Theorem 1.30.

In the regularization scheme of Tikhonov we need to define the underlying compact operator on a Hilbert space. In particular the point source approximation is based on an integral equation of the first kind, which we have to consider on a Hilbert space such as $L^{2}(\Gamma)$ rather than on $C(\Gamma)$. Therefore we finish this section on layer potential operators with some remarks on the potentials defined in $L^{2}$ spaces. The next lemma proves that the image of a square integrable function under the operators $S_{j k}, K_{j k}$ and $K_{j k}^{\prime}$ is a continuous function. More generally, the following lemma holds:

Lemma 3.19. Let $M_{1}, M_{2} \subset \mathbb{R}^{m}$ be two compact Jordan measurable sets and the kernel $k(x, y): M_{1} \times M_{2} \rightarrow \mathbb{C}$ of the integral operator

$$
(A \varphi)(x):=\int_{M_{2}} k(x, y) \varphi(y) d s(y), \quad x \in M_{1}
$$

defined for square integrable densities $\varphi \in L^{2}\left(M_{2}\right)$, be continuous on $M_{1} \times$ $M_{2}$. Then $A \varphi \in C\left(M_{1}\right)$ for all $\varphi \in L^{2}\left(M_{2}\right)$.

Proof. Let $\varepsilon>0$. Then the compactness of $M_{1}$ and $M_{2}$ together with the continuity of $k$ implies the existence of a $\delta>0$ such that

$$
\left|k\left(x_{1}, y\right)-k\left(x_{2}, y\right)\right| \leq \varepsilon
$$

for all $y \in M_{2}$ and all $x_{1}, x_{2} \in M_{1}$ with $\left|x_{1}-x_{2}\right| \leq \delta$. Hence, an application of the Cauchy-Schwarz inequality yields

$$
\begin{aligned}
\left|(A \varphi)\left(x_{1}\right)-(A \varphi)\left(x_{2}\right)\right| & \leq \int_{M_{2}}\left|k\left(x_{1}, y\right)-k\left(x_{2}, y\right)\right||\varphi(y)| d s(y) \\
& \leq \varepsilon \int_{M_{2}}|\varphi(y)| d s(y) \\
& \leq \varepsilon C,
\end{aligned}
$$

with the constant

$$
C=\left(\int_{M_{2}} 1 d x\right)^{\frac{1}{2}}\|\varphi\|_{L^{2}\left(M_{2}\right)}
$$

When the layer potentials are defined on the Hilbert space $L^{2}(\Gamma)$ we have to reinvestigate the behaviour of the potentials near the boundary $\Gamma$. In this setting Kersten [29] extended the jump relations with the use of the Lax theorem from the case of continuous densities to $L^{2}$ densities. We summarize the results in the next theorem, where $\Phi$ is again to be understood as either the fundamental solution to the Laplace or to the Helmholtz equation and the integral operators $S, K$ and $K^{\prime}$ are defined as the extensions of the layer potential operators to the $L^{2}$ space. 
Theorem 3.20. Let $\Gamma$ be of class $C^{2}$ and $\varphi \in L^{2}(\Gamma)$. Then the single-layer potential

$$
u(x)=\int_{\Gamma} \Phi(x, y) \varphi(y) d s(y), \quad x \in \mathbb{R}^{m} \backslash \Gamma
$$

with square integrable density $\varphi \in L^{2}(\Gamma)$ admits the limits

$$
\lim _{h \rightarrow+0} \int_{\Gamma}|2 u(x \pm h \nu(x))-(S \varphi)(x)|^{2} d s(x)=0
$$

and

$$
\lim _{h \rightarrow+0} \int_{\Gamma}\left|2 \frac{\partial u}{\partial \nu}(x \pm h \nu(x))-\left(K^{\prime} \varphi\right)(x) \pm \varphi(x)\right|^{2} d s(x)=0 .
$$

Furthermore the double-layer potential

$$
v(x)=\int_{\Gamma} \frac{\partial \Phi(x, y)}{\partial \nu(y)} \varphi(y) d s(y), \quad x \in \mathbb{R}^{m} \backslash \Gamma
$$

with square integrable density $\varphi \in L^{2}(\Gamma)$ admits the limits

$$
\lim _{h \rightarrow+0} \int_{\Gamma}|2 v(x \pm h \nu(x))-(K \varphi)(x) \mp \varphi(x)|^{2} d s(x)=0
$$

and

$$
\lim _{h \rightarrow+0} \int_{\Gamma}\left|\frac{\partial v}{\partial \nu}(x+h \nu(x))-\frac{\partial v}{\partial \nu}(x-h \nu(x))\right|^{2} d s(x)=0 .
$$

Finally the layer potentials with square integrable densities turn out to define compact operators on the Hilbert space $L^{2}(\partial D)$.

Lemma 3.21. Let $D \subset \mathbb{R}^{m}$ be a bounded domain with boundary of class $C^{2}$. Then the integral operators $S: L^{2}(\partial D) \rightarrow L^{2}(\partial D), K: L^{2}(\partial D) \rightarrow L^{2}(\partial D)$ and $K^{\prime}: L^{2}(\partial D) \rightarrow L^{2}(\partial D)$ are compact operators.

This lemma is proved by approximating the weakly singular kernels of the layer potential under consideration with a sequence of Hilbert-Schmidt kernels, i.e. square integrable kernels. Then the resulting sequence of compact operators converges to the layer potential in the operator norm, which proves the compactness. For details we refer the reader to [75].

\subsection{Nyström's Method}

As we have seen in the previous section, the double-layer potential as well as the normal derivative of the single-layer potential has a jump discontinuity along the boundary $\partial D$. Using these potentials as ansatz functions for the 
Dirichlet and Neuman problems we have to solve an integral equation of the form

$$
\varphi(x)-\int_{\partial D} k(x, y) \varphi(y) d s(y)=f(x),
$$

since the ansatz functions satisfy the boundary values if and only if their densities solve the corresponding integral equation. The question of solvability has already been addressed with the theorem of Riesz. We will now study a numerical method - the Nyström method - to solve integral equations of the second kind.

Let $G \subset \mathbb{R}^{m}$ be a compact Jordan measurable set and

$$
Q_{n}(g):=\sum_{j=1}^{n} \alpha_{j}^{(n)} g\left(x_{j}^{(n)}\right), \quad n \in \mathbb{N}
$$

a sequence of quadrature rules for the integral

$$
Q(g):=\int_{G} w(x) g(x) d x
$$

with some weight function $w$. The numbers $\alpha_{1}^{(n)}, \ldots, \alpha_{n}^{(n)}$ are called quadrature weights and the points $x_{1}^{(n)}, \ldots, x_{n}^{(n)}$ are called quadrature points.

Definition 3.22. A sequence $\left(Q_{n}\right)$ of quadrature rules is called convergent, if $Q_{n}(g) \rightarrow Q(g)$ for $n \rightarrow \infty$ and all $g \in C(G)$.

Example 3.23. The repeated trapezoidal rule

$$
Q_{n}(g)=\sum_{j=0}^{n-1} \frac{h^{(n)}}{2}\left(g\left(x_{j+1}^{(n)}\right)+g\left(x_{j}^{(n)}\right)\right)
$$

with $h^{(n)}=(b-a) / n$ and $x_{j}^{(n)}=a+j h^{(n)}, j=0, \ldots, n$ is convergent towards the integral

$$
Q(g)=\int_{a}^{b} g(x) d x
$$

Especially for a periodic analytic function the application of the trapezoidal rule results in a remainder of order $O\left(\mathrm{e}^{-2 n s}\right)$, where the constant $s>0$ depends on the domain of analyticity of the integrand. More precisely the following theorem holds, see [36].

Theorem 3.24. Let $g: \mathbb{R} \rightarrow \mathbb{R}$ be an analytic $2 \pi$-periodic function. Then the error

$$
R(g):=\frac{1}{2 \pi} \int_{0}^{2 \pi} g(t) d t-\frac{1}{2 n} \sum_{j=0}^{2 n-1} g\left(\frac{j \pi}{n}\right)
$$

for the trapezoidal rule can be estimated by 


$$
|R(g)| \leq M(\operatorname{coth} n s-1)
$$

with a constant $M>0$ and with $s>0$ such that $g$ can be analytically extended to a holomorphic function on the strip $\mathbb{R} \times(-s, s)$ in the complex plane.

With the help of a convergent sequence $\left(Q_{n}\right)$ of quadrature rules we can approximate the integral operator $A: C(G) \rightarrow C(G)$ given by

$$
(A \varphi)(x)=\int_{G} k(x, y) \varphi(y) d y, \quad x \in G
$$

with continuous kernel $k \in C(G \times G)$ by the sequence of numerical integration operators

$$
\left(A_{n} \varphi\right)(x):=\sum_{k=1}^{n} \alpha_{k}^{(n)} k\left(x, x_{k}^{(n)}\right) \varphi\left(x_{k}^{(n)}\right), \quad x \in G
$$

obtained by applying the quadrature rules $Q_{n}$ for fixed $x \in G$ to the integrand $g(y)=k(x, y) \varphi(y)$. Then we can approximate the solution to the integral equation of the second kind

$$
\varphi-A \varphi=f
$$

by the solution of the approximate equation

$$
\varphi_{n}-A_{n} \varphi_{n}=f .
$$

Hence, substituting the quadrature $Q$ with the quadrature rules $Q_{n}$ gives rise to a discretization of the continuous problem (3.65). However the discretized approximation (3.66) to the continuous problem is still continuous in the first argument of the kernel. Therefore we call (3.66) a semi-discrete equation and in this context we call (3.66) a consistent discretization if $\left(Q_{n}\right)$ is a convergent sequence of quadrature formulae.

The solution to this semi-discrete equation can be obtained by solving a finite dimensional system of linear equations as stated by the next theorem, see [36].

Theorem 3.25. Let $\varphi_{n}$ be a solution to the semi-discrete equation

$$
\varphi_{n}(x)-\sum_{k=1}^{n} \alpha_{k}^{(n)} k\left(x, x_{k}^{(n)}\right) \varphi_{n}\left(x_{k}^{(n)}\right)=f(x), \quad x \in G .
$$

Then the values $\varphi_{j}^{(n)}=\varphi_{n}\left(x_{j}^{(n)}\right), j=1, \ldots, n$ at the the quadrature points satisfy the linear system

$$
\varphi_{j}^{(n)}-\sum_{k=1}^{n} \alpha_{k}^{(n)} k\left(x_{j}^{(n)}, x_{k}^{(n)}\right) \varphi_{k}^{(n)}=f\left(x_{j}^{(n)}\right), \quad j=1, \ldots, n .
$$


Conversely, if $\varphi_{j}^{(n)}, j=1, \ldots, n$ is a solution to the discrete system of linear equations (3.68) then the function $\varphi_{n}$ defined by

$$
\varphi_{n}(x):=f(x)+\sum_{k=1}^{n} \alpha_{k}^{(n)} k\left(x, x_{k}^{(n)}\right) \varphi_{k}^{(n)}, \quad x \in G
$$

solves the semi-discrete equation (3.67).

However, we still have to prove that if there exists a solution to the continuous problem then there also exists a solution to the approximate integral equation and that the solution $\varphi_{n}$ of this semi-discrete equation (3.66) converges to the true solution $\varphi$ of (3.65). Assuming the consistency of the discretization (3.66) the latter is true if there is an integer $N$ such that the inverse operators $\left(I-A_{n}\right)^{-1}$ exist and are uniformly bounded

$$
\left\|\left(I-A_{n}\right)^{-1}\right\| \leq C
$$

for all $n \geq N$ with a constant $C$. In this case we call the discretization (3.66) stable .

Theorem 3.26. Let the discretization (3.66) be consistent and stable. Then (3.65) is uniquely solvable in $C(G)$ and the discretization (3.66) is convergent towards (3.65), i.e. there exists an integer $N$ such that (3.66) is uniquely solvable for every $f \in C(G)$ and all $n \geq N$ and the limit

$$
\lim _{n \rightarrow \infty} \varphi_{n}=\varphi
$$

holds.

Proof. Lemma 4.1.10 in [19] proves that consistency and stability of the discretization implies the injectivity of the operator $I-A$. Then, unique solvability of the continuous problem follows from Riesz' Theorem 3.14 . Since the discretization is stable there exists an integer $N \in \mathbb{N}$ such that the inverse operators $\left(I-A_{n}\right)^{-1}$ exist and are uniformly bounded for all $n \geq N$. Finally we obtain

$$
\varphi_{n}-\varphi=\left(I-A_{n}\right)^{-1} f-\varphi=\left(I-A_{n}\right)^{-1}\left(A_{n}-A\right) \varphi
$$

and for $n \geq N$ we can derive the estimate

$$
\left\|\varphi_{n}-\varphi\right\| \leq C\left\|\left(A_{n}-A\right) \varphi\right\|
$$

which tends to zero for a consistent discretization.

To prove the convergence of the discretization (3.66) of Nyström's method we will show its consistency and stability. To this end we introduce the concept of a collectively compact set of operators. 
Definition 3.27. $A$ set $\mathcal{A}=\{A: X \rightarrow Y\}$ of linear operators mapping a normed space $X$ into a normed space $Y$ is called collectively compact if the image set $\mathcal{A}(U)=\{A \varphi: \varphi \in U, A \in \mathcal{A}\}$ of any bounded set $U \subset X$ is relatively compact in $Y$.

An example of a collectively compact set is provided by the numerical integration operators $\left(A_{n}\right)$ provided that the operators $A_{n}$ are defined via a convergent sequence of quadrature rules.

Theorem 3.28. The sequence of numerical integration operators $\left(A_{n}\right)$ given by (3.64) with a convergent sequence of quadrature formulae $\left(Q_{n}\right)$ defines a collectively compact set of operators.

This theorem is written down and proved in [36], Theorem 12.8. Based on the concept of collectively compact operator sequences we present an error analysis which goes back to Brakhage [3] and Anselone and Moore [2].

Theorem 3.29. Let $A: X \rightarrow X$ be a compact linear operator defined on a Banach space $X$. Furthermore, let $A_{n}: X \rightarrow X$ be a collectively compact sequence which is pointwise convergent towards $A$, i.e. $A_{n} \varphi \rightarrow A \varphi, n \rightarrow \infty$ for all $\varphi \in X$. Then

$$
\left\|\left(A_{n}-A\right) A\right\| \rightarrow 0, \quad n \rightarrow \infty
$$

and

$$
\left\|\left(A_{n}-A\right) A_{n}\right\| \rightarrow 0, \quad n \rightarrow \infty .
$$

If, in addition, $I-A$ is invertible, then for sufficiently large $n \in \mathbb{N}$ the operators $I-A_{n}$ are invertible and $\left\|\left(I-A_{n}\right)^{-1}\right\|$ is uniformly bounded. Furthermore, for the solution $\varphi$ of the equation (3.65) and $\varphi_{n}$ of the semidiscrete equation (3.66) hold the error estimates

$$
\left\|\varphi_{n}-\varphi\right\| \leq\left\|(I-A)^{-1}\right\| \frac{\left\|\left(A_{n}-A\right) f\right\|+\left\|\left(A_{n}-A\right) A_{n} \varphi\right\|}{1-\left\|(I-A)^{-1}\left(A_{n}-A\right) A_{n}\right\|}
$$

and

$$
\left\|\varphi_{n}-\varphi\right\| \leq\left\|\left(I-A_{n}\right)^{-1}\right\| \frac{\left\|\left(A_{n}-A\right) f\right\|+\left\|\left(A_{n}-A\right) A \varphi_{n}\right\|}{1-\left\|\left(I-A_{n}\right)^{-1}\left(A_{n}-A\right) A\right\|} .
$$

Proof. The first statement of the theorem is a reformulation of Corollary 10.7 in [33]. The invertibility of $I-A_{n}$ for large $n$ and the first error estimates follows from Theorem 10.8 in [33], which also ensures the existence of a uniform bound on the inverse operators $\left(I-A_{n}\right)^{-1}$. Hence we can finally apply Theorem 10.9 in [33] to prove the last error estimate.

Theorem 3.28 proves that the Nyström method with a convergent sequence of quadrature rules yields a consistent and stable discretization (3.66), provided that the continuous problem (3.65) is uniquely solvable. In view of Theorem 3.26 we can conclude the following convergence corollary. 
Corollary 3.30. Let the second kind integral equation (3.65) be uniquely solvable. Then the discretization (3.66) based on a convergent sequence of quadrature formulae is convergent towards (3.65).

Although, the error $\left\|\varphi-\varphi_{n}\right\|$ can be evaluated with the estimate (3.77), the computation of error bounds from (3.77) can be rather difficult. In contrast with (3.77) the error estimate (3.73) in the proof of Theorem 3.26 is based on the convergence order of the quadrature rules and depends on the quality of approximation of the integral operator $A$ by the numerical integration operators $A_{n}$. Therefore (3.73) is often more useful in practice. 

Part II

Main Tools 



\section{Direct Boundary Value Problems}

Before we can study any inverse problem we have to investigate the direct problem under consideration first. In particular, we will use the methods for solving the direct boundary value problems presented in this chapter to calculate the input data for the reconstruction problems in the last part of this work. To this end we introduce a numerical scheme for the two-dimensional layer potentials based on a separation of the logarithmic singularity. Moreover we revisit Nyström's method and apply it to integral equations involving integral operators with weakly singular kernels. We present a solution theory both for a direct boundary value problem for the Helmholtz and the Laplace equation. Finally we demonstrate numerical examples in the two-dimensional case.

\subsection{Numerical Implementation of the Layer Potential Operators}

Before we consider the direct boundary value problems we step into the numerics for second kind integral equations involving weakly singular kernels. We are especially interested in integral operators with a logarithmic singularity like the single-layer potential for both the Laplace and the Helmholtz equation. Hence we present a quadrature rule for the weakly singular operator

$$
(A \varphi)(t):=\frac{1}{2 \pi} \int_{0}^{2 \pi} \log 4 \sin ^{2} \frac{t-\tau}{2} k(t, \tau) \varphi(\tau) d \tau, \quad 0 \leq t \leq 2 \pi
$$

in the space of continuous periodic functions functions $C_{2 \pi}([0,2 \pi])$. We assume that the weak singularity of the kernel is of logarithmic type, i.e. the function $k$ is continuous on $[0,2 \pi] \times[0,2 \pi]$. Hence we need a quadrature rule for the improper integral

$$
(Q g)(t):=\frac{1}{2 \pi} \int_{0}^{2 \pi} \log 4 \sin ^{2} \frac{t-\tau}{2} g(\tau) d \tau,
$$


which can be constructed by approximating the continuous periodic function $g$ with its trigonometric interpolation polynomial and integrating the latter exactly.

With the equidistant quadrature points $t_{j}=j \pi / n, j=0, \ldots, 2 n-1$ the trigonometric interpolation operator $P_{n}$ is given by

$$
P_{n}(g):=\sum_{j=0}^{2 n-1} g\left(t_{j}\right) L_{j}^{(n)}
$$

with the Lagrange basis

$$
L_{j}^{(n)}(t)=\frac{1}{2 n}\left(1+2 \sum_{k=1}^{n-1} \cos k\left(t-t_{j}\right)+\cos n\left(t-t_{j}\right)\right)
$$

for $j=0, \ldots, 2 n-1$ and $0 \leq t \leq 2 \pi$. Inserting the trigonometric interpolation polynomial (4.3) for $g$ in (4.2) we obtain the quadrature rules

$$
\left(Q_{n} g\right)(t)=\sum_{j=0}^{2 n-1} R_{j}^{(n)}(t) g\left(t_{j}\right), \quad 0 \leq t \leq 2 \pi
$$

with the quadrature weights

$$
R_{j}^{(n)}(t):=\frac{1}{2 \pi} \int_{0}^{2 \pi} \log 4 \sin ^{2} \frac{t-\tau}{2} L_{j}(\tau) d \tau, \quad 0 \leq t \leq 2 \pi
$$

given by

$$
R_{j}^{(n)}(t)=-\frac{1}{n}\left(\sum_{m=1}^{n-1} \frac{1}{m} \cos m\left(t-t_{j}\right)+\frac{1}{2 n} \cos n\left(t-t_{j}\right)\right)
$$

for $j=0, \ldots, 2 n-1$ and $0 \leq t \leq 2 \pi$. Hence we define the numerical integration operators $A_{n}$ by an application of the quadrature rules $Q_{n}$ to the functions $k(t, \cdot) \varphi(\cdot)$, i.e.

$$
\left(A_{n} \varphi\right)(t):=\sum_{j=0}^{2 n-1} R_{j}^{(n)}(t) k\left(t, t_{j}\right) \varphi\left(t_{j}\right), \quad 0 \leq t \leq 2 \pi .
$$

An elaborate study of the numerical quadratures (4.5) as demonstrated in [33] yields the collectively compactness of the numerical integration operators $A_{n}$.

Theorem 4.1. The sequence of numerical integration operators (4.8) is collectively compact and pointwise convergent to the integral operator (4.1) with a logarithmic singularity. 
In particular, the last theorem states that the sequence (4.8) of numerical integration operators satisfy the conditions of Theorem 3.29. Hence the error estimates of Theorem 3.29 as well as Corollary 3.30 hold for Nyström's method applied to integral operators with a logarithmic singularity, too.

In the remaining part of this section we present the numerical implementation of the single- and double-layer potentials for both the Laplace and the Helmholtz equation with the delineated method. To this end we separate the logarithmic singularity of the two-dimensional single-layer potential.

Example 4.2 (The single-layer potential for the Laplace equation in 2D). We consider a bounded domain $D \subset \mathbb{R}^{2}$ with boundary of class $C^{2}$ and a periodic representation $x:[0,2 \pi] \rightarrow \partial D$ of this boundary with $x \in$ $C_{2 \pi}^{2}([0,2 \pi])$. Then, the direct values of the single-layer potential

$$
u(x(t))=\frac{1}{2 \pi} \int_{0}^{2 \pi} \log \frac{1}{|x(t)-x(\tau)|} \varphi(x(\tau))\left|x^{\prime}(\tau)\right| d \tau, \quad 0 \leq t \leq 2 \pi
$$

with continuous density $\varphi \in C(\partial D)$ admits the representation

$$
u(x(t))=-\frac{1}{4 \pi} \int_{0}^{2 \pi}\left(\log 4 \sin ^{2} \frac{t-\tau}{2}+\rho(t, \tau)\right) \varphi(x(\tau))\left|x^{\prime}(\tau)\right| d \tau
$$

with the continuous function

$$
\rho(t, \tau)=\left\{\begin{array}{cc}
\log \frac{|x(t)-x(\tau)|^{2}}{4 \sin ^{2} \frac{t-\tau}{2}}, & t \neq \tau \\
\log \left|x^{\prime}(t)\right|^{2} & , \quad t=\tau
\end{array}\right.
$$

in $C\left([0,2 \pi]^{2}\right)$.

Moreover the two-dimensional double-layer potential for the Laplace equation turns out to be continuous.

Example 4.3 (The double-layer potential for the Laplace equation in 2D).

We consider a bounded domain $D \subset \mathbb{R}^{2}$ with boundary of class $C^{2}$ and a periodic representation $x:[0,2 \pi] \rightarrow \partial D, t \mapsto\left(x_{1}(t), x_{2}(t)\right)$ of this boundary with $x \in C_{2 \pi}^{2}([0,2 \pi])$. Then, the direct values of the double-layer potential with continuous density $\varphi \in C(\partial D)$ are given by

$$
u(x(t))=\frac{1}{2 \pi} \int_{0}^{2 \pi} \frac{(x(t)-x(\tau)) \cdot \nu(x(\tau))}{|x(t)-x(\tau)|^{2}} \varphi(x(\tau))\left|x^{\prime}(\tau)\right| d \tau
$$

for all $0 \leq t \leq 2 \pi$. If we assume the parametrization to be counterclockwise orientated, then

$$
\nu(x(\tau))=\frac{\left(x_{2}^{\prime}(\tau),-x_{1}^{\prime}(\tau)\right)}{\left|x^{\prime}(\tau)\right|}
$$

defines the exterior unit normal vector at $x(\tau)$ and the integral (4.12) becomes 


$$
u(x(t))=\frac{1}{2 \pi} \int_{0}^{2 \pi} \rho(t, \tau) \varphi(x(\tau)) d \tau, \quad 0 \leq t \leq 2 \pi
$$

with the continuous kernel

$$
\rho(t, \tau)=\left\{\begin{array}{cc}
\frac{\left(x_{1}(t)-x_{1}(\tau)\right) x_{2}^{\prime}(\tau)-\left(x_{2}(t)-x_{2}(\tau)\right) x_{1}^{\prime}(\tau)}{\left(x_{1}(t)-x_{1}(\tau)\right)^{2}+\left(x_{2}(t)-x_{2}(\tau)\right)^{2}}, & t \neq \tau, \\
\frac{1}{2} \frac{x_{2}^{\prime}(t) x_{1}^{\prime \prime}(t)-x_{1}^{\prime}(t) x_{2}^{\prime \prime}(t)}{x_{1}^{\prime}(t)^{2}+x_{2}^{\prime}(t)^{2}} & , \quad t=\tau .
\end{array}\right.
$$

Before we consider the single- and double-layer potentials for the Helmholtz equation, we first have a closer look at the fundamental solution in this case and recall the following asymptotics, see [7].

Theorem 4.4. The fundamental solution

$$
\Phi(x, y)=\frac{i}{4} H_{0}^{(1)}(\kappa|x-y|), \quad x \neq y
$$

of the Helmholtz equation admits the asymptotics

$$
\begin{aligned}
\Phi(x, y)= & \frac{1}{2 \pi} \log \frac{1}{|x-y|}+\frac{i}{4}-\frac{1}{2 \pi}\left(\log \frac{\kappa}{2}+C\right) \\
& +O\left(|x-y|^{2} \log \frac{1}{|x-y|}\right)
\end{aligned}
$$

for $|x-y| \rightarrow 0$, where $C$ denotes the Euler constant $C=0.57721$.

Proof. The Hankel function $H_{0}^{(1)}$ of the first kind and of order zero is defined by

$$
H_{0}^{(1)}=J_{0}+i N_{0}
$$

with the Bessel function $J_{0}$ and the Neumann function $N_{0}$ both of order zero. From the power series expansions

$$
J_{0}(z)=\sum_{k=0}^{\infty} \frac{(-1)^{k}}{(k !)^{2}}\left(\frac{z}{2}\right)^{2 k}
$$

and

$$
N_{0}(z)=\frac{2}{\pi}\left(\log \frac{z}{2}+C\right) J_{0}(z)+\sum_{k=1}^{\infty} a_{k} z^{2 k}
$$

with real coefficients $a_{k}$, see for example [7], we obtain the asymptotics

$$
H_{0}^{(1)}(z)=1+\frac{2 i}{\pi}\left(\log \frac{z}{2}+C\right)+O\left(z^{2} \log \frac{z}{2}\right)
$$

for $z \rightarrow 0$. Substituting $z=\kappa|x-y|$ proves the asymptotics (4.17). 
Example 4.5 (The single-layer potential for the Helmholtz equation in 2D).

We consider a bounded domain $D \subset \mathbb{R}^{2}$ with boundary of class $C^{2}$ and a periodic representation $x:[0,2 \pi] \rightarrow \partial D$ of this boundary with $x \in$ $C_{2 \pi}^{2}([0,2 \pi])$. Then, the direct values of the single-layer potential

$$
u(x(t))=\frac{i}{4} \int_{0}^{2 \pi} H_{0}^{(1)}(\kappa|x(t)-x(\tau)|) \varphi(x(\tau))\left|x^{\prime}(\tau)\right| d \tau, \quad 0 \leq t \leq 2 \pi
$$

can be represented in the form

$$
u(x(t))=\frac{1}{2 \pi} \int_{0}^{2 \pi}\left(\log 4 \sin ^{2} \frac{t-\tau}{2} \rho^{(1)}(t, \tau)+\rho^{(2)}(t, \tau)\right) \varphi(x(\tau)) d \tau
$$

for all $0 \leq t \leq 2 \pi$ with the continuous functions

$$
\rho^{(1)}(t, \tau)=-\frac{\left|x^{\prime}(\tau)\right|}{2} J_{0}(\kappa|x(t)-x(\tau)|)
$$

and

$$
\rho^{(2)}(t, \tau)=\frac{i \pi}{2}\left|x^{\prime}(\tau)\right| H_{0}^{(1)}(\kappa|x(t)-x(\tau)|)-\log 4 \sin ^{2} \frac{t-\tau}{2} \rho^{(1)}(t, \tau)
$$

for $(t, \tau) \in[0,2 \pi]^{2}$. The diagonal terms $\rho^{(2)}(t, t)$ are given by

$$
\rho^{(2)}(t, t)=\left|x^{\prime}(t)\right|\left(\frac{\pi i}{2}-\log \frac{\kappa}{2}-C-\log \left|x^{\prime}(t)\right|\right), \quad 0 \leq t \leq 2 \pi,
$$

which can be deduced by inserting the asymptotics (4.17) into (4.25) and using (4.11).

Finally, we present a splitting for the double-layer potential for Helmholtz' equation for which we have to calculate the first derivative of the Hankel function. Thus we recall the following recursion formulae for the Hankel functions.

Lemma 4.6. The derivatives of the Hankel functions $H_{\nu}^{(1)}=J_{\nu}+i N_{\nu}$ of the first kind satisfy the relations

$$
-2 \frac{d H_{\nu}^{(1)}}{d z}=H_{\nu+1}^{(1)}-H_{\nu-1}^{(1)}
$$

and

$$
H_{\nu-1}^{(1)}(z)+H_{\nu+1}^{(1)}(z)=\frac{2 \nu}{z} H_{\nu}^{(1)}(z)
$$

for all $\nu \in \mathbb{R}$ and $z \in \mathbb{R} \backslash\{0\}$, where the parameter $\nu$ indicates the order of the Hankel, Bessel and Neumann function. In particular, there holds

$$
\frac{d H_{0}^{(1)}}{d z}=-H_{1}^{(1)}=-J_{1}-i N_{1}
$$


For the proof of this Lemma we refer the reader to [72]. We are now prepared to handle the double-layer potential in the case of Helmholtz' equation.

Example 4.7 (The double-layer potential for the Helmholtz equation in 2D).

We consider a bounded domain $D \subset \mathbb{R}^{2}$ with boundary of class $C^{2}$ and a periodic representation $x:[0,2 \pi] \rightarrow \partial D, t \mapsto\left(x_{1}(t), x_{2}(t)\right)$ of this boundary with $x \in C_{2 \pi}^{2}([0,2 \pi])$. Then the direct values of the double-layer potential with continuous density $\varphi \in C(\partial D)$ are given by

$u(x(t))=\frac{i \kappa}{4} \int_{0}^{2 \pi} H_{1}^{(1)}(\kappa|x(t)-x(\tau)|) \frac{(x(t)-x(\tau)) \cdot \nu(x(\tau))}{|x(t)-x(\tau)|} \varphi(x(\tau))\left|x^{\prime}(\tau)\right| d \tau$

for all $0 \leq t \leq 2 \pi$. Again, we assume the parametrization to be counterclockwise orientated. Thus we can split the above integral into (4.23) as before with the continuous functions

$$
\rho^{(1)}(t, \tau)=-\frac{\kappa}{2} \frac{(x(t)-x(\tau)) \cdot \nu(x(\tau))}{|x(t)-x(\tau)|} J_{1}(\kappa|x(t)-x(\tau)|)\left|x^{\prime}(\tau)\right|
$$

and

$$
\begin{aligned}
\rho^{(2)}(t, \tau)= & \frac{i \kappa \pi}{2} H_{1}^{(1)}(\kappa|x(t)-x(\tau)|) \frac{(x(t)-x(\tau)) \cdot \nu(x(\tau))}{|x(t)-x(\tau)|}\left|x^{\prime}(\tau)\right| \\
& -\log 4 \sin ^{2} \frac{t-\tau}{2} \rho^{(1)}(t, \tau) .
\end{aligned}
$$

The second term in the last equation vanishes, when $t \rightarrow \tau$. Furthermore, from the asymptotics (4.21) for the Hankel function $H_{0}^{(1)}$ we obtain

$$
\lim _{z \rightarrow 0} z H_{1}^{(1)}(z)=-\lim _{z \rightarrow 0} z \frac{d}{d z} H_{0}^{(1)}(z)=\frac{2}{\pi i} .
$$

Hence, together with (4.15) this yields the diagonal terms

$$
\rho^{(2)}(t, t)=\frac{1}{2} \frac{x_{2}^{\prime}(t) x_{1}^{\prime \prime}(t)-x_{1}^{\prime}(t) x_{2}^{\prime \prime}(t)}{x_{1}^{\prime}(t)^{2}+x_{2}^{\prime}(t)^{2}}
$$

of the function $\rho^{(2)}$.

\subsection{A Dirichlet Boundary Value Problem}

In the following we will apply the integral equation method to solve two Dirichlet boundary value problems. While the first boundary value problem for the Helmholtz equation arises from acoustics, the second boundary value problem deals with Laplace's equation and originates from the field of electrostatics. Based on Nyström's method and a separation of the logarithmic singularity of the layer potentials we present a numerical solution 
to these boundary value problems and show some numerical examples. The numerical scheme under consideration will be used in the sequel to generate simulated data for the reconstruction algorithms.

Let us first consider Helmholtz' equation, which describes the propagation of sound waves in a homogeneous medium. If we locate a sound-soft scatterer $D \subset \mathbb{R}^{m}$ in this medium, any incoming sound wave is scattered by the obstacle in such a way that the sum of the incident wave $u^{i}$ and the scattered wave $u^{s}$ vanishes on the boundary $\partial D$ of the scatterer. The total field $u=u^{i}+u^{s}$ in the domain $\Omega \backslash \bar{D}$ is then given as the solution to the Helmholtz equation

$$
\Delta u+\kappa^{2} u=0
$$

in $\Omega \backslash \bar{D}$ with the Dirichlet boundary conditions

$$
\left.u\right|_{\partial D}=0,\left.\quad u\right|_{\partial \Omega}=f
$$

for a given continuous function $f$ and a complex parameter $\kappa$ with

$$
\operatorname{Re}(\kappa)>0, \quad \operatorname{Im}(\kappa) \geq 0 .
$$

We refer to the problem of finding a solution to (4.35) - (4.36) as the direct boundary value problem.

For arbitrary domains we can not expect uniqueness of this problem unless $-\kappa^{2}$ is not a Dirichlet eigenvalue of the operator $\Delta$ in the domain $\Omega \backslash \bar{D}$. But if we forbid these special values for $\kappa$ we can prove the following.

Theorem 4.8. Let $D, \Omega \subset \mathbb{R}^{m}, m=2,3$ be bounded domains with $D \subset \Omega$ and boundaries $\partial D, \partial \Omega$ of class $C^{2}$. Then the problem (4.35) - (4.36) has a unique solution in $C^{2}(\Omega \backslash \bar{D}) \cap C(\bar{\Omega} \backslash D)$, provided $-\kappa^{2}$ is not an eigenvalue of the Laplace operator in $\Omega \backslash \bar{D}$.

Proof. The uniqueness of this problem is assured by the assumption on $\kappa^{2}$. We only have to show the existence of a solution. We do this by seeking a solution of (4.35) in the form of a combined layer

$$
\begin{aligned}
u(x) & =\int_{\partial D}\left\{\frac{\partial \Phi(x, y)}{\partial \nu(y)}-i \eta \Phi(x, y)\right\} \varphi_{1}(y) d s(y) \\
& +\int_{\partial \Omega} \frac{\partial \Phi(x, y)}{\partial \nu(y)} \varphi_{2}(y) d s(y)
\end{aligned}
$$

in $\Omega \backslash \bar{D}$ with continuous densities $\varphi_{1} \in C(\partial D), \varphi_{2} \in C(\partial \Omega)$ and a real coupling parameter $\eta>0$. Here $\nu(y)$ denotes the unit normal vector to $y \in \partial(\Omega \backslash \bar{D})$ pointing into the exterior of $\Omega \backslash \bar{D}$. Clearly $u$ is a solution to the Helmholtz equation in $\Omega \backslash \bar{D}$. In order to solve the problem $(4.35)-(4.36)$ the function $u$ has to admit the boundary values $u=0$ on $\partial D$ and $u=f$ on $\partial \Omega$. Since $\partial D \cap \partial \Omega=\emptyset$ we define $\Gamma_{1}:=\partial D, \Gamma_{2}:=\partial \Omega$ and recall the 
notation $S_{j k}$ and $K_{j k}$ for the boundary integral operators, see (3.33). These integral operators correspond to two times the direct values of the singleand double-layer potential with integration domain $\Gamma_{k}$ evaluated on the domain $\Gamma_{j}$ for $1 \leq j, k \leq 2$. With the jump relations (3.27) for the doublelayer potential the boundary conditions (4.36) for $u$ turn into a system of two operator equations

$$
\begin{aligned}
& 0=K_{11} \varphi_{1}-\varphi_{1}-i \eta S_{11} \varphi_{1}+K_{12} \varphi_{2} \quad \text { on } \Gamma_{1} \text {, } \\
& 2 f=K_{21} \varphi_{1}-i \eta S_{21} \varphi_{1}+K_{22} \varphi_{2}-\varphi_{2} \quad \text { on } \Gamma_{2} \text {. }
\end{aligned}
$$

For $j=k$ the operators $S_{j k}$ and $K_{j k}$ are compact due to Theorem 3.13, otherwise the compactness follows with Theorem 3.2 since the integral operators have a continuous kernel in $C\left(\Gamma_{j} \times \Gamma_{k}\right)$ for $j \neq k$. We introduce the notation $X$ for the product space $C\left(\Gamma_{1}\right) \times C\left(\Gamma_{2}\right)$ and $I$ for the identity operator in this space. Then we can summarize (4.39) and (4.40) in the following operator equation of the second kind

$$
(I-L) \psi=g
$$

with the compact operator $L: X \rightarrow X$ defined by

$$
L=\left(\begin{array}{ll}
K_{11}-i \eta S_{11} & K_{12} \\
K_{21}-i \eta S_{21} & K_{22}
\end{array}\right)
$$

the density

$$
\psi=\left(\begin{array}{c}
\varphi_{1} \\
\varphi_{2}
\end{array}\right) \in X
$$

and the right hand side

$$
g=\left(\begin{array}{c}
0 \\
-2 f
\end{array}\right) \in X
$$

We prove the injectivity of the operator $I-L$. Let $\psi$ be a solution of the homogeneous equation $(I-L) \psi=0$, then the combined layer $u$ with density $\psi$ is a solution of the Helmholtz equation in $\Omega \backslash \bar{D}$ with vanishing Dirichlet boundary values. By the assumption on $\kappa^{2}$, i.e. the uniqueness of the boundary value problem, this solution can only be $u \equiv 0$. In particular the boundary data $u_{-}$and $\frac{\partial u_{-}}{\partial \nu}$ vanish on $\Gamma_{1} \cup \Gamma_{2}$. With the behaviour of the layer potentials expressed in Theorem 3.11 we conclude

$$
\frac{\partial u_{+}}{\partial \nu}=\frac{\partial u_{+}}{\partial \nu}-\frac{\partial u_{-}}{\partial \nu}=i \eta \varphi_{1}
$$

and

$$
u_{+}=u_{+}-u_{-}=\varphi_{1}
$$

for the limiting values of $u$ and its normal derivative on $\Gamma_{1}$. We can now apply Green's theorem in the domain $D$ to obtain 


$$
\int_{D}|\nabla u|^{2}-\kappa^{2}|u|^{2} d x=\int_{\Gamma_{1}} \bar{u}_{+} \frac{\partial u_{+}}{\partial \nu} d s=i \eta \int_{\Gamma_{1}}\left|\varphi_{1}\right|^{2} d s .
$$

Taking the imaginary part on both sides and recalling the assumptions (4.37) on $\kappa$ yields

$$
0 \geq-\operatorname{Im} \kappa^{2} \int_{D}|u|^{2} d x=\eta \int_{\Gamma_{1}}\left|\varphi_{1}\right|^{2} d s \geq 0
$$

which implies $\varphi_{1}=0$.

On the second boundary $\Gamma_{2}$ we obtain

$$
\frac{\partial u_{+}}{\partial \nu}=\frac{\partial u_{+}}{\partial \nu}-\frac{\partial u_{-}}{\partial \nu}=0
$$

from (3.28). Thus $u$ solves the exterior Neumann problem with vanishing boundary data on $\Gamma_{2}$, which yields $u \equiv 0$ in $\mathbb{R}^{m} \backslash \bar{\Omega}$ by Theorem 2.20 . Now we also derive

$$
\varphi_{2}=u_{+}-u_{-}=0
$$

on $\Gamma_{2}$ from the jump relation (3.27).

Therefore the operator $I-L$ is injective and Riesz' Theorem 3.14 yields the bijectivity of this operator and the existence of a bounded linear inverse, which proves the existence of a solution to problem $(4.35)-(4.36)$.

The previous proof provides a constructive solution of the direct boundary value problem for the Helmholtz equation in form of a combined layer. We mention that one can modify the solution theory for the boundary value problem by omitting the terms involving the boundary $\partial \Omega$ and deduce a solution theory for the direct sound-soft scattering problem. In the following we describe the numerical implementation of the demonstrated solution theory with Nyström's method. We restrict ourselves to the boundary value problem and present a numerical scheme exemplarily in the two-dimensional case.

The unique solution to the direct boundary value problem $(4.35)-(4.36)$ is given by the combined layer potential (4.38) where the density $\psi \in C\left(\Gamma_{1}\right) \times$ $C\left(\Gamma_{2}\right)$ is the unique solution to the integral equation (4.41). For numerical accuracy we separate the logarithmic singularity of the single-layer potential as described in Example 4.5 and also separate the kernel of the double-layer potential as shown in Example 4.7 to deal with the logarithmic singularities occuring in its derivatives. Then we solve (4.41) with Nyström's method for integral operators with weakly singular kernels.

In three dimensions the separation of the singularity is more complicated and we refer to [77] for an analytical treatment of the weakly-singular kernels of the layer potentials in this case. Focusing on the inverse problems and to simplify matters we will use a quadrature rule which ignores the singularities 
of the integrands instead. We will come back to this topic in the second part of this work, when we show numerical examples for the reconstruction of three-dimensional sound-soft obstacles in inverse acoustic scattering.

We first introduce a parametrization

$$
x_{1}:[0,2 \pi] \rightarrow \Gamma_{1}, \quad t \mapsto x_{1}(t)=\left(x_{1,1}(t), x_{1,2}(t)\right)
$$

of the boundary $\Gamma_{1}$ and

$$
x_{2}:[0,2 \pi] \rightarrow \Gamma_{2}, \quad t \mapsto x_{2}(t)=\left(x_{2,1}(t), x_{2,2}(t)\right)
$$

of the boundary $\Gamma_{2}$. We will demonstrate the order of convergence numerically for analytic boundaries, i.e. we assume the functions $x_{1}$ and $x_{2}$ to be analytic. To simplify notations we write

$$
\tilde{\chi}_{j}(t):=\chi_{j}\left(x_{j}(t)\right), \quad t \in[0,2 \pi]
$$

for functions $\chi_{j}$ in $C\left(\Gamma_{j}\right), j=1,2$, and

$$
\tilde{\Psi}(t):=\left(\begin{array}{c}
\tilde{\chi}_{1}(t) \\
\tilde{\chi}_{2}(t)
\end{array}\right), \quad t \in[0,2 \pi]
$$

for elements $\Psi=\left(\chi_{1}, \chi_{2}\right)^{t}$ of the product space $X$. Now, we can substitute the integral equation (4.41) by its parametric form

$$
(I-A) \tilde{\psi}=\tilde{g}
$$

with the operator $A: C([0,2 \pi]) \rightarrow C([0,2 \pi])$ of the form

$$
A=\left(\begin{array}{ll}
A_{11} & A_{12} \\
A_{21} & A_{22}
\end{array}\right) \text {. }
$$

More precisely, the diagonal operators of $A$ are given by

$$
\begin{aligned}
\left(A_{j j} \tilde{\varphi}_{j}\right)(t)= & \frac{1}{2 \pi}\left\{\int_{0}^{2 \pi} \log 4 \sin ^{2} \frac{t-\tau}{2} \rho_{j}^{(1)}(t, \tau) \tilde{\varphi}_{j}(\tau) d \tau\right. \\
& \left.+\int_{0}^{2 \pi} \rho_{j}^{(2)}(t, \tau) \tilde{\varphi}_{j}(\tau) d \tau\right\}
\end{aligned}
$$

with

$$
\begin{aligned}
\rho_{j}^{(1)}(t, \tau)= & -\kappa \frac{\left(x_{j}(t)-x_{j}(\tau)\right) \cdot \tilde{\nu}_{j}(\tau)}{\left|x_{j}(t)-x_{j}(\tau)\right|} J_{1}\left(\kappa\left|x_{j}(t)-x_{j}(\tau)\right|\right)\left|x_{j}{ }^{\prime}(\tau)\right| \\
& +\delta_{1 j} i \eta\left|x_{j}{ }^{\prime}(\tau)\right| J_{0}\left(\kappa\left|x_{j}(t)-x_{j}(\tau)\right|\right) \\
\rho_{j}^{(2)}(t, \tau)= & i \kappa \pi H_{1}^{(1)}\left(\kappa\left|x_{j}(t)-x_{j}(\tau)\right|\right) \frac{\left(x_{j}(t)-x_{j}(\tau)\right) \cdot \tilde{\nu}_{j}(\tau)}{\left|x_{j}(t)-x_{j}(\tau)\right|}\left|x_{j}{ }^{\prime}(\tau)\right| \\
& +\delta_{1 j} \eta \pi\left|x_{j}{ }^{\prime}(\tau)\right| H_{0}^{(1)}\left(\kappa\left|x_{j}(t)-x_{j}(\tau)\right|\right) \\
& -\log 4 \sin ^{2} \frac{t-\tau}{2} \rho_{j}^{(1)}(t, \tau)
\end{aligned}
$$


for $j=1,2$ and $t, \tau \in[0,2 \pi]$ with $t \neq \tau$. We deduce the diagonal elements of the kernels $\rho_{j}^{(k)}$ from Example 4.5 and Example 4.7 and obtain

$$
\rho_{j}^{(1)}(t, t)=\delta_{1 j} i \eta\left|x_{j}{ }^{\prime}(t)\right|
$$

and

$$
\begin{aligned}
\rho_{j}^{(2)}(t, t)= & \frac{x_{j, 2}{ }^{\prime}(t) x_{j, 1}{ }^{\prime \prime}(t)-x_{j, 1}{ }^{\prime}(t) x_{j, 2}{ }^{\prime \prime}(t)}{\left(x_{j, 1}{ }^{\prime}(t)\right)^{2}+\left(x_{j, 2}{ }^{\prime}(t)\right)^{2}} \\
& -2 \delta_{1 j} i \eta\left|x_{j}{ }^{\prime}(t)\right|\left(\frac{\pi i}{2}-\log \frac{\kappa}{2}-C-\log \left|x_{j}{ }^{\prime}(t)\right|\right) .
\end{aligned}
$$

Furthermore the off-diagonal operators are defined by

$$
\left(A_{j k} \tilde{\varphi}_{k}\right)(t)=\frac{1}{2 \pi} \int_{0}^{2 \pi} \sigma_{j k}(t, \tau) \tilde{\varphi}_{k}(\tau) d \tau
$$

for $j, k=1,2, j \neq k$ with the kernels

$$
\begin{aligned}
\sigma_{j k}(t, \tau)= & i \kappa \pi H_{1}^{(1)}\left(\kappa\left|x_{j}(t)-x_{k}(\tau)\right|\right) \frac{\left(x_{j}(t)-x_{k}(\tau)\right) \cdot \tilde{\nu}_{k}(\tau)}{\left|x_{j}(t)-x_{k}(\tau)\right|}\left|x_{k}{ }^{\prime}(\tau)\right| \\
& +\delta_{1 k} \eta \pi\left|x_{k}{ }^{\prime}(\tau)\right| H_{0}^{(1)}\left(\kappa\left|x_{j}(t)-x_{k}(\tau)\right|\right)
\end{aligned}
$$

for $t, \tau \in[0,2 \pi]$.

For the approximation of the integral operator $A$ with numerical integration operators we will apply the trapezoidal rule (3.59) to the analytic part and the quadrature formula (4.5) to the logarithmic part of the kernel. To this end we divide the interval $[0,2 \pi]$ into the $2 n_{1}$ equally distributed points

$$
t_{1, k}=\frac{\pi k}{n_{1}}, \quad k=0, \ldots, 2 n_{1}-1
$$

for the numerical integration over the boundary $\Gamma_{1}$ and into the $2 n_{2}$ equally distributed points

$$
t_{2, k}=\frac{\pi k}{n_{2}}, \quad k=0, \ldots, 2 n_{2}-1
$$

for the numerical integration over the boundary $\Gamma_{2}$. Then we can approximate the integral operator $A$ with a numerical integration operator $\tilde{A}$ of the form

$$
\tilde{A}=\left(\begin{array}{ll}
\tilde{A}_{11} & \tilde{A}_{12} \\
\tilde{A}_{21} & \tilde{A}_{22}
\end{array}\right)
$$

where the entries of this operator matrix are defined by

$$
\left(\tilde{A}_{j j} \tilde{\varphi}_{j}\right)(t)=\sum_{l=0}^{2 n_{j}-1}\left(R_{l}^{\left(n_{j}\right)}(t) \rho_{j}^{(1)}\left(t, t_{j, l}\right)+\frac{1}{2 n_{j}} \rho_{j}^{(2)}\left(t, t_{j, l}\right)\right) \tilde{\varphi}_{j}\left(t_{j, l}\right)
$$


for $j=1,2$ and

$$
\left(\tilde{A}_{j k} \tilde{\varphi}_{k}\right)(t)=\sum_{l=0}^{2 n_{k}-1} \frac{1}{2 n_{k}} \sigma_{j k}\left(t, t_{k, l}\right) \tilde{\varphi}_{k}\left(t_{k, l}\right)
$$

for $j, k=1,2, j \neq k$ and $t \in[0,2 \pi]$. The resulting semi-discrete equation

$$
(I-\tilde{A}) \tilde{\psi}=\tilde{g}
$$

can be solved with the Nyström method. Hence a solution $\tilde{\psi}=\left(\tilde{\psi}_{1}, \tilde{\psi}_{2}\right)^{t}$ of (4.69) is given by

$$
\begin{aligned}
\tilde{\psi}_{1}(t):= & \sum_{l=0}^{2 n_{1}-1}\left(R_{l}^{\left(n_{1}\right)}(t) \rho_{1}^{(1)}\left(t, t_{1, l}\right)+\frac{1}{2 n_{1}} \rho_{1}^{(2)}\left(t, t_{1, l}\right)\right) \hat{\psi}_{1, l} \\
& +\sum_{l=0}^{2 n_{2}-1} \frac{1}{2 n_{2}} \sigma_{12}\left(t, t_{2, l}\right) \hat{\psi}_{2, l}, \\
\tilde{\psi}_{2}(t):= & -2 \tilde{f}(t)+\sum_{l=0}^{2 n_{1}-1} \frac{1}{2 n_{1}} \sigma_{21}\left(t, t_{1, l}\right) \hat{\psi}_{1, l} \\
& +\sum_{l=0}^{2 n_{2}-1}\left(R_{l}^{\left(n_{2}\right)}(t) \rho_{2}^{(1)}\left(t, t_{2, l}\right)+\frac{1}{2 n_{2}} \rho_{2}^{(2)}\left(t, t_{2, l}\right)\right) \hat{\psi}_{2, l},
\end{aligned}
$$

when $\hat{\psi}=\left(\hat{\psi}_{1,0}, \ldots, \hat{\psi}_{1,2 n_{1}-1}, \hat{\psi}_{2,0}, \ldots, \hat{\psi}_{2,2 n_{2}-1}\right)^{t}$ solves the linear system

$$
\hat{A} \hat{\psi}=\hat{g} .
$$

Here the the block matrix

$$
\hat{A}=\left(\begin{array}{ll}
\hat{A}_{11} & \hat{A}_{12} \\
\hat{A}_{21} & \hat{A}_{22}
\end{array}\right)
$$

is defined via its submatrices

$$
\hat{A}_{j j}^{(m, l)}=R_{l}^{\left(n_{j}\right)}\left(t_{j, m}\right) \rho_{j}^{(1)}\left(t_{j, m}, t_{j, l}\right)+\frac{1}{2 n_{j}} \rho_{j}^{(2)}\left(t_{j, m}, t_{j, l}\right)
$$

for $j=1,2$ and $l, m=0, \ldots, 2 n_{j}-1$, and

$$
\hat{A}_{j k}^{(m, l)}=\frac{1}{2 n_{k}} \sigma_{j k}\left(t_{j, m}, t_{k, l}\right)
$$

for $j, k=1,2, j \neq k$ and $l=0, \ldots, 2 n_{k}-1, m=0, \ldots, 2 n_{j}-1$. Furthermore, the vector $\hat{g} \in \mathbb{C}^{2\left(n_{1}+n_{2}\right)}$ is given by

$$
\hat{g}=-2\left(0, \ldots, 0, \tilde{f}\left(t_{2,0}\right), \ldots, \tilde{f}\left(t_{2,2 n_{2}-1}\right)\right)^{t} .
$$


We present some numerical examples in the following three settings, see Figure 4.1. In all of these settings the domain $\Omega$ of interest is the unit circle. In Setting 1 we choose the obstacle $D$ in form of an ellipse whose boundary is paramterized by

$$
\partial D=\{(0.2 \cos t, 0.3 \sin t): 0 \leq t \leq 2 \pi\} .
$$

The kite-shaped domain $D$ in the second setting admits the boundary representation

$$
\partial D=\{0.2 \cdot(-\cos t-0.65 \cos 2 t+0.65,1.5 \sin t): 0 \leq t \leq 2 \pi\}
$$

and the obstacle in Setting 3 is given by

$\partial D=\{0.25 \cdot(0.5 \cos (2 \pi-t)+0.08 \sin (4 \cdot(2 \pi-t)), 1.5 \sin (2 \pi-t)): 0 \leq t \leq 2 \pi\}$.

Then we solve the direct boundary value problem

$$
\Delta u+\kappa^{2} u=0 \quad \text { in } \Omega \backslash \bar{D},
$$

where the boundary data on $\partial \Omega$ and $\partial D$ is given by the restriction of

$$
u_{\text {true }}(x)=\Phi\left(x, z_{i}\right)+\Phi\left(x, z_{e}\right), \quad x \in \bar{\Omega} \backslash D
$$

to the boundary $\partial \Omega$ and $\partial D$ respectively. Here both source points $z_{i}=(0,0)$ and $z_{e}=(1.5,0)$ lie in the exterior of the domain $\Omega \backslash \bar{D}$ and therefore $u$ solves the Helmholtz equation in $\Omega \backslash \bar{D}$. We compare the true solution $u_{\text {true }}$ with the solution $u_{\text {calc }}$ calculated with the presented numerical algorithm in the arbitrarily chosen point $z=(0.5,0.5) \in \Omega \backslash \bar{D}$. The modulus of the error is illustrated in Tables 4.1-4.3, in which the exponential convergence of the algorithm is clearly exhibited.

As a second problem we consider a special situation in electric impedance tomography, where we search the electric field of a perfectly conducting inclusion in a homogeneous background medium. The corresponding boundary value problem can be formulated as

$$
\begin{aligned}
\Delta u=0 & \text { in } \Omega \backslash \bar{D}, \\
u=0 & \text { on } \partial D, \\
u=f & \text { on } \partial \Omega
\end{aligned}
$$

for a continuous function $f \in C(\partial \Omega)$. In the case of electric impedance tomography, the combined layer approach (4.38) does not lead to a solution of the boundary value problem and needs to be modified. Following the ideas presented in [36] we use a modified potential approach and simplify the existence proof given in [14] which was based on the method of Mikhlin [45]. 
Theorem 4.9. Let $D, \Omega \subset \mathbb{R}^{m}, m=2,3$ be two bounded domains with boundary of class $C^{2}$ such that $D \subset \Omega$. Then the problem (4.83) - (4.85) possesses a unique solution in $C^{2}(\Omega \backslash \bar{D}) \cap C(\bar{\Omega} \backslash D)$.

Proof. Obviously, by the maximum-principle for harmonic functions there can be at most one solution to the problem (4.83) - (4.85).

We recall the notation $\Gamma_{1}=\partial D$ and $\Gamma_{2}=\partial \Omega$ for the disjoint boundaries of the domain $\Omega \backslash \bar{D}$. Then, the modified double layer ansatz

$$
u(x)=\int_{\Gamma_{1}}\left(\frac{\partial \Phi(x, y)}{\partial \nu(y)}+\log \frac{1}{|x|}\right) \varphi_{1}(y) d s(y)+\int_{\Gamma_{2}} \frac{\partial \Phi(x, y)}{\partial \nu(y)} \varphi_{2}(y) d s(y)
$$

for $m=2$ and

$$
u(x)=\int_{\Gamma_{1}}\left(\frac{\partial \Phi(x, y)}{\partial \nu(y)}+\frac{1}{|x|}\right) \varphi_{1}(y) d s(y)+\int_{\Gamma_{2}} \frac{\partial \Phi(x, y)}{\partial \nu(y)} \varphi_{2}(y) d s(y)
$$

for $m=3$ with continuous densities $\varphi_{1} \in C\left(\Gamma_{1}\right), \varphi_{2} \in C\left(\Gamma_{2}\right)$ is defined for $x \in \mathbb{R}^{m} \backslash\left(\Gamma_{1} \cup \Gamma_{2} \cup\{0\}\right)$ and solves Laplace's equation in $\Omega \backslash \bar{D}$. Here we assume without any loss of generality that $0 \in D$, otherwise we substitute $1 /|x|$ in the additional terms of the modified double layer ansatz by $1 /\left|x-x_{0}\right|$ with some $x_{0} \in D$. Furthermore the normal vector $\nu(y)$ is assumed to be orientated into the exterior of the domain $\Omega \backslash \bar{D}$ for all $y \in \Gamma_{1} \cup \Gamma_{2}$.

In order to fulfill the prescribed boundary values, the unknown continuous densities $\varphi_{1}$ and $\varphi_{2}$ have to solve the system of integral equations

$$
\begin{aligned}
& 0=K_{11} \varphi_{1}-\varphi_{1}+M_{11} \varphi_{1}+K_{12} \varphi_{2} \quad \text { on } \Gamma_{1}, \\
& 2 f=K_{21} \varphi_{1}+M_{21} \varphi_{1}+K_{22} \varphi_{2}-\varphi_{2} \quad \text { on } \Gamma_{2} \text {, }
\end{aligned}
$$

where the modification operators $M_{j 1}: \Gamma_{1} \rightarrow \Gamma_{j}$ are defined by

$$
\left(M_{j 1} \varphi_{1}\right)(x):=\left\{\begin{array}{cc}
2 \log \frac{1}{|x|} \int_{\Gamma_{1}} \varphi_{1}(y) d s(y), & m=2, \\
\frac{2}{|x|} \int_{\Gamma_{1}} \varphi_{1}(y) d s(y), & m=3,
\end{array}\right.
$$

for $x \in \Gamma_{j}$ and $j=1,2$. Since the kernels of the modification operators $M_{j 1}$, $j=1,2$, are continuous the integral operators $M_{j 1}$ are compact. Thus we obtain again an integral equation of the second kind, which can be written in the form (4.41) on the product space $C\left(\Gamma_{1}\right) \times C\left(\Gamma_{2}\right)$, where we have to replace the operator matrix $(4.42)$ by

$$
L=\left(\begin{array}{ll}
K_{11}+M_{11} & K_{12} \\
K_{21}+M_{21} & K_{22}
\end{array}\right) .
$$

It is sufficient to verify the injectivity of the homogeneous equation which is equivalent to the existence of a bounded inverse operator, i.e. the stable 
and unique solvability of the above system of integral equations, due to the theorem of Riesz.

Let $\varphi_{1} \in C\left(\Gamma_{1}\right)$ and $\varphi_{2} \in C\left(\Gamma_{2}\right)$ be two continuous densities such that they solve equations (4.88) and (4.89) with a zero right-hand side. Then $u$ is a solution to the Laplace equation in $\Omega \backslash \bar{D}$ with homogeneous boundary data and by the uniqueness of this problem $u \equiv 0$ in $\Omega \backslash \bar{D}$. Hence, Theorem 3.11 implies

$$
\frac{\partial u_{+}}{\partial \nu}=\frac{\partial u_{-}}{\partial \nu}=0
$$

on $\Gamma_{1} \cup \Gamma_{2}$. We choose an $\varepsilon>0$ such that the ball $B_{\varepsilon}(0)$ is contained in $D$. Since $u$ is harmonic in $D \backslash \overline{B_{\varepsilon}(0)}$ we deduce

$$
\int_{\partial B_{\varepsilon}(0)} \frac{\partial u}{\partial \nu} d s=\int_{\Gamma_{1}} \frac{\partial u}{\partial \nu} d s+\int_{\partial B_{\varepsilon}(0)} \frac{\partial u}{\partial \nu} d s=0
$$

from Green's second theorem applied to the functions $u$ and $v=1$. With the same argumentation we obtain

$$
\int_{\partial B_{\varepsilon}(0)} \frac{\partial v_{1}}{\partial \nu} d s+\int_{\partial B_{\varepsilon}(0)} \frac{\partial v_{2}}{\partial \nu} d s=0
$$

for the functions

$$
v_{1}(x)=\int_{\Gamma_{1}} \frac{\partial \Phi(x, y)}{\partial \nu(y)} \varphi_{1}(y) d s(y)
$$

and

$$
v_{2}(x)=\int_{\Gamma_{2}} \frac{\partial \Phi(x, y)}{\partial \nu(y)} \varphi_{2}(y) d s(y),
$$

which are harmonic in the ball $B_{\varepsilon}(0)$. Inserting the definition (4.86), (4.87) of $u$ in (4.93) and using (4.94) yields

$$
\begin{aligned}
\int_{\partial B_{\varepsilon}(0)} \frac{\partial u}{\partial \nu} d s & =\int_{\partial B_{\varepsilon}(0)} \frac{\partial}{\partial \nu(x)} \log \frac{1}{|x|} \int_{\Gamma_{1}} \varphi_{1}(y) d s(y) d s(x) \\
& =-2 \pi \int_{\Gamma_{1}} \varphi_{1}(y) d s(y)
\end{aligned}
$$

for $m=2$ and

$$
\begin{aligned}
\int_{\partial B_{\varepsilon}(0)} \frac{\partial u}{\partial \nu} d s & =\int_{\partial B_{\varepsilon}(0)} \frac{\partial}{\partial \nu(x)} \frac{1}{|x|} \int_{\Gamma_{1}} \varphi_{1}(y) d s(y) d s(x) n \\
& =-4 \pi \int_{\Gamma_{1}} \varphi_{1}(y) d s(y)
\end{aligned}
$$

for $m=3$. In either case (4.93) implies

$$
\int_{\Gamma_{1}} \varphi_{1}(y) d s(y)=0
$$


which shows that the function $u$ admits the representation $u=v_{1}+v_{2}$ and turns out to be harmonic in all of $D$. Moreover, $u$ is a solution to the interior Neumann problem with homogeneous boundary data in $D$ and we obtain $u \equiv c$ from Theorem 2.15. The jump relations (3.27) on $\Gamma_{1}$ yield

$$
\varphi_{1}=u_{+}-u_{-}=u_{+}=c,
$$

and together with (4.99) this proves that $\varphi_{1}=0$. Finally, from the uniqueness of the exterior Neumann problem it follows that $u$ vanishes in all of $\mathbb{R}^{m} \backslash \bar{\Omega}$. Hence the jump relations $(3.27)$ on $\Gamma_{2}$ prove $\varphi_{2}=0$, i.e. the injectivity of the homogeneous system of integral equations $(4.88)-(4.89)$. 

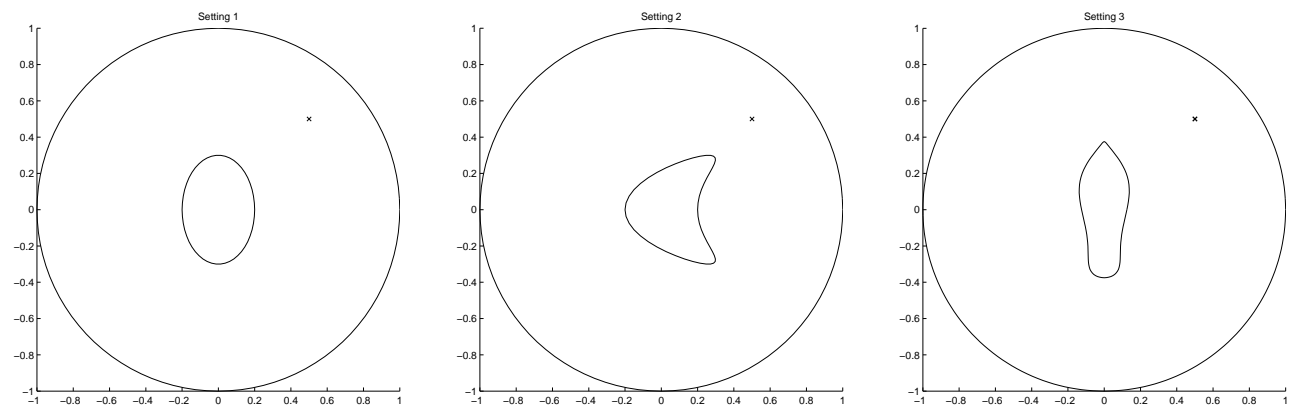

Fig. 4.1. Setting 1 to Setting 3 indicate the boundary of the domain $\partial \Omega$ and $\partial D$ respectively. In addition, we have marked the point $(0.5,0.5)$ in which we evaluate both the true and calculated solution.

\begin{tabular}{ccccc}
\hline \hline$n$ & $\kappa=0.5$ & $\kappa=1$ & $\kappa=2$ & $\kappa=5$ \\
\hline 4 & 0.03729685 & 0.02745902 & 0.01859241 & 0.01684003 \\
8 & 0.00100308 & 0.00099435 & 0.00108266 & 0.00214399 \\
16 & 0.00000489 & 0.00000436 & 0.00000406 & 0.00000708 \\
32 & 0.00000001 & 0.00000001 & 0.00000001 & 0.00000003 \\
\hline \hline
\end{tabular}

Table 4.1. The Table shows the error $\left|u_{\text {calc }}-u_{\text {true }}\right|$ for the ellipsoidal obstacle for several wave numbers and an increasing number of sampling points on the boundaries. Here $n$ denotes the number of sampling points on $\partial D$, i.e. $n=2 n_{1}$. Furthermore, $n_{2}$ is chosen such that $n_{2}=2 n_{1}$.

\begin{tabular}{ccccc}
\hline \hline$n$ & $\kappa=0.5$ & $\kappa=1$ & $\kappa=2$ & $\kappa=5$ \\
\hline 4 & 0.03132068 & 0.02596965 & 0.01909369 & 0.15177646 \\
8 & 0.00203505 & 0.00153830 & 0.00090788 & 0.00720775 \\
16 & 0.00078572 & 0.00040440 & 0.00022458 & 0.00047274 \\
32 & 0.00000222 & 0.00000114 & 0.00000064 & 0.00000138 \\
\hline \hline
\end{tabular}

Table 4.2. The Table shows the error $\left|u_{\text {calc }}-u_{\text {true }}\right|$ for the kite-shaped obstacle for several wave numbers and an increasing number of sampling points on the boundaries. Here $n$ denotes the number of sampling points on $\partial D$, i.e. $n=2 n_{1}$. Furthermore, $n_{2}$ is chosen such that $n_{2}=2 n_{1}$.

\begin{tabular}{ccccc}
\hline \hline$n$ & $\kappa=0.5$ & $\kappa=1$ & $\kappa=2$ & $\kappa=5$ \\
\hline 8 & 0.05030447 & 0.02832615 & 0.01549963 & 0.01663029 \\
16 & 0.00109003 & 0.00058352 & 0.00038028 & 0.00084466 \\
32 & 0.00002355 & 0.00001279 & 0.00000864 & 0.00002379 \\
64 & 0.00000002 & 0.00000001 & 0.00000001 & 0.00000004 \\
\hline \hline
\end{tabular}

Table 4.3. The Table shows the error $\left|u_{\text {calc }}-u_{\text {true }}\right|$ for the boat-shaped obstacle for several wave numbers and an increasing number of sampling points on the boundaries. Here $n$ denotes the number of sampling points on $\partial D$, i.e. $n=2 n_{1}$. Furthermore, $n_{2}$ is chosen such that $n_{2}=2 n_{1}$. 



\section{Point Source Approximation}

In this last chapter of our summary of useful tools we will deal with the approximation of point sources. For all reconstruction methods that follow the ideas of this section are crucial and the presented approximation strategies will be used as a central part in the reconstruction algorithms.

Given an obstacle $D \subset \mathbb{R}^{m}$ and a domain $\Omega$ of interest with $D \subset \Omega$ we want to find an approximating function $v_{z}$ for the point source $\Phi(\cdot, z)$ with source point $z \in \Omega \backslash \bar{D}$ on the domain $D$. We show that the single-layer potential on $\partial \Omega$ yields the designated approximation for the Helmholtz equation and also for the Laplace equation in the three dimensional space.

To enhance the compuational efficency of the point source approximation we introduce additionally a second type of approximating functions. In the case of the Helmholtz equation we consider Herglotz wave functions

$$
v_{z}(x):=\int_{\mathbb{S}^{m-1}} e^{i \kappa x \cdot d} g_{z}(d) d s(d), \quad x \in \Omega
$$

and for the Laplace equation we use some modified "static" Herglotz wave functions for the approximation. We present some strategies for the choice of appropriate approximation domains and provide a fast method for approximating the point source based on the Herglotz wave functions and a particular choice of the approximation domains.

\subsection{Approximation with a Single-Layer Potential}

This work focuses on the problem of finding a bounded inclusion $D \subset \mathbb{R}^{m}$ in a domain of interest $\Omega \supset D$, which may be bounded or equal to $\mathbb{R}^{m}$. In the first case we assume the knowledge of the field and its normal derivative on the boundary $\partial \Omega$ whereas in the second case we want to reconstruct the obstacle from the asymptotical behaviour of the field when the observation point tends to infinity. Either way the presented reconstruction algorithms are based on the approximation of a point source $\Phi(\cdot, z)$ with source point $z$ located in $\Omega \backslash \bar{D}$ on the unknown domain $D$. 
However, since the domain $D$ is to be reconstructed and hence unknown, we first have to replace the unknown approximation domain $D$ with some known domain $G(z)$. The latter may depend on the position of the source point $z$ that secondly varies in $\Omega$ rather than in $\Omega \backslash \bar{D}$. Thus, for all $z \in \Omega \backslash \bar{D}$ we choose a suitable approximation domain $G(z)$ such that $\bar{D} \subset G(z)$ and $z \notin G(z)$ for approximating the point source $\Phi(\cdot, z)$. We defer the problem of choosing the approximation domains $G(z)$ such that the admissibility condition $\bar{D} \subset G(z), z \notin G(z)$ is satisfied and assume its validity for the moment.

Let us first consider the Laplace equation in the situation that $z \in \Omega \backslash \bar{D}$ possesses an admissible approximation domain $G(z)$. We will show that we can find an approximating function $v_{z}$ in form of a single-layer potential

$$
v_{z}(x)=\int_{\partial \Omega} \Phi(x, y) \varphi_{z}(y) d s(y), \quad x \in \Omega
$$

which approximates the point source $\Phi(\cdot, z)$ on $D \subset G(z)$ in a threedimensional space. To this end we define the operator $S_{z, \Omega}: L^{2}(\partial \Omega) \longrightarrow$ $L^{2}(\partial G(z))$ by

$$
\left(S_{z, \Omega} \varphi\right)(x):=\int_{\partial \Omega} \Phi(x, y) \varphi(y) d s(y), \quad x \in \partial G(z)
$$

and prove denseness of its range provided that, additionally, $\overline{G(z)} \subset \Omega$ holds. We remark that we can avoid the last requirement by defining the single-layer potential on a boundary $\Gamma$, which contains all approximation domains $G(z)$ in its interior. Furthermore, we recall that for every bounded linear operator $A: X \longrightarrow X$ on the Hilbert space $X$ there exists a unique operator $A^{*}: X \longrightarrow X$ such that

$$
\langle A x, y\rangle=\left\langle x, A^{*} y\right\rangle
$$

for all $x, y \in X$, see for example Theorem 4.9 in [36]. The operator $A^{*}$ is called the adjoint operator to $A$.

Theorem 5.1. Let $\overline{G(z)} \subset \Omega \subset \mathbb{R}^{3}$ be a domain with boundary of class $C^{2}$. Then the operator $S_{z, \Omega}$ has dense range in $L^{2}(\partial G(z))$.

The proof follows the ideas of a similar result in [57]. To simplify notations we write $G$ instead of $G(z)$ whenever we consider a fixed source point.

Proof. To show the denseness of the range of the operator $S_{z, \Omega}$ in $L^{2}(\partial G)$ it is sufficient to prove injectivity of the adjoint operator $S_{z, \Omega}^{*}$ with respect to the scalar products

$$
\left\langle\varphi_{1}, \varphi_{2}\right\rangle=\int_{\partial \Omega} \varphi_{1} \overline{\varphi_{2}} d s, \quad \varphi_{1}, \varphi_{2} \in L^{2}(\partial \Omega)
$$


in the Hilbert space $L^{2}(\partial \Omega)$ and

$$
\left\langle\psi_{1}, \psi_{2}\right\rangle=\int_{\partial G} \psi_{1} \overline{\psi_{2}} d s, \quad \psi_{1}, \psi_{2} \in L^{2}(\partial G)
$$

in $L^{2}(\partial G)$, see Theorem 15.8 in [36]. Then the adjoint operator $S_{z, \Omega}^{*}$ of $S_{z, \Omega}$ with respect to this dual system is given by

$$
\left(S_{z, \Omega}^{*} \psi\right)(y)=\int_{\partial G} \Phi(x, y) \psi(x) d s(x), \quad y \in \partial \Omega .
$$

Let $\psi \in L^{2}(\partial G)$ be a density such that $S_{z, \Omega}^{*} \psi=0$ on $\partial \Omega$. As a consequence of Lemma 3.19

$$
u(x):=\int_{\partial G} \Phi(y, x) \psi(y) d s(y), \quad x \in \mathbb{R}^{3} \backslash \partial G
$$

is harmonic in $\mathbb{R}^{3} \backslash \bar{\Omega}$ with $\left.u\right|_{\partial \Omega} \equiv 0$. Furthermore $u(x)=o(1)$ for $|x| \rightarrow \infty$ uniformly for all directions $x /|x|$.

The uniqueness result of Theorem 2.15 for the exterior Dirichlet problem yields $u \equiv 0$ in all of $\mathbb{R}^{3} \backslash \bar{\Omega}$ and by analytic continuation $u$ vanishes in $\mathbb{R}^{3} \backslash \bar{G}$. Hence $\frac{\partial u_{+}}{\partial \nu}=0$ and the jump relations (3.52) for $L^{2}$-densities imply

$$
\left(I-K^{\prime}\right) \psi=0
$$

with the adjoint double-layer potential $K^{\prime}$ defined on $\partial G$ analogously to (3.31). We remark that both operators $K$ and $K^{\prime}$ are compact in view of Lemma 3.21. Hence, applying Fredholm's alternative as written down in Theorem 3.18 first in the dual system

$$
\left\langle C(\partial G), L^{2}(\partial G)\right\rangle, \quad\langle\tilde{\psi}, \psi\rangle:=\int_{\partial G} \tilde{\psi} \psi d s
$$

and then in the dual system

$$
\left\langle L^{2}(\partial G), L^{2}(\partial G)\right\rangle, \quad\left\langle\psi_{1}, \psi_{2}\right\rangle:=\int_{\partial G} \psi_{1} \psi_{2} d s
$$

shows that the null spaces of $I-K^{\prime}$ in $L^{2}(\partial G)$ and in $C(\partial G)$ have the same finite dimension. Since $C(\partial G)$ is a subset of $L^{2}(\partial G)$, the null spaces coincide and $\psi$ is in $C(\partial G)$.

Now continuity of the single-layer potential with continuous density implies that $u$ solves the homogeneous Dirichlet problem in $G$ and thus vanishes identically there due to the uniqueness stated in Theorem 2.15. Finally the jump relations (3.26) for the normal derivative of the single-layer potential show

$$
\psi=\frac{\partial u_{-}}{\partial \nu}-\frac{\partial u_{+}}{\partial \nu}=0
$$

This proves the injectivity of the adjoint operator $S_{z, \Omega}^{*}$ and therefore the operator $S_{z, \Omega}$ has dense range in $L^{2}(\partial G)$. 
Since the two-dimensional single-layer potential (5.8) fulfills the asymptotics $u(x)=O(1)$ only in the case when $\int_{\partial G} \varphi d s=0$, we have to choose a different approximating function. We will come back to this problem in the next section where we consider the approximation of the fundamental solution in terms of a static Herglotz wave function.

For the extension of the $L^{2}$-approximation of the point source on the boundary $\partial G$ to a uniform approximation on compact subsets of $G$ we use the concept of weak convergence in Hilbert spaces.

Definition 5.2. Let $X$ be a Hilbert space with scalar product $\langle\cdot, \cdot\rangle$. We call a sequence $\left(x_{n}\right) \subset X$ weakly convergent towards $x \in X$ if

$$
\lim _{n \rightarrow \infty}\left\langle x_{n}-x, y\right\rangle=0
$$

holds for all $y \in X$. In this case we use the notation $x_{n} \rightarrow x$ for the weak convergence of the sequence $\left(x_{n}\right)$ towards $x$.

Obviously, by an application of the Cauchy-Schwarz inequality we see that a strongly convergent sequence $\left(x_{n}\right)$ in a Hilbert space is also weakly convergent. Furthermore a weakly convergent sequence $x_{n} \rightarrow x$ is bounded. This is a consequence of the fact that we can estimate the sequence $\left(A_{n}\right)$ of bounded linear operators $A_{n}: X \longrightarrow \mathbb{C}$ defined by

$$
A_{n} y:=\left\langle x_{n}, y\right\rangle, \quad y \in X
$$

pointwise by

$$
\left\|A_{n} y\right\|=\left|\left\langle x_{n}, y\right\rangle\right| \leq\left|\left\langle x_{n}-x, y\right\rangle\right|+|\langle x, y\rangle| \leq C_{y}
$$

for all $y \in X$ due to the weak convergence $x_{n} \rightarrow x$. Then the principle of uniform boundedness, see [36], provides a uniform bound

$$
\left\|A_{n}\right\|=\sup _{\|y\|=1}\left\|A_{n} y\right\| \leq C
$$

on the sequence $\left(A_{n}\right)$ and we obtain

$$
\left\|x_{n}\right\|=\left\langle x_{n}, \frac{x_{n}}{\left\|x_{n}\right\|}\right\rangle \leq\left\|A_{n}\right\| \leq C
$$

i.e. the boundedness of $\left(x_{n}\right)$.

For any weakly convergent sequence $x_{n} \rightarrow x$ there holds

$$
\left\langle A x_{n}-A x, y\right\rangle=\left\langle x_{n}-x, A^{*} y\right\rangle \rightarrow 0
$$

for all $y \in X$ when $n \rightarrow \infty$. Thus a bounded linear operator on a Hilbert space maps weakly convergent sequences on weakly convergent sequences, more precisely the weak convergence $x_{n} \rightarrow x$ implies $A x_{n} \rightarrow A x$. Moreover if we assume the operator $A$ to be compact and the sequence $\left(x_{n}\right)$ to be 
weakly convergent towards $x$ then we can prove the strong convergence of the sequence of images $\left(A x_{n}\right)$ towards $A x$. Since, assuming that $A x_{n} \rightarrow A x$ does not hold, there exists an $\varepsilon>0$ and a subsequence $x_{n_{k}}$ such that

$$
\left\|A x_{n_{k}}-A x\right\| \geq \varepsilon
$$

for all $k \in \mathbb{N}$. However, the weak convergence of $\left(x_{n}\right)$ implies the boundedness of the subsequence $\left(x_{n_{k}}\right)$ and from the compactness of the operator $A$ we obtain the existence of a convergent subsequence of $A x_{n_{k}}$ with limit $A x$, which contradicts (5.19).

Now, we are well prepared to formulate and proof the following lemma which guarantees the extension of the $L^{2}$-approximation of the point source on the boundary of the approximation domain to its interior in the sense of uniform convergence on compact subsets.

Lemma 5.3. Let $D \subset \mathbb{R}^{m}$ be a bounded domain with $C^{2}$-boundary and let $u_{n}$ be a sequence of $C^{2}(D) \cap C(\bar{D})$ solutions to the Laplace equation in $D$ such that the boundary data $f_{n}=\left.u_{n}\right|_{\partial D}$ are weakly convergent in $L^{2}(\partial D)$. Then the sequence $u_{n}$ converges uniformly on compact subsets of $D$ to a solution of the Laplace equation.

Proof. We adapt the proof of the analoguous result for the Helmholtz equation which is given in [7].

For any $\psi \in C(\partial D)$ the double-layer potential

$$
v(x)=\int_{\partial D} \frac{\partial \Phi(x, y)}{\partial \nu(y)} \psi(y) d s(y)
$$

defines a harmonic function in $\mathbb{R}^{m} \backslash \partial D$. In particular $v$ is a solution to the interior Dirichlet boundary value problem with boundary data $f \in C(\partial D)$ if $\psi$ is a solution to the integral equation

$$
(I-K) \psi=-2 f
$$

on $\partial D$. We prove the injectivity of the operator $I-K$ and apply the theorem of Riesz. Let $\psi \in C(\partial D)$ be a solution to (5.21) with boundary data $f=0$. Then $v$ solves the homogeneous Dirichlet problem in $D$ and by the uniqueness Theorem 2.15 of this problem $v$ vanishes in $D$. From (3.28) and $\frac{\partial v_{-}}{\partial \nu}=0$ together with the fact that $v=o(1)$ it follows that $v$ solves the exterior Neumann problem for the Laplace equation. Again Theorem 2.15 provides the uniqueness of this problem and implies that $v$ also vanishes in $\mathbb{R}^{m} \backslash \bar{D}$. Finally we obtain

$$
\psi=v_{+}-v_{-}=0
$$

from the jump relations (3.27). Hence the operator $I-K$, which is a compact perturbation of the identity, is injective and therefore it possesses a bounded 
inverse due to the Theorem 3.14 of Riesz. Thus we have proven the unique solvability of the interior Dirichlet problem for the Laplace equation.

In particular the above argumentation shows that we can represent each function $u_{n}$ as a double-layer potential with the continuous density

$$
\psi_{n}=-2(I-K)^{-1} f_{n} .
$$

In the next step we apply Fredholm's alternative as in the previous proof to show the boundedness of $(I-K)^{-1}$ from $L^{2}(\partial D)$ into $L^{2}(\partial D)$. To this end we remark that the integral operator $K: L^{2}(\partial D) \longrightarrow L^{2}(\partial D)$ is compact in view of Lemma 3.21. We apply Fredholm's alternative 3.18 first in the dual system

$$
\left\langle C(\partial D), L^{2}(\partial D)\right\rangle, \quad\langle\psi, \varphi\rangle:=\int_{\partial D} \psi \varphi d s
$$

and then in the dual system

$$
\left\langle L^{2}(\partial D), L^{2}(\partial D)\right\rangle, \quad\left\langle\varphi_{1}, \varphi_{2}\right\rangle:=\int_{\partial D} \varphi_{1} \varphi_{2} d s
$$

to deduce that the null spaces of $I-K$ in $L^{2}(\partial D)$ and in $C(\partial D)$ have the same finite dimension. Since $C(\partial D)$ is a subset of $L^{2}(\partial D)$, the null spaces coincide and we obtain the injectivity of the compact operator

$$
I-K: L^{2}(\partial D) \longrightarrow L^{2}(\partial D) \text {. }
$$

Hence the Theorem 3.14 of Riesz endows the boundedness of the inverse operator $(I-K)^{-1}$ in $L^{2}(\partial D)$.

Now the weak convergence of the boundary data $f_{n} \rightarrow f$ in $L^{2}(\partial D)$ and the boundedness of the inverse operator imply the weak convergence of the $L^{2}$ densities $\varphi_{n}=-2(I-K)^{-1} f_{n}$ towards $\varphi=-2(I-K)^{-1} f$. Therefore the sequence of double-layer potentials $u_{n}$ with densities $\varphi_{n}$ converge pointwise in $D$ to the double-layer potential $u$ with density $\varphi$. The Cauchy-Schwarz inequality applied to the double-layer potential yields

$$
\left|u_{n}\left(x_{1}\right)-u_{n}\left(x_{2}\right)\right| \leq|\partial D|^{\frac{1}{2}} \sup _{y \in \partial D}\left|\frac{\partial \Phi\left(x_{1}, y\right)}{\partial \nu(y)}-\frac{\partial \Phi\left(x_{2}, y\right)}{\partial \nu(y)}\right|\left\|\varphi_{n}\right\|_{L^{2}(\partial D)}
$$

for all $x_{1}, x_{2} \in D$. Together with the boundedness of the weakly convergent sequence $\left(\varphi_{n}\right)$ this implies the equicontinuity of $u_{n}$ on compact subsets of $D$. Finally we obtain the uniform convergence of $\left(u_{n}\right)$ on compact subsets of $D$ from the pointwise convergence and the equicontinuity of this sequence.

A combination of Theorem 5.1 and Lemma 5.3 yields the uniform convergence of the approximation $v_{z}$ of the point source $\Phi(\cdot, z)$ on $\bar{D}$ as follows. Consider a source point $z \in \Omega \backslash \bar{D}$ with admissible approximation domain 
$\overline{G(z)} \subset \Omega$, i.e. $z \notin \overline{G(z)}$ and $\bar{D} \subset G(z)$. By Theorem 5.1 there exists a density $\varphi_{z, \varepsilon}$ such that

$$
\left\|S_{z, \Omega} \varphi_{z, \varepsilon}-\left.\Phi(\cdot, z)\right|_{\partial G}\right\|_{L^{2}(\partial G)} \leq \varepsilon
$$

for any given $\varepsilon>0$. Therefore the boundary data of the harmonic functions

$$
u_{\varepsilon}(x):=\int_{\partial \Omega} \Phi(x, y) \varphi_{z, \varepsilon}(y) d s(y)-\Phi(x, z), \quad x \in G(z)
$$

converge to zero in $L^{2}(\partial G)$ for $\varepsilon \rightarrow 0$. The previous lemma together with the uniqueness of the interior Dirichlet problem implies the uniform convergence $u_{\varepsilon} \rightarrow 0$ on compact subsets of $G(z)$. Since $\bar{D} \subset G(z)$ we obtain the uniform convergence $v_{z, \varepsilon} \rightarrow \Phi(\cdot, z)$ on $\bar{D}$ with

$$
v_{z, \varepsilon}:=\int_{\partial \Omega} \Phi(x, y) \varphi_{z, \varepsilon}(y) d s(y), \quad x \in G(z) .
$$

We note that Lemma 5.3 can be easily generalized to furnish the uniform convergence of the derivatives, too. Hence we have proven the following corollary.

Corollary 5.4. For any $z \in \Omega \backslash \bar{D}$ with admissible approximation domain $\overline{G(z)} \subset \Omega$ the point source $\Phi(\cdot, z)$ together with its derivatives can be approximated uniformly on $\bar{D}$ by functions $v_{z, \varepsilon}$ of the form (5.30).

We remark that the equation

$$
S_{z, \Omega} \varphi=\left.\Phi(\cdot, z)\right|_{\partial G(z)}
$$

is not solvable in $L^{2}(\partial \Omega)$. Assume the existence of a function $\varphi \in L^{2}(\partial \Omega)$ solving the above equation. Then

$$
u(x):=\int_{\partial \Omega} \Phi(x, y) \varphi(y) d s(y)-\Phi(x, z), \quad x \in \Omega \backslash\{z\},
$$

is in the space $C^{2}(G) \cap C(\bar{G})$, see Lemma 3.19, and solves the Laplace equation in $G$ with boundary data $u=0$ on $\partial G$. By uniqueness, $u$ has to vanish in all of $G$ first and then $u$ vanishes in all of $\Omega \backslash\{z\}$ due to analytic continuation. But this contradicts the unboundedness of $u$ in any neigbourhood of $z$ in view of the singularity of the fundamental solution $\Phi(\cdot, z)$ in $z$.

Moreover, the integral operator $S_{z, \Omega}$ is compact from $L^{2}(\partial \Omega)$ into $L^{2}(\partial G)$. As a consequence the approximation of a point source via (5.30) results in the ill-posed problem of solving an integral equation of the first kind. For a stable approximate solution $\varphi$ of (5.31) we therefore have to apply a regularization technique as for example the Tikhonov regularization. To this end we have to verify the injectivity of $S_{z, \Omega}$ and the denseness of the 
range $S_{z, \Omega}\left(L^{2}(\partial \Omega)\right)$ in $L^{2}(\partial G)$, which guarantees the uniqueness and the existence of an approximate solution respectively. In Theorem 5.1 we have already validated the denseness property of this single-layer operator. We now prove its injectivity in the three-dimensional space.

Lemma 5.5. The operator $S_{z, \Omega}: L^{2}(\partial \Omega) \longrightarrow L^{2}(\partial G(z))$ is injective.

Proof. Let $\varphi \in L^{2}(\partial \Omega)$ such that $S_{z, \Omega} \varphi=0$. Then the function

$$
u(x)=\int_{\partial \Omega} \Phi(x, y) \varphi(y) d s(y), \quad x \in \mathbb{R}^{3} \backslash \partial \Omega
$$

solves the homogeneous Dirichlet problem for the Laplace equation in $G(z)$ and the uniqueness of this problem proves that $u$ vanishes there. By analytic continuation $u$ vanishes in all of $\Omega$ and the behaviour (3.51) of $u$ at the boundary $\partial \Omega$ implies that $u$ solves the exterior Dirichlet problem, too.

Again the uniqueness property of this problem shows that $u$ vanishes in $\mathbb{R}^{3} \backslash \bar{\Omega}$ and then the jump relations (3.52) for $L^{2}$ densities yield $\varphi=0$.

Hence, we can apply a Tikhonov regularization scheme to calculate an approximating density $\varphi$ to approximate the point source $\Phi(\cdot, z)$ in $G$ in terms of a single-layer potential in the following way. First we substitute the illposed integral equation

$$
S_{z, \Omega} \varphi=\left.\Phi(\cdot, z)\right|_{\partial G(z)}
$$

of the first kind with its regularized version

$$
\left(\alpha I+S_{z, \Omega}^{*} S_{z, \Omega}\right) \varphi=\left.S_{z, \Omega}^{*} \Phi(\cdot, z)\right|_{\partial G(z)} .
$$

Then we compute an approximate solution

$$
\varphi_{z, \varepsilon}=\left.\left(\alpha I+S_{z, \Omega}^{*} S_{z, \Omega}\right)^{-1} S_{z, \Omega}^{*} \Phi(\cdot, z)\right|_{\partial G(z)}
$$

to (5.34) that satisfies

$$
\left\|v_{z, \varepsilon}-\Phi(\cdot, z)\right\|_{C^{1}(\bar{D})} \leq \varepsilon
$$

by choosing $\alpha=\alpha(\varepsilon)$ appropriately. The existence of an $\alpha>0$ with this property is ensured with the following lemma.

Lemma 5.6. Let $z \in \Omega \backslash \bar{D}$ be a source point with admissible approximation domain $\overline{G(z)} \subset \Omega$. Then for every $\varepsilon>0$ there exist an $\alpha>0$ such that (5.37) holds with $v_{z, \varepsilon}$ and $\varphi_{z, \varepsilon}$ defined in (5.30) and (5.36).

Proof. By Theorem 4.15 in [7] there exists for every $\delta>0$ small enough a unique regularization parameter $\alpha=\alpha(\delta)>0$ such that

$$
\left\|S_{z, \Omega} \varphi_{\alpha}-\Phi(\cdot, z)\right\|_{L^{2}(\partial G)}=\delta
$$


with $\varphi_{\alpha}$ being the unique solution to (5.35). Let $\left(\delta_{n}\right)$ be a sequence of postive numbers such that $\delta_{n} \rightarrow 0$ for $n \rightarrow \infty$. Then the boundary data $f_{n}:=S_{z, \Omega} \varphi_{n}$ converges to zero in $L^{2}(\partial G)$, where we have used the notation $\varphi_{n}=\varphi_{\alpha\left(\delta_{n}\right)}$. By the uniform convergence of

$$
v_{n}(x)=\int_{\partial \Omega} \Phi(x, y) \varphi_{n}(y) d s(y)
$$

to $\Phi(\cdot, z)$ in $\bar{D}$ we obtain the existence of an integer $n_{0}$ such that

$$
\left\|v_{n}-\Phi(\cdot, z)\right\|_{C^{1}(\bar{D})} \leq \varepsilon
$$

holds for all $n \geq n_{0}$. Hence the choice $\alpha=\alpha\left(\delta_{n_{0}}\right)$ is suitable.

Studying the proofs of the previous theorems shows that this construction of the approximating function $v_{z}$ to the fundamental solution of Laplace's equation can be easily transferred to the case of the Helmholtz equation. We summarize the analogon of Theorem 5.1, Lemma 5.3 and Lemma 5.5 in the next theorem.

Theorem 5.7. Let $\bar{G} \subset \Omega$ be a domain such that $\bar{D} \subset G$ and that $-\kappa^{2}$ is not a Dirichlet eigenvalue for the Laplacian in $G$. Furthermore, let $\Phi$ be the fundamental solution to the Helmholtz equation and let $z \in \Omega \backslash \bar{G}$. Then, the operator $S_{z, \Omega}: L^{2}(\partial \Omega) \longrightarrow L^{2}(\partial G)$ defined by

$$
\left(S_{z, \Omega} \varphi\right)(x)=\int_{\partial \Omega} \Phi(x, y) \varphi(y) d s(y), \quad x \in \partial G
$$

is injective and has dense range.

If the boundary data $f_{n}=\left.u_{n}\right|_{\partial G}$ of a sequence $u_{n} \in C^{2}(G) \cap C(\bar{G})$ of solutions to the Helmholtz equation in $G$ are weakly convergent in $L^{2}(\partial G)$, then the sequence $u_{n}$ converges uniformly on compact subsets of $G$ to a solution of the Helmholtz equation.

Since the proof of this theorem is essentially the same as the proofs for the corresponding theorem and lemmas for the Laplace equation we leave it out here and refer the reader to [14] for the details.

The computation of several approximations $v_{z_{j}}$ for point sources $\Phi\left(\cdot, z_{j}\right)$ with source points $z_{j}$ chosen from a set of sampling points

$$
\mathcal{S}:=\left\{z_{j} \in \Omega: j=1, \ldots, N\right\}
$$

requires the solution of $N$ ill-posed integral equations of the first kind. In general, we have to adjust the domain of approximation for each source point in order to satisfy the admissibility condition and we call the set

$$
\mathcal{G}=\{G(z): z \in \mathcal{S}\}
$$


a strategy for the choice of the approximation domains. Hence the integral operator on the left side of (5.35) depends on the domain $G\left(z_{j}\right)$ and hereby on the source point $z_{j}$. Therefore we have to set up both the right hand and the left hand side in each step for solving the regularized equation (5.35). In the next sections we will consider a different approximation scheme for a special strategy for the choice of the approximation domains which will reduce the computational costs arising from the approximation of $\Phi(\cdot, z)$ for all $z \in \mathcal{S}$.

\subsection{Approximation with the Herglotz Wave Operator}

The main drawback of the approximation of the fundamental solution with a single-layer potential is the computational cost of this operation since we have to apply a Tikhonov regularization scheme to the ill-posed integral equation (5.34) for each sampling point $z \in \mathcal{S}$. Therefore we try to find an approximation of a point source with another type of function which solves the underlying partial differential equation. For the Helmholtz equation a superposition of plane waves

$$
v(x)=\int_{\mathbb{S}^{m-1}} e^{i \kappa x \cdot d} g(d) d s(d), \quad x \in \mathbb{R}^{m},
$$

which is also called a Herglotz wave function seems to be a promising candidate. Herglotz wave functions are entire solutions to the Helmholtz equation, i.e. they solve Helmholtz' equation in $\mathbb{R}^{m}$, and have some further nice denseness properties. In particular, we can state the following analogon to Theorem 5.7.

Theorem 5.8. Let $G \subset \mathbb{R}^{m}$ be a bounded domain with boundary of class $C^{2}$ such that $-\kappa^{2}$ is not a Dirichlet eigenvalue for the Laplacian in $G$. Then, the Herglotz wave operator $H: L^{2}\left(\mathbb{S}^{m-1}\right) \longrightarrow L^{2}(\partial G)$ defined by

$$
(H g)(x)=\int_{\mathbb{S}^{m-1}} e^{i \kappa x \cdot d} g(d) d s(d), \quad x \in \partial G
$$

is injective and has dense range.

We pass on the proof which is given for example in [7], Theorem 5.24, and turn to the problem of defining a Herglotz wave operator in the static case $\kappa=0$, i.e. we are looking for some dense Herglotz wave like functions for the Laplace equation.

Obviously, a simple limit process $\kappa \rightarrow 0$ will not result in nice "static" plane waves since the limit

$$
\lim _{\kappa \rightarrow 0} e^{i \kappa x \cdot d}=1
$$

leads to a constant function which will not be of any use in defining a dense "static Herglotz wave operator". However, Gen Nakamura suggested in a 
private communication to use a kernel of the form $k(x, d)=e^{i\left(i d+d^{\perp}\right) \cdot x}$ for the definition of a static Herglotz wave operator. Following his idea we define the static Herglotz wave operator $H: L^{2}\left(\mathbb{S}^{1}\right) \rightarrow L^{2}(\partial G)$ in two dimensions by

$$
(H g)(x):=\int_{\mathbb{S}^{1}} \operatorname{Re}\left(e^{i\left(i d+d^{\perp}\right) \cdot x}\right) g(d) d s(d)
$$

with a real density $g \in L^{2}\left(\mathbb{S}^{1}\right)$ and $d^{\perp}:=\left(d_{2},-d_{1}\right)$. Furthermore we call a function $v$ of the form

$$
v(x)=\operatorname{Re} \int_{\mathbb{S}^{1}} e^{i\left(i d+d^{\perp}\right) \cdot x} g(d) d s(d), \quad x \in \mathbb{R}^{2}
$$

with a possibly complex density $g \in L^{2}\left(\mathbb{S}^{1}\right)$ a static Herglotz wave function.

We prove the injectivity and denseness property of the static operator to provide the missing two-dimensional approximation of the fundamental solution in the static case. We do this by adapting the proof of the injectivity and denseness of the Herglotz wave operator as given in [7] for the threedimensional case.

To this end we need the Funk-Hecke formula in two dimensions.

Theorem 5.9 (Funk-Hecke formula). Let $f$ be a continuous function on the interval $[-1,1]$. Then for every spherical harmonic $Y_{n}$ of degree $n$ there holds the Funk-Hecke formula

$$
\int_{\mathbb{S}^{1}} f(\hat{x} \cdot d) Y_{n}(d) d s(d)=\lambda Y_{n}(\hat{x}), \quad \hat{x} \in \mathbb{S}^{1}
$$

with

$$
\lambda=2 \int_{-1}^{1} f(t) \frac{P_{n}(t)}{\sqrt{1-t^{2}}} d t
$$

and the Chebychev polynomials of degree $n$ given by Rodrigues' formula

$$
P_{n}(t)=\left(-\frac{1}{2}\right)^{n} \frac{\Gamma\left(\frac{1}{2}\right)}{\Gamma\left(n+\frac{1}{2}\right)} \sqrt{1-t^{2}} \frac{d^{n}}{d t^{n}}\left(1-t^{2}\right)^{n-\frac{1}{2}} .
$$

For a proof of the Funk-Hecke formula and the formula of Rodrigues we refer the reader to [47]. This reference may also serve as an introduction into spherical harmonics in arbitrary dimensions.

We remark that if we replace the integration domain by $\mathbb{S}^{m-1}$ and the constant $\lambda$ by

$$
\lambda=\omega_{m-1} \int_{-1}^{1} f(t) P_{n}(t)\left(1-t^{2}\right)^{\frac{m-3}{2}} d t,
$$

we obtain the Funk-Hecke formula for the general case $\mathbb{R}^{m}, m>2$. Here $\omega_{m-1}=\left|\mathbb{S}^{m-2}\right|$ denotes the area of the unit sphere in $m-1$ dimensions and the polynomials $P_{n}$ are the Legendre polynomials of degree $n$. Furthermore 
the formula of Rodrigues for the Legendre polynomials in $m$ dimensions has the form

$$
P_{n}(t)=\left(-\frac{1}{2}\right)^{n} \frac{\Gamma\left(\frac{m-1}{2}\right)}{\Gamma\left(n+\frac{m-1}{2}\right)}\left(1-t^{2}\right)^{\frac{3-m}{2}} \frac{d^{n}}{d t^{n}}\left(1-t^{2}\right)^{n+\frac{m-3}{2}} .
$$

Before we study the static Herglotz wave operator in more detail we need a preparing lemma.

Lemma 5.10. For every $n \in \mathbb{N}$ there exists an $\varepsilon>0$ such that

$$
\lambda_{n}(r):=\int_{-1}^{1} \frac{d^{n}}{d t^{n}}\left\{\left(1-t^{2}\right)^{n-\frac{1}{2}}\right\} e^{-r t} \cos r \sqrt{1-t^{2}} d t>0
$$

for all $r$ in the open interval $(0, \varepsilon)$.

Proof. We consider the Taylor expansion of $\lambda_{n}(r)$ in $r=0$. To this end we apply Leibniz' product rule to calculate the $m$-th derivative of $\lambda_{n}$ with respect to $r$ which yields

$$
\lambda_{n}^{(m)}(r)=\sum_{k=0}^{m}\left(\begin{array}{c}
m \\
k
\end{array}\right) \int_{-1}^{1} \frac{d^{n}}{d t^{n}}\left\{\left(1-t^{2}\right)^{n-\frac{1}{2}}\right\} \frac{d^{m-k}}{d r^{m-k}} e^{-r t} \frac{d^{k}}{d r^{k}} \cos r \sqrt{1-t^{2}} d t
$$

where the derivatives in $r=0$ are given by

$$
\left.\frac{d^{m-k}}{d r^{m-k}} e^{-r t}\right|_{r=0}=(-t)^{m-k}
$$

and

$$
\left.\frac{d^{k}}{d r^{k}} \cos r \sqrt{1-t^{2}}\right|_{r=0}=\left\{\begin{array}{cl}
0 & , \text { for } \mathrm{k} \text { odd } \\
(-1)^{\frac{k}{2}}\left(1-t^{2}\right)^{\frac{k}{2}}, & \text { for } \mathrm{k} \text { even }
\end{array}\right.
$$

Hence we obtain

$$
\lambda_{n}^{(m)}(0)=\sum_{\substack{k=0, k \text { even }}}^{m}(-1)^{m-\frac{k}{2}}\left(\begin{array}{c}
m \\
k
\end{array}\right) \int_{-1}^{1} \frac{d^{n}}{d t^{n}}\left\{\left(1-t^{2}\right)^{n-\frac{1}{2}}\right\}\left(1-t^{2}\right)^{\frac{k}{2}} t^{m-k} d t
$$

and evaluate the remaining integral with an $n$-fold partial integration

$$
\begin{aligned}
& \int_{-1}^{1} \frac{d^{n}}{d t^{n}}\left\{\left(1-t^{2}\right)^{n-\frac{1}{2}}\right\}\left(1-t^{2}\right)^{\frac{k}{2}} t^{m-k} d t \\
& =(-1)^{n} \int_{-1}^{1}\left(1-t^{2}\right)^{n-\frac{1}{2}} \frac{d^{n}}{d t^{n}}\left\{\left(1-t^{2}\right)^{\frac{k}{2}} t^{m-k}\right\} d t,
\end{aligned}
$$

where we have made use of the fact that both $t=1$ and $t=-1$ are zeros of order $n-1$ of the function $\left(1-t^{2}\right)^{n-\frac{1}{2}}$. Since $k$ is even, the function 
$\left(1-t^{2}\right)^{\frac{k}{2}} t^{m-k}$ is a polynomial of degree $m$ with leading coefficient $a_{m}=$ $(-1)^{\frac{k}{2}}$. Therefore $\lambda_{n}^{(m)}(0)=0$ for $m<n$ and for $m=n$ we calculate

$$
\begin{aligned}
\lambda_{n}^{(n)}(0) & =\sum_{\substack{k=0, k \text { even }}}^{n}(-1)^{n-\frac{k}{2}}\left(\begin{array}{l}
n \\
k
\end{array}\right)(-1)^{n} \int_{-1}^{1}\left(1-t^{2}\right)^{n-\frac{1}{2}} n !(-1)^{\frac{k}{2}} d t \\
& =\sum_{\substack{k=0, k \text { even }}}^{n}\left(\begin{array}{l}
n \\
k
\end{array}\right) n ! \int_{-1}^{1}\left(1-t^{2}\right)^{n-\frac{1}{2}} d t>0 .
\end{aligned}
$$

From the Taylor expansion we conclude the asymptotics

$$
\lambda_{n}(r)=\sum_{\substack{k=0, k \text { even }}}^{n}\left(\begin{array}{l}
n \\
k
\end{array}\right) \int_{-1}^{1}\left(1-t^{2}\right)^{n-\frac{1}{2}} d t r^{n}+o\left(r^{n}\right), \quad r>0,
$$

for $r \rightarrow 0$ which implies the existance of an $\varepsilon>0$ such that $\lambda_{n}(r)>0$ in the open interval $(0, \varepsilon)$.

With the Funk-Hecke formula we can easily prove that the basic properties of the Herglotz wave operator carry over to the static case. One easily verifies that $v=H g$ is an entire solution to the Laplace equation by direct calculation. Moreover this operator is injective and has dense range.

Theorem 5.11. The static Herglotz wave operator is injective. If, in addition, $G$ is a circular domain, then the static Herglotz wave operator has dense range.

Proof. To prove the injectivity of the operator $H$ we assume that the real density $g \in L^{2}\left(\mathbb{S}^{1}\right)$ satisfies $H g=0$ on $\partial G$. Then the harmonic function

$$
u(x)=\int_{\mathbb{S}^{1}} \operatorname{Re}\left(e^{i\left(i d+d^{\perp}\right) \cdot x}\right) g(d) d s(d), \quad x \in \mathbb{R}^{2}
$$

solves the interior Dirichlet problem in $G$ with vanishing boundary data. By the maximum principle for harmonic functions $u$ vanishes in all of $G$. Hence, the unique continuation principle yields that $u$ is zero everywhere. For $x \in \mathbb{R}^{2} \backslash\{0\}$ the orthogonal decomposition

$$
x=(x \cdot d) d+\left(x \cdot d^{\perp}\right) d^{\perp}
$$

of $x$ shows that

$$
\left(x \cdot d^{\perp}\right)^{2}=r^{2}\left(1-(\hat{x} \cdot d)^{2}\right)
$$

with $\hat{x}=\frac{x}{|x|}$ and with $r=|x|$. Together with the symmetry of the cosine function we obtain

$$
\cos \left(x \cdot d^{\perp}\right)=\cos \left|x \cdot d^{\perp}\right|=\cos r \sqrt{1-(\hat{x} \cdot d)^{2}} .
$$


Thus the kernel of the static Herglotz wave function is given by

$$
\operatorname{Re} e^{i\left(i d+d^{\perp}\right) \cdot x}=e^{-r d \cdot \hat{x}} \cos r \sqrt{1-(\hat{x} \cdot d)^{2}}, \quad x=r \hat{x} \in \mathbb{R}^{2} \backslash\{0\} .
$$

We consider $x$ on circles $\partial B_{r}(0)$ of radius $r$ and apply the Funk-Hecke formula (5.49), where we choose the continuous function

$$
f(t)=f_{r}(t):=e^{-r t} \cos r \sqrt{1-t^{2}}
$$

to obtain

$$
\int_{\mathbb{S}^{1}} f(\hat{x} \cdot d) Y_{n}(d) d s(d)=\lambda_{n} Y_{n}(\hat{x}), \quad \hat{x} \in \mathbb{S}^{1}
$$

with $\lambda_{n}$ defined in (5.50). In particular $\lambda_{n}$ is of the form

$$
\lambda_{n}=c_{n} \int_{-1}^{1} \frac{d^{n}}{d t^{n}}\left\{\left(1-t^{2}\right)^{n-\frac{1}{2}}\right\} e^{-r t} \cos r \sqrt{1-t^{2}} d t
$$

with some constant $c_{n} \neq 0$. Now for given $n \in \mathbb{N}$ Lemma 5.10 provides the existence of an $r>0$ such that $\lambda_{n} \neq 0$ and we can divide equation (5.68) by $\lambda_{n}$. Multiplying the resulting equation with $g(\hat{x})$ and integrating both sides over the unit sphere with respect to $\hat{x}$ leads to

$$
\frac{1}{\lambda_{n}} \int_{\mathbb{S}^{1}} g(\hat{x}) \int_{\mathbb{S}^{1}} f(\hat{x} \cdot d) Y_{n}(d) d s(d) d s(\hat{x})=\int_{\mathbb{S}^{1}} Y_{n}(\hat{x}) g(\hat{x}) d s(\hat{x}) .
$$

Interchanging the order of integration on the left hand side and using

$$
u(x)=\int_{\mathbb{S}^{1}} f(\hat{x} \cdot d) g(d) d s(d)=0, \quad x=r \hat{x} \in \partial B_{r}(0),
$$

we derive

$$
\int_{\mathbb{S}^{1}} Y_{n}(\hat{x}) g(\hat{x}) d s(\hat{x})=\frac{1}{\lambda_{n}} \int_{\mathbb{S}^{1}} Y_{n}(d) \int_{\mathbb{S}^{1}} f(\hat{x} \cdot d) g(\hat{x}) d s(\hat{x}) d s(d)=0 .
$$

The completeness of the spherical harmonics in $L^{2}\left(\mathbb{S}^{1}\right)$ proves that $g=0$ and hereby the injectivity of the operator $H$.

Let us now assume that $G$ is a circular domain. We demonstrate that the operator $H$ has dense range by verifying the injectivity of the adjoint operator $H^{*} L^{2}(\partial G) \longrightarrow L^{2}\left(\mathbb{S}^{1}\right)$ which is given by

$$
\left(H^{*} \varphi\right)(d)=\int_{\partial G} \operatorname{Re}\left(e^{i\left(i d+d^{\perp}\right) \cdot x}\right) \varphi(x) d s(x), \quad d \in \mathbb{S}^{1} .
$$

Let $\varphi \in L^{2}(\partial G)$ be a real-valued density such that $H^{*} \varphi=0$ on the unit sphere. From $y^{\perp} \cdot x=-y \cdot x^{\perp}$ we obtain

$$
\Delta_{y} e^{i\left(i y+y^{\perp}\right) \cdot x}=\Delta_{y} e^{i\left(i x-x^{\perp}\right) \cdot y}=-\left(i x-x^{\perp}\right) \cdot\left(i x-x^{\perp}\right) e^{i\left(i x-x^{\perp}\right) \cdot y}=0
$$


for all $y \in \mathbb{R}^{2}$, i.e. the function

$$
v(y)=\int_{\partial G} \operatorname{Re}\left(e^{i\left(i y+y^{\perp}\right) \cdot x}\right) \varphi(x) d s(x), \quad y \in \mathbb{R}^{2}
$$

is harmonic in $\mathbb{R}^{2}$ and vanishes on the unit sphere. As before we apply the maximum-minimum principle for harmonic functions together with an analytic continuation argument to show that $v$ vanishes in all of $\mathbb{R}^{2}$. Since $G$ is a circular domain we assume the boundary $\partial G$ to be parametrized by $x=r_{0} d$ with $d \in \mathbb{S}^{1}$ and constant radius $r_{0}>0$. Hence we can transform the integration contour $\partial G$ onto the unit sphere and obtain the representation

$$
v(y)=\int_{\mathbb{S}^{1}} \operatorname{Re}\left(e^{i\left(i y+y^{\perp}\right) \cdot d r_{0}}\right) \tilde{\varphi}(d) d s(d), \quad y \in \mathbb{R}^{2}
$$

for $v$, where we have used the notation

$$
\tilde{\varphi}(d)=\varphi\left(r_{0} d\right) r_{0}
$$

Again we use the orthogonal decomposition

$$
d=\frac{1}{|y|^{2}}\left((d \cdot y) y+\left(d \cdot y^{\perp}\right) y^{\perp}\right)
$$

for $y \neq 0$ to conclude

$$
\left(d \cdot y^{\perp}\right)^{2}=|y|^{2}-(d \cdot y)^{2}, \quad y \in \mathbb{R}^{2} .
$$

Therefore $v$ can be written as

$$
v(y)=\int_{\mathbb{S}^{1}} e^{-r_{0} y \cdot d} \cos \left(r_{0} \sqrt{|y|^{2}-(d \cdot y)^{2}}\right) \tilde{\varphi}(d) d s(d), \quad y \in \mathbb{R}^{2} .
$$

Now we consider $y$ on circles $\partial B_{r / r_{0}}$ and conclude $\tilde{\varphi}(d)=0$ with the same argumentation as before. Since $r_{0}$ is positive we finally obtain

$$
\varphi\left(r_{0} d\right)=\frac{\tilde{\varphi}(d)}{r_{0}}=0
$$

for all $x=r_{0} d \in \partial G$.

Analogously to the approximation of the point source with a single-layer potential we can approximate the point source $\Phi(\cdot, z)$ with source point $z \in \mathcal{S}$ with a (static) Herglotz wave function defined in (5.44) and (5.48). Since the Herglotz wave operators are injective with dense range we can apply a Tikhonov regularization scheme and proceed as before. In particular we solve the regularized integral equation

$$
\left(\alpha I+H^{*} H\right) g_{z, \varepsilon}=\left.H^{*} \Phi(\cdot, z)\right|_{\partial G(z)}
$$


for the density $g_{z, \varepsilon} \in L^{2}\left(\mathbb{S}^{m-1}\right)$ of the approximating Herglotz wave function such that

$$
\left\|v_{z, \varepsilon}-\Phi(\cdot, z)\right\|_{C^{1}(\bar{D})} \leq \varepsilon
$$

Here, $v_{z, \varepsilon}$ is given by

$$
v_{z, \varepsilon}(x)=\int_{\mathbb{S}^{1}} e^{i \kappa x \cdot d} g_{z, \varepsilon}(d) d s(d), \quad x \in \mathbb{R}^{m}
$$

for the Helmholtz equation and by

$$
v_{z, \varepsilon}(x)=\int_{\mathbb{S}^{1}} \operatorname{Re}\left(e^{i\left(i d+d^{\perp}\right) \cdot x}\right) g_{z, \varepsilon}(d) d s(d), \quad x \in \mathbb{R}^{2}
$$

in the static case. Furthermore the existence of an appropriate regularization parameter $\alpha=\alpha(\varepsilon)$ is guaranteed by Lemma 5.6.

In the following we demonstrate a fast method for the approximation of a point source with source point varying in $\mathcal{S}$ based on the Herglotz wave functions (5.44) and (5.48). To this end we will introduce a special strategy for the choice of the approximation domains first.

\subsection{Choice of Approximation Domains}

For the approximation of a point source $\Phi(\cdot, z)$ with source point $z \in \mathcal{S}$ on an arbitrary but admissible approximation domain $G(z)$ we have to solve the regularized integral equation (5.82) for the density $g_{z, \varepsilon}$ of the approximating Herglotz wave function. If we want to calculate an approximating function for every $z \in \mathcal{S}=\left\{z_{1}, \ldots, z_{N}\right\}$ we have to solve $N$ different regularized equations since both the left hand and the right hand side will vary for an arbitrary choice of the approximation domains $G(z)$.

Let us now consider a fixed configuration of the source point and its corresponding approximation domain, i.e. we assume that the geometrical figures $\mathcal{F}_{j}:=\left\{z_{j}, G\left(z_{j}\right)\right\}, j=1, \ldots, N$, are congruent with each other. Therefore we obtain the representation

$$
G\left(z_{j}\right)=\left\{M\left(z_{j}\right) x+z_{j}: x \in G_{0}, M\left(z_{j}\right) \in \mathrm{SO}(m, \mathbb{R})\right\}
$$

for the approximation domain $G\left(z_{j}\right)$ with the reference approximation domain $G_{0}:=G(0)$ such that $0 \notin G_{0}$. Here $\mathrm{SO}(m, \mathbb{R})$ is the set of real, orthogonal $m \times m$ matrices with $\operatorname{det}(M)=1$. We remark that $G\left(z_{j}\right)$ emerges from $G_{0}$ by first rotating the domain $G_{0}$ and then translating it by the vector $z_{j}$. Since $0 \notin G_{0}$ all approximation domains fulfill the condition $z_{j} \notin G\left(z_{j}\right)$, but in general they are not admissible. Seeking for an approximation on the unknown domain $D$ we will therefore need some indicator function for identifying the figures $\mathcal{F}_{j}$, which satisfy additionally $\bar{D} \subset G\left(z_{j}\right)$ and thereby the full admissibility condition. We come back to this problem in the second 
part, where we consider different reconstruction schemes for inverse obstacle reconstruction problems.

Recall that the fundamental solution $\Phi$ for both partial differential equations is a function of the distance of its arguments. Hence we see that

$$
\Phi\left(M\left(z_{j}\right) x+z_{j}, z_{j}\right)=\Phi(x, 0)
$$

holds for all $x \in \mathbb{R}^{m}$. In particular, two corresponding points $x_{j} \in G\left(z_{j}\right)$ and $x_{k} \in G\left(z_{k}\right)$ admit the same value of the corresponding right hand side, more precisely for $x_{k}=M\left(z_{k}\right) x_{0}+z_{k}$ and $x_{j}=M\left(z_{j}\right) x_{0}+z_{j}$ with $x_{0} \in G_{0}$ there holds $\Phi\left(x_{j}, z_{j}\right)=\Phi\left(x_{k}, z_{k}\right)$. Moreover, applying the orthogonal translation

$$
x \mapsto M(z) x+z
$$

to the argument of a Herglotz wave function results again in a Herglotz wave function with a modified density due to the functional equation of the exponential function. We can make use of these facts to prove that in the case of congruent configurations $\mathcal{F}_{j}$, it is sufficient to solve only one ill-posed integral equation for the construction of the approximating functions $v_{z_{j}}$. For example, it is sufficient to solve the regularized equation (5.89) for the point $z=0$, and then calculate all $v_{z_{j}}$ by a multiplication of the density $g_{0, \varepsilon}$ which was pointed out in [57] by Potthast. We summarize this property in the following theorem for the Helmholtz equation.

Theorem 5.12. Let $\varepsilon>0,0 \notin G_{0}$ and $g_{0, \varepsilon} \in L^{2}\left(\mathbb{S}^{m-1}\right)$ be the solution to the regularized integral equation

$$
\left(\alpha I+H^{*} H\right) g=H^{*} \Phi(\cdot, 0)
$$

on $\partial G_{0}$ such that

$$
\left\|H g_{0, \varepsilon}-\Phi(\cdot, 0)\right\|_{L^{2}\left(\partial G_{0}\right)}<\varepsilon .
$$

Then, for any $z \in \mathcal{S}$ with corresponding approximation domain $G(z)$ of the form (5.86) the density

$$
g_{z, \varepsilon}(d)=e^{-i \kappa z \cdot d} g_{0, \varepsilon}\left(M(z)^{t} d\right)
$$

defines a Herglotz wave function with

$$
\left\|H g_{z, \varepsilon}-\Phi(\cdot, z)\right\|_{L^{2}(\partial G(z))}<\varepsilon .
$$

Proof. We define

$$
G^{\prime}(z):=\left\{M(z) x: x \in G_{0}\right\} .
$$

Then, we obtain for the left-hand side of (5.92) with fixed $z \in \mathcal{S}$

$$
\begin{aligned}
& \left\|\int_{\mathbb{S}^{m-1}} e^{i \kappa x \cdot d} g_{z, \varepsilon}(d) d s(d)-\Phi(x, z)\right\|_{L^{2}(\partial G(z))} \\
& =\left\|\int_{\mathbb{S}^{m-1}} e^{i \kappa(x+z) \cdot d} g_{z, \varepsilon}(d) d s(d)-\Phi(x+z, z)\right\|_{L^{2}\left(\partial G^{\prime}(z)\right)} \\
& =\left\|\int_{\mathbb{S}^{m-1}} e^{i \kappa z \cdot d} e^{i \kappa x \cdot d} g_{z, \varepsilon}(d) d s(d)-\Phi(x, 0)\right\|_{L^{2}\left(\partial G^{\prime}(z)\right)} .
\end{aligned}
$$


With $\operatorname{det}(M(z))=1$ and $\Phi(M(z) x, 0)=\Phi(x, 0)$ the last equation simplifies to

$$
\begin{aligned}
& \left\|\int_{\mathbb{S}^{m-1}} e^{i \kappa z \cdot d} e^{i \kappa(M(z) x) \cdot d} g_{z, \varepsilon}(d) d s(d)-\Phi(x, 0)\right\|_{L^{2}\left(\partial G_{0}\right)} \\
& =\left\|\int_{\mathbb{S}^{m-1}} e^{i \kappa z \cdot d} e^{i \kappa x \cdot\left(M(z)^{t} d\right)} g_{z, \varepsilon}(d) d s(d)-\Phi(x, 0)\right\|_{L^{2}\left(\partial G_{0}\right)} \\
& =\left\|\int_{\mathbb{S}^{m-1}} e^{i \kappa z \cdot(M(z) \tilde{d})} e^{i \kappa x \cdot \tilde{d}} g_{z, \varepsilon}(M(z) \tilde{d}) d s(\tilde{d})-\Phi(x, 0)\right\|_{L^{2}\left(\partial G_{0}\right)}
\end{aligned}
$$

Inserting the definition of $g_{z, \varepsilon}$ into equation (5.95) ends the proof.

With the previous theorem we can reduce the complexity of calculating the densities $g_{z_{j}, \varepsilon}$ from solving $N$ integral equations of the second kind to the computational cost of solving the integral equation (5.89) once and performing the multiplication (5.91) of the reference density $N-1$ times.

We can transfer this proof easily to the static case when we both replace the Herglotz wave operator with its static analogon and use the static Herglotz wave functions

$$
v_{z}(x)=\operatorname{Re} \int_{\mathbb{S}^{1}} e^{i\left(i d+d^{\perp}\right) \cdot x} g_{z}(d) d s(d), \quad x \in \mathbb{R}^{2}
$$

for the point source approximation.

Theorem 5.13. Let $\varepsilon>0,0 \notin G_{0}$ and $g_{0, \varepsilon} \in L^{2}\left(\mathbb{S}^{1}\right)$ be the solution to the regularized integral equation

$$
\left(\alpha I+H^{*} H\right) g=H^{*} \Phi(\cdot, 0)
$$

on $\partial G_{0}$ such that

$$
\left\|H g_{0, \varepsilon}-\Phi(\cdot, 0)\right\|_{L^{2}\left(\partial G_{0}\right)}<\varepsilon .
$$

Then, for any $z \in \mathcal{S}$ with corresponding approximation domain $G(z)$ of the form (5.86) the density

$$
g_{z, \varepsilon}(d)=e^{-i\left(i d+d^{\perp}\right) \cdot z} g_{0, \varepsilon}\left(M(z)^{t} d\right)
$$

defines a static Herglotz wave function

$$
v_{z, \varepsilon}(x)=\operatorname{Re} \int_{\mathbb{S}^{1}} e^{i\left(i d+d^{\perp}\right) \cdot x} g_{z, \varepsilon}(d) d s(d), \quad x \in \mathbb{R}^{2}
$$

with

$$
\left\|v_{z, \varepsilon}-\Phi(\cdot, z)\right\|_{L^{2}(\partial G(z))}<\varepsilon
$$


We demonstrate the numerical implementation of formulae (5.91) and (5.101) for a circular approximation domain

$$
G_{0}:=\{(x, y)=(r+\delta, 0)+(r \cos t, r \sin t): 0 \leq t \leq 2 \pi\}
$$

of radius $r>0$ and with distance $\delta>0$ from the source point $z=0$. For the approximation domains we choose the strategy

$$
\mathcal{G}=\left\{G(z)=M(z) G_{0}+z: z \in \mathcal{S}\right\}
$$

with the rotation matrix $M(z)=M(\phi)$ given by

$$
M(\phi)=\left(\begin{array}{cc}
\cos (\phi+\pi) & -\sin (\phi+\pi) \\
\sin (\phi+\pi) & \cos (\phi+\pi)
\end{array}\right)=\left(\begin{array}{cc}
-\cos \phi & \sin \phi \\
-\sin \phi & -\cos \phi
\end{array}\right)
$$

and the angle $\phi$ defined via the polar representation $z=\varrho e^{i \phi}$ and $0 \leq$ $\phi<2 \pi$. This strategy moves the fixed configuration $\left(0, G_{0}\right)$ through the domain $\Omega$ of interest in the following way. For each $z \in \Omega$ the center of the corresponding approximation domain $G(z)$ is the point on the ray starting in $z$ and passing through 0 which has distance $r+\delta$ from $z$. This strategy can be applied in the situation where we search for an unknown obstacle $D$ when some a priori information about its size and location is available, since this strategy "looks for" domains near the origin with diameter less than $2 r$.

We first calculate the density $g_{0, \varepsilon}$ by solving the regularized equation (5.89) for Helmholtz' or (5.97) for Laplace's equation for some small regularization parameter $\alpha>0$. In principle, one can determine $\alpha$ by solving

$$
F(\alpha)=\left\|H g_{\alpha}-\Phi(\cdot, 0)\right\|_{L^{2}\left(\partial G_{0}\right)}^{2}-\varepsilon^{2}=0,
$$

where $g_{\alpha}$ denotes the unique solution of (5.89) with Newton's method. However, we avoid to involve this machinery and choose $\alpha$ by trial and error. This is justified since we neither have to deal with noise on the known function $\Phi(\cdot, 0)$ nor do we have to solve the regularized equation several times. For the numerical implementation we discretize both the unit sphere $\mathbb{S}^{1}$ and the boundary $\partial G_{0}$ of the reference approximation domain and apply Nyström's method to solve the regularized integral equation of the second kind. In particular we use a uniform discretization

$$
\mathcal{P}=\left\{d_{k}=\left(\cos t_{k}, \sin t_{k}\right): t_{k}=\frac{k \pi}{n}, \quad k=0,1, \ldots, 2 n-1\right\}
$$

of the unit sphere, which we need in view of (5.91) and (5.99) to ensure rotational invariance of the point set $\mathcal{P}$ under rotations with an angle of $\theta_{k}=k \pi / n$ for $k=0,1, \ldots, 2 n-1$. Consequently, we can not allow arbitrary rotations $M(z)$ of the reference configuration and we require the rotation matrix $M(z)$ to be in the set 


$$
\mathcal{M}=\left\{M\left(\theta_{k}\right): k=0,1, \ldots, 2 n-1\right\} .
$$

Therefore, either the set of sampling points $\mathcal{S}$ has to form a subset of a grid based on polar-coordinates with the angles $\theta_{k}$, i.e. $\mathcal{S}$ has to fulfill

$$
\mathcal{S} \subset\left\{\left(\varrho \cos \theta_{k}, \varrho \sin \theta_{k}\right): \varrho>0, \quad k=0,1, \ldots, 2 n-1\right\},
$$

or we have to choose a different matrix-valued function $M: \mathcal{S} \rightarrow \mathrm{SO}(2, \mathbb{R})$. In the latter case one can use the modified strategy

$$
\tilde{\mathcal{G}}=\left\{G(z)=\tilde{M}(z) G_{0}+z: z \in \mathcal{S}\right\}
$$

with the rotation matrices $\tilde{M}(z)=M(\theta)$ defined by the angle $\theta=\theta_{k}$ such that $\theta_{k}$ minimizes $\left|\phi-\theta_{k}\right|$ with $z=\varrho e^{i \phi}$ or equivalently such that the corresponding unit vector $d_{k}$ maximizes the scalar product

$$
\frac{z}{|z|} \cdot d_{k}, \quad z \neq 0
$$

For sampling points $z$ which lie in the set

$$
\left\{\left(\varrho \cos \frac{2 m-1}{2} \frac{\pi}{n}, \varrho \sin \frac{2 m-1}{2} \frac{\pi}{n}\right): \varrho>0, \quad m=1,2, \ldots, 2 n\right\}
$$

there are two minimizing angles in $[0,2 \pi)$. Therefore we always choose the smallest minimizing angle to avoid any ambiguity in the definition of the function $M$. We remark that for a set of sampling points $\mathcal{S}$ with the property (5.108) we have $\mathcal{G}=\tilde{\mathcal{G}}$.

In either case we can calculate the density $g_{z, \varepsilon}$ corresponding to the approximation domain $G(z) \in \tilde{\mathcal{G}}$ and the source point $z=\varrho e^{i \phi}$ on the point set $\mathcal{P}$ by

$$
g_{z, \varepsilon}\left(d_{k}\right)=h_{z}\left(d_{k}\right) g_{0, \varepsilon}\left(d_{j}\right)
$$

with $j=k-l \bmod 2 n$ and where $l$ is chosen such that $d_{l}$ minimizes $\left|\phi-d_{l}\right|$. The function $h_{z}$ is given by

$$
h_{z}(d)=e^{-i\left(i d+d^{\perp}\right) \cdot z}, \quad d \in \mathbb{S}^{1}
$$

for the Laplace equation and

$$
h_{z}(d)=e^{-i \kappa d \cdot z}, \quad d \in \mathbb{S}^{1}
$$

for the Helmholtz equation.

We conclude this chapter with a short comment on the numerical implementation of the preceeding algorithm in three dimensions. The requirement of a uniform discretization in the sense that its point set $\mathcal{P}$ is invariant under as many rotations as possible, admits only special geometrical configurations. Either such a point set arises from one of the platonic bodies 
or all rotations that fix the point set have one rotation axis in common. While the platonic bodies lead to a balanced distribution of rotation axis the second kind of point set is restricted to one rotation axis. Hence for a balanced distribution of rotation axis we have to choose several such point sets with different rotation axis. On the other hand this point set yields a straightforward generalization of the twodimensional algorithm whereas the numerical implementation of a threedimensional algorithm based on the platonic bodies seems to be unmanageable. Either case has a catch in it and is beyond the main focus of this work. Therefore we drop the requirement of a uniform discretization of the unit sphere and consider translations of the reference configuration only. Then the strategies for the approximation domains are of the form

$$
\mathcal{G}=\left\{G(z)=G_{0}+z: z \in \mathcal{S}\right\}
$$

and lead to a simple pointwise function multiplication without involving rotations of the argument when calculating the densities $g_{z, \varepsilon}$ for the translated approximation domains. 

Part III

Reconstruction Schemes 



\section{The Point Source Method}

The point source method (PSM) as proposed by Potthast in a series of papers [52], [53], [56] and in his monograph [54] is a scheme for the reconstruction of scattered acoustic or electromagnetic fields from their far field pattern. It belongs to the class of decomposition methods in inverse scattering, see [57], since it solves the nonlinear and ill-posed inverse shape reconstruction problem by a decomposition into a linear ill-posed problem and a nonlinear well-posed problem. In the first ill-posed step the PSM reconstructs the scattered field from the far field pattern with a linear backprojection operator. With this knowledge the PSM solves the nonlinear but well-posed problem to find the zeros of the total field (Dirichlet boundary condition) or its normal derivative (Neumann boundary condition) in a second step. Hence the point source method can also be regarded as an analytic continuation method extending the far field of a scattered wave to its near field.

In [14], Potthast and the author presented a redesign of the point source method for the application to boundary value problems where the total field $u$ together with its normal derivative is given on a measurement boundary instead of information about the asymptotical behaviour of the scattered field at infinity. The redesign of the point source method was based on Green's theorem and constructed the backprojection operator without using the reciprocity relation. This approach has been first suggested by Liu et al. [5] as an alternative proof for the point source method in the range of scattering problems.

The redesign of the point source method for boundary value problems was developed using a single-layer potential on the measurement boundary for the approximation of the point source rather than a Herglotz wave function. Hence, the strategy for calculating the approximating functions efficiently by a simple function multiplication with an argument shift is not applicable in this case. We will remedy this drawback of the PSM for boundary value problems in this work and present a reconstruction scheme that approximates the point source with a Herglotz wave function and which therefore leads to a fast numerical implementation of the PSM algorithm. 
In this chapter we first investigate the PSM for boundary value problems which can be seen as the natural setting for the inverse scattering problem. In this situation we do not measure the far field pattern which describes the asymptotical behaviour of the scattered field far away from the obstacle, but we measure the Cauchy data of the total field on a measurement boundary $\partial \Omega$ of a domain $\Omega$ containing the scatterer. Assuming a perfect measurement device we would be able to measure the Cauchy data on $\partial \Omega$ exactly while any physical measurement of the far field pattern always has to deal with an approximation error since the far field pattern can only be deduced from a measurement of the scattered field in a finite distance. Therefore we avoid any approximation error by considering the scattered field in finite distance from the scattering obstacle as the input data for the reconstruction scheme. Of course, in practice the approximation error can usually be neglected in view of the error of the underlying measurement device. Nevertheless, a reformulation of the point source method for near field measurements will give new and deeper insight into this method.

Although we may ignore the approximation error due to measurements in a finite distance there is no way to measure the scattered field itself since it will sum up together with the incident field to the total field due to the principle of superposition. Therefore we can not measure the scattered field directly. Hence, the calculation of the far field pattern from measurements of the total field at what we consider to be infinity needs the knowledge of the incident field, too. Again, in any practical application we are facing an idealization concerning the geometrical form of the incident wave. To illustrate this argument let us consider an incident plane wave for example. We can generate an approximation of a plane wave by a harmonic oscillation of a large but finite plate or membrane. But the more we move away from the center of the plate to the edge of the cone of sound the more the geometrical form of the emitted wave will differ from that of an ideal plane wave. However, the wave number of the emitted and the ideal plane wave will be the same as long as we assume a machine that can generate an exact oscillation.

The point source method as we will present it now for the inverse scattering boundary value problem does not make any use of the form of the incident wave and only assumes the knowledge of its wave number. Hence we regard the PSM for the inverse scattering boundary value problem a very natural and practical approach to inverse scattering problems, especially in applications where the far field pattern is not available or the type of incident wave is not known.

We illustrate the flexibility of the PSM by applying it both to an inverse boundary value problem in acoustics and in electrostatics before we turn our interest to the inverse acoustic scattering problem. We demonstrate that the PSM for inverse scattering arises from the PSM for boundary value problems by considering large measurement boundaries and letting tend the 
radius of these measurement boundaries to infinity. From this point of view we get a new insight of the PSM as introduced by Potthast for the inverse scattering case. In particular we do not use the mixed reciprocity relation, see Theorem 2.2.4 in [54], in the derivation of the point source method. In consequence, we can apply the point source method to inverse scattering and boundary value problems with arbitrary incident fields. Furthermore we provide a new characterization of the enlighted area in terms of an indicator function which relates the point source method with the no response test, see [43] and [58].

In the last section of this chapter we present numerical examples for the (sound-soft) obstacle reconstruction problem of both the inverse boundary value and the inverse acoustic scattering problem as well as for the inverse EIT problem.

\subsection{The PSM for Boundary Value Problems}

The point source method was first introduced by Potthast in [52] in the year 1996 and redesigned for the application to inverse boundary value problems in [14] seven years later. Here, we will present this redesign in a more general way and emphasize the connections to the original PSM. Let us consider the following inverse boundary value problems.

Acoustic scattering. We recall the formulation of the direct problem first. For given data $f \in C(\partial \Omega)$ we find a field $u$ which satisfies

$$
\begin{aligned}
\Delta u+\kappa^{2} u=0 & \text { in } \Omega \backslash \bar{D}, \\
u=0 & \text { on } \partial D, \\
u=f & \text { on } \partial \Omega,
\end{aligned}
$$

where $\operatorname{Re}(\kappa)>0$ and $\operatorname{Im}(\kappa) \geq 0$. We investigated the direct problem in Section 4.2 and proved existence as well as uniqueness and presented a numerical scheme for the calculation of the solution to this problem.

Given $u$ and $\frac{\partial u}{\partial \nu}$ on $\partial \Omega$, where $\nu$ denotes the exterior unit normal on $\partial \Omega$, the inverse problem is to reconstruct $u$ in $\Omega \backslash D$ and to find the boundary $\partial D$ of the unknown obstacle. By the boundary condition (6.2) $u$ vanishes on the boundary $\partial D$ of a sound-soft obstacle. From the field $u$ we will reconstruct the set of zeros of $u$ and consider it as our solution $\partial D_{\text {rec }}$ to the domain reconstruction problem.

Electrical impedance tomography. As a second problem we consider a special case of electrical impedance tomography, where we search for a perfectly conducting inclusion in a homogeneous background medium. Now, the direct problem is to find a solution to the problem

$$
\begin{aligned}
\Delta u=0 & \text { in } \Omega \backslash \bar{D}, \\
u=0 & \text { on } \partial D, \\
u=f & \text { on } \partial \Omega,
\end{aligned}
$$


for a continuous function $f \in C(\partial \Omega)$. For the solution theory for this problem we refer to Section 4.2 of this work.

Again, given $u$ and $\frac{\partial u}{\partial \nu}$ on $\partial \Omega$, the inverse problem is to reconstruct $u$ in $\Omega \backslash D$ and to find the boundary $\partial D$ of the obstacle.

We remark that the inverse acoustic boundary value problem is not unique, i.e. there might be two different obstacles $D_{1}$ and $D_{2}$ admitting solutions $u_{1}, u_{2}$ to the corresponding direct boundary value problems such that their Cauchy data coincide on the measurement boundary $\partial \Omega$.

Example 6.1 (Non-Uniqueness of the inverse BVP for Helmholtz' equation). Consider the domain $\Omega=B_{R} \subset \mathbb{R}^{3}$ with $R>2 \pi$ and the boundary value problem $(6.1)-(6.3)$ with wave number $\kappa=1$ for the obstacle $D_{1}=B_{\pi}$ and $D_{2}=B_{2 \pi}$, respectively. If the radius $R$ of the measurement boundary is chosen such that $\kappa=1$ is neither a Dirichlet eigenvalue of the negative Laplacian in $\Omega \backslash \bar{D}_{1}$ nor in $\Omega \backslash \bar{D}_{2}$ then both boundary value problems are uniquely solvable.

In this situation, we could not decide whether the measurement of the Cauchy data

$$
u(x)=\frac{\sin |x|}{|x|}=\frac{\sin R}{R}, \quad \frac{\partial u}{\partial \nu}(x)=\frac{\partial}{\partial \nu} \frac{\sin |x|}{|x|}=\frac{\cos R}{R}-\frac{\sin R}{R^{2}}
$$

on $\partial \Omega$ is produced by the sound-soft obstacle $D_{1}$ or by the sound-soft obstacle $D_{2}$ since

$$
u(x)=\frac{\sin |x|}{|x|}, \quad x \neq 0,
$$

solves the direct boundary value problem (6.1) - (6.3) for both obstacles $D_{1}$ and $D_{2}$ with the Cauchy data (6.7).

We will see in the next section that Example 6.1 carries over to an example of nonuniqueness in the inverse scattering problem. Indeed, Example 6.1 highlights one of the characteristic situations when nonuniqueness can occur in the inverse boundary value problem. For a uniqueness proof of the inverse boundary value problem with a finite set of Cauchy data on the measurement boundary the following variant of Holmgren's theorem is crucial.

Theorem 6.2 (Holmgren's Theorem). Let $G$ be a bounded and connected domain with boundary of class $C^{2}$ and let $u \in C^{2}(G) \cap C^{1}(\bar{G})$ be a solution to the Helmholtz equation

$$
\Delta u+\kappa^{2} u=0 \quad \text { in } G,
$$

with

$$
u=\frac{\partial u}{\partial \nu}=0
$$

on a nonempty open subset $\Gamma \subset \partial G$. Then $u=0$ in $G$. 
For the proof of Holmgren's theorem we adapt Theorem 4.1.2.4 in [37].

Proof. For $x \in \mathbb{R}^{m} \backslash \bar{G}$ the fundamental solution $\Phi(x, \cdot)$ solves the Helmholtz equation in $G$. Thus

$$
0=\int_{\partial G}\left(\Phi(x, y) \frac{\partial u}{\partial \nu}(y)-\frac{\partial \Phi(x, y)}{\partial \nu(y)} u(y)\right) d s(y)
$$

by (2.5) and since the Cauchy data of $u$ vanishes on $\Gamma$ we obtain

$$
v(x)=\int_{\partial G \backslash \Gamma}\left(\Phi(x, y) \frac{\partial u}{\partial \nu}(y)-\frac{\partial \Phi(x, y)}{\partial \nu(y)} u(y)\right) d s(y)=0
$$

for all $x \in \mathbb{R}^{m} \backslash \bar{G}$. Now, $v$ is a solution to the Helmholtz equation in $\Gamma \cup\left(\mathbb{R}^{m} \backslash \partial G\right)$ and we can extend this function analytically into $G$. Hence $v=0$ in $G$ and after adding the vanishing integral over the set $\Gamma$ we finally deduce $u=0$ in $G$ from Green's representation theorem.

Indeed Holmgren's theorem can be generalized for a larger class of partial differential equations, see [73]. Now we can characterize the situations in which nonuniqueness can occur for one set of Cauchy data in more detail and prove uniqueness for the case of a finite number of measurements of linear independent Cauchy data on $\partial \Omega$.

Theorem 6.3. If two sound-soft obstacles $D_{1}, D_{2} \subset \mathbb{R}^{3}$ admit the same set of Cauchy data

$$
u=f \neq 0, \quad \frac{\partial u}{\partial \nu}=g
$$

on $\partial \Omega$, then both $D_{1} \backslash D_{2}$ and $D_{2} \backslash D_{1}$ is either the empty set or $-\kappa^{2}$ is a Dirichlet-eigenvalue of the Laplacian in this domain.

Furthermore, assume that the obstacle $D$ is sound-soft and contained in a ball of radius $R>0$ centered at the origin. Let $t_{n l}, l \in \mathbb{N}$ be the positive zeros of the spherical Bessel function $j_{n}$ of order $n$ and define

$$
N:=\sum_{t_{n l}<\kappa R}(2 n+1) \text {. }
$$

Then the boundary $\partial D$ is uniquely determined by the measurement of $N+1$ linear independent sets of Cauchy data $\left(f^{(1)}, g^{(1)}\right), \ldots,\left(f^{(N+1)}, g^{(N+1)}\right)$ on $\partial \Omega$ with $f^{(j)} \neq 0$ for $1 \leq j \leq N+1$.

The proof follows the ideas of the uniqueness proof of Colton and Sleeman given in [9] for the inverse scattering problem. An improvement on the number $N$ of linear independent Cauchy data can be obtained with a uniqueness result of Gintides, see [18].

Proof. Let $u_{i}$ solve the Helmholtz equation in $\Omega \backslash \bar{D}_{i}$ with the Cauchy data

$$
u_{i}=f \neq 0, \quad \frac{\partial u_{i}}{\partial \nu}=g
$$


on $\partial \Omega$ for $i=1,2$. In view of Holmgren's theorem the function $u=u_{1}-u_{2}$ has to vanish in $\mathbb{R}^{3} \backslash\left(D_{1} \cup D_{2}\right)$. Let $G=D_{2} \backslash \bar{D}_{1}$ be a nonempty set. Then $u_{1}=0$ on $\partial G \cap \partial D_{1}$ and also $u_{1}=u_{2}=0$ on $\partial G \cap \partial D_{2}$ due to the soundsoft boundary condition of the obstacles. Therefore $u_{1}$ solves the Helmholtz equation in $G$ with homogeneous boundary data $u_{1}=0$ on $\partial G$. Either $u_{1}$ is zero in $G$ or an eigenfunction to the eigenvalue $-\kappa^{2}$ of the Laplacian in the domain $G$. But in the first case an analytic continuation argument shows $u_{1}=0$ in $\mathbb{R}^{3} \backslash \bar{D}_{1}$ which contradicts $u_{1}=f \neq 0$ on $\partial \Omega$. Hence we have proven the first statement of the theorem.

For the second statement we use a corresponding notation and assume that $u_{1}^{(j)}$ are eigenfunctions to the eigenvalue $\kappa^{2}$ of $-\Delta$ in $G$ for $1 \leq j \leq N+1$. Otherwise, by the first part of the proof there exists some $k$ with $1 \leq k \leq$ $N+1$ such that $u_{1}^{(k)}=0$ in $\mathrm{G}$, which contradicts $f^{(k)} \neq 0$. Moreover the $N+1$ eigenfunctions $u_{1}^{(j)}$ are linear independent due to our assumption on the Cauchy data.

In the following we prove that the dimensionality of the space of eigenfunctions of the negative Laplacian in the domain $G$ is less than $N+1$ which contradicts the previous statement and ends the proof. To this end we proceed as in the proof of Colton and Sleeman presented in [9] and reformulated in [7]. We denote with $\lambda_{1} \leq \ldots \leq \lambda_{m}=\kappa^{2}$ the eigenvalues of the negative Laplacian in $G_{0}$ that are less than or equal to $\kappa^{2}$ counted with respect to their multiplicity. Furthermore let $\mu_{1} \leq \ldots \leq \pm \mu_{m}$ be the first $m$ eigenvalues of $-\Delta$ in the ball of radius $R$. Then we obtain $\mu_{m} \leq \lambda_{m}=\kappa^{2}$ since the $n$-th eigenvalue of a domain is always smaller or equal than the $n$-th eigenvalue of any of its subdomains. We can conclude that the multiplicity of $\lambda_{m}$ is less than or equal to the smallest integer $m_{0}$ such that $\mu_{m_{0}} \geq \kappa^{2}$. In particular the eigenfunctions of a ball of radius $R$ are the spherical wave functions $j_{n}\left(\sqrt{\mu_{n l}}|x|\right) Y_{n}(\hat{x})$ corresponding to the eigenvalues $\mu_{n l}=t_{n l}^{2} / R^{2}$, where each eigenvalue $\mu_{n l}$ has the multiplicity $2 n+1$, see Theorem 2.6 in [7]. Hence we can estimate $m_{0} \leq N$ where $N$ is the number defined in the theorem and finish the proof.

For small domains $\Omega$ of interest we even have uniqueness for one measurement of the Cauchy data.

Corollary 6.4. Let $\Omega$ be contained in a ball of radius $R$ such that $\kappa R<\pi$. If the Cauchy data

$$
u_{i}=f \neq 0, \quad \frac{\partial u_{i}}{\partial \nu}=g
$$

of two sound-soft scatterers $D_{1}, D_{2} \subset \Omega$ coincide on $\partial \Omega$ then $D_{1}=D_{2}$.

The proof of this corollary is a simple reformulation of the proof of Corollary 5.3 in [7]. Theorem 6.3 and Corollary 6.4 can be carried over to the two-dimensional boundary value problem in a straightforward way if we replace the spherical bessel functions $j_{n}$ with the ordinary Bessel functions $J_{n}$. Furthermore, we have to use the fact that there exist only two linearly 
independent spherical harmonics in $\mathbb{R}^{2}$, which affects the constant (6.14). Obviously, we also have to replace the smallest positive zero $t_{01}=\pi$ of the spherical bessel function with the smallest positive zero $t_{01} \approx 2.4048$ of the ordinary bessel function in Corollary 6.4.

In the static case $\kappa=0$ we obtain uniqueness of the inverse boundary value problem for electrostatics with Holmgren's theorem.

Theorem 6.5 (Holmgren's Theorem). Let $G$ be a bounded and connected domain with boundary of class $C^{2}$ and let $u \in C^{2}(G) \cap C^{1}(\bar{G})$ be a solution to the Laplace equation

$$
\Delta u=0 \quad \text { in } G \text {, }
$$

with

$$
u=\frac{\partial u}{\partial \nu}=0
$$

on a nonempty open subset $\Gamma \subset \partial G$. Then $u=0$ in $G$.

The proof of Holmgren's theorem for the Laplace equation follows the same arguments as before. With Holmgren's theorem we can prove the uniqueness of the inverse boundary value problem in electrical impedance tomography.

Theorem 6.6. Let $D_{1}$ and $D_{2}$ be two bounded and perfectly conducting subdomains of the bounded domain $\Omega \subset \mathbb{R}^{m}, m=2,3$. Let $u_{1}, u_{2}$ be the solutions to the corresponding direct boundary value problems (6.4) - (6.6). If the Cauchy data on $\partial \Omega$ coincide, i.e.

$$
u_{1}=u_{2}=f \neq 0, \quad \frac{\partial u_{1}}{\partial \nu}=\frac{\partial u_{2}}{\partial \nu}
$$

on $\partial \Omega$, then $D_{1}=D_{2}$.

Proof. Suppose $D_{1} \neq D_{2}$ and the Cauchy data (6.19) of the solutions $u_{1}, u_{2}$ to the corresponding boundary value problems coincide. Then $u_{1}=u_{2}$ in $\Omega \backslash\left(D_{1} \cup D_{2}\right)$ by Holmgren's theorem. Without loss of generality we assume $D_{1} \backslash D_{2}$ to be a nonempty set. Hence $u_{2}=u_{1}=0$ on $\partial D_{1} \backslash D_{2}$ and since $u_{2}=0$ on $\partial D_{2} \cap D_{1}$ we obtain $u_{2}=0$ on $\partial\left(D_{1} \backslash D_{2}\right)$. By the minimummaximum principle for harmonic functions $u_{2}$ vanishes first in the nonempty set $D_{1} \backslash D_{2}$ and then by analytic continuation in the whole domain $\Omega \backslash D_{2}$, which contradicts the assumption $u_{2}=f \neq 0$ on $\partial \Omega$.

Let us now return to the task of reconstructing an unknown obstacle $D$ from the Cauchy data of some physical meaningful field $u$ on a measurement boundary $\partial \Omega$. We will deduce the PSM for both the acoustic and the electrostatic case simultaneously. Therefore care has to be taken when interpreting the function $\Phi(x, y)$ since we do not distinguish between the 
fundamental solution of the Laplace and that of the Helmholtz equation in our notation.

In this chapter we will assume the knowledge of some physical property of the unknown obstacle, which has to be of sound-soft or perfectly conducting type respectively. In either case we suppose the physical field $u$ to vanish on the boundary of $D$ and try to reconstruct the obstacle with a decomposition method. In the first step we will reconstruct the field $u$ from its Cauchy data on $\partial \Omega$, i.e. we try to extend $u$ from its values on the boundary to the interior. We mention that in this first step we do not use the boundary condition on $\partial D$. It will not be needed until we are are looking for the boundary $\partial D$ in the second step when we track down the points $z \in \Omega$ in which the reconstructed field matches the boundary condition. We demonstrate this procedure in the case of a Dirichlet boundary condition, where we identify the boundary of the reconstructed obstacle $\partial D_{\text {rec }}$ with the zeros of the reconstructed field.

For the analytic continuation of the given Cauchy data into the domain $\Omega$ we remark that the true field may be extended beyond the domain $\Omega \backslash D$ into the interior of $D$ which is illustrated in Example 6.1. However the extension of the field inside $D$ has no physical meaning. Therefore we call $\Omega \backslash \bar{D}$ the physical domain. Furthermore if there is no a priori information about the position of the obstacle available we have to attempt to extend the field into the interior of $\Omega$ as far as possible. Hence the domain of interest $\mathcal{B}$, where we search for the obstacle and try to reconstruct the field is all of $\Omega$. On the other hand, for applications with some additional information about the obstacle we can make the domain of interest smaller. For example, if we already know that the obstacle is located around the origin and contains the ball $B_{r}, r>0$, then we only need to reconstruct the field in the smaller domain $\mathcal{B}=\Omega \backslash B_{r}$ of interest.

For the analytic continuation of the field $u$ into its physical domain $\Omega \backslash$ $\bar{D}$ we recall the representation Theorems 2.11 and 2.17 for Laplace's and Helmholtz' equation. In our setting the representation formula is given by

$$
\begin{aligned}
u(x)= & \int_{\partial \Omega}\left(\frac{\partial u}{\partial \nu}(y) \Phi(x, y)-\frac{\partial \Phi(x, y)}{\partial \nu(y)} u(y)\right) d s(y) \\
& +\int_{\partial D}\left(\frac{\partial u}{\partial \nu}(y) \Phi(x, y)-\frac{\partial \Phi(x, y)}{\partial \nu(y)} u(y)\right) d s(y)
\end{aligned}
$$

for $x \in \Omega \backslash \bar{D}$, where the unit normal vector $\nu$ points into the exterior of the domain $\Omega \backslash \bar{D}$. In contrast to the presentation of the PSM for boundary value problems in [14] we did not use the boundary condition in (6.20) to emphasize the independence of the first reconstruction step of the physical properties of the obstacle.

The representation theorem sets up a correspondence between the field values inside the physical domain $\Omega \backslash \bar{D}$ and its values on the boundary. Hence 
(6.20) describes the analytic continuation for the field $u$ from its Cauchy data on $\partial(\Omega \backslash \bar{D})$ into the domain $\Omega \backslash \bar{D}$. The first integral on the righthand side of (6.20) will be given by a measurement of $u$ and $\frac{\partial u}{\partial \nu}$ on the boundary $\partial \Omega$. However, the values of the field on $\partial D$ are not available, so the second integral is unknown and we lack some information in this analytic continuation formula, which has to be remedied in the following using the knowledge of $u$ and $\frac{\partial u}{\partial \nu}$ on the boundary $\partial \Omega$ only.

To this end we consider a function $v \in C^{2}(\Omega \backslash \bar{D}) \cap C^{1}(\bar{\Omega} \backslash D)$ that solves the underlying partial differential equation in the physical domain. As a consequence of Green's second formula (2.5) we obtain

$$
\int_{\partial \Omega}\left(v \frac{\partial u}{\partial \nu}-u \frac{\partial v}{\partial \nu}\right) d s+\int_{\partial D}\left(v \frac{\partial u}{\partial \nu}-u \frac{\partial v}{\partial \nu}\right) d s=0
$$

since both functions $u$ and $v$ are solutions to the PDE under consideration in the domain $\Omega \backslash \bar{D}$.

Let us now assume that for any error level $\varepsilon>0$ and every $z$ in some subset $\mathcal{E} \subset \mathcal{B}$ of the domain of interest we can find a solution $v_{z, \varepsilon}$ to the PDE in the domain $\mathcal{B}$ which approximates the point source $\Phi(\cdot, z)$ on $\partial D$ by satisfying

$$
\left\|\Phi(\cdot, z)-v_{z, \varepsilon}\right\|_{C^{1}(\partial D)} \leq \varepsilon .
$$

Then we call the set $\mathcal{E}$ an enlighted area since the points in the set $\mathcal{E}$ admit an approximate analytic continuation formula resulting in an approximation for the field $u$ in $\mathcal{E}$. With the difference function

$$
w_{\varepsilon}(z, y):=\Phi(z, y)-v_{z, \varepsilon}(y), \quad(z, y) \in \mathcal{E} \times \mathcal{B}, \quad z \neq y,
$$

we obtain a very simple definition

$$
u_{\mathrm{rec}, \varepsilon}(z):=\int_{\partial \Omega}\left(\frac{\partial u}{\partial \nu}(y) w_{\varepsilon}(z, y)-\frac{\partial w_{\varepsilon}(z, y)}{\partial \nu(y)} u(y)\right) d s(y), \quad z \in \mathcal{E},
$$

of the reconstructed field and prove its uniform convergence towards the true field in $\mathcal{E}$.

Theorem 6.7. Let $\mathcal{E} \subset \mathcal{B}$ be an enlighted area. Then the reconstructed field $u_{\mathrm{rec}, \varepsilon}$ converges uniformly to the true field on $\mathcal{E}$, i.e.

$$
\lim _{\varepsilon \rightarrow 0} \max _{z \in \mathcal{E}}\left|u(z)-u_{\mathrm{rec}, \varepsilon}(z)\right|=0 .
$$

Proof. With the definition (6.24) of the reconstructed field, the representation formula (6.20) and formula (6.21) applied to $v_{z, \varepsilon}$ and $u$ we derive

$$
\begin{aligned}
\left|u(z)-u_{\mathrm{rec}, \varepsilon}(z)\right|= & \mid \int_{\partial \Omega}\left(\frac{\partial u}{\partial \nu}(y) v_{z, \varepsilon}(y)-\frac{\partial v_{z, \varepsilon}}{\partial \nu(y)} u(y)\right) d s(y) \\
& +\int_{\partial D}\left(\frac{\partial u}{\partial \nu}(y) \Phi(z, y)-\frac{\partial \Phi(z, y)}{\partial \nu(y)} u(y)\right) d s(y) \mid \\
= & \left|\int_{\partial D}\left(\frac{\partial u}{\partial \nu}(y) w_{\varepsilon}(z, y)-\frac{\partial w_{\varepsilon}(z, y)}{\partial \nu(y)} u(y)\right) d s(y)\right|
\end{aligned}
$$


with $w_{\varepsilon}(z, y)$ defined as above. Applying the triangle inequality and the Cauchy-Schwarz inequality we can estimate

$$
\begin{aligned}
\left|u(z)-u_{\mathrm{rec}, \varepsilon}(z)\right| & \leq\left|\int_{\partial D} \frac{\partial u}{\partial \nu}(y) w_{\varepsilon}(z, y) d s(y)\right|+\left|\int_{\partial D} \frac{\partial w_{\varepsilon}(z, y)}{\partial \nu(y)} u(y) d s(y)\right| \\
& \leq c_{1}\left(\int_{\partial D}\left|w_{\varepsilon}(z, y)\right|^{2} d s(y)\right)^{\frac{1}{2}}+c_{2}\left(\int_{\partial D}\left|\frac{\partial w_{\varepsilon}(z, y)}{\partial \nu(y)}\right|^{2} d s(y)\right)^{\frac{1}{2}} \\
& \leq C \varepsilon
\end{aligned}
$$

for all $z \in \mathcal{E}$, where we have used the approximation property (6.22) in the final estimate and where the constants are given by

$$
c_{1}=\left(\int_{\partial D}\left|\frac{\partial u}{\partial \nu}\right|^{2} d s\right)^{\frac{1}{2}}, \quad c_{2}=\left(\int_{\partial D}|u|^{2} d s\right)^{\frac{1}{2}}
$$

and

$$
C=2 \max \left\{c_{1}, c_{2}\right\}\left(\int_{\partial D} 1 d s\right)^{\frac{1}{2}}
$$

We remark that there exists a nontrivial enlighted area if we approximate the point source with a Herglotz wave function (5.44) or a static Herglotz wave function (5.48) on suitable approximation domains. For example, if we approximate the point source on translated approximation domains arising from one fixed reference configuration $\left(0, G_{0}\right)$, where we assume that

$$
\bar{D} \subset z+G_{0}
$$

for at least one $z \in \mathcal{B}$, then $\{z\} \neq \emptyset$ is an enlighted area. More precisely in this case any compact subset $\mathcal{E}$ of the admissibility region

$$
\mathcal{A}:=\{z \in \mathcal{B}: \bar{D} \subset G(z)\}
$$

defines an enlighted area, as we demonstrate in the following. We prove this fact by considering the family of sets

$$
\mathcal{A}_{\varrho}:=\{z \in \mathcal{A}: \operatorname{dist}(\bar{D}, \partial G(z))>\varrho\},
$$

where the distance of the obstacle to the boundary of the translated approximation domain is measured by

$$
\operatorname{dist}(\bar{D}, \partial G(z))=\inf _{(x, y) \in \bar{D} \times \partial G_{0}}|x-(z+y)|
$$

for all $z \in \mathcal{B}$. Notice that $\mathcal{A}_{\varrho}$ is an open set since for any $z \in \mathcal{A}_{\varrho}$ with 


$$
\operatorname{dist}\left(\bar{D}, \partial G(z)=\varrho_{z}>\varrho\right.
$$

it contains the ball $B_{r}(z)$ of radius $r=1 / 2\left(\varrho_{z}-\varrho\right)$ around $z$ and therefore

$$
\bigcup_{\varrho>0} \mathcal{A}_{\varrho} \supset \mathcal{E}
$$

is an open covering of $\mathcal{E}$. Hence there exists a $\varrho_{0}>0$ with $\mathcal{E} \subset \mathcal{A}_{\varrho_{0}}$ due to the compactness of $\mathcal{E}$ and the monotonicity of the sets $\mathcal{A}_{\varrho}$. Following the proof of Lemma 5.6 we see that the the Herglotz wave functions (5.44) with densities

$$
g_{z, \varepsilon}=\left(\alpha I+H^{*} H\right)^{-1} H^{*} \Phi(\cdot, 0)
$$

converge to the fundamental solution $\Phi(\cdot, 0)$ in $L^{2}\left(\partial G_{0}\right)$ as $\alpha=\alpha(\varepsilon)$ tends to zero. Now we consider the compact set

$$
\tilde{G}_{0}:=\left\{x \in G_{0}: \operatorname{dist}\left(x, \partial G_{0}\right) \geq \varrho_{0}\right\}
$$

and apply the second part of Theorem [5.7, which provides the uniform convergence of the Herglotz wave functions towards the fundamental solution $\Phi(\cdot, 0)$ in $\tilde{G}_{0}$ and we may assume

$$
\left\|v_{0, \varepsilon}-\Phi(\cdot, 0)\right\|_{C^{1}\left(\tilde{G}_{0}\right)} \leq \varepsilon .
$$

By Theorem 5.12 we obtain the approximation

$$
\left\|v_{z, \varepsilon}-\Phi(\cdot, z)\right\|_{C^{1}\left(\tilde{G}_{z}\right)} \leq \varepsilon
$$

for the shifted configuration with $\tilde{G}_{z}=z+\tilde{G}_{0}$ and since $\partial D \subset \tilde{G}_{z}$ for all $z \in \mathcal{E}$ we have established the approximation (6.22). This proves that $\mathcal{E}$ is an enlighted area for the Helmholtz equation. For the static case this statement follows from Theorem 5.13 and Lemma 5.3. For a generalization of this result to continuously varying approximation domains we refer to [14].

For the Helmholtz equation we deduce an explicit formula for $u_{\mathrm{rec}, \varepsilon}$ by inserting the approximating Herglotz wave function

$$
v_{z, \varepsilon}(y)=\int_{\mathbb{S}^{m-1}} e^{i \kappa y \cdot d} g_{z, \varepsilon}(d) d s(d), \quad y \in \Omega
$$

and its normal derivative

$$
\frac{\partial v_{z, \varepsilon}}{\partial \nu}(y)=\int_{\mathbb{S}^{m-1}} i \kappa d \cdot \nu(y) e^{i \kappa y \cdot d} g_{z, \varepsilon}(d) d s(d), \quad y \in \Omega
$$

into formula (6.24). This yields 


$$
\begin{aligned}
u_{\mathrm{rec}, \varepsilon}(z)= & \int_{\partial \Omega}\left(\frac{\partial u}{\partial \nu}(y) \Phi(z, y)-\frac{\partial \Phi(z, y)}{\partial \nu(y)} u(y)\right) d s(y) \\
& -\int_{\partial \Omega} \int_{\mathbb{S}^{m-1}} e^{i \kappa y \cdot d} g_{z, \varepsilon}(d)\left(\frac{\partial u}{\partial \nu}(y)-i \kappa d \cdot \nu(y) u(y)\right) d s(d) d s(y)
\end{aligned}
$$

for $z \in \mathcal{E}$.

In case of orthogonal translations of a fixed reference domain we can ascribe the density $g_{z}$ in (6.42) corresponding to a rotated and translated approximation domain $G(z)$ to the original density $g_{0, \varepsilon}$ of the starting domain $G_{0}$ using (5.91) and so we finally obtain

$$
\begin{aligned}
u_{\mathrm{rec}, \varepsilon}(z)= & \int_{\partial \Omega}\left(\frac{\partial u}{\partial \nu}(y) \Phi(z, y)-\frac{\partial \Phi(z, y)}{\partial \nu(y)} u(y)\right) d s(y) \\
& -\int_{\partial \Omega} \int_{\mathbb{S}^{m-1}} e^{i \kappa(y-z) \cdot d} g_{0, \varepsilon}\left(M(z)^{t} d\right) \\
& \left(\frac{\partial u}{\partial \nu}(y)-i \kappa d \cdot \nu(y) u(y)\right) d s(d) d s(y)
\end{aligned}
$$

for $z \in \mathcal{E}$.

In the static case we use the approximating functions

$$
v_{z, \varepsilon}(y)=\operatorname{Re} \int_{\mathbb{S}^{m-1}} e^{i\left(i d+d^{\perp}\right) \cdot y} g_{z, \varepsilon}(d) d s(d), \quad y \in \Omega
$$

and deduce the reconstruction formula

$$
\begin{aligned}
u_{\mathrm{rec}, \varepsilon}(z)= & \int_{\partial \Omega}\left(\frac{\partial u}{\partial \nu}(y) \Phi(z, y)-\frac{\partial \Phi(z, y)}{\partial \nu(y)} u(y)\right) d s(y) \\
& -\operatorname{Re} \int_{\partial \Omega} \int_{\mathbb{S}^{m-1}} e^{i\left(i d+d^{\perp}\right) \cdot(y-z)} g_{0, \varepsilon}\left(M(z)^{t} d\right) \\
& \left(\frac{\partial u}{\partial \nu}(y)-i\left(i d+d^{\perp}\right) \cdot \nu(y) u(y)\right) d s(d) d s(y)
\end{aligned}
$$

for $z \in \mathcal{E}$ in an analogous manner.

But still we lack a characterization for the admissibility region since we can not decide whether $\bar{D} \subset G(z)$ without the knowledge of the unknown obstacle $D$ itself. To this end we present an indicator function for the admissibility region based on the no response test, see [43] and [58], and the approximation properties of the Herglotz wave functions. The no response test calculates the response of some special incident fields $v$ that are chosen such that the impulse size condition

$$
\|v\|_{C^{1}(\bar{G})} \leq \varepsilon
$$

is satisfied on some test domain $G \subset \Omega$ with some small threshold value $\varepsilon>0$. Now, the no response test defines an indicator function 


$$
I(G, v):=\int_{\partial D}\left(v(y) \frac{\partial u}{\partial \nu}(y)-u(y) \frac{\partial v}{\partial \nu}(y)\right) d s(y)
$$

that measures the response according to a scattering process with incident field $v$ in terms of the total field, see [58]. The test domain $G$ is called positive if its response $|I(G, v)|$ is smaller than some constant $c>0$ for all functions $v$ which satisfy the impulse size condition (6.46), otherwise the test domain is called negative. Then, a sampling procedure over all test domains $G$ and all admissible test functions $v$ on $G$ separates the positive from the negative domains and one obtains a reconstruction of the obstacle $D$ from the intersection of all positive test domains.

In the following we will show that the difference $v_{z}=v_{z, \varepsilon_{1}}-v_{z, \varepsilon_{2}}$ of two approximating Herglotz wave functions with different error levels $\varepsilon_{1}, \varepsilon_{2}>0$ satisfies the impulse size condition on the approximation domain $G(z)$ with threshold value $\varepsilon=\varepsilon_{1}+\varepsilon_{2}$. Furthermore we will see that the response $I\left(G, v_{z}\right)$ of this impulse size function is given by

$$
\mathcal{I}_{\mathrm{enl}}(z):=u_{\mathrm{rec}, \varepsilon_{1}}(z)-u_{\mathrm{rec}, \varepsilon_{2}}(z), \quad z \in \mathcal{B} .
$$

The indicator function $\mathcal{I}_{\text {enl }}$ can be seen as a version of the no response test, where we solely use one impulse size function $v$ in the sampling procedure. Obviously, for small error levels both reconstructions $u_{\mathrm{rec}, \varepsilon_{1}}$ and $u_{\mathrm{rec}, \varepsilon_{2}}$ are close to the true solution whenever $\bar{D} \subset G(z)$ holds. In this case the indicator function yields small values and therefore marks the corresponding approximation domain positive (admissible). On the other hand if $\bar{D} \not \subset G(z)$ then the approximation domain is not admissible and the reconstruction formula is affected by an arbitrarily large approximation error due to the highly oscillating behaviour of the approximating Herglotz wave functions outside their domain $G$ of approximation. Now, the indicator function $\mathcal{I}_{\text {enl }}$ will be larger than the threshold value $c$ and we assign the domain $G$ negative. In this manner, the indicator function separates the admissible approximation domains from the nonadmissible ones. Taking the response of all possible impulse size functions into account and intersecting all positive approximation domains would yield the reconstruction algorithm of the no response test. From the estimate

$$
\begin{aligned}
\left\|v_{z, \varepsilon_{1}}-v_{z, \varepsilon_{2}}\right\|_{C^{1}(\bar{G})} & \leq\left\|v_{z, \varepsilon_{1}}-\Phi(\cdot, z)\right\|_{C^{1}(\bar{G})}+\left\|\Phi(\cdot, z)-v_{z, \varepsilon_{2}}\right\|_{C^{1}(\bar{G})} \\
& \leq \varepsilon_{1}+\varepsilon_{2}=\varepsilon
\end{aligned}
$$

we deduce that the Herglotz wave function $v_{z}$ with the density

$$
g_{z}:=g_{z, \varepsilon_{1}}-g_{z, \varepsilon_{2}}
$$

satisfies the impulse size condition (6.46). Hence $v_{z}$ is an admissible test function for the no response test and its response is given by 


$$
\begin{aligned}
I\left(G, v_{z}\right) & =\int_{\partial D}\left(\frac{\partial u}{\partial \nu}(y) v_{z}(y)-\frac{\partial v_{z}}{\partial \nu}(y) u(y)\right) d s(y) \\
& =-\int_{\partial \Omega}\left(\frac{\partial u}{\partial \nu}(y) v_{z}(y)-\frac{\partial v_{z}}{\partial \nu}(y) u(y)\right) d s(y) \\
& =u_{\mathrm{rec}, \varepsilon_{1}}(z)-u_{\mathrm{rec}, \varepsilon_{2}}(z) .
\end{aligned}
$$

Therefore the indicator function $\mathcal{I}_{\text {enl }}(z)$ coincides with the indicator function $I\left(G, v_{z}\right)$ of the no response test. Furthermore, by (6.42) it admits the representation

$$
\mathcal{I}_{\mathrm{enl}}(z)=\int_{\mathbb{S}^{m-1}} J(d) g_{z}(d) d s(d)
$$

with the function

$$
J(d):=-\int_{\partial \Omega}\left(\frac{\partial u}{\partial \nu}(y) e^{i \kappa y \cdot d}-i \kappa d \cdot \nu(y) e^{i \kappa y \cdot d} u(y)\right) d s(y) .
$$

For the static case we obtain a similar formula by substituting the kernel of the static Herglotz wave function and its normal derivative in the previous equation.

In our situation we do not evaluate the indicator functional of the no response test for all admissible test functions but only for one. Hence we can not expect a full reconstruction but nevertheless this procedure provides an indication whether the approximation domain contains the unknown obstacle and is therefore admissible or not. If the response $\left|\mathcal{I}_{\text {enl }}(z)\right|$ is larger than some constant $c>0$ we conclude that the scatterer $D$ is not contained in $\bar{G}$ and therefore the approximation domain is not admissible. In the other case we can not be sure that there might exist another admissible test function for which the response will exceed the threshold value $c$ but we also can not reject the hypothesis that the approximation domain is admissible. For the computation of the indicator function $\mathcal{I}_{\text {enl }}$ we can use the reconstructed value $u_{\mathrm{rec}, \varepsilon}$ and have to perform the point source algorithm another time with a different regularization parameter. Therefore we get the additional information about the admissibility region as a byproduct of the point source method by investing the double amount of work.

Notice that in the original work [58] Potthast used a single-layer potential on the measurement boundary $\partial \Omega$ to construct admissible test functions. However, the no response test is independent of the special type of test functions as long as they both solve the underlying PDE and are dense in the space $L^{2}(\partial G)$, i.e. they have to fulfill the same conditions as mentioned above for the approximation of the point source.

With the indicator function for the admissibility region we will now summarize the point source method for boundary value problems in the following algorithm. We restrict ourselves here to the strategy of choosing the approximation domains as orthogonal translations (5.86) of a fixed reference configuration $\left(0, G_{0}\right)$, where we use (static) Herglotz wave functions for the approximation of the point source. 
Definition 6.8 (Point source method for BVPs). For the boundary value problems (6.1) - (6.3) and (6.4) - (6.6) the point source method calculates an approximation $u_{\mathrm{rec}, \varepsilon}$ to the true field $u$ on subsets $\mathcal{E}_{c}$ of $\mathcal{B}$ by the following steps:

1. Choose a fixed reference configuration $\left(0, G_{0}\right)$ such that $0 \notin G_{0}$ and calculate the density $g_{0, \varepsilon} \in L^{2}\left(\mathbb{S}^{m-1}\right)$ as a solution of equation (5.89) with $H$ defined by (5.45) for the Helmholtz equation and via (5.97) and (5.47) for the Laplace equation.

2. For each $z \in \mathcal{S} \subset \mathcal{B}$ choose a domain of approximation $G(z)$ of the form (5.86), i.e. the configuration $(z, G(z))$ is an orthogonal translation of the reference configuration $\left(0, G_{0}\right)$.

3. For each $z \in \mathcal{S}$ calculate the density $g_{z, \varepsilon} \in L^{2}\left(\mathbb{S}^{m-1}\right)$ via (5.91) for the Helmholtz and via (5.99) for the Laplace equation.

4. For each $z \in \mathcal{S}$ evaluate the Herglotz wave function (5.44) and the static Herglotz wave function (5.48) with density $g_{z, \varepsilon}$ on $\partial \Omega$, respectively.

5. Calculate $u_{\mathrm{rec}, \varepsilon}$ for all $z \in \mathcal{S}$ via (6.23) and (6.24).

6. Repeat steps $3-5$ with a different error level $\varepsilon$ and calculate an approximation $\mathcal{E}_{c}:=\left\{z \in \mathcal{S}:\left|\mathcal{I}_{\text {enl }}(z)\right|<c\right\}$ for the admissibility region.

7. Repeat steps 1 - 6 with a different choice of the approximation domains until the union of the domains $\mathcal{E}_{c}$ is a doubly connected domain. Extend the single reconstructions $u_{\mathrm{rec}, \varepsilon}$ with a suitable weighted mean operation to this domain.

Note that the presented indicator for the admissibility region is an a posteriori scheme. We can not decide whether the reconstructed value is obtained from an admissible approximation domain unless we have performed at least two different reconstructions for the field value in the sampling point. Following this idea one is free to use any other analytic reconstruction method such as the potential method of Kirsch and Kress for crosschecking the quality of this indicator in real applications, where the reconstructed field is not known. Apparently the usage of another analytic reconstruction method for the evaluation of the indicator function is only of theoretical interest since we would have to implement an additional reconstruction algorithm which would increase the computational cost.

For a formulation of the point source method for boundary value problems, where the approximation of the point source is based on a single-layer potential on $\partial \Omega$ we refer to [14]. In this case the computational cost increases since we have to solve the regularized equation (5.35) for each $z \in \mathcal{S}$. Moreover, with an approximation of the point source with a single-layer potential

$$
v_{z, \varepsilon}(x)=\int_{\partial \Omega} \Phi(x, \xi) g_{z, \varepsilon}(\xi) d s(\xi), \quad x \in \Omega
$$

we have to deal with the direct values

$$
v_{z, \varepsilon}(y)=\int_{\partial \Omega} \Phi(y, \xi) g_{z, \varepsilon}(\xi) d s(\xi), \quad y \in \partial \Omega
$$


of the single-layer potential and the direct values of the adjoint double-layer potential in the normal derivative

$$
\frac{\partial v_{z, \varepsilon}}{\partial \nu}(y)=\int_{\partial \Omega} \frac{\partial \Phi(y, \xi)}{\partial \nu(y)} g_{z, \varepsilon}(\xi) d s(\xi)+\frac{1}{2} g_{z, \varepsilon}(y), \quad y \in \partial \Omega
$$

of $v_{z, \varepsilon}$ on the measurement boundary $\partial \Omega$. Inserting (6.55) and (6.56) into the reconstruction formula (6.24) yields

$$
\begin{aligned}
u_{\mathrm{rec}, \varepsilon}(z)= & \int_{\partial \Omega}\left\{\frac{\partial u}{\partial \nu}(y) \Phi(z, y)-\frac{\partial \Phi(z, y)}{\partial \nu(y)} u(y)\right\} d s(y) \\
& +\int_{\partial \Omega} g_{z, \varepsilon}(\xi) h(\xi) d s(\xi)
\end{aligned}
$$

with

$$
h(\xi)=\frac{1}{2} u(\xi)-\int_{\partial \Omega}\left\{\frac{\partial u}{\partial \nu}(y) \Phi(\xi, y)-\frac{\partial \Phi(\xi, y)}{\partial \nu(y)} u(y)\right\} d s(y)
$$

for $z \in \mathcal{E}$.

This approach can be modified by defining the approximating functions $v_{z, \varepsilon}$ as single-layer potentials defined on the boundary of a domain $\Omega^{\prime}$ such that $\bar{\Omega} \subset \Omega^{\prime}$. It is easy to show that Theorem 5.7 and Theorem 5.1 which prove the injectivity and denseness of the single-layer operator $S_{z, \Omega}$ remain valid for the single-layer operator $S_{z \Omega^{\prime}}$ defined on the boundary $\partial \Omega^{\prime}$. Proceeding as before we can find an approximation

$$
v_{z, \varepsilon}(x)=\int_{\partial \Omega^{\prime}} \Phi(x, \xi) g_{z, \varepsilon}(\xi) d s(\xi), \quad x \in \Omega^{\prime}
$$

of the point source $\Phi(\cdot, z)$ on $\partial G(z)$ with this approach, where the density $g_{z, \varepsilon}$ solves the corresponding regularized Tikhonov equation

$$
\left(\alpha I+S_{z, \Omega^{\prime}}^{*} S_{z, \Omega^{\prime}}\right) g_{z, \varepsilon}=\left.S_{z, \Omega^{\prime}}^{*} \Phi(\cdot, z)\right|_{\partial G(z)} .
$$

Using this modification we can calculate the values of $v_{z, \varepsilon}$ on $\partial \Omega \subset \Omega^{\prime}$ by simply evaluating the values of the single-layer potential on the curve $\partial \Omega$ directly. The normal derivative of $v_{z, \varepsilon}$ on $\partial \Omega$ is obtained in the same way by first differentiating the single-layer potential under the integral and then evaluating the expression on $\partial \Omega$. This procedure avoids calculating the direct values of a layer potential and leads to the following reconstruction formula

$$
\begin{aligned}
u_{\mathrm{rec}, \varepsilon}(z)= & \int_{\partial \Omega}\left\{\frac{\partial u}{\partial \nu}(y) \Phi(z, y)-\frac{\partial \Phi(z, y)}{\partial \nu(y)} u(y)\right\} d s(y) \\
& -\int_{\partial \Omega} \int_{\partial \Omega^{\prime}} g_{z, \varepsilon}(\xi)\left\{\frac{\partial u}{\partial \nu}(y) \Phi(\xi, y)-\frac{\partial \Phi(\xi, y)}{\partial \nu(y)} u(y)\right\} d s(\xi) d s(y)
\end{aligned}
$$

for $z \in \mathcal{E}$. 


\subsection{The PSM for Acoustic Scattering Problems}

Having started with the acoustic boundary value problem we will now consider the direct and inverse acoustic scattering problem for a sound-soft obstacle. We begin this section with a formulation of the direct problem before we state some uniqueness results for the inverse acoustic scattering problem. The main goal will be the deduction of the point source method for inverse scattering by investigating the PSM for boundary value problems on large balls $\Omega=B_{r}(0)$. We prove that the PSM for inverse scattering is connected to the PSM for boundary value problems as we apply the limit and let the radius $r$ of the ball $\Omega$ tend to infinity. This approach does not use the mixed reciprocity relation and is therefore applicable in every situation, where we know the far field pattern of an arbitrary incident wave. However, if we do not restrict ourselves to incident plane waves we run into trouble concerning the uniqueness of the inverse scattering problem as we demonstrate with a simple example. Even in the case of one incident plane wave the uniqueness of the inverse problem is still not settled. But we will see that if we search for an obstacle in a domain $\Omega=B_{R}$ of interest with radius $R<\pi / \kappa$ then uniqueness holds for the inverse scattering problem with one incident plane wave.

Acoustic scattering problem. We consider acoustic scattering from a bounded impenetrable scatterer $D$. The scatterer $D$ consists of a domain $D \subset \mathbb{R}^{3}$ and some boundary condition for the total field on the boundary $\partial D$. We will restrict ourselves to the case of a Dirichlet boundary condition, e.g. we only consider scattering obstacles of sound-soft type. Furthermore, we assume the boundary $\partial D$ to be of class $C^{2}$ and the open exterior $\mathbb{R}^{m} \backslash \bar{D}$ of the scatterer to be connected. The unit normal vector $\nu$ to the boundary $\partial D$ is directed into the exterior of $D$. In acoustic scattering we are interested in the response of the scatterer due to some incident field $u^{i}$ which is a solution to the Helmholtz equation

$$
\triangle u+\kappa^{2} u=0
$$

on a domain containing $D$ in its interior. Here we consider the wave number $\kappa$ to be real and positive. We summarize the direct acoustic scattering problem for a sound-soft obstacle in the following definition.

Definition 6.9. Given an incident field $u^{i}$ and a scatterer $D$, the direct acoustic obstacle scattering problem is to find a scattered field

$$
u^{s} \in C^{2}\left(\mathbb{R}^{m} \backslash \bar{D}\right) \cap C\left(\mathbb{R}^{m} \backslash D\right),
$$

which solves the Helmholtz equation (6.62) in $\mathbb{R}^{m} \backslash \bar{D}$ and satisfies the Sommerfeld radiation condition

$$
r^{\frac{m-1}{2}}\left(\frac{\partial u^{s}}{\partial r}-i \kappa u^{s}\right) \rightarrow 0, \quad r=|x| \rightarrow \infty
$$


uniformly in all directions $\hat{x}=x /|x|$, such that the total field

$$
u=u^{i}+u^{s}
$$

satisfies the sound-soft boundary condition

$$
u^{i}+u^{s}=0 \text { on } \partial D .
$$

A solution $u$ of the Helmholtz equation in the exterior of some ball B satisfying (6.64) is called radiating.

It is well known, cf. Theorem 3.9 in [7], that the direct acoustic scattering problem is uniquely solvable and that its solution $u^{s}$ can be represented in terms of a combined single- and double-layer potential

$$
u^{s}(x)=\int_{\partial D}\left(\frac{\partial \Phi(x, y)}{\partial \nu(y)}-i \eta \Phi(x, y)\right) \varphi(y) d s(y), \quad x \in \mathbb{R}^{m} \backslash \bar{D}
$$

on the boundary $\partial D$ with a continuous density $\varphi \in C(\partial D)$ and a positive coupling parameter $\eta>0$.

As we have seen in the previous section Green's representation formula (2.40) was one main ingredient for the analytic reconstruction of the scattered field with the point source method in the inverse acoustic boundary value problem. For the inverse scattering problem we need an extension of this representation formula which holds in the exterior $\mathbb{R}^{m} \backslash \bar{D}$ of the scattering obstacle $D$. Consider Green's representation formula in $\Omega \backslash \bar{D}$ with a large ball $\Omega=B_{r}(0)$ and let the radius $r$ of this ball tend to infinity. Obviously, the contribution from the measurement boundary $\partial \Omega$ can only be neglected if we require some decay condition on the field which is to be represented. In particular for a radiating solution of Helmholtz' equation, e.g. the scattered field $u^{s}$, we obtain the representation formula

$$
u^{s}(x)=\int_{\partial D}\left\{u^{s}(y) \frac{\partial \Phi(x, y)}{\partial \nu(y)}-\frac{\partial u^{s}}{\partial \nu}(y) \Phi(x, y)\right\} d s(y), \quad x \in \mathbb{R}^{m} \backslash \bar{D}
$$

in the unbounded exterior of the scatterer, see Theorem 2.4 in [7].

Moreover, we can deduce the asymptotic behavior of the scattered field which is given by

$$
u^{s}(x)=\frac{e^{i \kappa|x|}}{|x|^{\frac{m-1}{2}}}\left\{u^{\infty}(\hat{x})+O\left(\frac{1}{|x|}\right)\right\}, \quad|x| \rightarrow \infty,
$$

where $\hat{x}:=x /|x| \in \mathbb{S}^{m-1}$ from Green's representation theorem, see [7], Theorem 2.5. The function $u^{\infty}$ depending on the direction $\hat{x}$ only is called the far field pattern of the scattered acoustic wave.

Of particular interest are incident fields $u^{i}$ in form of plane waves 


$$
u^{i}(x, d):=e^{i \kappa x \cdot d}, \quad x \in \mathbb{R}^{m},
$$

with incident direction $d \in \mathbb{S}^{m-1}$ as well as incident fields of point sources $\Phi(\cdot, z)$ with source point $z \in \mathbb{R}^{m} \backslash \bar{D}$. We denote with $u^{s}(x, d), x \in \mathbb{R}^{m} \backslash D$ the scattered field due to an excitation with an incident plane wave $u^{i}(x, d)$ with direction $d \in \mathbb{S}^{m-1}$ and we use the notation $u^{\infty}(\hat{x}, d), \hat{x} \in \mathbb{S}^{m-1}$ for the corresponding far field pattern. Analogously we define the scattered field $\Phi^{s}(x, z), x \in \mathbb{R}^{m} \backslash D$ and the far field pattern $\Phi^{\infty}(\hat{x}, z), \hat{x} \in \mathbb{S}^{m-1}$ caused by an incident point source with source point $z \in \mathbb{R}^{m} \backslash \bar{D}$. We are now prepared to state the problem setting in inverse acoustic scattering.

Inverse acoustic scattering problem. In the inverse acoustic scattering problem we want to identify the scatterer $D$ for fixed wave number $\kappa$ from the knowledge of a set of far field patterns $\left\{u_{j}^{\infty}: j \in \mathcal{J}\right\}$ with a nonempty index set $\mathcal{J}$. Here the index set $\mathcal{J}$ is of the form $\mathcal{J}=\mathbb{N}$, $\mathcal{J}=\{1, \ldots, N\}$ with $N \in \mathbb{N}$ or $\mathcal{J}=\{1\}$ and corresponds to the situation, where we have access to the far field patterns of all incident plane waves, a finite number or at least one incident plane wave.

In the following we present uniqueness results for these three situations. However, it is still an open problem whether the inverse problem of reconstructing the scatterer from the far field pattern of one incident plane wave is unique. Therefore we need additional assumptions on the size of the scatterer in the case of finitely many incident waves to prove uniqueness. In general, if we consider the scattering of one arbitrarily incident wave, uniqueness does not hold as we demonstrate with a simple example.

For the inverse boundary value problem the theorem of Holmgren endowed us with a one-to-one correspondence of the Cauchy data on the measurement boundary with the solution of the corresponding boundary value problem in $\Omega \backslash \bar{D}$. The analog theorem in inverse scattering theory is based on Rellich's lemma, see Lemma 2.11 in [7], and can be formulated as follows.

Theorem 6.10. Let $D$ be a scattering obstacle and $u \in C^{2}\left(\mathbb{R}^{m} \backslash \bar{D}\right)$ be a radiating solution to the Helmholtz equation with vanishing far field pattern. Then $u=0$ in $\mathbb{R}^{m} \backslash \bar{D}$. Furthermore if $u \in C^{2}\left(\mathbb{R}^{m}\right)$ is an entire and radiating solution to the Helmholtz equation then $u=0$ in $\mathbb{R}^{m}$.

Proof. For the proof of the first statement we refer the reader to the monograph [7] of Colton and Kress, Theorem 2.13. We will only prove the second part of Rellich's lemma here.

Let $u$ be an entire and radiating solution to the Helmholtz equation in $\mathbb{R}^{m}$. For every $x \in \mathbb{R}^{m}$ we choose a scattering obstacle $D$ such that $x \notin D$. Since both $\Phi(x, \cdot)$ and $u$ solve the Helmholtz equation in $D$ we obtain $u \equiv 0$ from combining Green's representation formula (6.68) and Green's second formula (2.5).

To show non-uniqueness of the inverse scattering problem in the general case we try to extend Example 6.1 to the scattering setting. Assume that 
the Cauchy data

$$
u(x)=\frac{\sin |x|}{|x|}, \quad \frac{\partial u}{\partial \nu}(x)=\frac{\partial}{\partial \nu} \frac{\sin |x|}{|x|}=\frac{\cos |x|}{|x|}-\frac{\sin |x|}{|x|^{2}}
$$

on $\partial \Omega$ is given as the sum of an entire incident field $u^{i}=u$ defined on $\Omega$ and a vanishing scattered field $u^{s}=0$. The wave number in this example is $\kappa=1$. A measurement of the far field pattern $u^{\infty} \equiv 0$ would indicate at first glance that there is no obstacle. However a spherical obstacle of radius $m \pi$ with $m \in \mathbb{N}$ also produces no response for this special type of incident wave and we are not able to detect such obstacles in this setting. In other words, the "incident to far field mapping" is not injective in general.

We can generalize this example of non-uniqueness in the inverse scattering problem to balls of arbitrary radius which give rise to some non-vanishing scattered field.

Example 6.11. Consider the scattering of a superposition of incident plane waves

$$
u^{i}(x)=\frac{1}{4 \pi} \int_{\mathbb{S}^{2}} e^{i x \cdot d} d s(d), \quad x \in \mathbb{R}^{3}
$$

with wave number $\kappa=1$ by a sound-soft ball $B_{R} \subset \mathbb{R}^{3}$ of radius $R>0$ centered at the origin. From the Funck-Hecke formula

$$
\int_{\mathbb{S}^{2}} e^{-i \kappa r \hat{x} \cdot \hat{z}} Y_{n}(\hat{z}) d s(\hat{z})=\frac{4 \pi}{i^{n}} j_{n}(\kappa r) Y_{n}(\hat{x}), \quad \hat{x} \in \mathbb{S}^{2}, \quad r>0,
$$

applied to the spherical harmonic $Y_{0}=\sqrt{\frac{1}{4 \pi}}$ and the spherical Bessel function $j_{0}(t)=\frac{\sin t}{t}$ we derive

$$
u^{i}(x)=\frac{\sin |x|}{|x|}, \quad x \in \mathbb{R}^{3}
$$

and the scattered wave can be calculated to be

$$
u^{s}(x)=-\frac{\sin R}{e^{i R}} \frac{e^{i|x|}}{|x|}, \quad x \in \mathbb{R}^{3} \backslash D
$$

From

$$
e^{i R} \sin |x|-\sin R e^{i|x|}=\cos R \sin |x|-\sin R \cos |x|
$$

and the addition theorem for the sine-function we can compute the total field

$$
u(x)=\sin (|x|-R) \frac{e^{-i R}}{|x|}, \quad x \in \mathbb{R}^{3} \backslash D .
$$

Hence the total field vanishes on every ball of radius $R_{m}=R+m \pi$ centered at the origin. 
This example illustrates that we can not expect uniqueness of the inverse obstacle scattering problem, when we measure the far field pattern of one arbitrarily incident field. Therefore we restrict ourselves to the case of incident plane waves. However, it is still an open problem if the knowledge of the far field pattern of one incident plane wave is sufficient to identify a scatterer uniquely. Obviously, by Example 6.11 a uniqueness prove would have to make use of special properties of plane waves. In particular, the fact that $u^{i}(x, d) \not \equiv 0$ holds on $\partial D$ for any bounded scatterer $D$ ensures that we will not encounter obstacles that produce no scattered field at all.

There are some indications that uniqueness holds in this case, e.g. the following uniqueness result for convex polyhedra which is often referred to an unpublished work [41] of Liu and Nachman.

Theorem 6.12. Let $D_{1}$ and $D_{2}$ be two polyhedral or polygonal sound-soft scatterers. If for one incident plane wave $u^{i}$ the far field patterns coincide then $D_{1}=D_{2}$.

Recently, Alessandrini and Rondi published a proof of this theorem in [1]. Moreover, Stefanov and Uhlman have proven in [71] the following local uniqueness result for sound-soft scatterers.

Theorem 6.13. Let $D_{-}, D_{+}, D_{1}$ and $D_{2}$ be sound-soft scatterers with boundaries of class $C^{2}$ such that $D_{-} \subset D_{j} \subset D_{+}$for $j=1,2$. Assume that for the volume of the difference set $D_{+} \backslash D_{-}$there holds

$$
\left|D_{+} \backslash D_{-}\right|<\frac{\omega_{m}}{\kappa^{m}},
$$

where $\omega_{n}$ denotes the volume of the unit ball in $\mathbb{R}^{m}$. If the far field patterns coincide for one incident plane wave then $D_{1}=D_{2}$.

Another local uniqueness result which does not dependent on the special type of boundary condition was stated by Potthast in [57]. It states local uniqueness for obstacles in a class of scatterers with certain geometric properties by the knowledge of finite far field data both in observation and in incident direction.

We finally present two well-known global uniqueness results for inverse scattering. The first one states that a scattering obstacle is uniquely determined by the measurement of the far field patterns of all possible incident plane waves.

Theorem 6.14. Let $D_{1}$ and $D_{2}$ be two sound-soft scatterers such that the far field patterns coincide for an infinite number of incident plane waves with distinct directions and one fixed wave number. Then $D_{1}=D_{2}$.

One can proof this theorem with the ideas of Schiffer [38] as demonstrated in [7]. Unfortunately these arguments do not carry over to the sound-hard case without an additional assumption on the smoothness of the boundary of the scatterer. While Isakov [27] settled uniqueness for the inverse transmission 
scattering problem Kirsch and Kress simplified Isakov's proof and applied it to the Neumann problem in [30].

For a finite number of incident plane waves we obtain the analog uniqueness statement to Theorem 6.3 and Corollary 6.4 in the scattering problem, which is proven for example in [7].

Theorem 6.15. Assume that the far field patterns of two sound-soft obstacles $D_{1}, D_{2} \subset \mathbb{R}^{3}$ which are contained in ball $B_{R}$ of radius $R>0$ coincide for $N$ incident plane waves with different directions and one fixed wave number, where

$$
N:=\sum_{t_{n l}<\kappa R}(2 n+1)
$$

is defined as in Theorem 6.3. Then $D_{1}=D_{2}$.

Furthermore if $\kappa R<\pi$ holds for the radius of the ball containing the two scatterers and if the far field patterns coincide for one incident plane wave then $D_{1}=D_{2}$.

Similar to the boundary value problem, the work [18] of Gintides improves the number $N$ of incident plane waves in the previous theorem approximately by the factor two. Furthermore, a two-dimensional analogon to this theorem can be derived in straightforward way as indicated in the remark after Corollary 6.4.

Let us now assume that the sound-soft scatterer is contained in some ball of radius $R>0$, i.e. the a priori information $D \subset B_{R}$ is available. Then we are in the situation of the previous theorem and the scatterer is uniquely determined by the far field pattern of one incident plane wave with wave number $\kappa<\pi / R$. In this case we may try to extend formula (6.24) for the analytic continuation of the Cauchy data for the boundary value problem to the inverse scattering setting.

From Green's representation formula (6.68) for the scattered field together with Green's second theorem applied to the point source $\Phi(x, \cdot)$ with source point $x \in \mathbb{R}^{m} \backslash \bar{D}$ and the incident field $u^{i}$ in $D$ we obtain

$$
u^{s}(x)=\int_{\partial D}\left(\frac{\partial \Phi(x, y)}{\partial \nu(y)} u(y)-\Phi(x, y) \frac{\partial u}{\partial \nu}(y)\right) d s(y), \quad x \in \mathbb{R}^{m} \backslash \bar{D} .
$$

Inserting the sound-soft boundary condition $u=0$ on $\partial D$ this equation simplifies to

$$
u^{s}(x)=-\int_{\partial D} \Phi(x, y) \frac{\partial u}{\partial \nu}(y) d s(y), \quad x \in \mathbb{R}^{m} \backslash \bar{D} .
$$

Thus we can deduce the far field representation

$$
u^{\infty}(\hat{x})=-\int_{\partial D} \Phi^{\infty}(\hat{x}, y) \frac{\partial u}{\partial \nu}(y) d s(y), \quad \hat{x} \in \mathbb{S}^{m-1},
$$


where

$$
\Phi^{\infty}(\hat{x}, z)=\gamma_{m} e^{-i \kappa \hat{x} \cdot z}, \quad \hat{x} \in \mathbb{S}^{m-1}
$$

is the far field pattern of a point source $\Phi(\cdot, z)$ with source point $z \in \mathbb{R}^{m}$ and the constants $\gamma_{m}$ are given by

$$
\gamma_{2}=\frac{e^{i \frac{\pi}{4}}}{\sqrt{8 \pi \kappa}}, \quad \gamma_{3}=\frac{1}{4 \pi}
$$

To obtain an analytic continuation formula for the scattered field we first substitute the point source $\Phi(x, \cdot)$ in $(6.81)$ with an approximating Herglotz wave function

$$
v_{x, \varepsilon}(y):=\int_{\mathbb{S}^{m-1}} e^{i \kappa y \cdot d} g_{x, \varepsilon}(d) d s(d), \quad y \in \mathbb{R}^{m},
$$

such that the estimate

$$
\left\|v_{x, \varepsilon}-\Phi(x, \cdot)\right\|_{C^{1}(\partial D)}<\varepsilon
$$

holds true. This yields

$$
\begin{aligned}
-\int_{\partial D} v_{x, \varepsilon}(y) \frac{\partial u}{\partial \nu}(y) d s(y) & =-\int_{\partial D} \int_{\mathbb{S}^{m-1}} e^{i \kappa y \cdot d} g_{x, \varepsilon}(d) d s(d) \frac{\partial u}{\partial \nu}(y) d s(y) \\
& =-\int_{\mathbb{S}^{m-1}} \int_{\partial D} e^{i \kappa y \cdot d} \frac{\partial u}{\partial \nu}(y) d s(y) g_{x, \varepsilon}(d) d s(d) \\
& =\frac{1}{\gamma_{m}} \int_{\mathbb{S}^{m-1}} u^{\infty}(-d) g_{x, \varepsilon}(d) d s(d)
\end{aligned}
$$

where we have interchanged the order of integration and inserted the representation (6.82) for the far field pattern. Hence the right hand side of (6.87) is an approximation of the true scattered field which can be calculated from the knowledge of the far field pattern of the corresponding incident wave. Thus we define the reconstructed scattered field in the inverse acoustic setting by

$$
u_{\mathrm{rec}, \varepsilon}^{s}(x):=\frac{1}{\gamma_{m}} \int_{\mathbb{S}^{m-1}} u^{\infty}(-d) g_{x, \varepsilon}(d) d s(d)
$$

for all points $x$ in the domain $\mathcal{B}$ of interest.

We obtain convergence of the PSM in inverse scattering analogously to its version for the boundary value problem since the proof of Theorem 6.7 carries over straightforwardly to a convergence proof for the PSM in inverse scattering.

Theorem 6.16. Let $\mathcal{E} \subset \mathcal{B}$ be an enlighted area. Then the reconstructed scattered field (6.88) converges uniformly to the true scattered field on $\mathcal{E}$, i.e.

$$
\lim _{\varepsilon \rightarrow 0} \max _{z \in \mathcal{E}}\left|u^{s}(z)-u_{\mathrm{rec}, \varepsilon}^{s}(z)\right|=0
$$


The continuation formula (6.88) provides a reconstruction algorithm for the unknown scattered field in the domain of interest that uses the far field pattern of an arbitrarily incident wave as input data. This reconstruction formula can be seen to be the natural extension of the corresponding formula (6.24) for the analytic continuation of the total field from its Cauchy data on a measurement boundary as we demonstrate in the following.

To this end we consider the inverse boundary value problem for a spherical domain of interest $\mathcal{B}=B_{r}$ of radius $r>0$, centered at the origin. We do not make any assumptions on the type of boundary condition of the scatterer but we assume the Cauchy data to originate from a scattering process, i.e. the measured data is the sum of a known incident and an unknown scattered wave. Recall that the unit normal vector $\nu$ is directed into the exterior of the scatterer $D$ and into the exterior of the ball $B_{r}$ respectively.

With the analytic continuation formula (6.24) applied to the Cauchy data of the scattered field using $w_{\varepsilon}=\Phi-v_{z, \varepsilon}$ we obtain the reconstruction formula

$$
\begin{aligned}
u_{\mathrm{rec}, \varepsilon}^{s}(z)= & \int_{\partial B_{r}}\left(\frac{\partial u^{s}}{\partial \nu}(y) \Phi(z, y)-\frac{\partial \Phi(z, y)}{\partial \nu(y)} u^{s}(y)\right) d s(y) \\
& -\int_{\partial B_{r}}\left(\frac{\partial u^{s}}{\partial \nu}(y) v_{z, \varepsilon}(y)-\frac{\partial v_{z, \varepsilon}}{\partial \nu}(y) u^{s}(y)\right) d s(y)
\end{aligned}
$$

for the scattered field in $B_{r} \backslash \bar{D}$. Since both functions $v_{z, \varepsilon}$ and $u^{s}$ solve the Helmholtz equation in $B_{r} \backslash \bar{D}$ for fixed $z \in B_{r} \backslash \bar{D}$ we can apply Green's second formula to shift the second integral from the integration domain $\partial B_{r}$ to an integration over the boundary $\partial D$ of the scatterer, which results in

$$
\begin{aligned}
u_{\mathrm{rec}, \varepsilon}^{s}(z) & =\int_{\partial B_{r}}\left(\frac{\partial u^{s}}{\partial \nu}(y) \Phi(z, y)-\frac{\partial \Phi(z, y)}{\partial \nu(y)} u^{s}(y)\right) d s(y) \\
& -\int_{\partial D}\left(\frac{\partial u^{s}}{\partial \nu}(y) v_{z, \varepsilon}(y)-\frac{\partial v_{z, \varepsilon}}{\partial \nu}(y) u^{s}(y)\right) d s(y) .
\end{aligned}
$$

Now we show the first integral to vanish in the limit $r \rightarrow \infty$. To this end we rewrite the first integral in a way that we can apply the radiation condition as follows

$$
\begin{gathered}
\int_{\partial B_{r}}\left(\frac{\partial u^{s}}{\partial \nu}(y) \Phi(z, y)-\frac{\partial \Phi(z, y)}{\partial \nu(y)} u^{s}(y)\right) d s(y) \\
=-\int_{\partial B_{r}}\left(\frac{\partial \Phi(z, y)}{\partial \nu(y)}-i \kappa \Phi(z, y)\right) u^{s}(y) d s(y) \\
\quad+\int_{\partial B_{r}} \Phi(z, y)\left(\frac{\partial u^{s}}{\partial \nu}(y)-i \kappa u^{s}(y)\right) d s(y)
\end{gathered}
$$

Hence, the radiation condition (6.64) together with the asymptotical behaviour of $\Phi$ implies that the integral over the boundary $\partial B_{r}$ in (6.91) vanishes if we let $r$ tend to infinity. Therefore we obtain the representation 


$$
u_{\mathrm{rec}, \varepsilon}^{s}(z)=-\int_{\partial D}\left(\frac{\partial u^{s}}{\partial \nu}(y) v_{z, \varepsilon}(y)-\frac{\partial v_{z, \varepsilon}}{\partial \nu}(y) u^{s}(y)\right) d s(y)
$$

for the reconstructed scattered field in $\mathbb{R}^{m} \backslash \bar{D}$. Inserting the definition of the Herglotz wave function $v_{z, \varepsilon}$ and interchanging the order of integration yields the expression

$$
-\int_{\mathbb{S}^{m-1}} \int_{\partial D}\left(\frac{\partial u^{s}}{\partial \nu}(y) e^{i \kappa y \cdot d}-i \kappa d \cdot \nu(y) e^{i \kappa y \cdot d} u^{s}(y)\right) d s(y) g_{z, \varepsilon}(d) d s(d)
$$

for $u_{\mathrm{rec}, \varepsilon}^{s}(z)$. A comparison with the analog far field representation to (6.82) for an arbitrary boundary condition shows that the inner integral of (6.95) equals the far field pattern. More precisely, there holds

$$
\int_{\partial D}\left(\frac{\partial u^{s}}{\partial \nu}(y) e^{i \kappa y \cdot d}-i \kappa d \cdot \nu(y) e^{i \kappa y \cdot d} u^{s}(y)\right) d s(y)=-\frac{1}{\gamma_{m}} u^{\infty}(-d) .
$$

Therefore we finally derive the analytic continuation formula

$$
u_{\mathrm{rec}, \varepsilon}^{s}(z)=\frac{1}{\gamma_{m}} \int_{\mathbb{S}^{m-1}} u^{\infty}(-d) g_{z, \varepsilon}(d) d s(d)
$$

for the reconstruction of the scattered field in $\mathbb{R}^{m} \backslash \bar{D}$ once again. The reconstruction formula is also often referred to as a backprojection formula since the the right hand side defines an operator that maps the far field pattern "back" to the desired scattered field. We emphasize the independence of this derivation of the PSM from both the boundary condition of the scatterer and the type of incident field. In particular we avoided to use the mixed reciprocity relation

$$
\Phi^{\infty}(-d, z)=\gamma_{m} u^{s}(z, d), \quad z \in \mathbb{R}^{m} \backslash \bar{D}, d \in \mathbb{S}^{m-1},
$$

with $\gamma_{m}$ defined in (6.84), which is tailor-made for scattering of incident plane waves. The main idea to base this proof on Green's theorem, which enables the point source method to reconstruct arbitrary scattered fields, is originally due to Liu [42].

Finally, we summarize the point source method for inverse scattering using orthogonal translations of a fixed reference configuration $\left(0, G_{0}\right)$ in the following algorithm.

Definition 6.17 (Point source method for inverse scattering). For the inverse acoustic scattering problem the point source method calculates an approximation $u_{\mathrm{rec}, \varepsilon}^{s}$ to the true scattered field $u^{s}$ on subsets $\mathcal{E}_{c}$ of $\mathcal{B}$ by the following steps:

1. Choose a fixed reference configuration $\left(0, G_{0}\right)$ such that $0 \notin G_{0}$ and calculate the density $g_{0, \varepsilon} \in L^{2}\left(\mathbb{S}^{m-1}\right)$ as a solution of equation (5.89) with $H$ defined by (5.45). 
2. For each $z \in \mathcal{S} \subset \mathcal{B}$ choose a domain of approximation $G(z)$ of the form (5.86), i.e. the configuration $(z, G(z))$ is an orthogonal translation of the reference configuration $\left(0, G_{0}\right)$.

3. For each $z \in \mathcal{S}$ calculate the density $g_{z, \varepsilon} \in L^{2}\left(\mathbb{S}^{m-1}\right)$ via (5.91).

4. Calculate $u_{\mathrm{rec}, \varepsilon}^{s}$ for all $z \in \mathcal{S}$ via (6.88).

5. Repeat steps $2-4$ with a different error level $\varepsilon$ and calculate an approximation $\mathcal{E}_{c}:=\left\{z \in \mathcal{S}:\left|\mathcal{I}_{\text {enl }}(z)\right|<c\right\}$ for the admissibility region with the indicator function $\mathcal{I}_{\text {enl }}$ given by (6.48).

6. Repeat steps 1 - 5 with a different choice of the approximation domains until the union of the domains $\mathcal{E}_{c}$ is a doubly connected domain. Extend the single reconstructions $u_{\mathrm{rec}, \varepsilon}^{s}$ with a suitable weighted mean operation to this domain.

\subsection{Numerical Examples}

We will illustrate the point source method for obstacle reconstructions with some numerical examples in the boundary value and in the inverse scattering problem setting. First, we demonstrate Algorithm 6.8 in the twodimensional configuration of Section 4.2, see Figure 4.1, for the Laplace equation using the static Herglotz wave approximation technique. The obstacle reconstruction problem for the Helmholtz boundary value problem is then considered with a wavelength comparable to the scatterer's size. We present numerical examples in this case and, finally, illustrate the PSM in inverse acoustic 3D scattering.

Let us start with the inverse boundary value problem for the Laplace equation in two dimensions. If we do not have any information about the shape or size of the obstacle, it is advisable to choose a large and uniform approximation domain in the first step of Algorithm 6.8. In the setting of Figure 4.1, the measurement boundary is a circle of radius $r=1$. Hence the choice of a circular approximation domain $G_{0}$ of the same radius ensures that we definitely obtain a non-empty admissibility region. To satisfy the condition $0 \notin G_{0}$ we center the circle in the point $\left(r+\varepsilon_{r}, 0\right)$ such that it is in distance $\varepsilon_{r}=0.1$ of the source point $z=0$.

Then we calculate an approximation of $\Phi(\cdot, 0)$ on $G_{0}$ by solving the regularized integral equation

$$
\left(\alpha I+H^{*} H\right) g_{0, \varepsilon}=H^{*} \Phi(\cdot, 0)
$$

on $L^{2}\left(\partial G_{0}\right)$ for $g_{0, \varepsilon}$ with Nyström's method. Here, the static Herglotz wave operator $H$ and its adjoint operator $H^{*}$ are given by (5.47) and (5.73), respectively, where we use the trapezoidal rule for their numerical implementation. Figure 6.1 shows the fundamental solution $\Phi(\cdot, 0)$ and its approximation 


$$
v(x)=\operatorname{Re} \int_{\mathbb{S}^{1}} e^{i\left(i d+d^{\perp}\right) \cdot x} g_{0, \varepsilon}(d) d s(d), \quad x \in \mathbb{R}^{2}
$$

on $[-0.5,2.5] \times[-1.5,1.5]$, where we have used the regularization parameter $\alpha=10^{-8}$ and $n=50$ points on both boundaries $\mathbb{S}^{1}$ and $\partial G_{0}$. Moreover,
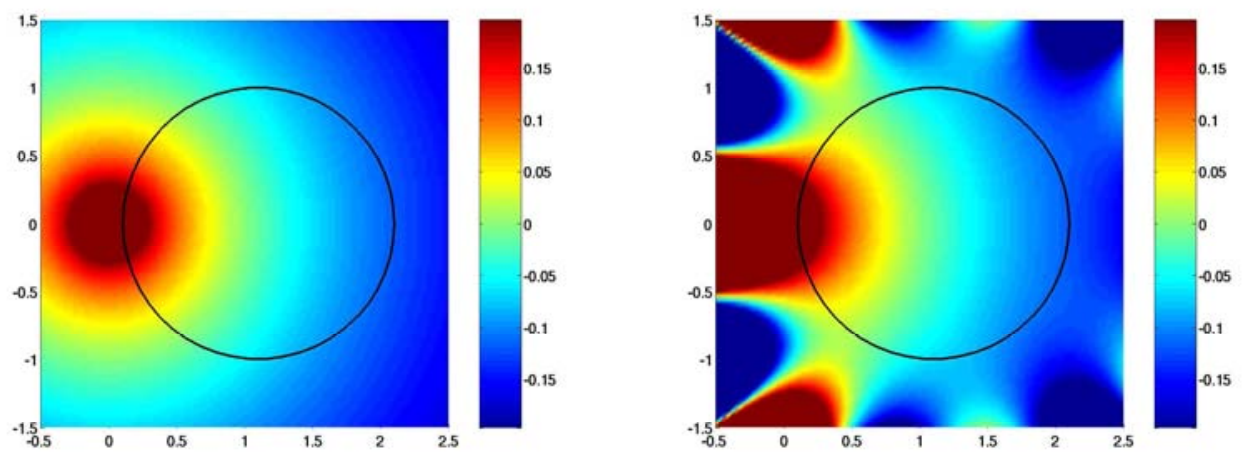

Fig. 6.1. Fundamental solution $\Phi(\cdot, 0)$ (left) and its approximation $v$ (right) for the circular approximation domain $G_{0}$.

Figure 6.2 illustrates the strongly oscillating behaviour of the approximation error outside the domain $G_{0}$. Moreover, we observe that the quality of approximation is best in the center of the approximation domain.
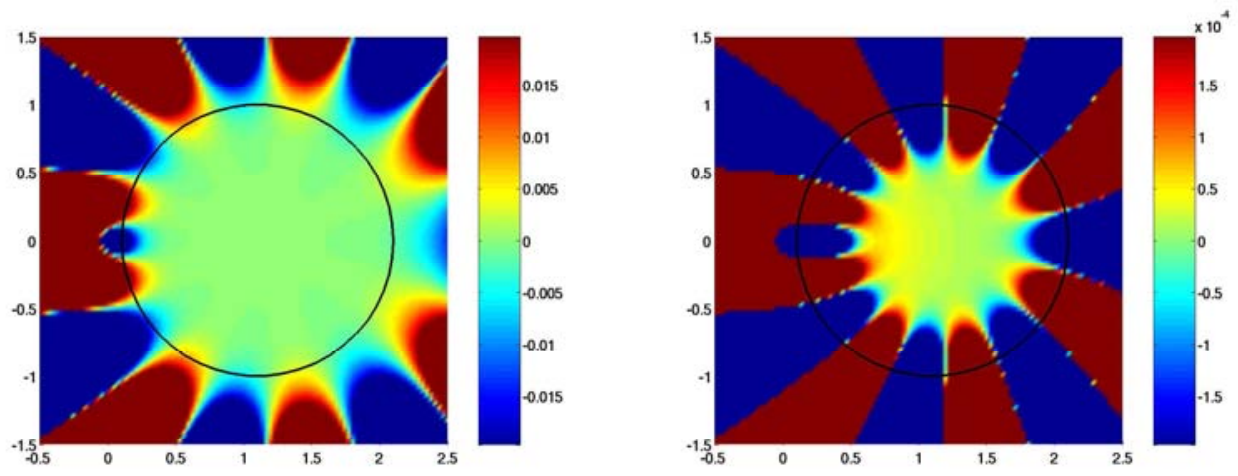

Fig. 6.2. Difference $v-\Phi(\cdot, 0)$ between the approximation $v$ and the point source $\Phi(\cdot, 0)$ for two different settings of the colorbar.

According to the second step in Algorithm 6.8 we now choose the approximation domain $G(z)=z+G_{0}$ for every $z$ in some set $\mathcal{S} \subset \Omega$ of evaluation points. To this end we use the strategy of translations (5.115) for the choice of the approximation domains, i.e. the configuration $(z, G(z))$ arises from the configuration $\left(0, G_{0}\right)$ by a translation with the vector $z$. In this case we can calculate the densities $g_{z, \varepsilon}$ via the multiplication

$$
g_{z, \varepsilon}(d)=e^{-i\left(i d+d^{\perp}\right) \cdot z} g_{0, \varepsilon}(d), \quad d \in \mathbb{S}^{1} .
$$


As Figures 6.1 and 6.2 indicate, the approximation of the point source is valid on $G(z)$ - the circle of radius $r=1$ in distance $\varepsilon_{r}=0.1$ of the source point $z$. Hence, we can expect a valid reconstruction of the field in the admissibility region which will be located on the left side of the obstacle $D$ for this configuration. On the other hand, if we consider a source point $z$ outside the admissibility region, the oscillating behavior of the difference function $w$ will affect the reconstruction formula (6.24). More precisely, we can not neglect the contribution from the obstacle's boundary $\partial D$ in the reconstruction formula and we are faced with the error

$$
\left|u_{\mathrm{rec}, \varepsilon}(z)-u(z)\right|=\left|\int_{\partial D} w(x, z) \frac{\partial u}{\partial \nu}(x) d s(x)\right| .
$$

Due to the behaviour of the function $w$ outside the approximation domain $G$ we expect an increasing error when the source point moves out of the admissibility region. A detailed study of this expression is presented in the convergence proof of the no response test by Potthast, see [58].

For the reconstruction of the field $u$ we use the trapezoidal rule for the evaluation of the reconstruction formula (6.24). As input data, we calculate the direct problem (4.83) - (4.85) with the boundary values $f=4+x_{1}$ for points $\left(x_{1}, x_{2}\right)$ on the measurement boundary $\partial \Omega$ in the three settings of Figure 4.1. To generate synthetic input data we need to compute the normal derivative of the electric field on the measurement boundary by differentiating the ansatz function, which we describe in the next chapter. We mention that one can avoid to differentiate the double-layer potential, which leads to a hypersingular integral operator, by first solving the boundary value problem on an auxiliary boundary curve $\partial \Omega^{\prime}$ with $\Omega \subset \Omega^{\prime}$, and then computing the normal derivative on $\partial \Omega$ numerically.

Figure 6.3 shows the numerical results for Setting 1 (ellipse) on a grid of 100 by 100 points. In particular the reconstruction of the obstacle's boundary as the zero levelset of the electric field is indicated in the second row by a suitable choice of the color scale. The left part of the ellipse is reconstructed quite well as we did expect. To estimate the region where the approximation of the point source is valid we start a second run of the PSM algorithm with the regularization parameter $\alpha=2 \cdot 10^{-8}$. With the indicator function (6.48) of the admissibility region we obtain a measure for the quality of the point source approximation in $\Omega$, which is illustrated together with the reconstruction error $u_{\mathrm{rec}, \varepsilon}-u$ in Figure 6.4. Hence, we will judge the quality of the reconstruction in the following examples by the values of the indicator function $\mathcal{I}_{\text {enl }}$.

In the post-processing step, we first restrict the reconstructed field to the enlighted area

$$
\mathcal{E}_{c}:=\left\{z \in \mathcal{S}:\left|\mathcal{I}_{\text {enl }}(z)\right|<c\right\}
$$



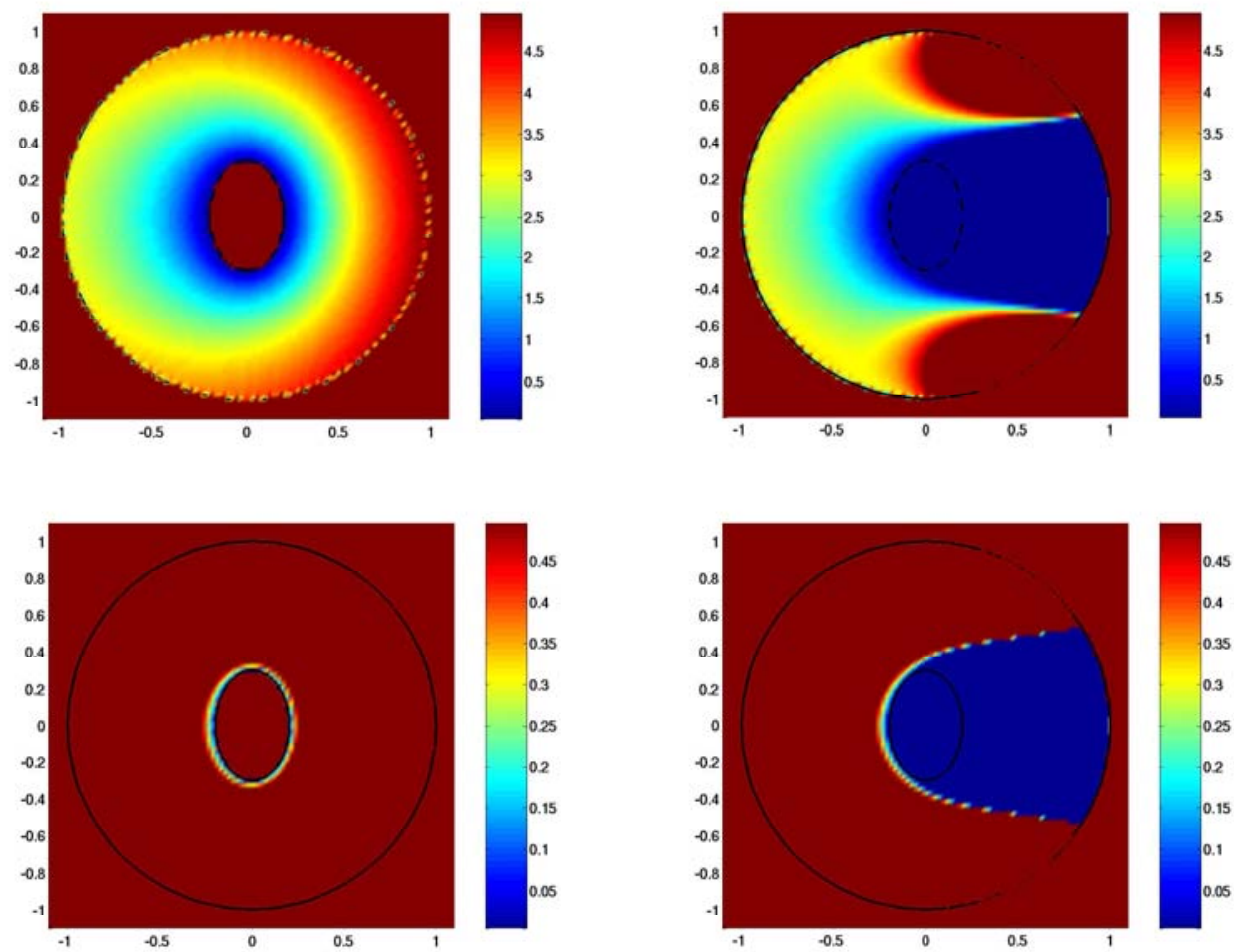

Fig. 6.3. Solution $u$ (left) of the direct problem and its reconstruction $u_{\text {rec, } \varepsilon}$ (right) for Setting 1 (ellipse).
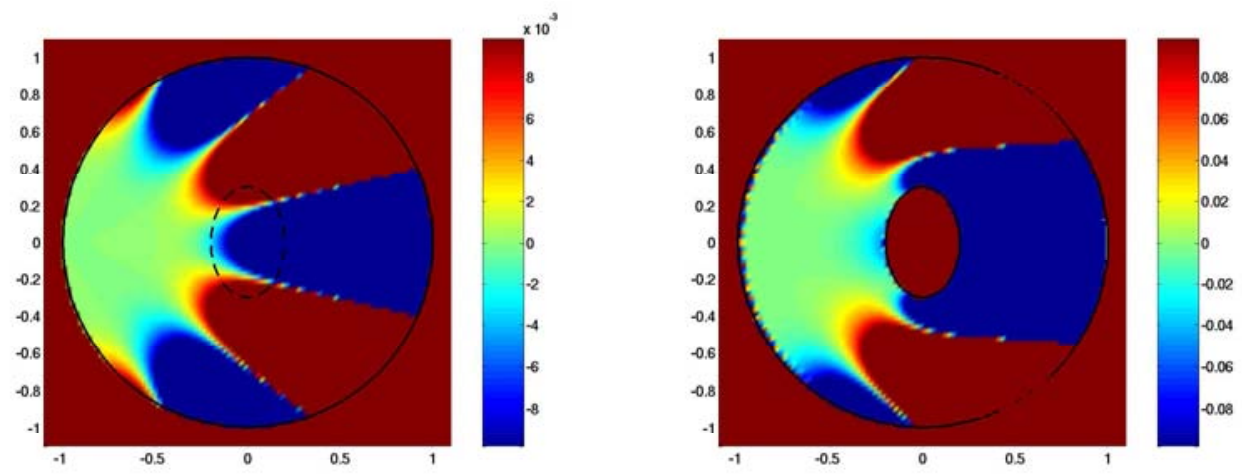

Fig. 6.4. The left figure shows the difference $u_{\mathrm{rec}, \varepsilon_{1}}-u_{\mathrm{rec}, \varepsilon_{2}}$ of two reconstructions with different regularization parameter while the right figure illustrates the error $u_{\mathrm{rec}, \varepsilon_{1}}-u$ between the reconstructed and the true field. 
where we choose the cut-off parameter $c=0.01$ as suggested by Figure 6.4. This region consists of a convex circular main part with additional rays into its complement, see Figure 6.5. While the convex part indicates the region where the indicator function is slowly varying and the admissibility condition $D \subset G(z)$ is satisfied, the additional rays arise from the oscillatory behaviour of the indicator function outside this region and should therefore be ignored in the reconstruction process. Hence, we modify the enlighted area by fitting a disk $B_{r}\left(z_{0}\right)$ into $\mathcal{E}_{c}$ with radius

$$
r=q \sqrt{\frac{\int_{\mathcal{E}_{c}} 1 d x}{\pi}}
$$

and center given as the barycenter

$$
z_{0}=\frac{\int_{\mathcal{E}_{c}} x d x}{\int_{\mathcal{E}_{c}} 1 d x}
$$

of $\mathcal{E}_{c}$. Here $q<1$ is s positive constant that controls the radius of the disk. Figure 6.5 illustrates both the enlighted area $\mathcal{E}_{c}$ and the disk $B_{r}\left(z_{0}\right)$ with
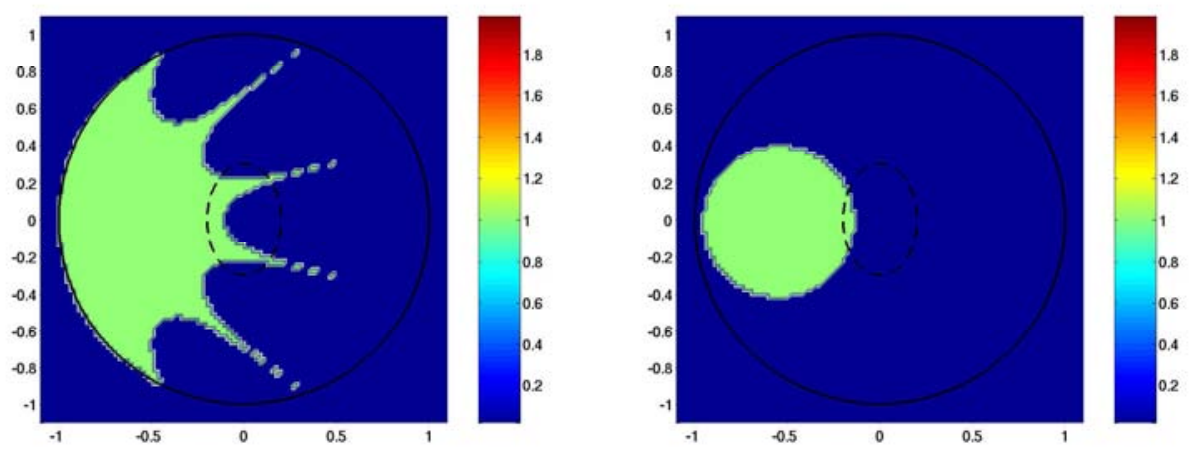

Fig. 6.5. The left figure shows the enlighted area $\mathcal{E}_{c}$ with the cut parameter $c=0.01$ while the right figure shows the disk $B_{r}\left(z_{0}\right)$ with the control parameter $q=0.7$.

$q=0.7$ and $c=0.01$ while Figure 6.6 compares the reconstruction $u_{\mathrm{rec}, \varepsilon}$ and the true field $u$ in the disk $B_{r}\left(z_{0}\right)$. We mention here that the disk is not centered in the region $\left\{z \in \mathbb{R}^{2}:\left|\mathcal{I}_{\text {enl }}(z)\right|<c\right\}$ since the enlighted are $\mathcal{E}_{c}$ is only defined for $z \in \mathcal{S} \subset \Omega$. This also explains the non-radial behaviour of the error in Figure 6.6. However, the numerical evaluation of the enlighted area outside the domain of interest would lead to an additional computational cost without any principle numerical enhancement of the method. Finally, we obtain a complete reconstruction by repeating this procedure with different reference configurations of the approximation domain in the following way. We choose 8 equi-distributed directions $\theta_{j}=\frac{j \pi}{4}$ for $j=0, \ldots, 7$ and rotate the starting reference configuration $\left(0, G_{0}\right)$ around 

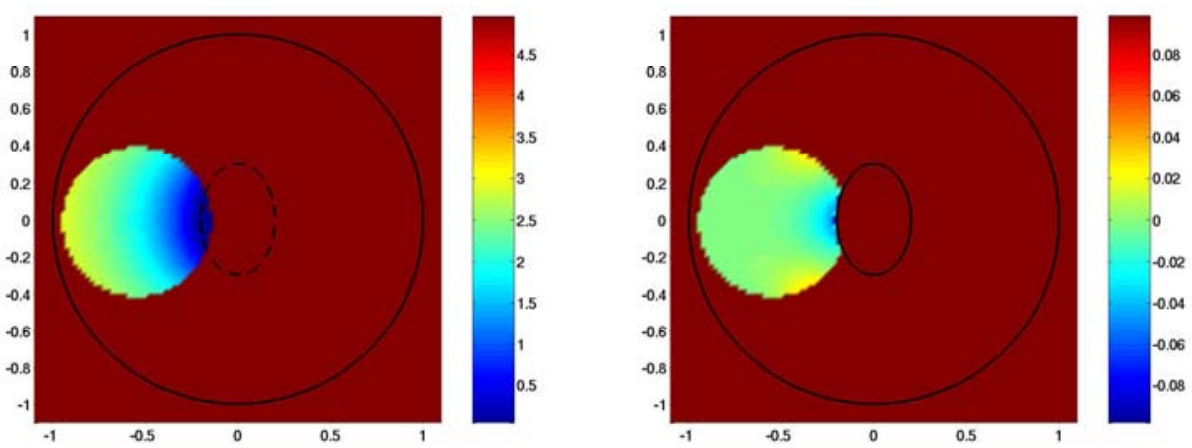

Fig. 6.6. The restriction of the reconstructed field $u_{\mathrm{rec}, \varepsilon}$ to the disk $B_{r}\left(z_{0}\right)$ is plotted in the left figure. The right figure shows the error $u_{\mathrm{rec}, \varepsilon_{1}}-u$ between the reconstructed and the true solution, both restricted to the disk $B_{r}\left(z_{0}\right)$.

the origin by the angle $\theta_{j}$ which gives rise to the rotated configuration domains $\left(0, G_{0}^{(j)}\right)$. For each configuration $\left(0, G_{0}^{(j)}\right)$ we calculate the reconstructed field $u_{\mathrm{rec}, \varepsilon}^{(j)}$ in the corresponding enlighted area $\mathcal{E}_{c}^{(j)}$ with the strategy of translations (5.115) as explained before. Here and in the following, expressions of the form $A^{(j)}$ are understood in the same way as their counterparts $A$, where the reference domain $\left(0, G_{0}\right)$ has to be replaced by $\left(0, G_{0}^{(j)}\right)$. Using this notation for $A=\omega_{\tau}$ we first calculate the functions

$$
\omega_{\tau}^{(j)}(z):=\left\{\begin{array}{cl}
e^{-\tau\left\|z-z_{0}^{(j)}\right\|^{2}}, & z \in \mathcal{S} \cap B^{(j)} \\
0 & , \quad z \in \mathcal{S} \backslash B^{(j)}
\end{array}\right.
$$

and herefrom the weight functions

$$
\tilde{\omega}_{\tau}^{(j)}(z)=\frac{\omega_{\tau}^{(j)}(z)}{\sum_{j=0}^{7} \omega_{\tau}^{(j)}(z)}, \quad z \in \mathcal{S},
$$

for $j=0, \ldots, 7$ and $\tau>0$. These functions take care of the fact that the field values are reconstructed more precisely near the center $z_{0}^{(j)}$ of the disk $B^{(j)}=B_{r^{(j)}}\left(z_{0}^{(j)}\right)$ and are reconstructed with a higher error level when the source point $z$ moves away from the center towards the boundary of the enlighted area. Then a complete reconstruction

$$
u_{\mathrm{rec}, \varepsilon}(z)=\sum_{j=0}^{7} u_{\mathrm{rec}, \varepsilon}^{(j)}(z) \tilde{\omega}_{\tau}^{(j)}(z), \quad z \in \mathcal{S},
$$

of the field is given as the weighted mean of the reconstructed fields $u_{\mathrm{rec}, \varepsilon}^{(j)}$ with the weight functions $\tilde{\omega}_{\tau}^{(j)}(z)$ for $j=0, \ldots, 7$.

Figure 6.7 shows the complete reconstruction together with the error on the union of the disks $B^{(j)}$ with the weight parameter $\tau=100$. From the reconstructed field we finally obtain an approximation for the boundary of the 

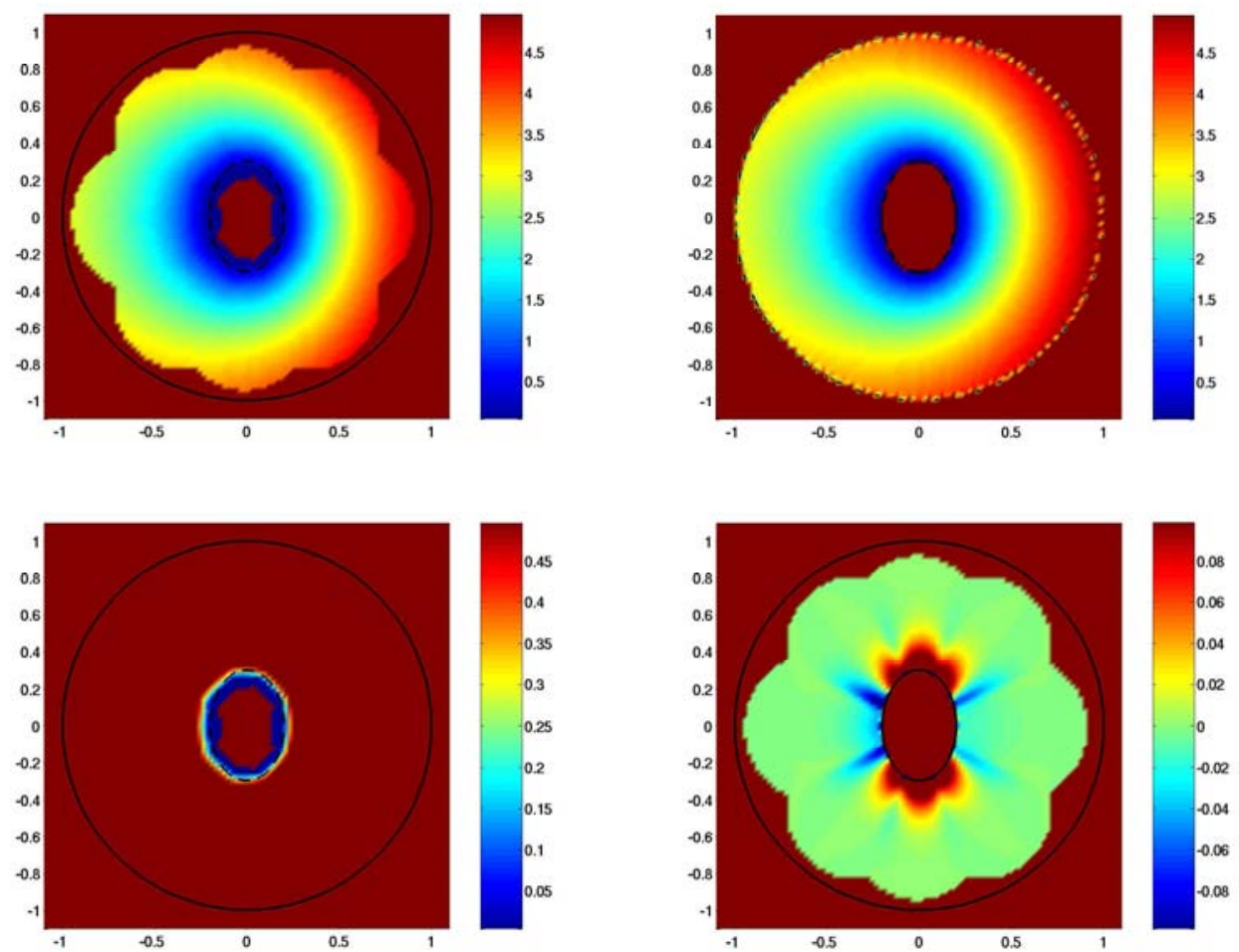

Fig. 6.7. First row: the left figure shows the complete reconstruction $u_{\mathrm{rec}, \varepsilon}$ with the weighted mean (6.108) while the right figure shows the original field. Second row: the left figure illustrates the reconstruction of the obstacle's boundary, the right figure shows the difference of the reconstruction with the true field $u$.
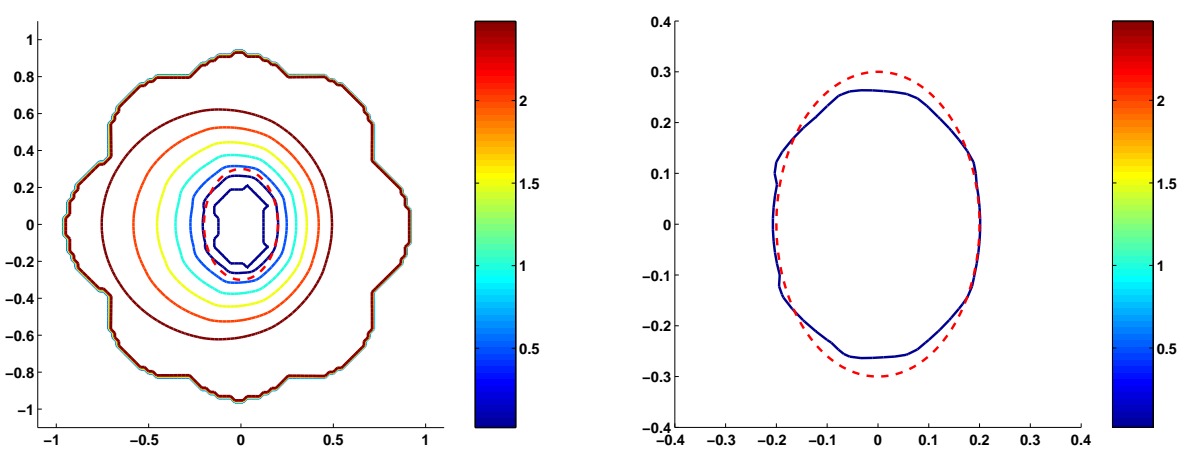

Fig. 6.8. The level sets for the values $0,0.5,1,1.5,2,2.5$ are shown in the left part of the figure. The right part of the figure shows the final reconstruction of the obstacle's boundary which is given by the zero levelset of the field. The boundary $\partial D$ of the obstacle is indicated as a red dashed line. 
perfect conducting obstacle $D$ by searching for zeros of the reconstructed

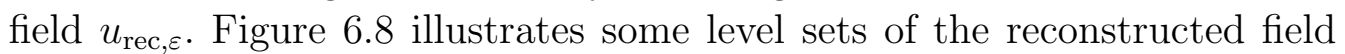
and the final reconstruction of the obstacle's boundary $\partial D$. Outside the union of the disks $B_{r}\left(z_{0}^{(j)}\right)$ we set the values of the reconstructed field to the value 5 . This explains the occurrence of the interior curve inside the obstacle $D$ in the left plot of Figure 6.8.

While the point source method provides highly accurate reconstructions in the admissibility region $\mathcal{A}$, the error increases rapidly when the admissibility condition $\bar{D} \subset G(z)$ is invalidated and makes a precise reconstruction of the obstacle by its boundary values difficult as Figure 6.7 and Figure 6.8 demonstrate. In particular we see that the error does not behave uniformly with respect to the different translation directions which is a consequence of (6.102). Roughly speaking, the error is proportional to a weighted integral of the normal derivative of the electric field over the part $\partial D \backslash G(z)$ of the obstacle's boundary which is not contained in the approximation domain. Since the weight function of this error is given by the quality $w$ of the approximation of the point source on this part of the boundary we expect a better reconstruction when we use approximation domains with a similar ellipsoidal geometry. To this end we use 10 different configurations for the point source approximation as illustrated in Figure 6.9. Repeating the reconstruction algorithm for these ellipsoidal approximation domains validates this argument and provides an accurate boundary reconstruction, see Figure 6.10. For the reconstruction we used the parameters $\tau=100, c=0.7$ and $\alpha=10^{-6}$.
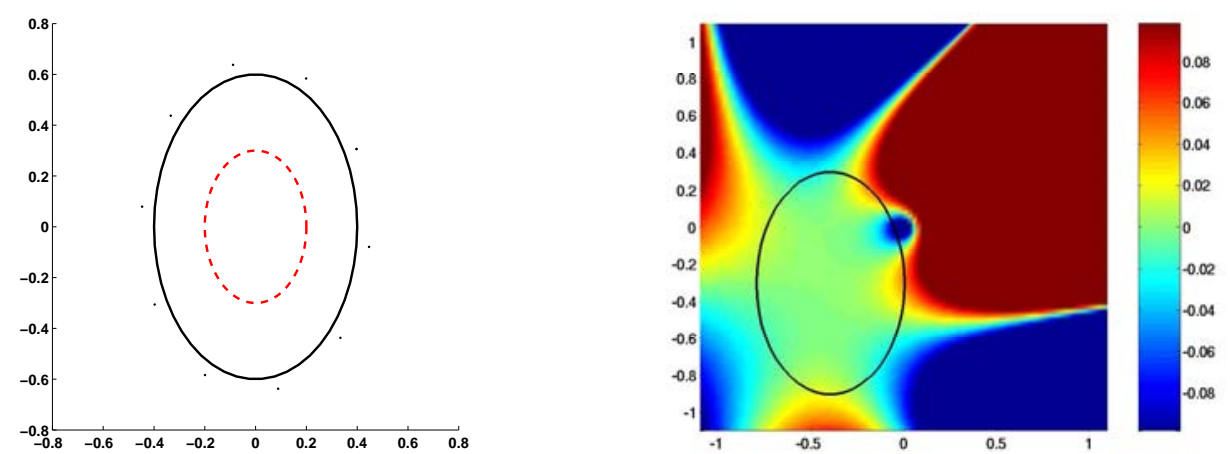

Fig. 6.9. The left figure shows the 10 different configurations for the approximation of the point source together with the dashed ellipsoidal obstacle. Each dot indicates the relative position of a source point $z_{0}^{(j)}$ with respect to its approximation domain $G\left(z_{0}^{(j)}\right)$ for $j=1, \ldots, 10$. The residual of the point source approximation is plotted in the right figure for one of these configurations.

The reconstruction of the kite and the boat will be done in a two-step fashion. First we use the strategy of translated circles to obtain a first guess of the shape of the obstacle and then we choose a more suitable approx- 

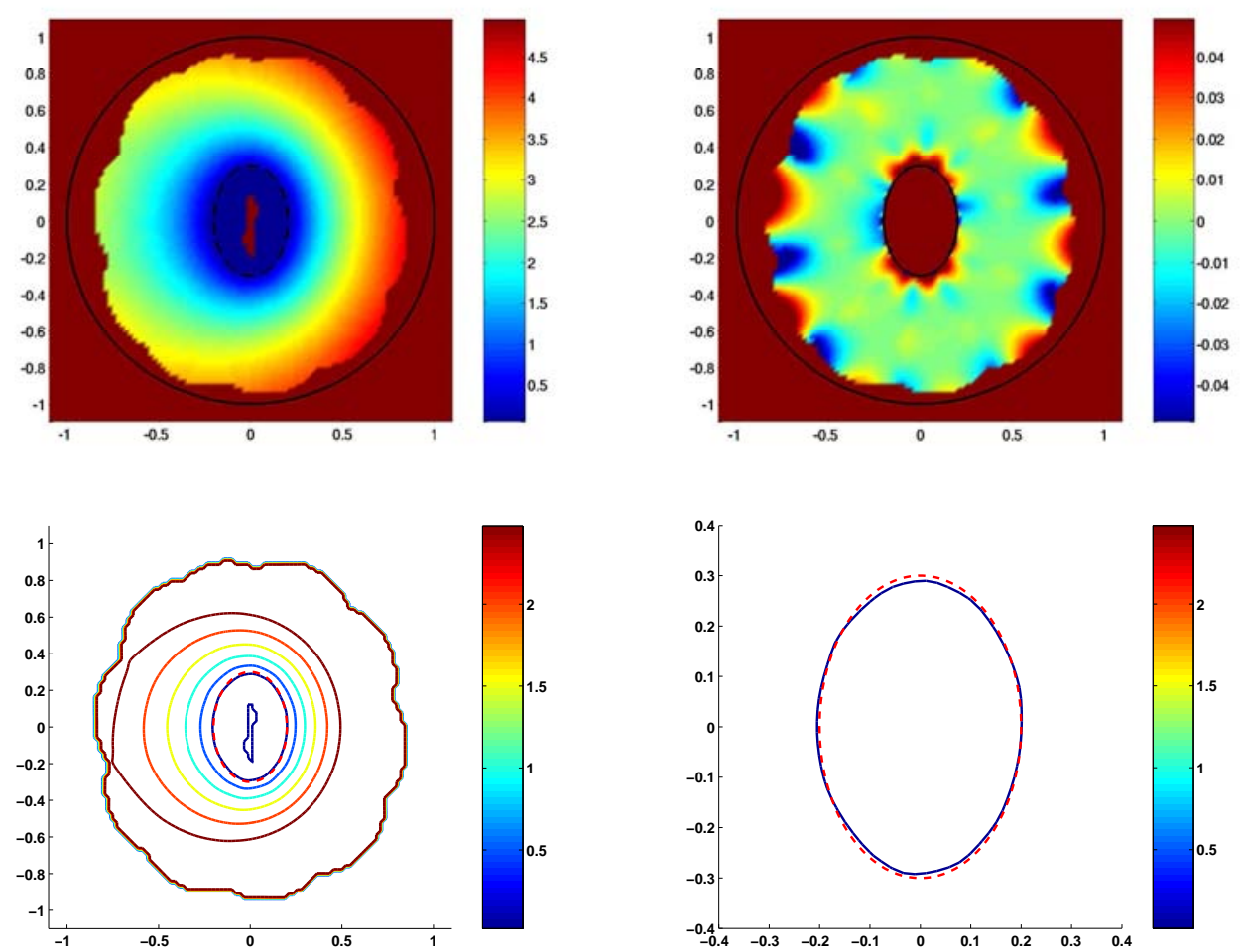

Fig. 6.10. The first shows the reconstructed field (left) together with the error (right) using the ellipsoidal approximation domains. In the second row the isolines of level 0, 0.5, 1, 1.5, 2, 2.5 as well as the final reconstruction of the obstacle's boundary are illustrated.

imation domain to find the boundary as the zero level set of the reconstructed field. Figure 6.11 illustrates the reconstruction with the circular approximation domains for the Settings 2 and 3. The parameter choice was $\tau=100, c=0.01$ and $\alpha=10^{-8}$ where we have used the 8 configurations $\left(0, G_{0}^{(j)}\right)$ of the previous example with a disk of radius $r=1$ in distance $\varepsilon_{r}=0.1$ of the source point. Obviously, the enlighted area $\mathcal{E}_{c}$ is not large enough to reconstruct the electric field up to the boundary of the obstacle with these general approximation domains and we would obtain a poor boundary reconstruction. Nevertheless, we obtain a first approximation to the obstacle's shape from the bounded component of the complement of the enlighted area $\mathcal{E}_{c}$. Therefore we are led to use adapted approximation domains that take care of the special geometry of the obstacle in the following. In a second run of the PSM we choose the reference configurations illustrated in Figure 6.12 for the reconstruction algorithm. Moreover, we speed up the algorithm by evaluating the field only in the region around the boundary of the obstacle. To this end we select to each source point $z_{0}^{(j)}$ shown in Figure 6.12 these points in $z \in \mathcal{S}$ with distance $\left|z-z_{0}^{(j)}\right|$ less than some predefined radius $r$. Then, for each reference configuration $\left(z_{0}^{(j)}, G\left(z_{0}^{(j)}\right)\right)$ the field is reconstructed in the disk $B_{r}\left(z_{0}^{(j)}\right)$. To combine all single reconstruc- 

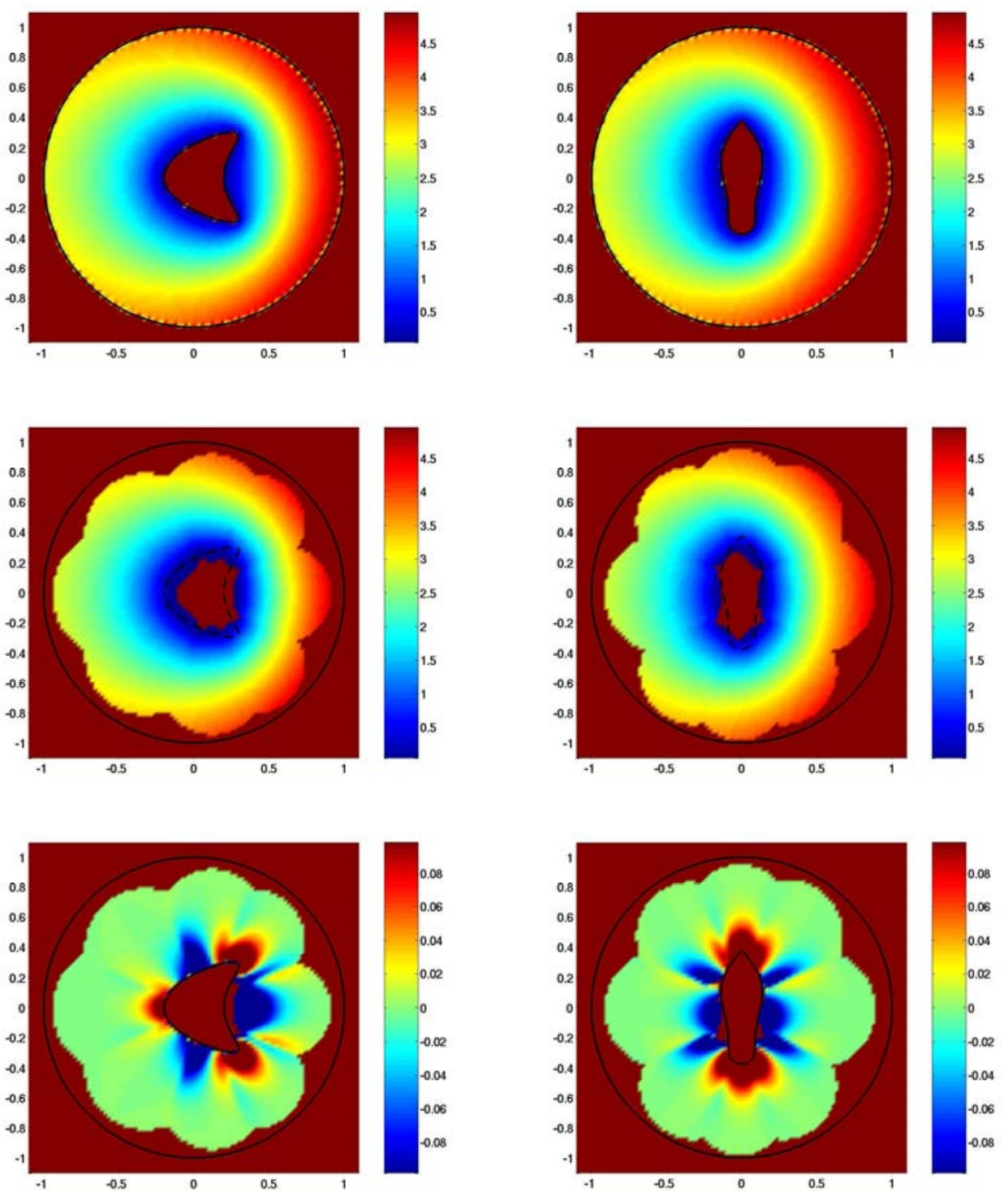

Fig. 6.11. The left column shows the numerical results for Setting 2 (kite) while the right one shows the results for Setting 3 (boat). The first row shows the calculated solution $u$ of the direct problem. The reconstructed field is illustrated in the second row and in the last row the difference of the true and reconstructed solution is plotted.

tions we again use an exponential weighting function by substituting $\omega_{\tau}^{(j)}$ with

$$
\omega_{\tau}^{(j)}(z)=\left\{\begin{array}{cl}
e^{-\tau\left\|z-z_{0}^{(j)}\right\|^{2}}, & z \in \mathcal{S} \cap B_{r}\left(z_{0}^{(j)}\right) \\
0 & , \quad z \in \mathcal{S} \backslash B_{r}\left(z_{0}^{(j)}\right)
\end{array},\right.
$$

and hence the complete reconstruction is given by (6.108) with the weights defined in (6.107). Note that in (6.109) the radii of the disks $B_{r}\left(z_{0}^{(j)}\right)$ do not depend on the translation direction in contrast to the previous definition (6.106). 

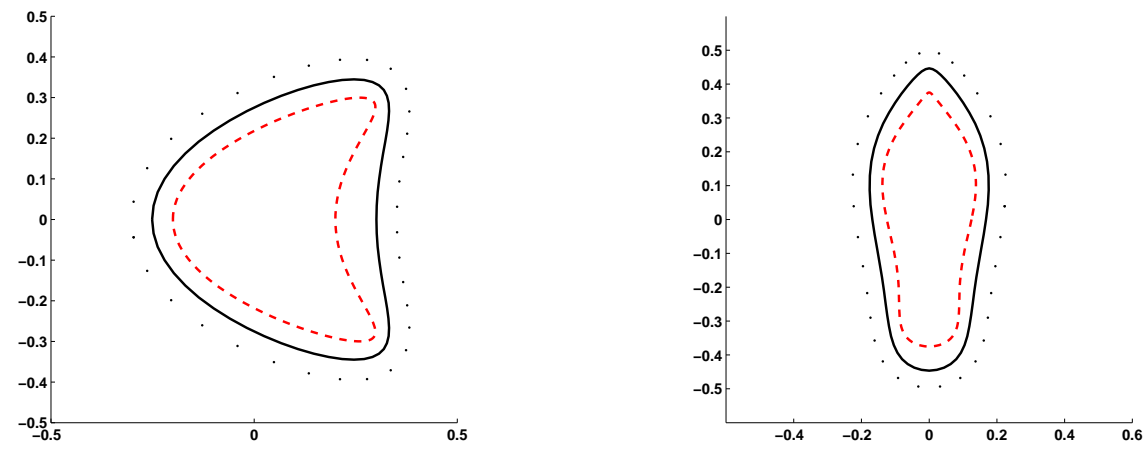

Fig. 6.12. The left figure shows the 32 different adapted configurations for the approximation of the point source together with the dashed obstacle of Setting 2. The adapted reference configurations for Setting 3 are plotted in the right figure. Each dot indicates the relative position of a source point $z_{0}^{(j)}$ with respect to its approximation domain $G\left(z_{0}^{(j)}\right)$ for $j=1, \ldots, 32$.

We summarize our numerical results using adapted approximation domains for the Settings 2 and 3 in Figure 6.13. Both reconstructions were computed with the parameters $r=0.3, \alpha=10^{-9}$ and $\tau=40$. A comparison of the error level shown in Figure 6.11 and Figure 6.13 proves that the adapted approximation domains yield a significant improvement in the reconstruction scheme. Using circular approximation domains only, a reconstruction of the boundary as presented in Figure 6.13 would not have been possible due to the nonconvex and non-isotropic geometry of the scatterers.

For reconstructing the solution to the Helmholtz boundary value problem from the Cauchy data on the measurement boundary $\partial \Omega$ we proceed as before. We only have to replace the static Herglotz wave operator by the Herglotz wave operator (5.45) itself and the static approximating functions by the Herglotz wave functions (5.44). Furthermore, we assume the a priori knowledge of some interior point $z_{0} \in D$ and apply strategy (5.109) for the choice of approximation domains in the first step of the reconstruction procedure. In our settings we may choose $z_{0}=0$ which slightly simplifies the notations. For each $z \in \mathcal{S}$ we choose $G(z)=\tilde{M}(z) G_{0}+z$ with the circular reference domain $G_{0}=\left\{(x, y):(x-1.1)^{2}+y^{2} \leq 1\right\}$ of the previous examples. Here, $\tilde{M}: \mathcal{S} \rightarrow S O(2, \mathbb{R})$ is a matrix-valued function that maps $z=\varrho e^{i \phi}$ to the matrix $M(\theta)$ given by (5.104), where the angle $\theta$ is the minimizer of $\left|\phi-\theta_{k}\right|$ among the sampling points $\theta_{k}$ of the unit circle. We illustrate this procedure with a simple example. We choose the uniform discretization

$$
\theta_{k}=\frac{k \pi}{4}, \quad k=0,1, \ldots, 7
$$

of the sphere $\mathbb{S}^{1}$ and calculate the approximation domain $G(z)$ of the point $z=0.5 e^{2.1 i}$ according to strategy (5.109). Then $\theta_{3}$ minimizes $\left|2.1-\theta_{k}\right|$ and we obtain $G(z)=M\left(\theta_{3}\right) G_{0}+z$ with the rotation matrix 

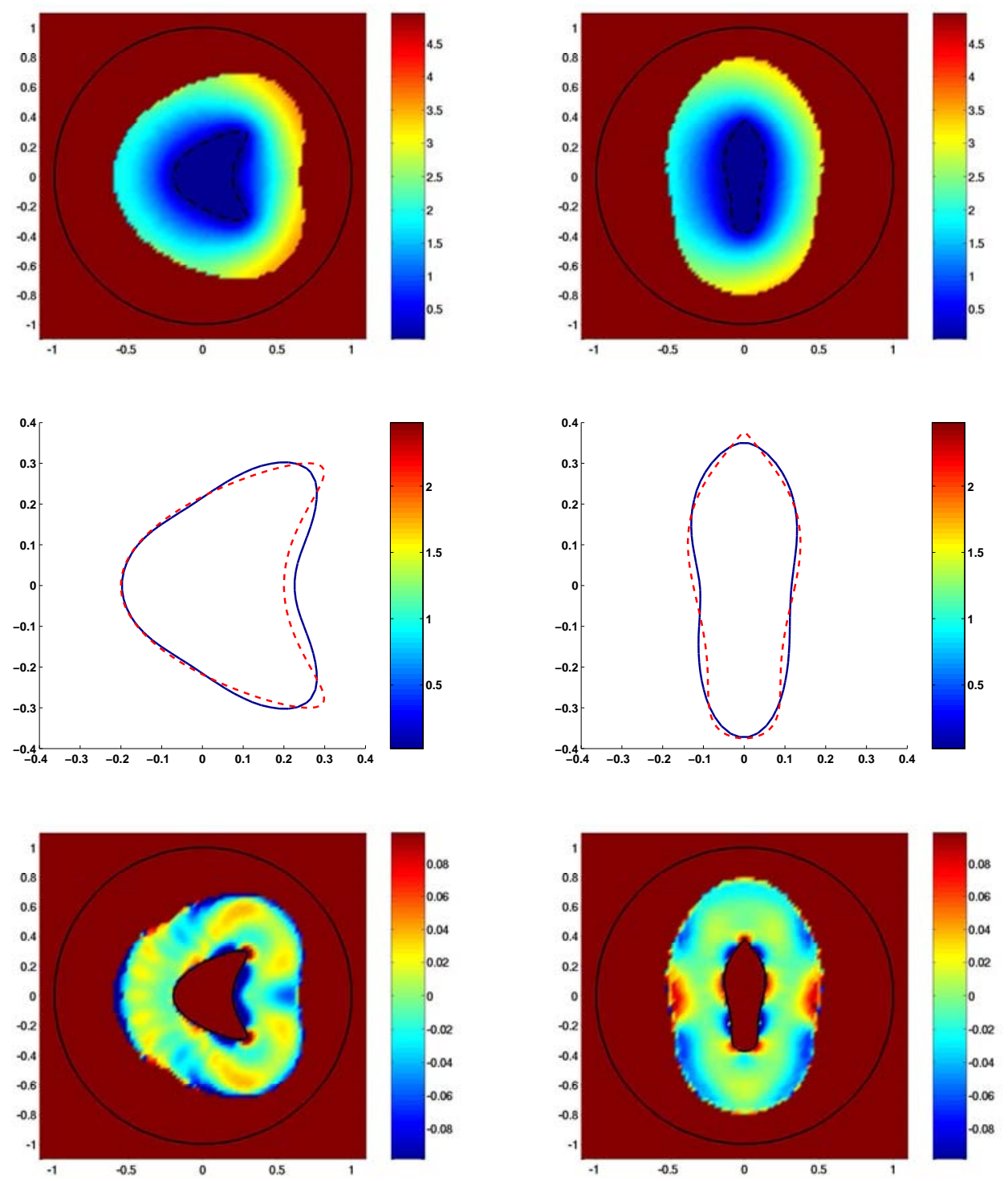

Fig. 6.13. The left column shows the numerical results for Setting 2 (kite) while the right one shows the results for Setting 3 (boat). The first row shows the reconstructed field in a neighbourhood of the obstacle. The boundary reconstruction is illustrated in the second row and in the last row the difference between the true and reconstructed solution is plotted.

$$
M\left(\theta_{3}\right)=\left(\begin{array}{rr}
-\cos \theta_{3} & \sin \theta_{3} \\
-\sin \theta_{3} & -\cos \theta_{3}
\end{array}\right)=\frac{\sqrt{2}}{2}\left(\begin{array}{rr}
1 & 1 \\
-1 & 1
\end{array}\right)
$$

which describes a rotation with angle $-\frac{\pi}{4}$. Figure 6.14 illustrates this procedure. This strategy moves the reference configuration $\left(0, G_{0}\right)$ first to the source point $z$ and then rotates the resulting configuration clockwise around $z$ by the minimizing angle. Therefore we obtain an approximation domain that is attached at $z$ approximately in the direction of the interior point $z_{0}$ of the scatterer. 

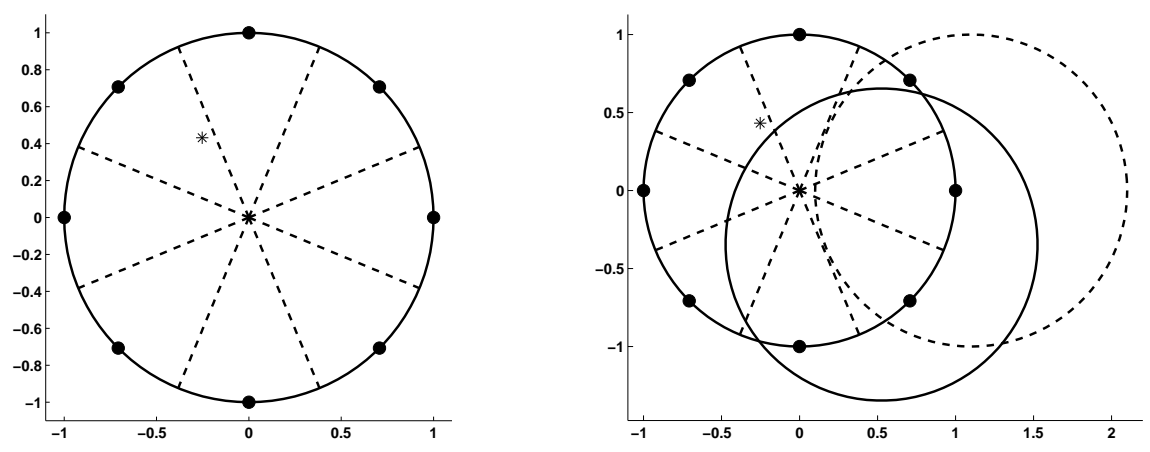

Fig. 6.14. The left figure shows the sampling points $\theta_{k}$ on the unit circle, the source point $z$ as well as the sectors on which the function $\tilde{M}$ is constant. The dashed reference domain $G_{0}$ is plotted together with the approximation domain $G(z)$ in the right picture.

In this situation we can apply Theorem 5.12 to calculate the densities $g_{z, \varepsilon}$ for all $z \in \mathcal{S}$, which results in (5.112) after discretization. Hence, the rotation $M(z)^{t} d$ of the argument in Theorem 5.12 can be performed by a circular shift of the density vector $g_{0, \varepsilon}$. In the following numerical examples we choose 50 different sampling points on the unit circle which provide an almost smooth variation of the translation directions across the 50 sectors. Hence we also do not have to apply the masking and weighting operations to obtain a smooth reconstruction and we reconstruct the field in one step completely with this choice of the approximation domains. It remains to calculate an approximation for the admissibility region $\mathcal{A}$. To this end we reconstruct the field twice with different regularization parameters $\alpha_{1}=10^{-8}, \alpha_{2}=2 \alpha_{1}$ and obtain

$$
\mathcal{E}_{c}=\left\{z:\left|\mathcal{I}_{\text {enl }}(z)\right|<c\right\}
$$

where the indicator function $\mathcal{I}_{\text {enl }}$ is given as the difference of the two reconstructions and $c=0.01$ is a cut-off parameter.

The boundary data on $\partial \Omega$ was chosen to be the total field of a scattering experiment with an incident superposition

$$
u^{i}(x)=\sum_{k=1}^{3} e^{i \kappa x \cdot d_{k}}, \quad x \in \mathbb{R}^{2},
$$

of plane waves with directions $d_{1}=(-1,0), d_{2}=1 / \sqrt{2}(1,1)$ and $d_{3}=$ $1 / \sqrt{2}(1,-1)$. The input data was generated by solving the direct scattering problem which will be explained in more detail when we present reconstructions for the threedimensional scattering problem. With the wave number $\kappa=3$ and 50 directions on the unit circle we obtain the reconstructions illustrated in Figure 6.15 to 6.20 where we have used the circular approximation domains with $\alpha=10^{-8}$ in the first reconstruction step.

The indicator function $\mathcal{I}_{\text {enl }}$ which is illustrated in Figure 6.16 together with 

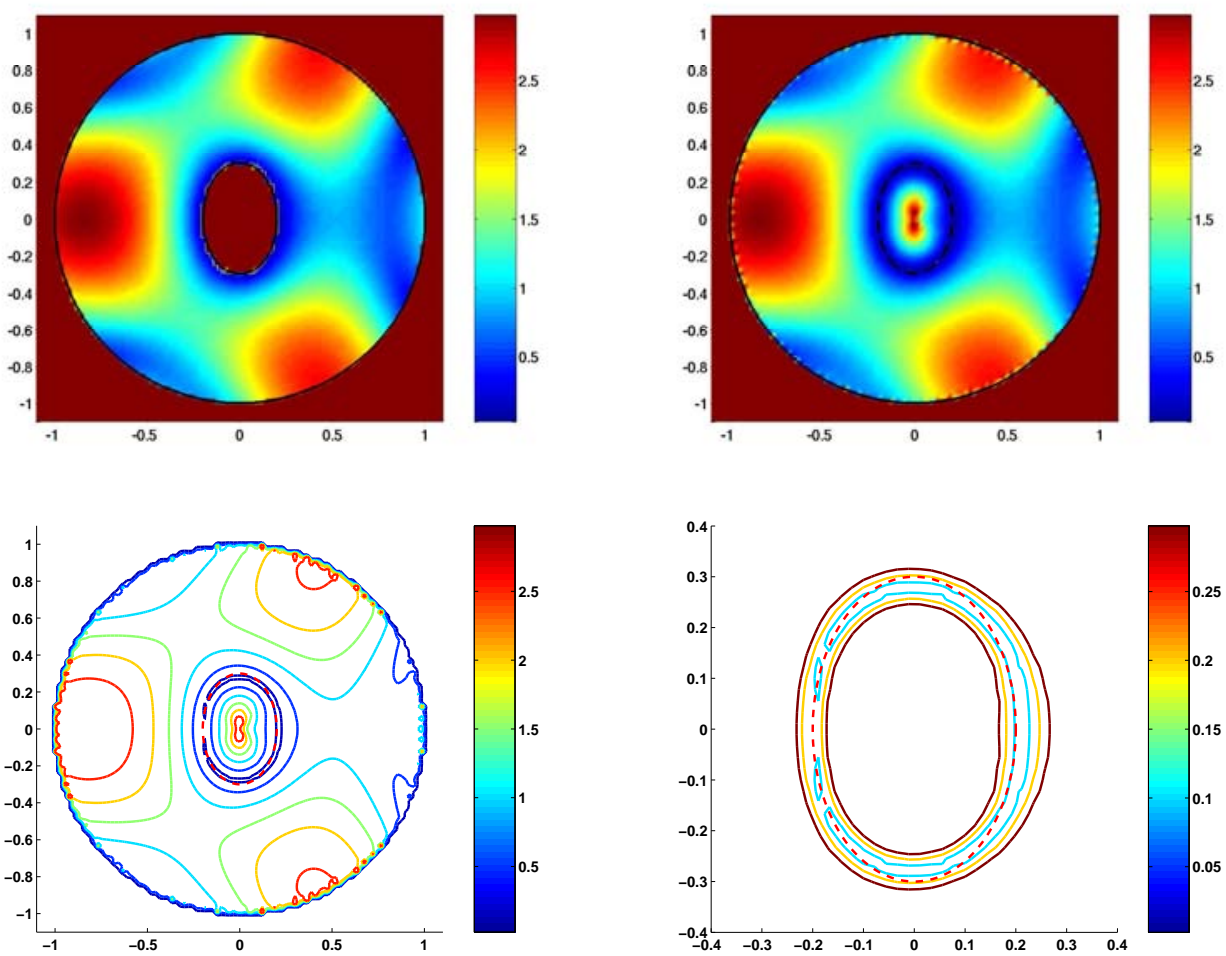

Fig. 6.15. The first row shows the modulus of the calculated solution $u$ of the direct problem (left) and the modulus of the reconstructed field (right) for the ellipse with wave number $\kappa=3$. The first figure of the second row illustrates the level sets $0.1,0.5,1,1.5,2,2.5$ while the second figure shows the level sets $0.1,0.2$ and 0.3 in more detail. The obstacle is indicated with a dashed line.
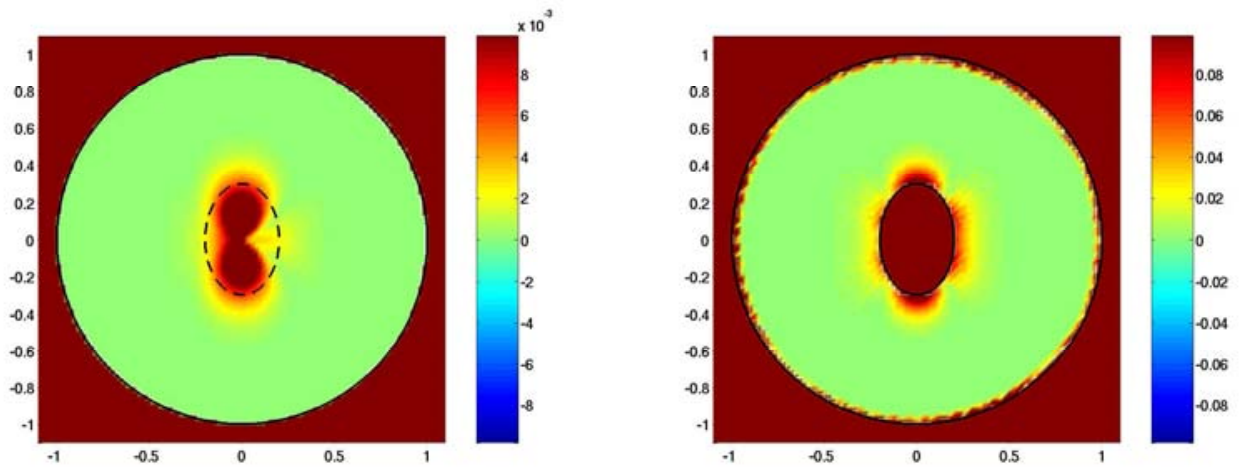

Fig. 6.16. The left figure shows the indicator function $\mathcal{I}_{\text {enl }}$ for the reconstruction of the ellipse with circular approximation domains. The right figure shows the error between the true and the reconstructed solution. 

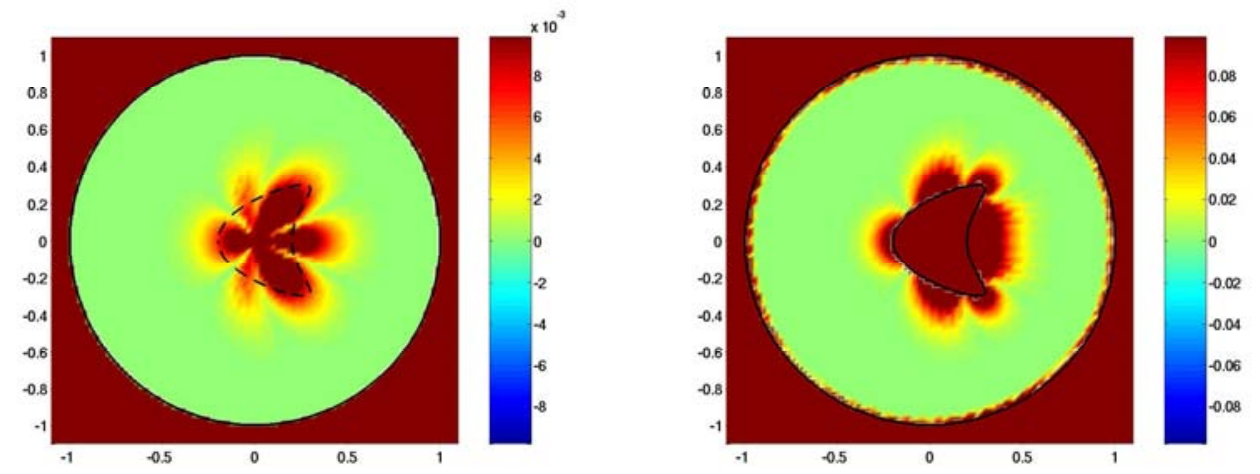

Fig. 6.17. The left figure shows the indicator function $\mathcal{I}_{\text {enl }}$ for the reconstruction of the kite with circular approximation domains. The right figure shows the error between the true and the reconstructed solution in this case.
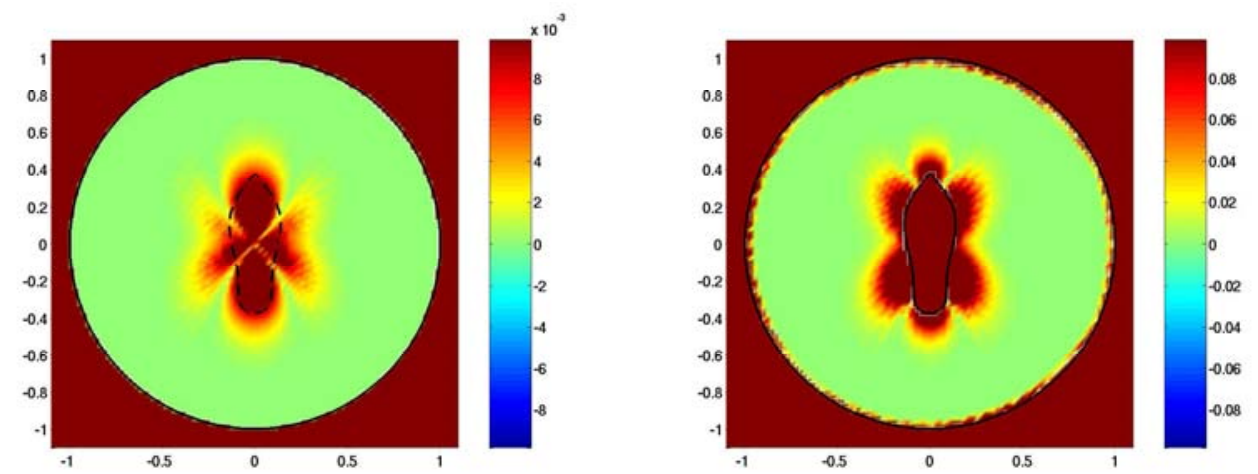

Fig. 6.18. The left figure shows the indicator function $\mathcal{I}_{\text {enl }}$ for the reconstruction of the boat with circular approximation domains. The right figure shows the error between the true and the reconstructed solution in this case.

the reconstruction error justifies to stop the reconstruction procedure for Setting 1 after the first step. Hence, the ellipse can be reconstructed using circular approximation domains only. The reconstruction of the kite with circular approximation domains yields an indicator function that blows off near the nonconvex part of the obstacle but is small near the convex part of the obstacle, see Figure 6.17. Similarly, the indicator function for the reconstruction of the boat illustrated in Figure 6.18 tells us not to trust the reconstructed values near the middle of the long sides of the boat.

Therefore we apply a second run of the point source method in these cases where we approximate the point source on the adapted domains of Figure 6.12. With the parameters $\alpha=10^{-7}, \tau=40$ and $r=0.3$ we obtain the reconstructions which are summarized in Figure 6.19 for the kite and in Figure 6.20 for the boat. Both obstacles can be reconstructed quite accurate with this two step strategy. While the first step yields a good approximation to the shape of the unknown obstacle the second step improves the quality of 

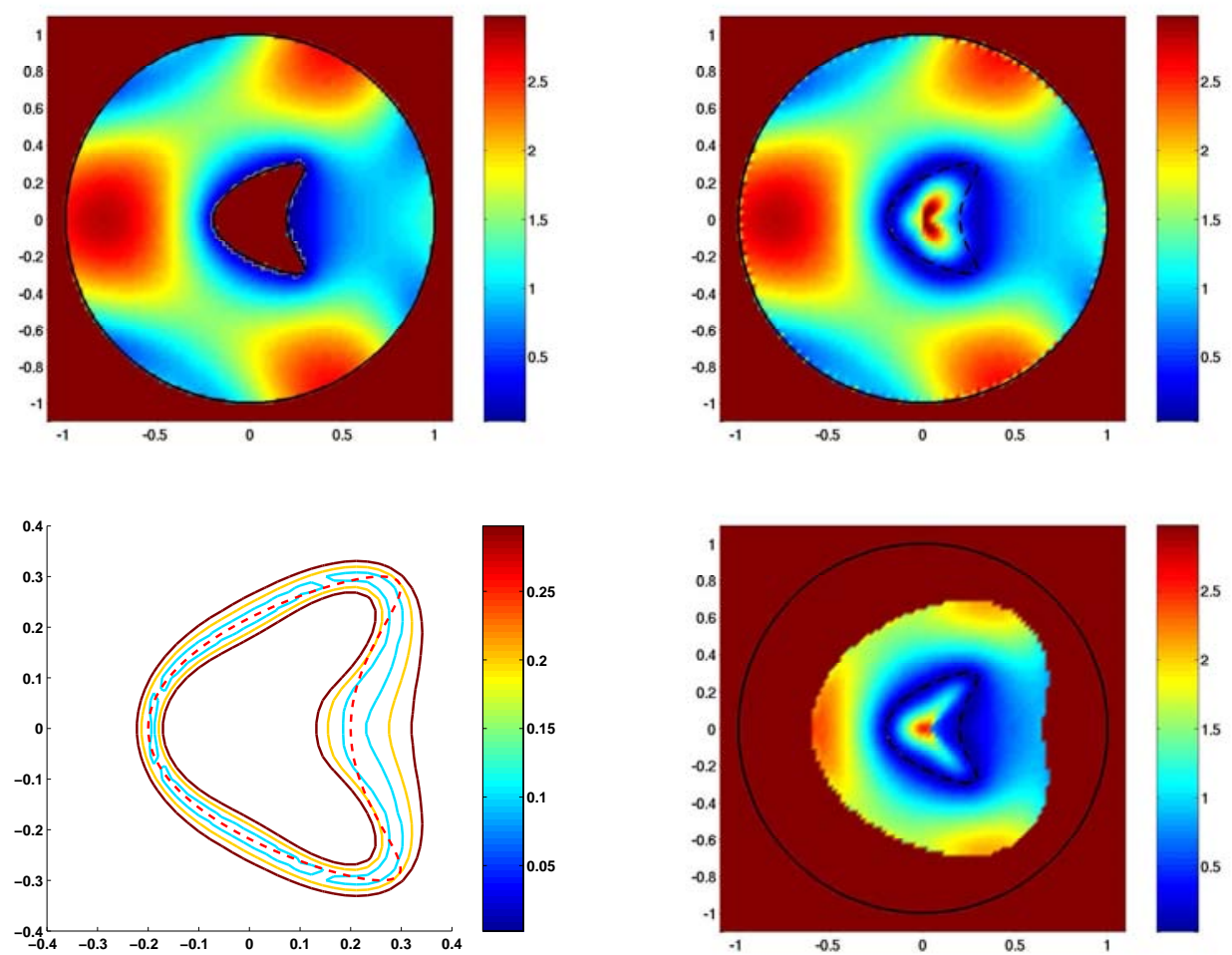

Fig. 6.19. The first row shows the modulus of the calculated solution $u$ of the direct problem (left) and the modulus of the reconstructed field (right) for the kite with wave number $\kappa=3$ where we used circular approximation domains. The reconstructions in the second row were produced with the adapted approximation domains of Figure 6.12. The first figure of the second row shows the level sets $0.1,0.2$ and 0.3 in more detail while the second figure illustrates the complete reconstructed field. The obstacle is indicated with a dashed line.

the reconstruction in the problematic regions which have been identified by the indicator function in the first step.

We close this chapter with the reconstruction of some sound-soft obstacles in the inverse scattering setting, where we use the far field pattern as input data for the reconstruction scheme. To produce this data we first have to solve the forward problem of calculating the scattered field $u^{s}$ due to the scattering of an incident field $u^{i}$ by the obstacle $D$. We have already seen in the previous section that the solution to this problem can be expressed in terms of a combined double- and single-layer potential

$$
u^{s}(x)=\int_{\partial D}\left(\frac{\partial \Phi(x, y)}{\partial \nu(y)}-i \eta \Phi(x, y)\right) \varphi(y) d s(y), \quad x \in \mathbb{R}^{m} \backslash \bar{D}
$$

on the boundary $\partial D$ with a continuous density $\varphi \in C(\partial D)$ and a positive coupling parameter $\eta>0$, which we choose as $\eta=\kappa$. By the boundary condition $u=u^{i}+u^{s}=0$ on $\partial D$ we have to solve the integral equation

$$
\frac{1}{2} \varphi(x)+\int_{\partial D}\left(\frac{\partial \Phi(x, y)}{\partial \nu(y)}-i \eta \Phi(x, y)\right) \varphi(y) d s(y)=-u^{i}(x)
$$



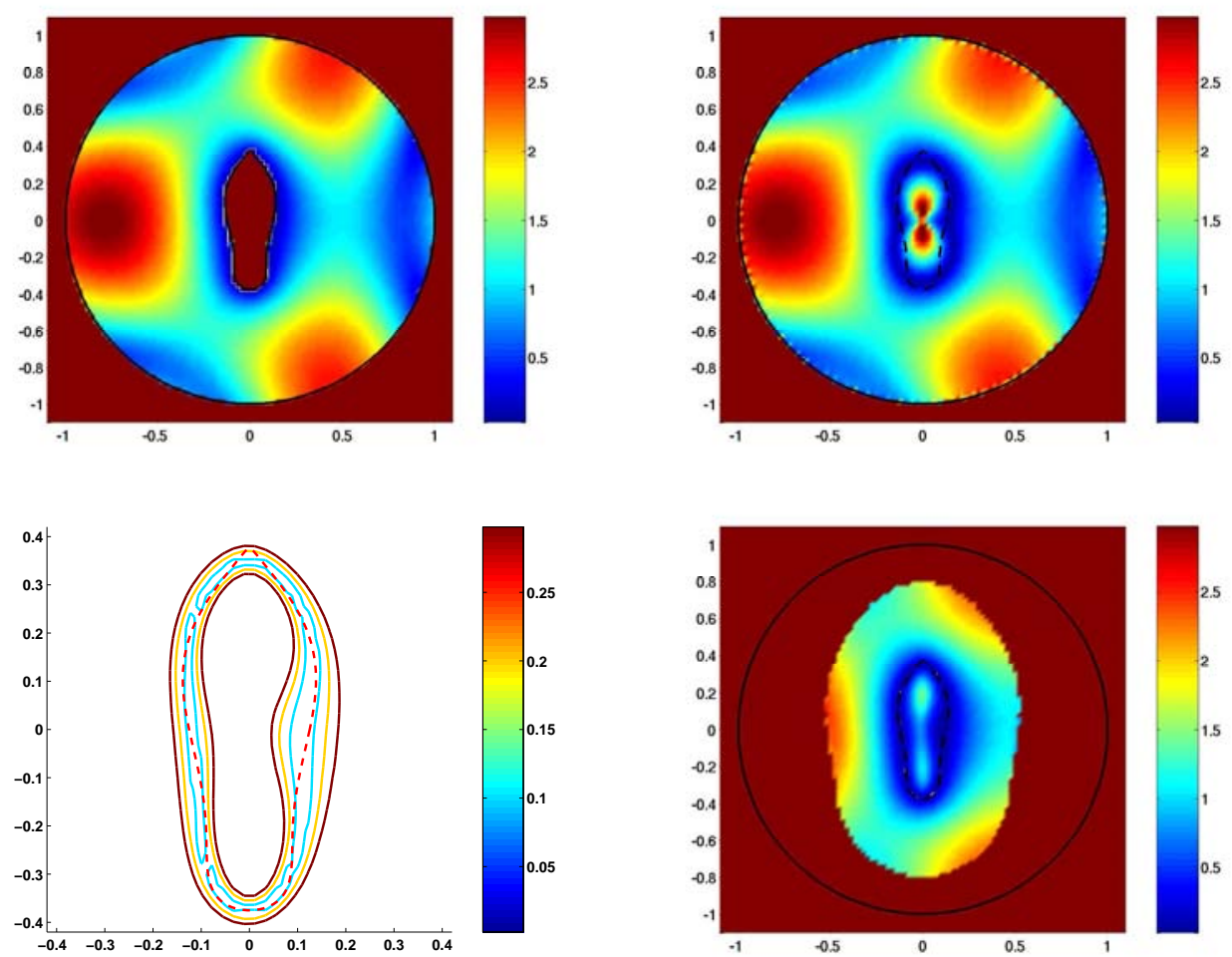

Fig. 6.20. The first row shows the modulus of the calculated solution $u$ of the direct problem (left) and the modulus of the reconstructed field (right) for the boat with wave number $\kappa=3$ where we used circular approximation domains. The reconstructions in the second row were produced with the adapted approximation domains of Figure 6.12. The first figure of the second row shows the level sets $0.1,0.2$ and 0.3 in more detail while the second figure illustrates the complete reconstructed field. The obstacle is indicated with a dashed line.

of the second kind on $\partial D$ to calculate the density $\varphi$ in the ansatz function, when we assume the unit normal $\nu$ to be orientated into the exterior of the scatterer $D$.

To avoid repetitions we restrict ourselves to the three-dimensional case. Since the separation of the logarithmic singularity as demonstrated in Examples 4.2 to 4.7 can not be carried over to the three-dimensional setting in a straightforward way, we just ignore the occurring weak singularities of the kernels by introducing a cut-off scheme. Hence, we use the modified kernel

$$
\tilde{k}(x, y)=\left\{\begin{array}{cl}
k(x, y), & \text { for }|x-y|>c \\
0, & \text { for }|x-y| \leq c
\end{array}\right.
$$

with the cut-off parameter $c>0$ for the kernel

$$
k(x, y)=2\left(\frac{\partial \Phi(x, y)}{\partial \nu(y)}-i \eta \Phi(x, y)\right)
$$

of the combined layer operator. For a more accurate quadrature of these kernels we refer the reader to the thesis [77] of Wienert. 
The sound-soft scatterer is given by one of the obstacles illustrated in Figure 6.21. The first obstacle is a ball of radius $r=0.5$, the second obstacle is a
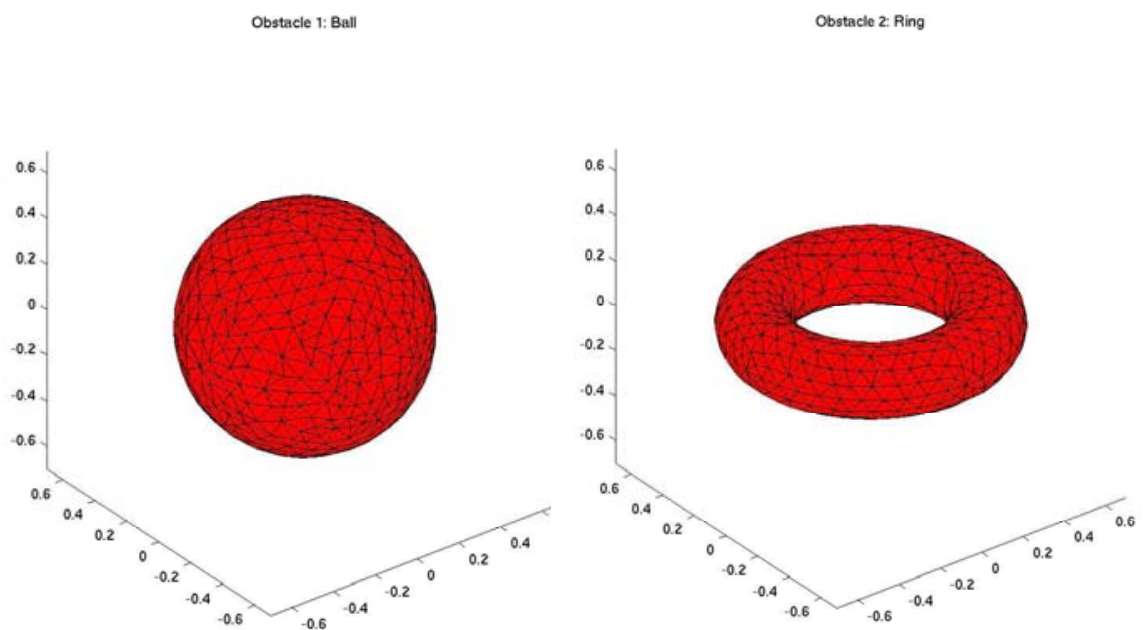

Fig. 6.21. The sound-soft scatterers in the numerical examples are a ball (left) with 1170 triangles and a ring (right) with 1184 triangles for the surface mesh.

ring with inner radius $r_{i}=0.3$ and outer radius $r_{o}=0.6$, i.e. the ring is given by the set of points

$$
\left\{(x, y, z) \in \mathbb{R}^{3}:\left(c-\sqrt{x^{2}+y^{2}}\right)^{2}+z^{2} \leq a^{2}\right\}
$$

with the center radius $c=\frac{1}{2}\left(r_{i}+r_{o}\right)$ and the radius $a=\frac{1}{2}\left(r_{o}-r_{i}\right)$ of the tube. The surface of the ball and the torus is discretized with a mesh of $N=1170$ and $N=1184$ triangles, respectively. For the implementation of the integral operators we apply the midpoint rule and approximate the integral operator

$$
(A \varphi)(x)=\int_{\partial D} k(x, y) \varphi(y) d s(y)
$$

with

$$
\left(A_{N} \varphi\right)(x)=\sum_{l=1}^{N} \tilde{k}\left(x, y_{l}\right) \varphi\left(y_{l}\right) h_{l},
$$

where $y_{l}$ is the barycenter and $h_{l}$ is the area of the $l$-th triangle in the mesh. Notice that the barycenters $y_{l}$ do not lie on the boundary $\partial D$. Since we solve the integral equation (6.115) with Nyström's method we have to keep in mind that we do not integrate over the boundary $\partial D$ of the scatterer but over some approximant surface $\partial \tilde{D}$. The validity of this approximation is justified by the continuous dependence of the scattered field from the boundary $\partial D$, see Theorem 5.7 in [7]. Hence, solving the linear system of equations 


$$
\varphi_{l}-\sum_{m=1}^{N} h_{m} \tilde{k}\left(y_{l}, y_{m}\right) \varphi_{m}=-2 u^{i}\left(y_{l}\right), \quad l=1, \ldots, N
$$

for the coefficients $\varphi_{l}$ provides the approximate solution

$$
\varphi_{N}(x)=-2 u^{i}(x)+\sum_{m=1}^{N} h_{m} \tilde{k}\left(x, y_{m}\right) \varphi_{m}, \quad x \in \partial D
$$

of the integral equation (6.115). Thus we compute an approximation of the far field pattern of the scattered wave by inserting (6.122) into the discretized far field operator given by

$$
\left(A_{N}^{\infty} \varphi\right)(d)=\sum_{m=1}^{N} k^{\infty}\left(d, y_{m}\right) \varphi\left(y_{m}\right) h_{m}, \quad d \in \mathbb{S}^{2}
$$

with the kernel

$$
k^{\infty}(d, y)=\frac{1}{4 \pi}\left(\frac{\partial}{\partial \nu(y)} e^{-i \kappa y \cdot d}-i \eta e^{-i \kappa y \cdot d}\right), \quad d \in \mathbb{S}^{2}, y \in \partial D .
$$

The latter can easily be deduced from the asymptotics

$$
\frac{e^{i \kappa|x-y|}}{|x-y|}=\frac{e^{i \kappa|x|}}{|x|}\left(e^{-i \kappa \hat{x} \cdot y}+O\left(\frac{1}{|x|}\right)\right)
$$

and

$$
\frac{\partial}{\partial \nu(y)} \frac{e^{i \kappa|x-y|}}{|x-y|}=\frac{e^{i \kappa|x|}}{|x|}\left(\frac{\partial e^{-i \kappa \hat{x} \cdot y}}{\partial \nu(y)}+O\left(\frac{1}{|x|}\right)\right)
$$

of the fundamental solution and the definition (6.69) of the far field pattern. To check the quality of the forward solver we calculate the far field pattern of the point source $\Phi\left(\cdot, z_{b}\right)$ and $\Phi\left(\cdot, z_{r}\right)$ for the ball and the ring, respectively. Here $z_{b}=(0,0,0)$ and $z_{r}=(0.45,0,0)$ are source points inside the scattering obstacle and we substitute the right hand side of (6.115) with two times the values of the source point on $\partial D$, namely with $\left.2 \Phi(\cdot, z)\right|_{\partial D}$ and $z=z_{b}, z_{r}$. Solving the resulting integral equation with Nyström's method and applying the far field operator $A_{N}^{\infty}$ to this solution we obtain an approximation $\tilde{\Phi}^{\infty}$ of the far field pattern of the interior point source. Then we compare the calculated with the known far field pattern

$$
\Phi^{\infty}(d, z)=\frac{1}{4 \pi} e^{-i \kappa d \cdot z}, \quad z=z_{b}, z_{r}, \quad d \in \mathbb{S}^{2}
$$

of the point source. Table 6.1 and 6.2 show both the $L^{2}$-error and the error in the maximum norm for several numbers of triangles in the surface mesh of the scatterers and the wave number $\kappa=2$. 


\begin{tabular}{ccc}
\hline \hline$N$ & $\left\|\Phi^{\infty}\left(\cdot, z_{b}\right)-\tilde{\Phi}^{\infty}\left(\cdot, z_{b}\right)\right\|_{L^{2}}$ & $\left\|\Phi^{\infty}\left(\cdot, z_{b}\right)-\tilde{\Phi}^{\infty}\left(\cdot, z_{b}\right)\right\|_{\infty}$ \\
\hline 100 & 0.1755 & 0.004711 \\
174 & 0.1490 & 0.003377 \\
374 & 0.1031 & 0.002339 \\
580 & 0.0844 & 0.001898 \\
1170 & 0.0626 & 0.001405 \\
\hline \hline
\end{tabular}

Table 6.1. The table illustrates the error between the true far field pattern $\Phi^{\infty}$ and its approximation $\tilde{\Phi}^{\infty}$ on the sphere $\mathbb{S}^{2}$ which is discretized with 174 triangles. The error is shown for several numbers $N$ of triangles in the mesh of the ball.

\begin{tabular}{ccc}
\hline \hline$N$ & $\left\|\Phi^{\infty}\left(\cdot, z_{r}\right)-\tilde{\Phi}^{\infty}\left(\cdot, z_{r}\right)\right\|_{L^{2}}$ & $\left\|\Phi^{\infty}\left(\cdot, z_{r}\right)-\tilde{\Phi}^{\infty}\left(\cdot, z_{r}\right)\right\|_{\infty}$ \\
\hline 424 & 0.1482 & 0.004284 \\
696 & 0.1429 & 0.004111 \\
1184 & 0.1048 & 0.003001 \\
1472 & 0.1092 & 0.003152 \\
\hline \hline
\end{tabular}

Table 6.2. The table illustrates the error between the true far field pattern $\Phi^{\infty}$ and its approximation $\tilde{\Phi}^{\infty}$ on the sphere $\mathbb{S}^{2}$ which is discretized with 174 triangles. The error is shown for several numbers $N$ of triangles in the mesh of the ring.

For the calculation of the density $g_{0}$ we discretize the Herglotz wave operator and solve the regularized integral equation (5.89) in the same way as we did for the integral equation $(6.115)$ on the boundary $\partial D$. This procedure provides an approximation of the fundamental solution $\Phi(\cdot, 0)$ on the approximation domain $G_{0}$ in terms of a Herglotz wave function. For the reference domain we choose a ball of radius $r=1$ the center of which is located in the point $(1.1,0,0)$, i.e.

$$
G_{0}=\left\{(x, y, z) \in \mathbb{R}^{3}: \sqrt{(x-1.1)^{2}+y^{2}+z^{2}} \leq 1\right\} .
$$

In the three-dimensional setting we can not use the strategy (5.109) for the choice of the approximation domain without restricting ourselves to a triangulation of the unit sphere corresponding to the platonic bodies and a lot of tricky implementation work. Therefore we will use the strategy (5.115) of translated approximation domains as we did in the example of the Laplace equation. The approach of gaining a complete reconstruction from the single directional reconstructed fields can be performed analogously to (6.104), (6.105) and (6.108) by cutting some balls out of the enlighted area and averaging the restricted directional reconstructions as demonstrated exemplarily for the 2D Laplace equation. For the sake of simplicity we use the arithmetic mean for the averaging process, i.e. we choose $\tau=0$.

We consider the scattering of the plane wave $u^{i}(x, d)=e^{i \kappa x \cdot d}$ with direction $d=(0,1,0)$ of incidence and wave number $\kappa=2$ by the sound-soft ball. The reconstruction of the total field is illustrated in Figure 6.22, where the regularization parameters were chosen to be $\alpha_{1}=10^{-8}$ and $\alpha_{2}=2 \alpha_{1}$. For the reconstruction of the scattered field we used the 6 face normals $n_{j}$ of the unit cube to define the configurations $\left(0, G_{0}^{(j)}\right)$ given by 

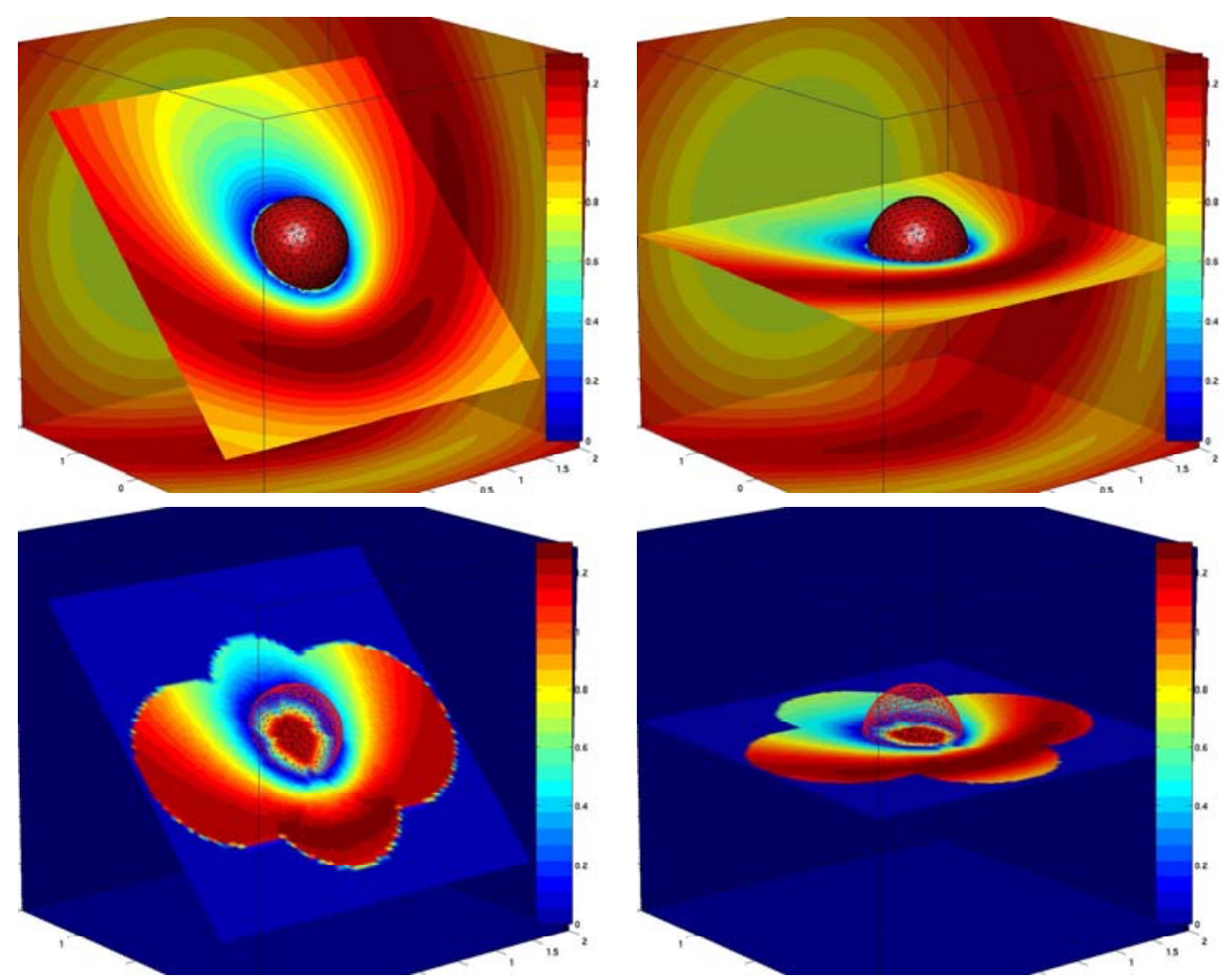

Fig. 6.22. The first row shows the modulus of the calculated total field $u$ of the direct problem while the second row illustrates the modulus of the reconstructed total field for the ball with wave number $\kappa=2$.

$$
G_{0}^{(j)}:=\mathbb{S}^{2}+1.1 n_{j}, \quad j=1, \ldots, 6 .
$$

For the determination of the enlighted area we worked with the cut-off value $c=0.15$ for each configuration and the control parameter $q=0.7$ for the construction of the balls $B_{r}\left(z_{0}^{(j)}\right)$. To the reconstructed scattered field we added the restriction of the incident field $u^{i}$ to the enlighted area to obtain the approximation $u_{\mathrm{rec}, \varepsilon}=u_{\mathrm{rec}, \varepsilon}^{s}+u^{i}$ of the total field.

From the latter we extracted an isosurface corresponding to the level 0.1 to gain an approximation of the scatterer. Figure 6.23 shows the resulting reconstruction of the obstacle $D$. As we have already seen in the twodimensional case, the reconstruction is worse in the neighbourhood of the obstacle, but we can improve the quality of the reconstruction by adjusting the approximation domain $G_{0}$ to the scatterer geometry. Figure 6.24 shows the isosurface of level 0.1 , where we used the approximation domains

$$
G_{0}^{(j)}:=0.7 \mathbb{S}^{2}+0.75 n_{j}, \quad j=1, \ldots, 6 .
$$

to reconstruct the scattered field. A further improvement of the reconstruction is achieved when using additional configurations which is also illustrated in Figure 6.24. Here we additionally reconstruct the scattered field for the approximation domains 

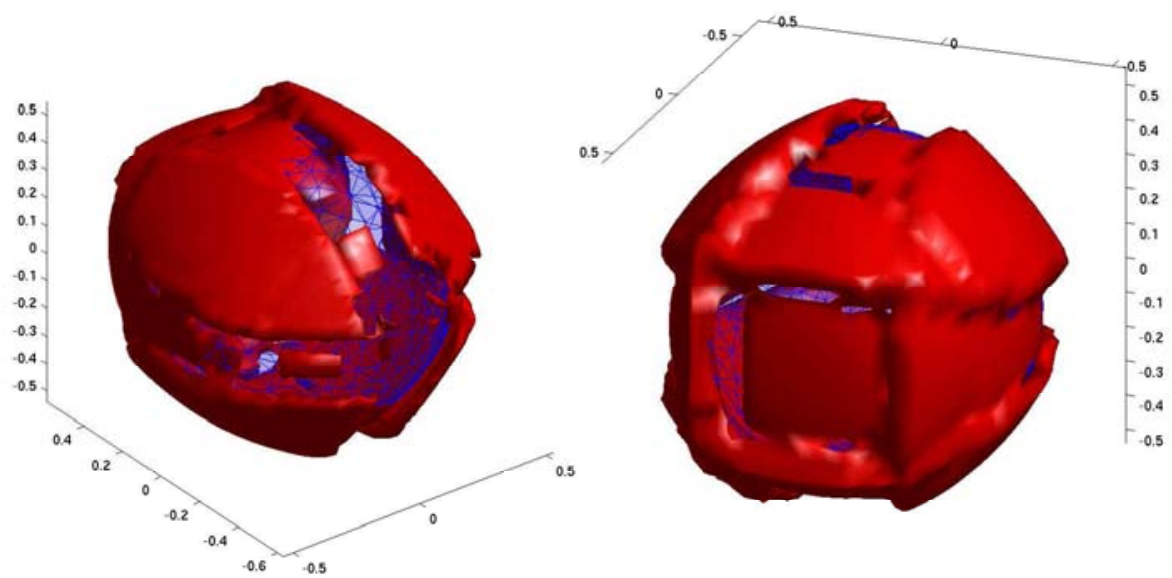

Fig. 6.23. Reconstruction of the sound-soft ball with 6 different configurations of the approximation domain. The reconstruction shows the isosurface of level 0.1 of the reconstructed total field. The obstacle $D$ is indicated with the blue mesh.

$$
G_{0}^{(j)}:=0.7 \mathbb{S}^{2}+0.75 n_{j}, \quad j=7, \ldots, 26,
$$

where $n_{j}, 7 \leq j \leq 26$ denote the 8 normalized diagonals of the cube and the 12 normalized diagonals of the cube's faces.

Let us now consider the scattering of the incident plane wave $u^{i}(x)=$ $e^{i \kappa x \cdot d}$ with direction $d=(0,1,0)$ and wave number $\kappa=2$ by the ring $(6.118)$. For the reconstruction of the total field we use the translation strategy with the configurations $\left(0, G_{0}^{(j)}\right)$ and the approximation domains

$$
G_{0}^{(j)}:=\mathbb{S}^{2}+1.1 n_{j}, \quad j=1, \ldots, 26,
$$

with the 26 directions $n_{j}$ of the previous example. The enlighted area is computed with the regularization parameters $\alpha_{1}=10^{-8}$ and $\alpha_{2}=2 \alpha_{1}$, the cut-off parameter $c=0.15$ and the control parameter $q=0.7$ of the balls $B_{r}\left(z_{0}^{(j)}\right)$. Both the total field and its reconstruction are illustrated in Figure 6.25. From this reconstructed total field we extracted the isosurface of level 0.1 for an approximation of the scattering obstacle, which is illustrated in Figure 6.26. Though we are able to detect the outer boundary of the ring, we fail to reconstruct the boundary of the ring's hole as well as its flat bottom and top part. This is due to the choice of the approximation domains which are not adjusted to the shape of the scatterer. By the nature of the PSM we can not expect to reconstruct neither the inside nor the bottom and top region of the ring since the sampling points in these parts do not fulfill the admissibility condition $\bar{D} \subset G(z)$ and do therefore not belong to the admissibility region.

Nevertheless we can improve the reconstruction by considering smaller approximation domains given by $(6.131)$ as illustrated in Figure 6.27. This Figure also shows reconstructions of the ring, where we used two further 

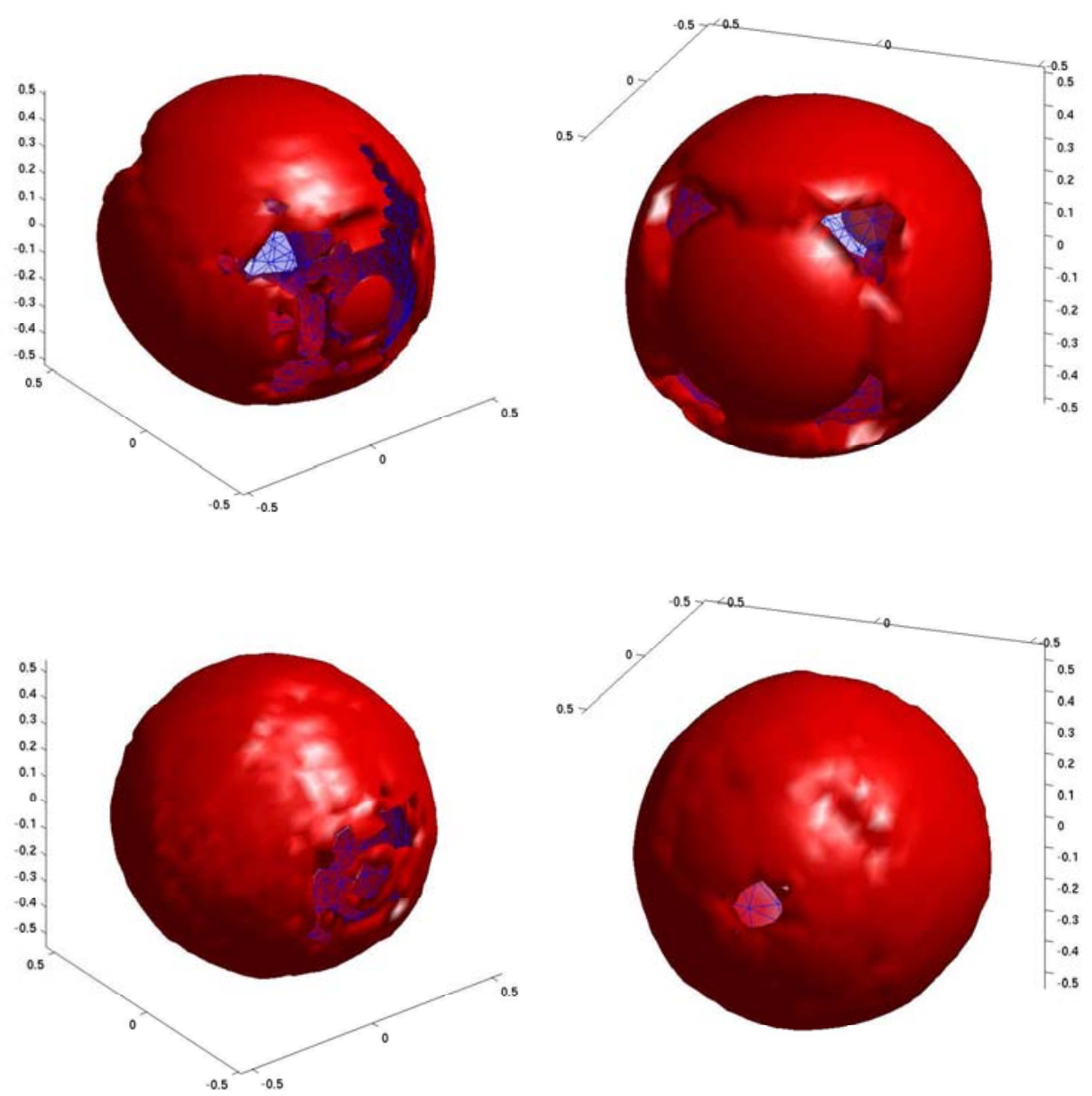

Fig. 6.24. The first row shows the isosurface of level 0.1 of the total field reconstructed with 6 configurations of the approximation domain according to the face normals of the unit cube. In contrast to Figure 6.23 the approximation domain was chosen to be a ball of radius $r=0.7$, comparable to the size of the scatterer. The second row shows the same isosurface where we used 26 different configurations.

incident fields with directions $d=(1,0,0)$ and $d=(0,0,1)$. In this case we reconstructed the total field for each incident wave in the first step, calculated the arithmetic mean of the reconstructed fields and, finally, extracted an appropriate isosurface. Although we get rid of the artefacts in the reconstruction and succeed in determining the outer boundary of the ring we still fail to reconstruct the inner part of the boundary.

Since the PSM is independent of the special type of incident field we may try to reconstruct the scatterer with an incident point source rather than with a plane wave. In particular, if we put a point source in the center $(0,0,0)$ of the ring, we expect the scattered field to be high on the interior parts and neglectable on the exterior parts of the boundary of the ring. With the scattering of plane waves we were faced with the opposite situation. 

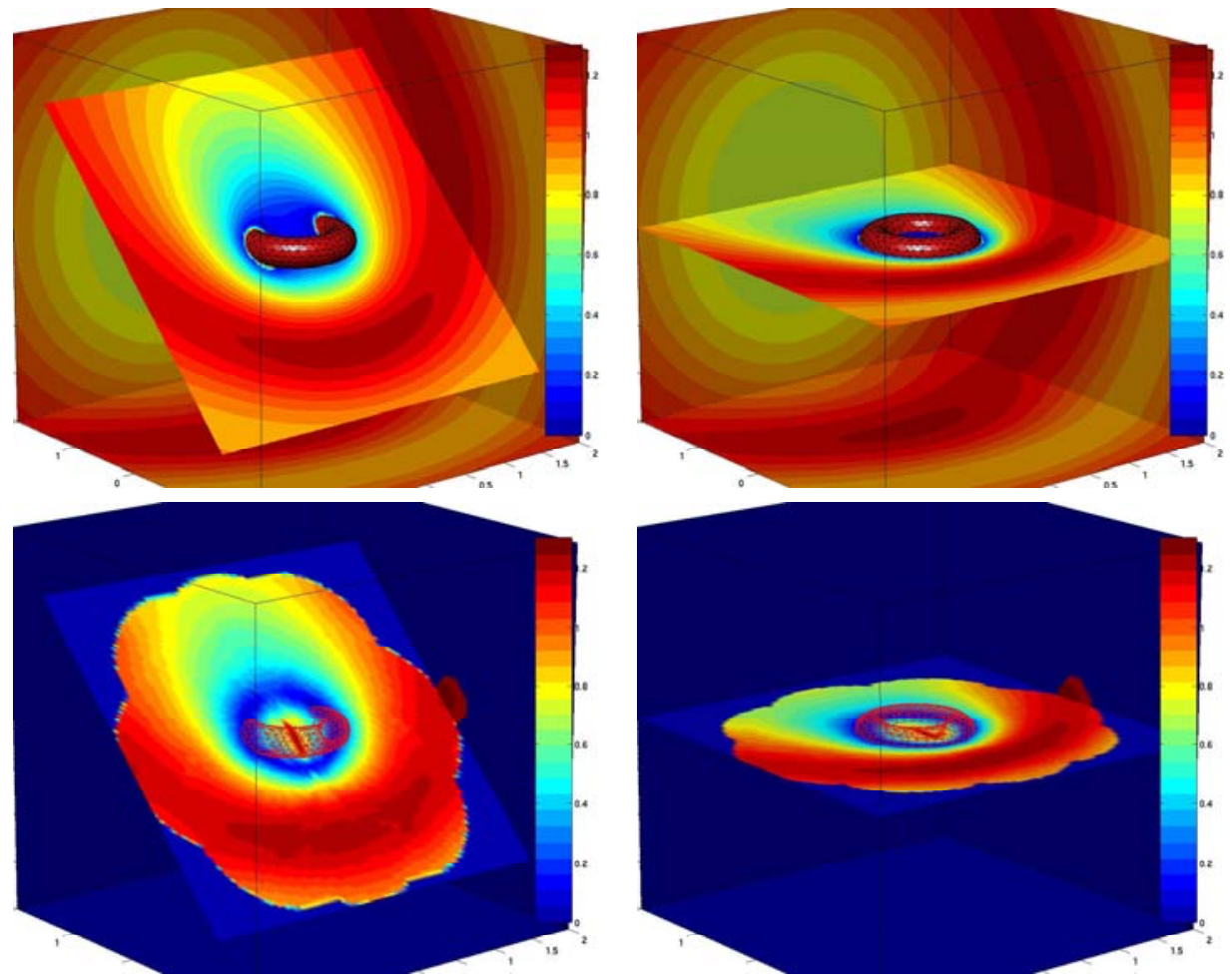

Fig. 6.25. The first row shows the modulus of the calculated total field $u$ of the direct problem while the second row illustrates the modulus of the reconstructed total field for the ring with wave number $\kappa=2$.
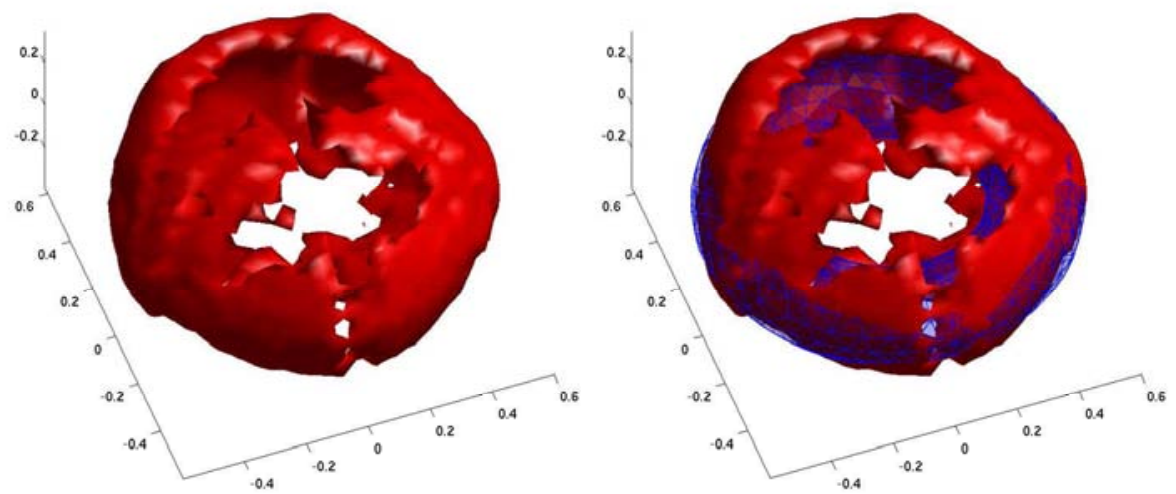

Fig. 6.26. Reconstrucion of the sound-soft ring with 26 different configurations of the approximation domain. The reconstruction shows the isosurface of level 0.1 of the reconstructed total field. The obstacle $D$ is indicated with a blue mesh in the right picture. 

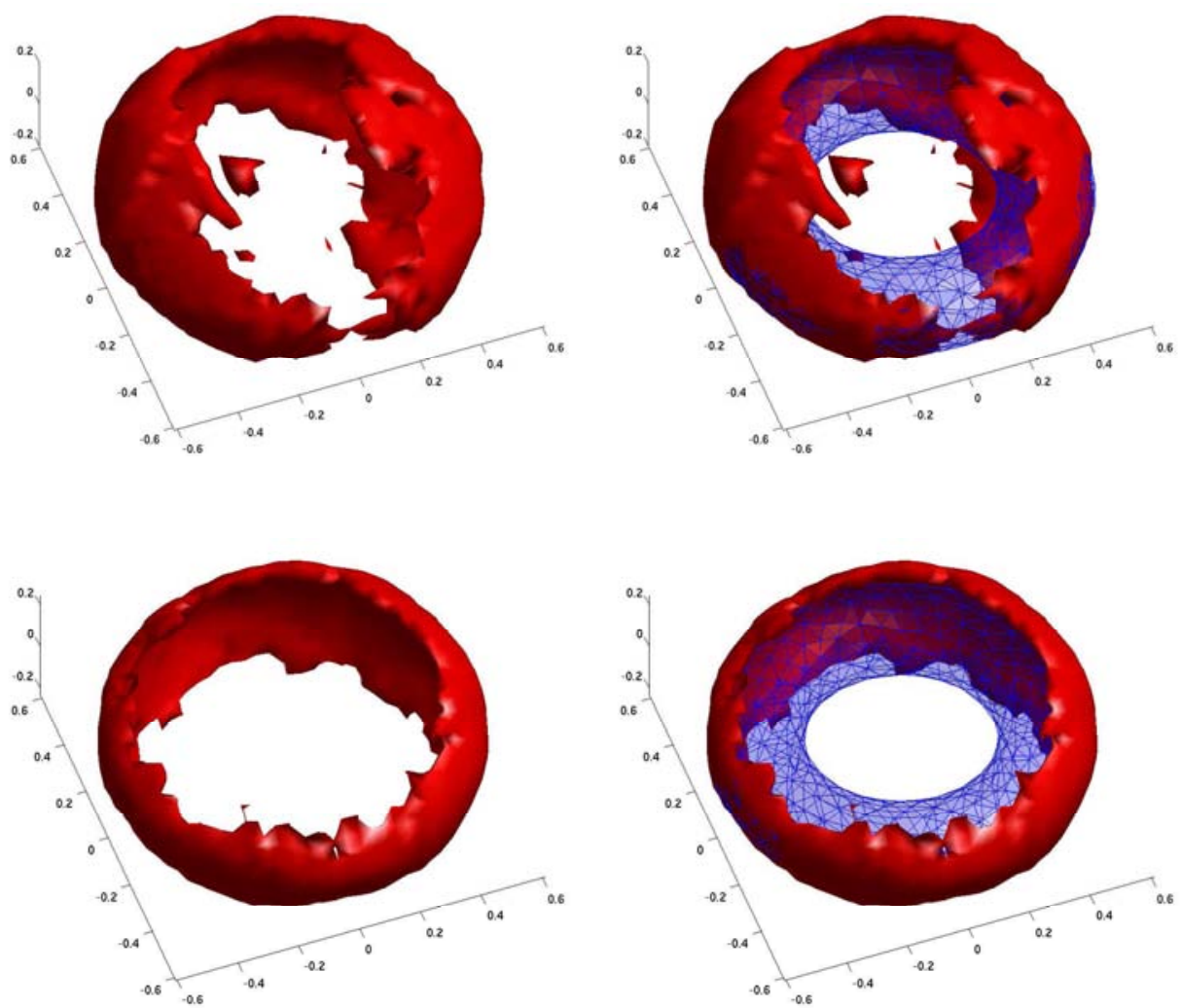

Fig. 6.27. The first row shows the isosurface of level 0.1 of the total field reconstructed with 26 configurations. The approximation domain was chosen to be a ball of radius $r=0.7$, comparable to the size of the scatterer. The second row shows the isosurface of level 0.175 of the averaged reconstructed total fields from the scattering of three different incident plane waves.

Figure 6.28 illustrates the reconstruction of the total field due to the scattering of the incident point source $u^{i}(x)=\frac{1}{4 \pi} \frac{e^{i \kappa|x|}}{|x|}$ with wave number $\kappa=2$ by the ring. For the reconstruction we used the 26 configurations (6.132) with a cut-off value $c=0.005$ for determining an approximation for the admissibility region. To reconstruct the scatterer itself we use the 26 configurations (6.131) with the smaller approximation domain given by a ball of radius 0.7 . With the same cut-off value we extracted the isosurface of level 0.00425 . If we adapt the approximation domains to the shape of the scatterer by using for example the configurations $\left(0, G_{0}^{(j)}\right)$ with the ellipsoidal approximation domains

$$
G_{0}^{(j)}=E(0.8,0.8,0.4)+S n_{j}, \quad j=1, \ldots, 26,
$$

we obtain an improved reconstruction of the ring as illustrated in Figure 6.29. Here,

$$
E(a, b, c)=\left\{(x, y, z) \in \mathbb{R}^{3}:\left(\frac{x}{a}\right)^{2}+\left(\frac{y}{b}\right)^{2}+\left(\frac{z}{c}\right)^{2} \leq 1\right\}
$$



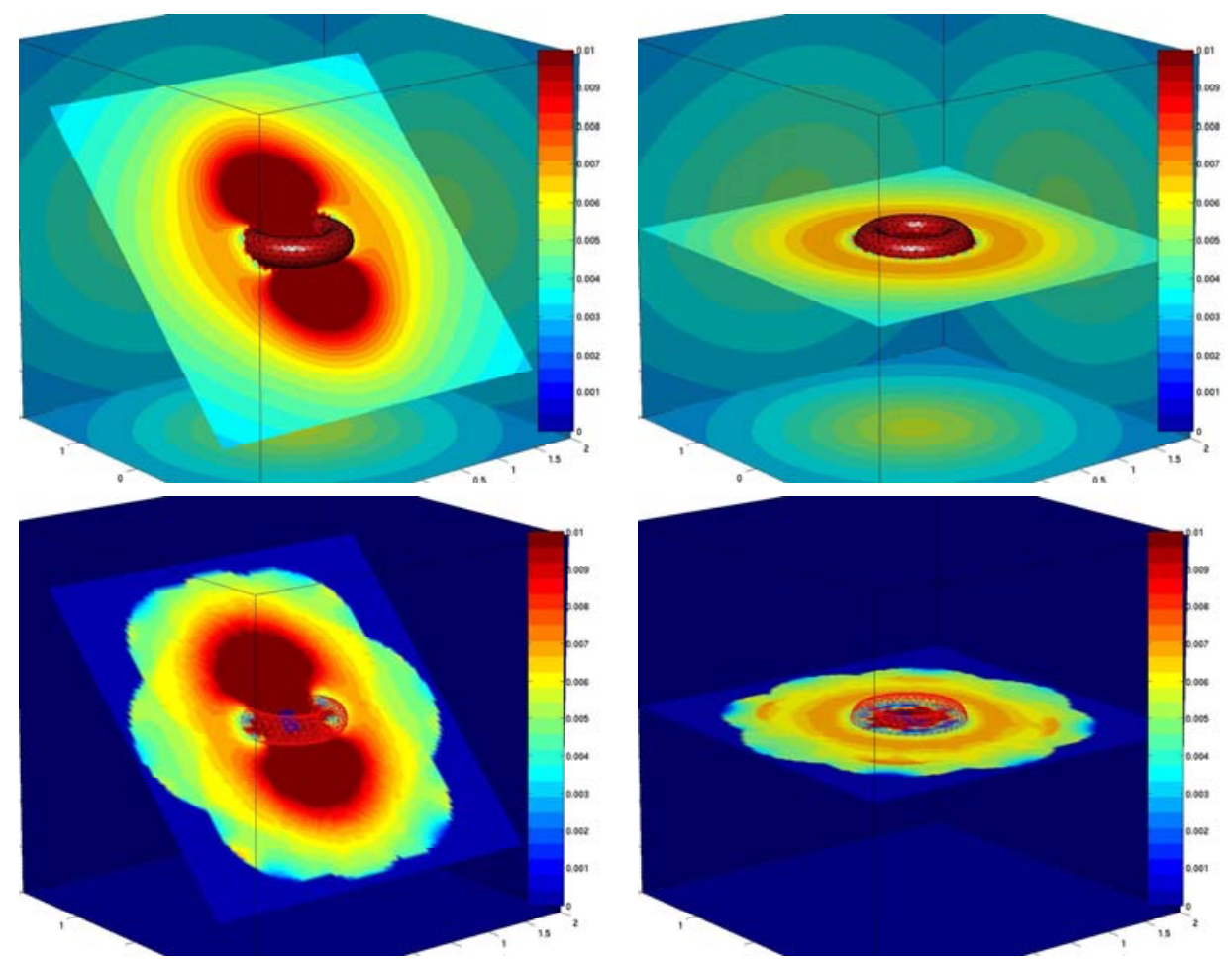

Fig. 6.28. The first row shows the modulus of the calculated total field $u$ of the direct scattering problem for the ring with an incident point source in the origin while the second row illustrates the modulus of the reconstructed total field with wave number $\kappa=2$.

denotes the ellipsoid with center in the origin and the semi-axis $a, b$ and $c$ orientated in the direction of the coordinate axis. Furthermore the operator

$$
S=1.1\left(\begin{array}{ccc}
0.8 & 0 & 0 \\
0 & 0.8 & 0 \\
0 & 0 & 0.4
\end{array}\right)
$$

is a scaling matrix that maps the direction $n_{j} \in \mathbb{S}^{2}$ to some vector $S n_{j}$ in a small distance outside the ellipsoid $E(0.8,0.8,0.4)$.

The numerical examples presented in this chapter provide a novel approach to the point source method. We have introduced a new two-step reconstruction algorithm that is based on an additional indicator function for the admissibility region. With this indicator we were able to reconstruct the scattered field in the whole admissibility region, completely. To this end we applied a suitable weighting operation in both steps of the reconstruction algorithm. Using adaptively chosen approximation domains in the second step of the algorithm we demonstrated that the PSM can identify the unknown obstacle even for nonconvex scatterers.

We explained the basic steps of this algorithm for the inverse boundary value problem for the Laplace equation in detail. Here, we reconstructed the three obstacles shown in Figure 4.1, where we used the static Herglotz 

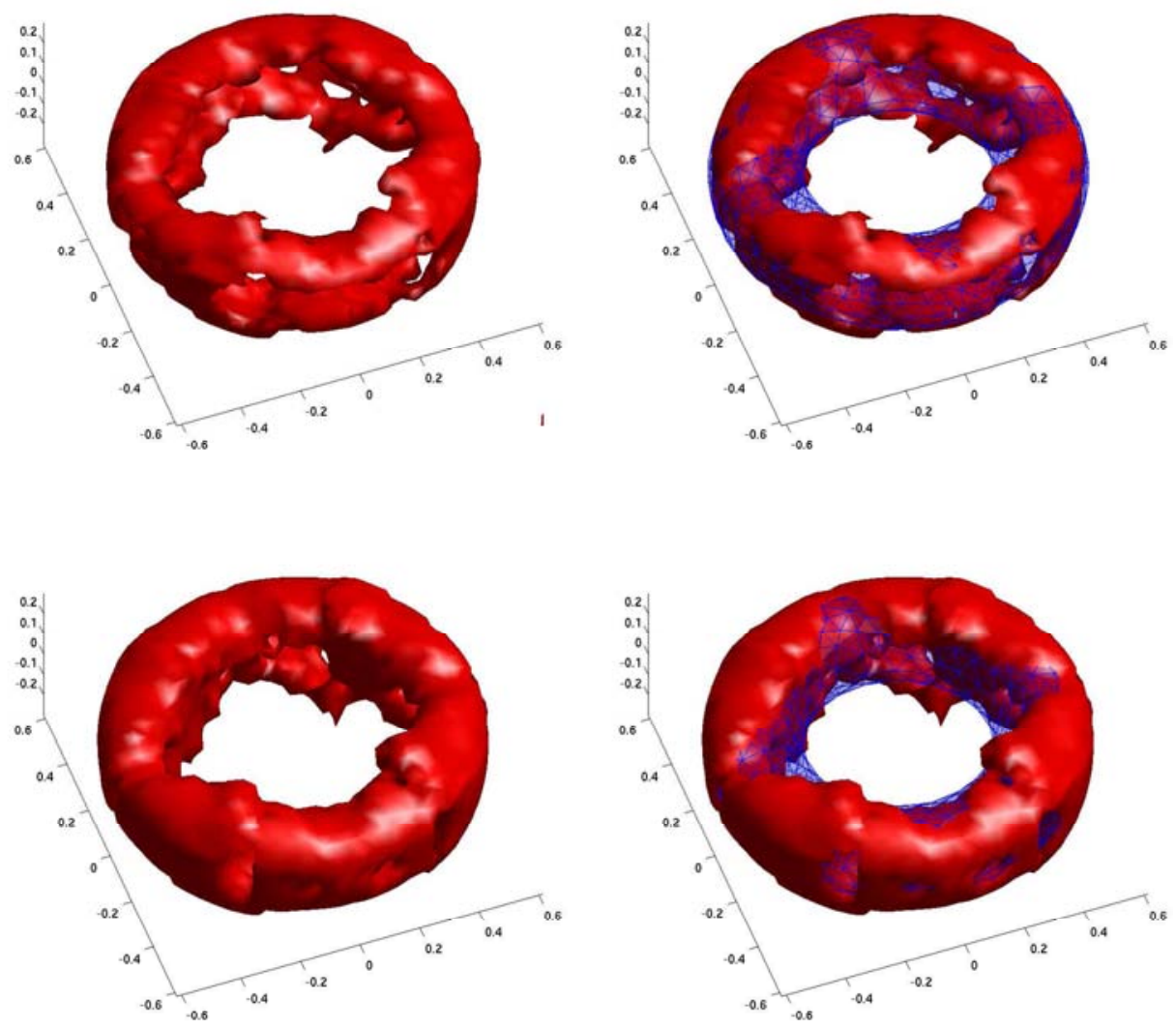

Fig. 6.29. The first row shows the isosurface of level 0.00425 of the total field due to the scattering of a point source by the ring reconstructed with 26 configurations. The approximation domain was chosen to be a ball of radius $r=0.7$. The second row shows the isosurface of level 0.0048 , where we reconstructed the field with 26 configurations and ellipsoidal approximation domains.

wave functions to speed-up the algorithm. Furthermore, we summarized the corresponding reconstructions for the inverse Helmholtz boundary value problem. These 2D reconstructions emphasize the capability of the point source method to reconstruct the complete field and to provide a full reconstruction of the unknown obstacle. Moreover, these examples demonstrate that adaptive approximation domains improve the quality of the obstacle reconstruction. In particular, we have been able to reconstruct the nonconvex kite-shaped obstacle both for the Laplace and the Helmholtz equation. Finally, we applied these new techniques to the 3D inverse scattering problem, where we reconstructed a ball and a ring-shaped obstacle. For the latter, we encountered difficulties in reconstructing the ring's hole. However, by a suitable choice of the incident field and adapted approximation domains we even obtained a rough approximation to this obstacle, too. 


\section{The Probe Method}

In the previous chapter we became acquainted with the point source method belonging to the category of decomposition methods. The basic idea of any decomposition method is to split the nonlinear ill-posed problem into a linear ill-posed problem and a non-linear but well-posed problem. While the first problem is to reconstruct the scattered field from the knowledge of the far field pattern or the Cauchy data on some measurement boundary, the second one consists in identifying the unknown obstacle from the reconstructed scattered field by the knowledge of the boundary type of the scatterer.

Since 1996 a different class of reconstruction methods has been under development which is known as sampling and probe methods. The basic idea behind these methods is to construct an indicator function which is sampled on the unknown area. Then, the shape of the obstacle can be reconstructed from the behaviour of this indicator function at the obstacle's boundary or in its interior. For a survey about sampling and probe methods we refer to [60]. Different schemes have been proposed for example by Colton and Kirsch with the linear sampling method [6], Kirsch with the factorization method [32], Potthast with the singular sources method [55], Luke and Potthast with the no response test and Kusiak, Potthast and Sylvester with the range test [63]. Masaru Ikehata contributed the enclosure method [23] and the probe method [21] to this category of reconstruction schemes.

The goal of this chapter is to present both the probe method as originally suggested by Ikehata and its numerical realization as demonstrated in [13]. For the approximation of the point source we follow the ideas of Chapter 5. In particular we develop a numerical realizable algorithm for the probe method in the same manner as demonstrated for the PSM in the previous chapter. To study the feasibility of the probe method we will use two equivalent formulations of Ikehata's indicator function and evaluate the true limiting function numerically. We will study the numerical realization of the functional proposed by Ikehata and show numerical reconstructions of sound-soft obstacles with the probe method. 


\subsection{Linear Sampling and Probe Methods}

Before we investigate the special indicator function of the probe method in detail, we provide a short survey about the main approaches and indicator functions used by different authors. Colton and Kirsch [6] use the norm $\left\|g_{z}\right\|$ of the solution to the far field equation

$$
\left(F g_{z}\right)(\hat{x})=e^{-i \kappa \hat{x} \cdot z}, \hat{x} \in \mathbb{S}^{2},
$$

for $z \in \Omega$ as indicator function, where $F$ is defined by

$$
(F g)(\hat{x})=\int_{\mathbb{S}^{2}} u^{\infty}(\hat{x}, d) g(d) d s(d)
$$

with $\hat{x} \in \mathbb{S}^{2}$. Since they solve a linear equation for each point $z$ they call this method the linear sampling method. Kirsch [32] proposed a modification of this approach using a factorization of the operator $F$. His factorization method solves the linear equation

$$
\left(\left(F^{*} F\right)^{1 / 4} g_{z}\right)(\hat{x})=e^{-i \kappa \hat{x} \cdot z}, \quad \hat{x} \in \mathbb{S}^{2},
$$

and uses the norm of $g_{z}$ as indicator function.

A different method has been proposed with the singular sources method by Potthast [55]. The idea here is to use the scattered field $\Phi^{s}(z, z)$ of incident point sources $\Phi(\cdot, z)$ as indicator function. The function can be reconstructed using the approximation techniques from Chapter 5 and it can be shown that

$$
\Phi^{s}(z, z) \rightarrow \infty, z \rightarrow \partial D,
$$

i.e. the indicator function diverges when $z$ tends to the boundary of the unknown scatterer. Recently, the singular sources method has been extended to full medium reconstructions [59] and has been used to reconstruct transmission conditions [62] as well. We will come back to the method of singular sources in the next chapter.

Further sampling and probe methods have been proposed which provide information when the far field pattern is given for one plane wave only and when the physical nature of the object is unknown. The no response test [43] constructs the response of the scatterer with respect to an incident wave which is small on some test domain. If the response is small, the unknown scatterer or a special part of its interior must be a subset of the test domain. Recent convergence results show that the no response test in its one-wave version tests for analytic continuation. If the far field pattern for many incident plane waves is given, then the no response test reconstructs the shape of the scattering object [58].

The range test by Potthast, Sylvester and Kusiak [63] uses the range of the far field pattern 


$$
\left(S^{\infty} \varphi\right)(\hat{x}):=\int_{\partial G} e^{-i \kappa \hat{x} \cdot y} \varphi(y) d s(y), \quad \hat{x} \in \mathbb{S}^{2}
$$

of the single-layer potential on the boundary of the test domain $G$. This can be employed to locate some set in the interior of the unknown scatterer without the knowledge of the boundary condition or the physical properties. If the far field is known for several incident plane waves, the full support of the scatterer can be reconstructed, see [61].

With the enclosure method Ikehata proposed a method which can reconstruct the convex hull of some scatterer from one pair of Cauchy data of the scattered wave, see [24]. He also suggested the probe method in [21], [22] and [25] for the detection of an obstacle $D$ in the inverse scattering setting. The main idea of this method is to use probes in form of point sources $\Phi(\cdot, z)$ with source point $z$ in the domain of interest to define an indicator function. Similar to the singular sources method Ikehata suggested in [21] to use an indicator function $\hat{I}(z)$ which is based on the behaviour of the integral

$$
\int_{\Omega \backslash D}\left|\nabla \Phi^{s}(\cdot, z)\right|^{2} d x
$$

and related terms. When $z$ tends to the boundary $\partial D$ of the obstacle, the integral will diverge. The indicator function can be reconstructed from Cauchy or far field data using appropriate techniques for the approximation of the point source $\Phi(\cdot, z)$ on the unknown obstacle $D$. Then, the behaviour of the indicator function can be used to locate the scatterer. Until the numerical study of the probe method presented in [13], it has not been possible to numerically implement the probe method due to the use of the nonconstructive approximation theorem of Runge in Ikehata's approach, see [25]. Meanwhile, a similar numerical realization has been published in [4] by Cheng, Liu and Nakamura. We will present the probe method in detail in the following. Moreover, we will encounter this method once more in the next chapter when investigating similarities with the singular sources method.

\subsection{The Probe Method of Ikehata}

The starting points of Ikehata's probe method are the Dirichlet to Neumann (D-t-N) maps $\Lambda$ for the direct obstacle boundary value problem and $\Lambda_{0}$ for the obstacle-free boundary value problem. The D-t-N map sets up a correspondence between the boundary values of a solution to some boundary value problem and the normal derivative of the solution at the boundary. In practice, the D-t- $\mathrm{N}$ map is often available via physical measurements. For our numerical study we simulate the D-t-N maps by solving their underlying boundary value problems explicitly. Therefore, we will recall the direct obstacle boundary value problem and the corresponding obstacle-free 
boundary value problem first. Note, that we use the notation $\Omega_{D}:=\Omega \backslash \bar{D}$ in the following.

Direct obstacle boundary value problem. For a given differentiable function $f \in C^{1, \alpha}(\partial \Omega), 0<\alpha<1$ with Hölder continuous derivative and a continuous function $g \in C(\partial D)$, we want to find a function $u \in C^{2}\left(\Omega_{D}\right) \cap$ $C\left(\overline{\Omega_{D}}\right)$ such that

$$
\begin{aligned}
\Delta u+\kappa^{2} u=0 & \text { in } \Omega_{D}, \\
u=f & \text { on } \partial \Omega, \\
u=g & \text { on } \partial D .
\end{aligned}
$$

In contrast to the definition (4.35) - (4.36) of the direct boundary value problem we additionally assume Hölder continuity of the derivative of the boundary data on the measurement boundary $\partial \Omega$. This ensures the existence of the normal derivative of the solution on $\partial \Omega$.

Obstacle-free boundary value problem. For a given continuous function $f \in C(\partial \Omega)$ we want to find a function $v \in C^{2}(\Omega) \cap C(\bar{\Omega})$ such that

$$
\begin{aligned}
\Delta v+\kappa^{2} v & =0 & & \text { in } \Omega, \\
v & =f & & \text { on } \partial \Omega .
\end{aligned}
$$

We tacitly assume in the following, that $-\kappa^{2}$ is not an eigenvalue of the Laplacian in $\Omega_{D}$. Then, by a straightforward modification of Theorem 4.8, the direct obstacle boundary value problem has a unique solution, see [13]. An analogous result holds for the obstacle-free boundary value problem, but since we do not need its numerical solution for our study of the probe method we will omit it here.

Next, we consider the Dirichlet to Neumann maps $\Lambda$ and $\Lambda_{0}$ of the direct obstacle and obstacle-free boundary value problem.

Dirichlet to Neumann map $\Lambda$. The D-t-N map $\Lambda$ of the direct obstacle boundary value problem maps a Hölder continuously differentiable function $f \in C^{1, \alpha}(\partial \Omega)$ to the continuous function $h \in C^{0, \alpha}(\partial \Omega)$ defined by

$$
h(x):=\frac{\partial u}{\partial \nu}(x)
$$

for $x \in \partial \Omega$, where $u$ solves the direct obstacle boundary value problem $(7.7)$ - (7.9) with a sound-soft boundary condition on $\partial D$, i.e. $u$ equals $g \equiv 0$ on $\partial D$.

Dirichlet to Neumann map $\Lambda_{0}$. The D-t-N map $\Lambda_{0}$ of the obstaclefree boundary value problem maps a continuous function $f \in C(\partial \Omega)$ to the continuous function $h_{0} \in C(\partial \Omega)$ given by

$$
h_{0}(x):=\frac{\partial v}{\partial \nu}(x)
$$


for $x \in \partial \Omega$, where $v$ solves the obstacle-free boundary value problem (7.10) (7.11).

As mentioned before, we simulate the $\mathrm{D}$-t- $\mathrm{N}$ map $\Lambda$ in our numerical studies of the probe method. We realize this in the following way. For a given Hölder continuously differentiable function $f$ we solve the direct obstacle boundary value problem (7.7) - (7.9) with a sound-soft boundary condition $g \equiv 0$ on $\partial D$ using the combined layer ansatz

$$
\begin{aligned}
u(x) & =\int_{\partial D}\left\{\frac{\partial \Phi(x, y)}{\partial \nu(y)}-i \eta \Phi(x, y)\right\} \varphi_{1}(y) d s(y) \\
& +\int_{\partial \Omega} \frac{\partial \Phi(x, y)}{\partial \nu(y)} \varphi_{2}(y) d s(y), \quad x \in \Omega_{D},
\end{aligned}
$$

with a positive number $\eta$ as demonstrated in Chapter 4. Here we assume continuity of the density $\varphi_{1} \in C(\partial D)$ and Hölder continuity of the density $\varphi_{2} \in C^{1, \alpha}(\partial \Omega)$ with $0<\alpha<1$.

Now, we calculate the normal derivative of $u$ at the boundary $\partial \Omega$ numerically in order to obtain $h=\Lambda f$. However, the behaviour of the combined layer potential (7.14) at the boundary $\partial \Omega$ forbids to apply a simple difference quotient for calculating $h$. Instead, we differentiate the combined layer potential analytically and use the notation (3.33) with $\Gamma_{1}=\partial D$ and $\Gamma_{2}=\partial \Omega$ to get the expression

$$
h(x)=\frac{1}{2}\left(\left(T_{21} \varphi_{1}\right)(x)-i \eta\left(K_{21}^{\prime} \varphi_{1}\right)(x)+\left(T_{22} \varphi_{2}\right)(x)\right), \quad x \in \partial \Omega,
$$

for the normal derivative on the boundary $\partial \Omega$. Here, $T_{2 k}$ denotes the integral operator

$$
\left(T_{2 k} \varphi_{2}\right)(x):=2 \frac{\partial}{\partial \nu(x)} \int_{\Gamma_{k}} \frac{\partial \Phi(x, y)}{\partial \nu(y)} \varphi_{k}(y) d s(y), \quad x \in \partial \Omega,
$$

which is hypersingular for $k=2$. Furthermore, $K_{21}^{\prime}$ is the adjoint operator (3.31) of the double-layer operator $K_{21}$. Since the kernels of the integral operators $T_{21}: C(\partial D) \rightarrow C(\partial \Omega)$ and $K_{21}^{\prime}: C(\partial D) \rightarrow C(\partial \Omega)$ are analytic both operators can easily be evaluated analytically or with a simple numerical differentiation scheme.

For the numerical evaluation of the hypersingular integral operator $T_{22}$ we apply Maue's identity

$$
T_{22} \varphi_{2}=\frac{d}{d s} S_{22} \frac{d \varphi_{2}}{d s}+\kappa^{2} \nu \cdot S_{22}\left(\nu \varphi_{2}\right)
$$

see [44]. After parametrizing (7.17) and integrating by parts we obtain

$$
\begin{aligned}
& \left(\frac{d}{d s} S_{22} \frac{d \varphi_{2}}{d s}\right)(x(t)) \\
& =\frac{1}{\left|x^{\prime}(t)\right|} \int_{0}^{2 \pi}\left\{\frac{1}{2 \pi} \cot \frac{\tau-t}{2} \frac{d \varphi_{2}(x(\tau))}{d \tau}-N(t, \tau) \varphi_{2}(x(\tau))\right\} d \tau(7 .
\end{aligned}
$$


with

$$
N(t, \tau)=N_{1}(t, \tau) \ln \left(4 \sin ^{2} \frac{t-\tau}{2}\right)+N_{2}(t, \tau)
$$

and analytic functions $N_{1}(t, \tau)$ and $N_{2}(t, \tau)$. For the integration of these terms we use the interpolatory quadrature rules

$$
\frac{1}{2 \pi} \int_{0}^{2 \pi} \cot \frac{\tau-t}{2} f^{\prime}(\tau) d \tau \approx \sum_{j=0}^{2 n-1} T_{j}^{(n)}(t) f\left(t_{j}^{(n)}\right)
$$

and

$$
\int_{0}^{2 \pi} \ln \left(4 \sin ^{2} \frac{t-\tau}{2}\right) f(\tau) \approx \sum_{j=0}^{2 n-1} R_{j}^{(n)}(t) f\left(t_{j}^{(n)}\right)
$$

with the weights

$$
T_{j}^{(n)}(t)=-\frac{1}{n} \sum_{m=1}^{n-1} m \cos m\left(t-t_{j}^{(n)}\right)-\frac{1}{2} \cos n\left(t-t_{j}^{(n)}\right)
$$

and

$$
R_{j}^{(n)}(t)=-\frac{2 \pi}{n} \sum_{m=1}^{n-1} \frac{1}{m} \cos m\left(t-t_{j}^{(n)}\right)-\frac{\pi}{n^{2}} \cos n\left(t-t_{j}^{(n)}\right) .
$$

Here, $t_{j}^{(n)}=\frac{j \pi}{n}, j=0, \ldots, 2 n-1$ are $2 n$ equidistant sampling points of the interval $[0,2 \pi)$. Furthermore we apply the trapezoidal rule for the integration of the kernel $N_{2}$. For a detailed study of this operator and explicit expressions for the kernels $N_{1}$ and $N_{2}$ we refer to the work [35] of Kress.

Here, we just motivate formula $(7.18)$ by looking at the static case, i.e. we consider the hypersingular integral operator $T_{22}$ with the fundamental solution

$$
\Phi(x, y)=\frac{1}{2 \pi} \log \frac{1}{|x-y|}
$$

of Laplace's equation. Using Maue's identity

$$
T_{22} \varphi_{2}=\frac{d}{d s} S_{\Omega} \frac{d \varphi_{2}}{d s}
$$

in the static case, see for example Theorem 7.28 in [36], and inserting the parametrization $x(t)$ of the boundary $\partial \Omega$ we derive

$$
2 \frac{1}{\left|x^{\prime}(t)\right|} \int_{0}^{2 \pi} \frac{\partial}{\partial t} \frac{1}{2 \pi} \log \frac{1}{|x(t)-x(\tau)|} \frac{d \varphi_{2}(x(\tau))}{d \tau} d \tau, \quad 0 \leq t \leq 2 \pi .
$$

With the splitting (4.10) of the single-layer potential and an integration by parts we obtain 


$$
\begin{aligned}
& \left(T_{22} \varphi_{2}\right)(x(t))= \\
& =-\frac{1}{\left|x^{\prime}(t)\right|} \int_{0}^{2 \pi} \frac{\partial}{\partial t} \frac{1}{2 \pi}\left(\log 4 \sin ^{2} \frac{t-\tau}{2}+\rho(t, \tau)\right) \frac{d \varphi_{2}(x(\tau))}{d \tau} d \tau \\
& =\frac{1}{\left|x^{\prime}(t)\right|} \int_{0}^{2 \pi}\left(\frac{1}{2 \pi} \cot \frac{\tau-t}{2} \frac{d \varphi_{2}(x(\tau))}{d \tau}+\frac{1}{2 \pi} N(t, \tau) \varphi_{2}(x(\tau))\right) d \tau
\end{aligned}
$$

where the kernel $N(t, \tau)$ is given by

$$
N(t, \tau)=\frac{\partial^{2}}{\partial \tau \partial t} \rho(t, \tau)=\frac{\partial^{2}}{\partial \tau \partial t} \log \frac{|x(t)-x(\tau)|^{2}}{4 \sin ^{2} \frac{t-\tau}{2}}, \quad t \neq \tau .
$$

After calculating these derivatives a Taylor expansion and a lot of patience shows that the kernel $N(t, \tau)$ is continuous and can be represented in the form

$$
\begin{aligned}
N(t, \tau)= & -2 \frac{x^{\prime}(t) \cdot x^{\prime}(\tau)}{|x(t)-x(\tau)|^{2}}-\frac{1}{2 \sin ^{2} \frac{t-\tau}{2}} \\
& +4 \frac{(x(t)-x(\tau)) \cdot x^{\prime}(\tau)(x(t)-x(\tau)) \cdot x^{\prime}(t)}{|x(t)-x(\tau)|^{4}}, \quad t \neq \tau,
\end{aligned}
$$

with the diagonal term

$$
N(t, t)=-\frac{1}{6}+\frac{1}{2} \frac{\left|x^{\prime \prime}(t)\right|^{2}}{\left|x^{\prime}(t)\right|^{2}}-\frac{\left(x^{\prime}(t) \cdot x^{\prime \prime}(t)\right)^{2}}{\left|x^{\prime}(t)\right|^{4}}+\frac{1}{3} \frac{x^{\prime} \cdot x^{\prime \prime \prime}}{\left|x^{\prime}\right|^{2}} .
$$

With the hypersingular integral operator $T_{22}$ we are able to simulate the $\mathrm{D}-\mathrm{t}-\mathrm{N}$ map $\Lambda$ of the obstacle boundary value problem by solving the direct boundary value problem with given boundary data $f \in C^{1, \alpha}(\partial \Omega)$ with the combined layer ansatz (7.14) first. To this end we apply Nyström's method to obtain an approximate solution of the boundary integral equation (4.41). Then we insert the approximating density into the discretized form of integral equation (7.15) using the quadrature formulae (7.20), (7.21) together with the trapezoidal rule for the integration of the hypersingular part, the part with the logarithmic singularity and the analytic part of the occurring integral operators, respectively. Since this procedure demands only slight modifications to the numerical implementation of the forward solver presented in Chapter 4, we skip the details and return to the indicator function of the probe method.

For the reconstruction of the obstacle $D$ the probe method uses the indicator functional

$$
I(f):=\int_{\partial \Omega} \overline{\left(\Lambda-\Lambda_{0}\right) f(y)} \cdot f(y) d s(y),
$$

with $f \in C^{1, \alpha}(\partial \Omega)$, and given $\mathrm{D}-\mathrm{t}-\mathrm{N}$ maps $\Lambda$ and $\Lambda_{0}$. For any $z \in \Omega$ we choose a domain of approximation $G(z)$ with $z \notin G(z)$ and find a solution 
$v_{z, \varepsilon} \in C^{2}(\Omega) \cap C^{1, \alpha}(\bar{\Omega})$ to the Helmholtz equation in $\Omega$, which approximates the point source $\Phi(\cdot, z)$ on the approximation domain $G(z)$ as demonstrated in Chapter 5. In particular, for every $\varepsilon>0$ we can find a Herglotz wave function $v_{z, \varepsilon}$ such that

$$
\left\|\Phi(\cdot, z)-v_{z, \varepsilon}\right\|_{L^{2}(G(z))} \leq \varepsilon
$$

We use the notation

$$
\hat{I}(z):=\lim _{\varepsilon \rightarrow 0} I\left(f_{z, \varepsilon}\right),
$$

where $f_{z, \varepsilon}:=\left.v_{z, \varepsilon}\right|_{\partial \Omega}$ and $I$ is defined in (7.31). In the admissibility region $\mathcal{A}=\{z \in \Omega: \bar{D} \subset G(z)\}$ the function $\hat{I}$ is well defined in view of the following theorem.

Theorem 7.1. If for some $z \in \Omega$ we have $\bar{D} \subset G(z), z \notin G(z)$, then the limit $\hat{I}(z)$ exists. Let $z_{j}$ be a sequence of points such that $\bar{D} \subset G\left(z_{j}\right), z_{j} \notin$ $G\left(z_{j}\right)$ for $j \in \mathbb{N}$ and $z_{j} \rightarrow \partial D$ for $j \rightarrow \infty$. Then, we have

$$
\left|\operatorname{Re} \hat{I}\left(z_{j}\right)\right| \rightarrow \infty, \quad j \rightarrow \infty .
$$

The proof can easily be adapted from Theorem 3.1 in [5].

In the paper [5] which is based upon the work of Ikehata, see for example [21], [22] and [25], a needle approach is proposed to reconstruct the obstacle $D$. The authors define a needle as a continuous function $c:[0,1] \longrightarrow \bar{\Omega}$ with $c(0), c(1) \in \partial \Omega$ and $c(t) \in \Omega$ for $0<t<1$ and evaluate the function $\hat{I}$ for the points $z=c(t)$ on the needle. In this work they choose the domains

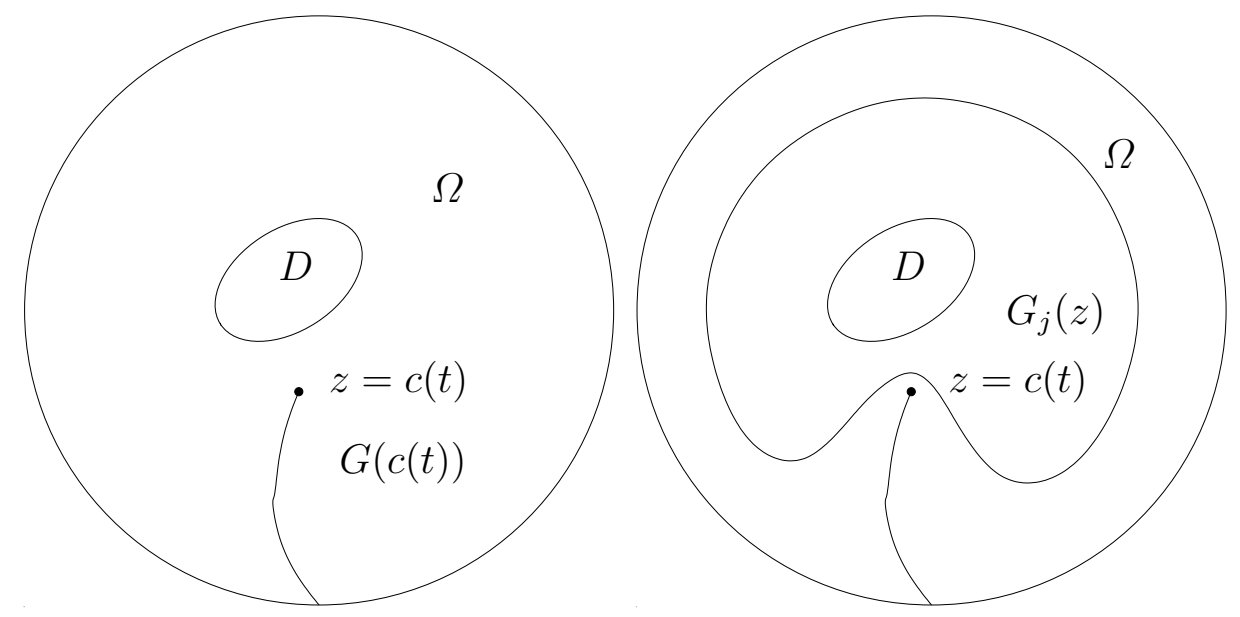

Fig. 7.1. The needle domain $G(c(t))$ (left) and an approximation $G_{j}(z)$ (right) to the needle domain $G(c(t))$.

$$
G(z)=G(c(t))=\Omega \backslash\{c(\tau): 0<\tau \leq t\}
$$


for the approximation of the point source $\Phi(\cdot, c(t))$ in a Sobolev space setting under the constraint that the support of the approximating function restricted to the boundary $\partial \Omega$ is contained in a prescribed open set $\Gamma \subset \partial \Omega$. They prove the existence of such an approximating function with Runge's approximation theorem, which uses the Hahn-Banach theorem and leads therefore to a nonconstructive approximation method. Figure 7.1 illustrates this special choice of approximation domains.

The approximation domains (7.35) are very suitable for theoretical reasons, since the boundary can then be described easily by the set

$$
\partial D=\{c(t): t=T(c), c \text { is a needle with } T(c)<1\},
$$

with the impact parameter

$$
T(c)=\sup \left\{0<t<1: \sup _{0 \leq s \leq t}|\operatorname{Re} \hat{I}(c(s))|<\infty\right\} .
$$

In principle, the approximation techniques of Chapter 5 can be used to construct the approximations of the point source needed for the probe method. This can be carried out by using a family of approximation domains $G_{j}(z)$, $j=1,2,3, \ldots$, which step by step fill the whole needle domain $G(z)$ as defined in (7.35). Then, for every compact subset $M$ of $G(z)$ we can find an index $j \in \mathbb{N}$ such that $M \subset G_{j}(z)$ and on $M$ we obtain an approximation of $\Phi(\cdot, z)$ with an Herglotz wave function

$$
v_{z, \varepsilon}(x)=\int_{\mathbb{S}^{m-1}} e^{i \kappa x \cdot d} g_{z, \varepsilon}(d) d s(d), \quad x \in M
$$

from the solution $g_{z, \varepsilon}$ to the regularized integral equation

$$
\left(\alpha I+H^{*} H\right) g_{z, \varepsilon}=\left.H^{*} \Phi(\cdot, z)\right|_{\partial G_{j}(z)} .
$$

However, this process is numerically instable. When the non-convexity of $G_{j}$ becomes larger, then the norm of the density of the approximating Herglotz wave function soon explodes and numerical approximations become almost impossible. To emphasize this point we refer to the numerical study [4] of Nakamura et al. which is based on this idea of exhausting the needle domains.

For this reason, we restrict our numerics to some simple approximation domains $G_{j}(z)$ and do not carry out the limit $j \rightarrow \infty$ to exhaust the needle domains as illustrated in Figure 7.1. In our numerical implementation of the probe method we will again start with circular approximation domains which are then adjusted during the reconstruction procedure as demonstrated in the previous chapter. Since we do not have the characterization (7.36) in this case we additionally calculate an approximation $\mathcal{E}$ of the admissibility region $\mathcal{A}$, where the evaluation of the indicator function $\hat{I}$ is justified. 


\subsection{Numerical Implementation of the Probe Method}

For the numerical implementation of the probe method we may again choose a fixed reference configuration $\left(0, G_{0}\right)$ and use translations and rotations of this reference configuration for the choice of the approximation domains. In this case, we can also make use of Theorem 5.12 to obtain the following efficient algorithm for the probe method.

Definition 7.2 (Probe method for BVPs). For the boundary value problem (6.1) - (6.3) the probe method calculates an indicator function I for the obstacle $D$ on subsets $\mathcal{E}_{c}$ of $\mathcal{B}$ by the following steps:

1. Choose a fixed reference configuration $\left(0, G_{0}\right)$ such that $0 \notin G_{0}$ and calculate the density $g_{0, \varepsilon} \in L^{2}\left(\mathbb{S}^{m-1}\right)$ as a solution of equation (5.89) with $H$ defined by (5.45).

2. For each $z \in \mathcal{S} \subset \mathcal{B}$ choose a domain of approximation $G(z)$ of the form (5.86), i.e. the configuration $(z, G(z))$ is an orthogonal translation of the reference configuration $\left(0, G_{0}\right)$.

3. For each $z \in \mathcal{S}$ calculate the density $g_{z, \varepsilon} \in L^{2}\left(\mathbb{S}^{m-1}\right)$ via (5.91).

4. Calculate $f_{z, \varepsilon}:=\left.v_{z, \varepsilon}\right|_{\partial \Omega}$ and $\Lambda_{0} f_{z, \varepsilon}$ for all $z \in \mathcal{S}$ with (7.38).

5. a) Solve the direct obstacle boundary value problem (7.7) - (7.9) with $f=f_{z, \varepsilon}$ and $g=0$ for all $z \in \mathcal{S}$. Then, calculate $\Lambda f_{z, \varepsilon}$ with equation (7.15), (simulation of the $D-t-N$ map).

or

b) Calculate $\Lambda f_{z, \varepsilon}$ by some externally given $D-t-N$ map for all $z \in \mathcal{S}$.

6. Calculate the approximation $I\left(f_{z, \varepsilon}\right) \approx \hat{I}(z)$ via (7.31) for all $z \in \mathcal{S}$.

7. Calculate $\left|\operatorname{Re}\left(I\left(f_{z, \varepsilon}\right)\right)\right|$ for all $z \in \mathcal{S}$.

8. Repeat steps $3-7$ with a different error level $\varepsilon$ and calculate an approximation $\mathcal{E}_{c}:=\left\{z \in \mathcal{S}:\left|\mathcal{I}_{\text {enl }}(z)\right|<c\right\}$ for the admissibility region.

9. Repeat steps $1-8$ with a different choice of the approximation domains until the union of the domains $\mathcal{E}_{c}$ is a doubly connected domain. Extend the single indicator functions I with a suitable weighted mean operation to this domain.

10. Choose some suitable cut-off constant $C>0$ and decide

a) $z \in D$ if $\left|\operatorname{Re}\left(I\left(f_{z, \varepsilon}\right)\right)\right|>C$,

b) $z \in \mathbb{R}^{m} \backslash \bar{D}$ if $\left|\operatorname{Re}\left(I\left(f_{z, \varepsilon}\right)\right)\right| \leq C$,

for all $z \in \mathcal{E}_{c}$.

We remark here, that the decision criterion in step 10 is justified only in the case, when $D \subset G(z)$. In contrast to the limiting case of the needle approach, where $D \subset G(c(t))$ is always satisfied as long as $t<T(c)$, we do not have information about whether $D \subset G(z)$ or not. Therefore we first calculate an approximation for the admissibility region in step 8 in analogy to the algorithm of the PSM. For the probe method we test the condition $D \subset G(z)$ with the indicator function 


$$
\mathcal{I}_{\text {enl }}(z):=I\left(f_{z, \varepsilon_{1}}\right)-I\left(f_{z, \varepsilon_{2}}\right), \quad z \in \mathcal{S}
$$

of the admissibility region. Then, we repeat this procedure in step 9 until the union of the regions $\mathcal{E}_{c}$ enlightens a whole region around the scatterer. Furthermore, the following lemma provides an equivalent formulation of the Ikehata functional (7.31) without using the D-t-N maps.

Recall that $u$ denotes the solution to the direct obstacle boundary value problem (7.7) - (7.9) with boundary values $f$ on $\partial \Omega$ and $g \equiv 0$ on $\partial D$. Furthermore $v$ denotes the solution to the obstacle-free boundary value problem $(7.10)$ - (7.11) with boundary data $f$ on $\partial \Omega$. Then, we have the following reformulation for the Ikehata functional for a sound-soft obstacle, which can be deduced from a more general result in [5].

Lemma 7.3. For a real wave number $\kappa \in \mathbb{R}$ and a sound-soft obstacle $D$ the Ikehata functional is given by

$$
I(f)=\int_{\Omega_{D}}\left(|\nabla w|^{2}-\kappa^{2}|w|^{2}\right) d x+\int_{D}\left(|\nabla v|^{2}-\kappa^{2}|v|^{2}\right) d x
$$

with $w:=u-v$.

Proof. From Green's formula together with $u=0$ on $\partial D$ we obtain

$$
\begin{aligned}
\int_{\Omega_{D}}|\nabla(u-v)|^{2} d x= & \int_{\partial \Omega} u \frac{\partial(\bar{u}-\bar{v})}{\partial \nu} d s+\kappa^{2} \int_{\Omega_{D}} u(\bar{u}-\bar{v}) d x \\
& -\int_{\Omega_{D}} \nabla v \nabla(\bar{u}-\bar{v}) d x \\
= & \left\langle\left(\Lambda_{D}-\Lambda_{0}\right) \bar{f}, f\right\rangle+\kappa^{2} \int_{\Omega_{D}} u(\bar{u}-\bar{v}) d x \\
& -\int_{\Omega_{D}} \nabla v \nabla \bar{u} d x+\int_{\Omega_{D}}|\nabla v|^{2} d x .
\end{aligned}
$$

Using $\nabla v \cdot \nabla \bar{u}=\nabla \cdot(\bar{u} \nabla v)-\bar{u} \Delta v$ and the divergence theorem of Gauss we get

$$
\begin{aligned}
-\int_{\Omega_{D}} \nabla v \nabla \bar{u} d x+\int_{\Omega_{D}}|\nabla v|^{2} d x= & -\int_{\partial \Omega} \bar{u} \frac{\partial v}{\partial \nu} d s-\kappa^{2} \int_{\Omega_{D}} \bar{u} v d x \\
& +\int_{\Omega}|\nabla v|^{2} d x-\int_{D}|\nabla v|^{2} d x,
\end{aligned}
$$

and again with $\nabla v \cdot \nabla \bar{v}=\nabla \cdot(\bar{v} \nabla v)-\bar{v} \Delta v$ we observe

$$
\begin{aligned}
-\int_{\Omega_{D}} \nabla v \nabla \bar{u} d x+\int_{\Omega_{D}}|\nabla v|^{2} d x= & -\kappa^{2} \int_{\Omega_{D}} \bar{u} v d x+\kappa^{2} \int_{\Omega}|v|^{2} d x \\
& -\int_{D}|\nabla v|^{2} d x
\end{aligned}
$$


since $u-v=0$ on $\partial \Omega$. With this expression we can reformulate equation (7.42) to

$$
\begin{aligned}
I(f)= & \left\langle\left(\Lambda_{D}-\Lambda_{0}\right) \bar{f}, f\right\rangle \\
= & \int_{\Omega_{D}}\left(|\nabla w|^{2}-\kappa^{2} u(\bar{u}-\bar{v})+\kappa^{2} \bar{u} v\right) d x \\
& -\kappa^{2} \int_{\Omega}|v|^{2} d x+\int_{D}|\nabla v|^{2} d x \\
= & \int_{\Omega_{D}}\left(|\nabla w|^{2}-\kappa^{2}(u-v)(\bar{u}-\bar{v})\right) d x+\kappa^{2} \int_{\Omega_{D}}|v|^{2} d x \\
& -\kappa^{2} \int_{\Omega}|v|^{2} d x+\int_{D}|\nabla v|^{2} d x \\
= & \int_{\Omega_{D}}\left(|\nabla w|^{2}-\kappa^{2}|w|^{2}\right) d x+\int_{D}\left(|\nabla v|^{2}-\kappa^{2}|v|^{2}\right) d x
\end{aligned}
$$

which ends the proof.

If we insert the boundary values $f_{z, \varepsilon}$ of the approximating Herglotz wave function $v_{z, \varepsilon}$ on the boundary $\partial \Omega$ into (7.41) we obtain

$$
I\left(f_{z, \varepsilon}\right)=\int_{\Omega_{D}}\left(\left|\nabla w_{z, \varepsilon}\right|^{2}-\kappa^{2}\left|w_{z, \varepsilon}\right|^{2}\right) d x+\int_{D}\left(\left|\nabla v_{z, \varepsilon}\right|^{2}-\kappa^{2}\left|v_{z, \varepsilon}\right|^{2}\right) d x
$$

Here $w_{z, \varepsilon}:=u_{z, \varepsilon}-v_{z, \varepsilon}$ where $u_{z, \varepsilon}$ denotes the solution to the boundary value problem $(7.7)-(7.9)$ with the boundary values $f$ replaced by $f_{z, \varepsilon}$. Note that, by construction, $v_{z, \varepsilon}$ is a solution to the boundary value problem $(7.10)$ - (7.11) with the boundary values $f_{z, \varepsilon}$. For an admissible approximation domain $G(z)$ the function $v_{z, \varepsilon}$ approximates the fundamental solution on $D$ in view of $\bar{D} \subset G(z)$ and Theorem 5.7. Hence, due to the well-posedness of the direct problems $(7.7)-(7.9)$ and $(7.10)-(7.11)$ we can make the difference $\mathcal{I}_{\text {enl }}(z)=I\left(f_{z, \varepsilon_{1}}\right)-I\left(f_{z, \varepsilon_{2}}\right)$ arbitrarily small. To this end we choose two different error levels $\varepsilon_{1}$ and $\varepsilon_{2}$ close to each other and compute the corresponding densities $g_{z, \varepsilon_{i}}$ of the Herglotz wave functions $v_{z, \varepsilon_{i}}$ for $i=1,2$ with a Tikhonov regularization scheme. In contrary, if $G(z)$ is not admissible, the oscillating behaviour of $v_{z, \varepsilon}$ outside its approximation domain will affect both boundary values for $w_{z, \varepsilon}$ and $v_{z, \varepsilon}$ on $\partial D \not \subset G(z)$ and thus the indicator function $\mathcal{I}_{\text {enl }}$ itself. Hence we expect $\mathcal{I}_{\text {enl }}(z)$ to provide a good approximation $\mathcal{E}_{c}=\left\{z \in \mathcal{S}:\left|\mathcal{I}_{\text {enl }}(z)\right|<c\right\}$ for the admissibility region $\mathcal{A}$, where $c>0$ denotes the cut-off value of the indicator function $\mathcal{I}_{\text {enl }}$.

Since the domain of integration is unknown for both integrals in (7.43), this formulation of the Ikehata functional cannot be used for reconstructions. Nevertheless, it will serve for a comparison with the D-t-N formulation (7.31) of the Ikehata functional in two ways. First, with the help of Lemma 7.3 we can calculate the Ikehata functional for all $z \in \Omega_{D}$ without 
any approximation of the point source $\Phi(\cdot, z)$. To this end, we replace $v_{z, \varepsilon}$ and $w_{z, \varepsilon}$ in equation (7.43) by $v_{z, 0}=\Phi(\cdot, z)$ and $w_{z, 0}$, which denotes the solution to the boundary value problem

$$
\begin{aligned}
\Delta w+\kappa^{2} w & =0 \quad \text { in } \Omega_{D}, \\
w & =0 \quad \text { on } \partial \Omega, \\
w & =-\Phi(\cdot, z) \quad \text { on } \partial D .
\end{aligned}
$$

Secondly, we can also evaluate the Ikehata functional (7.41) with approximate functions $v_{z, \varepsilon}$ and $w_{z, \varepsilon}$ to illustrate the influence of the approximation of the point source function on the Ikehata functional. Here, $v_{z, \varepsilon} \approx \Phi(\cdot, z)$ is defined by equation (7.38) and (7.39), and $w_{z, \varepsilon}$ is the solution to the boundary value problem

$$
\begin{aligned}
\Delta w+\kappa^{2} w & =0 \quad \text { in } \Omega_{D}, \\
w & =0 \quad \text { on } \partial \Omega, \\
w & =-\left.v_{z, \varepsilon}\right|_{\partial D} \quad \text { on } \partial D .
\end{aligned}
$$

Both domain integrals in (7.43) can numerically be evaluated with the rectangle rule. The second integral can be easily calculated for any point $z \in \Omega_{D}$ with both the approximate function $v_{z, \varepsilon}$ (7.38) and the point source function $v_{z, 0}=\Phi(\cdot, z)$ itself. The gradients of these functions are given by

$$
\nabla v_{z, \varepsilon}(x)=i \kappa \int_{\mathbb{S}} d e^{i \kappa x \cdot d} g_{z, \varepsilon}(d) d s(d)
$$

and

$$
\nabla v_{z, 0}(x)=-\frac{i \kappa}{4} H_{1}^{(1)}(\kappa|x-z|) \frac{x-z}{|x-z|} .
$$

For the first integral, we have to solve the direct obstacle boundary value problem $(7.7)-(7.9)$ with boundary values $f=0, g=-\left.v_{z, \varepsilon}\right|_{\partial D}$ to obtain $w_{z, \varepsilon}$ and with boundary data $f=0, g=-\left.\Phi(\cdot, z)\right|_{\partial D}$ to obtain $w_{z, 0}$. In order to avoid numerical difficulties near the boundaries $\partial \Omega$ and $\partial D$, which arise from the representation of the function $w$ by a combined layer potential, we chose an auxiliary integration domain $\Omega_{D, \varrho}$ with $\varrho>0$ as shown in Figure 7.2. Here $\Omega_{D, \varrho}$ is defined as the domain bounded by the surfaces

$$
\partial D_{\varrho}:=\{x-\varrho \nu(x): x \in \partial D\}, \quad \varrho>0,
$$

and

$$
\partial \Omega_{\varrho}:=\{x-\varrho \nu(x): x \in \partial \Omega\}, \quad \varrho>0,
$$

i.e. we exclude the "near-boundary" region around $\partial D$ and $\partial \Omega$ for integration and add a correction term for the contribution of this near-boundary region which will be explained later on. Then, we can calculate the gradient of $u$ in $\Omega_{D, \varrho}$ by applying the $\nabla$ operator to the kernel functions of the integral 

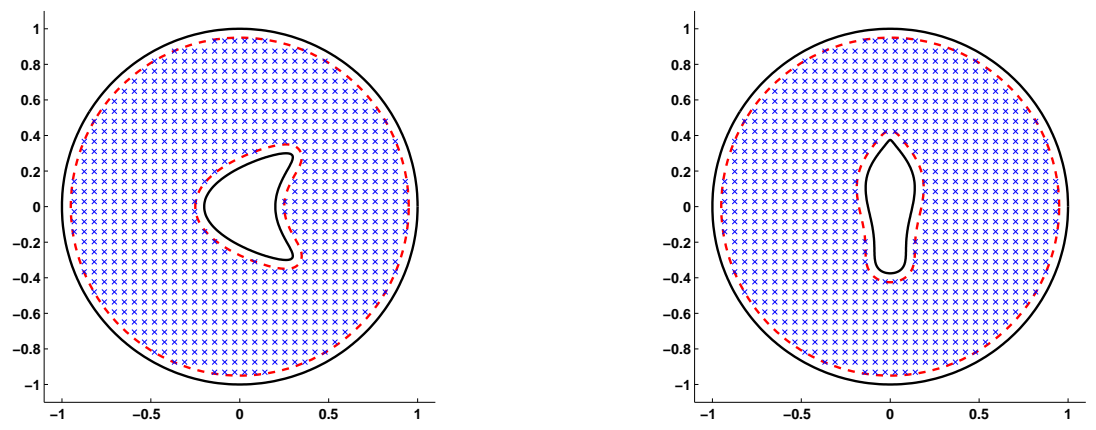

Fig. 7.2. The auxiliary integration domain $\Omega_{D, 0.05}$ for the obstacles of Setting 2 and 3 .

operators $S$ and $K$ of the combined layer potential. In the two-dimensional case, we obtain with $\Phi(x, y)=\frac{i}{4} H_{0}^{(1)}(\kappa|x-y|)$ and

$$
\frac{d}{d z} H_{n}^{(1)}(z)=\frac{1}{2}\left(H_{n-1}^{(1)}(z)-H_{n+1}^{(1)}(z)\right)
$$

the gradient

$$
\begin{aligned}
\nabla u(x)= & \int_{\Gamma_{1}} \nabla_{x} \frac{\partial \Phi(x, y)}{\partial \nu(y)} \varphi_{1}(y) d s(y)-i \eta \int_{\Gamma_{1}} \nabla_{x} \Phi(x, y) \varphi(y)_{1} d s(y) \\
& +\int_{\Gamma_{2}} \nabla_{x} \frac{\partial \Phi(x, y)}{\partial \nu(y)} \varphi_{2}(y) d s(y) \\
= & \int_{\Gamma_{1}} k_{1}(x, y) \varphi_{1}(y) d s(y)-i \eta \int_{\Gamma_{1}} k_{2}(x, y) \varphi_{1}(y) d s(y) \\
& +\int_{\Gamma_{2}} k_{1}(x, y) \varphi_{2}(y) d s(y)
\end{aligned}
$$

of $u$ for all $x \in \Omega_{D, \varrho}$ with the two kernel functions

$$
\begin{aligned}
k_{1}(x, y)=\frac{i \kappa}{4} & \left(\frac{\kappa}{2}\left(H_{0}^{(1)}(\kappa|x-y|)-H_{2}^{(1)}(\kappa|x-y|)\right)(x-y) \cdot \nu(y) \frac{x-y}{|x-y|^{2}}\right. \\
& \left.+H_{1}^{(1)}(\kappa|x-y|) \frac{|x-y|^{2} \nu(y)-(x-y) \cdot \nu(y)(x-y)}{|x-y|^{3}}\right)
\end{aligned}
$$

and

$$
k_{2}(x, y)=-\frac{i \kappa}{4} H_{1}^{(1)}(\kappa|x-y|) \frac{x-y}{|x-y|} .
$$

In the next section we give numerical examples for both formulations (7.31) and (7.41) of the Ikehata functional.

\subsection{Numerical Examples}

In the study of the PSM we have used the forward solver to validate the reconstruction Algorithms 6.8 and 6.17 for the reconstructed field. Since the 
probe method method does not reconstruct the field values we have to use another test for this method's reconstruction scheme. With the reformulation (7.41) of the indicator functional in Lemma 7.3 we can crosscheck the numerical results of the indicator function prior to the reconstruction of the obstacle.

As described in the previous section, we will have to omit the "nearboundary" region around $\partial D$ and $\partial \Omega$ when integrating $|w|^{2}$ and $|\nabla w|^{2}$ over $\Omega_{D}$. For the proper choice of the parameter $\varrho$ for the integration domain $\Omega_{D, \varrho} \subset \Omega_{D}$ we investigate the error between the true solution of the direct obstacle boundary value problem and the solution given by the forward solver together with their gradients, first. With $x_{i}=(0,0) \in D$ and $x_{e}=(0,1.5) \in \mathbb{R}^{2} \backslash \bar{\Omega}$ we define the following solution

$$
u^{*}(x):=\Phi\left(x, x_{i}\right)+\Phi\left(x, x_{e}\right)
$$

to the Helmholtz equation in $\Omega_{D}$ and compare it to the solution $u$ of the boundary value problem $(7.7)-(7.9)$ with the boundary values $f=\left.u^{*}\right|_{\partial \Omega}$ and $g=\left.u^{*}\right|_{\partial D}$ of the reference function $u^{*}$. The latter is governed with the integral equation method as described in Section 4.2 where we use the analytic differentiation of the kernels presented in the previous section for the evaluation of the gradient. Table $7.1-7.3$ show the error between the true solution $u^{*}$ and the calculated solution $u$ in the $C^{1}\left(\partial \Omega_{D, \varrho}\right)$ norm for various parameters $\varrho$ and quadrature points $n$ on the boundary $\partial D$ for Setting $1-3$. On the measurement boundary we choose $2 n$ quadrature points for the boundary integrals. For the numerical evaluation of the error we use the sampling points on the parallel surface $\partial \Omega_{D, \varrho}$ corresponding to the $3 n$ total quadrature points. The wave number for this numerical experiment is $\kappa=2$.

\begin{tabular}{cccccc}
\hline \hline$\varrho$ & $n=64$ & $n=128$ & $n=256$ & $n=512$ & $\left\|u^{*}\right\|_{C^{1}\left(\partial \Omega_{D, \varrho}\right)}$ \\
\hline 0.1 & 0.0001045 & 0.00000003 & 0.00000002 & 0.00000001 & 1.44719954 \\
0.08 & 0.0016772 & 0.00000008 & 0.00000002 & 0.00000001 & 1.51228016 \\
0.06 & 0.0254264 & 0.00001845 & 0.00000002 & 0.00000001 & 1.58540300 \\
0.04 & 0.3683504 & 0.00391674 & 0.00000023 & 0.00000001 & 1.66847309 \\
0.02 & 5.7974287 & 0.75444512 & 0.00845911 & 0.00000054 & 1.76451802 \\
\hline \hline
\end{tabular}

Table 7.1. The error between the true solution $u^{*}$ and the calculated solution $u$ measured in the $C^{1}$-norm for a different number of sampling points $n$ on parallel surfaces in distance $\varrho$ from the obstacle of Setting 1.

Following this numerical example we chose $n=256$ sampling points on the interior and $2 n=512$ points on the exterior boundary of the domain $\Omega_{D}$ in the combined layer approach of the forward solver to obtain an error below $1 \%$ in $\Omega_{D, \varrho}$ with $\varrho=0.03$. Figure 7.3 illustrates the behaviour of the error measured in the $L^{2}$-norm against the distance parameter $\varrho$ exemplarily for the obstacle of Setting 2 and $n=256$ quadrature points on $\partial D$. This 


\begin{tabular}{cccccc}
\hline \hline$\varrho$ & $n=64$ & $n=128$ & $n=256$ & $n=512$ & $\left\|u^{*}\right\|_{C^{1}\left(\partial \Omega_{D, \varrho}\right)}$ \\
\hline 0.1 & 0.00011887 & 0.00000005 & 0.00000003 & 0.00000001 & 1.60651833 \\
0.08 & 0.00168121 & 0.00000009 & 0.00000003 & 0.00000001 & 1.68612447 \\
0.06 & 0.02542637 & 0.00001845 & 0.00000003 & 0.00000002 & 1.77653129 \\
0.04 & 0.36835037 & 0.00391674 & 0.00000023 & 0.00000002 & 1.88058009 \\
0.02 & 5.87401837 & 0.75444512 & 0.00845911 & 0.00000055 & 2.00282798 \\
\hline \hline
\end{tabular}

Table 7.2. The error between the true solution $u^{*}$ and the calculated solution $u$ measured in the $C^{1}$-norm for a different number of sampling points $n$ on parallel surfaces in distance $\varrho$ from the obstacle of Setting 2 .

\begin{tabular}{cccccc}
\hline \hline$\varrho$ & $n=64$ & $n=128$ & $n=256$ & $n=512$ & $\left\|u^{*}\right\|_{C^{1}\left(\partial \Omega_{D, \varrho}\right)}$ \\
\hline 0.1 & 0.00010455 & 0.00000006 & 0.00000003 & 0.00000002 & 1.64296922 \\
0.08 & 0.00167721 & 0.00000009 & 0.00000003 & 0.00000002 & 1.74284684 \\
0.06 & 0.02542642 & 0.00001845 & 0.00000004 & 0.00000002 & 1.86208934 \\
0.04 & 0.36835043 & 0.00391674 & 0.00000023 & 0.00000002 & 2.00762486 \\
0.02 & 6.57761161 & 0.75444512 & 0.00845911 & 0.00000054 & 2.19128268 \\
\hline \hline
\end{tabular}

Table 7.3. The error between the true solution $u^{*}$ and the calculated solution $u$ measured in the $C^{1}$-norm for a different number of sampling points $n$ on parallel surfaces in distance $\varrho$ from the obstacle of Setting 3 .
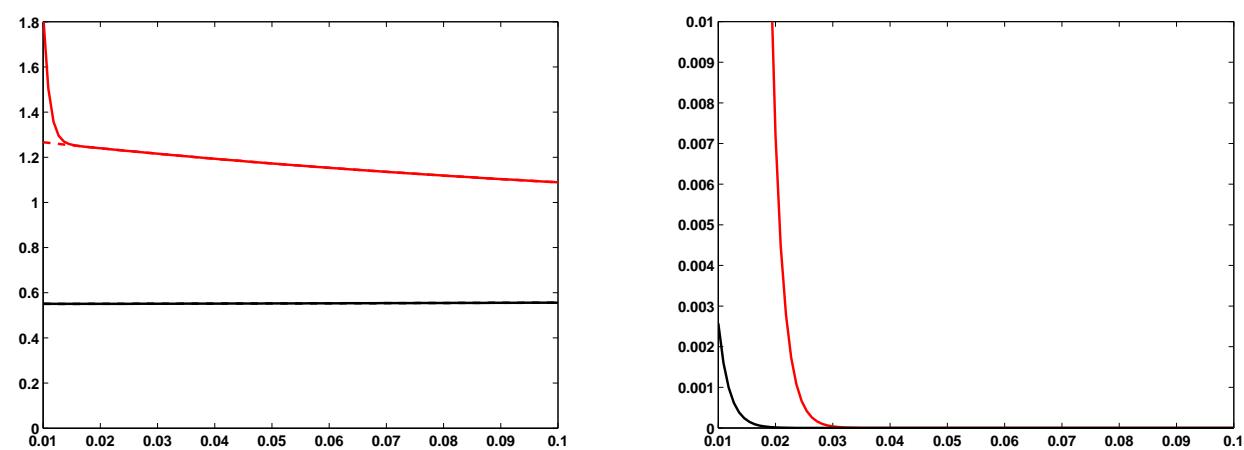

Fig. 7.3. The $L^{2}$-norm $\sqrt{\int_{\partial \Omega_{D, \ell}}|u|^{2} d x}$ of the calculated solution (black) and the $L^{2}$-norm $\sqrt{\int_{\partial \Omega_{D, \varrho}}\|\nabla u\|^{2} d x}$ of its gradient (red) is plotted together with the corresponding $L^{2}$-norms of the true solution $u^{*}$ (dashed lines) in the left figure. The right figure shows the absolute error between $u$ and $u^{*}$ (black) and their gradients (red) measured in the $L^{2}$-norm.

example demonstrates that we cannot use a smaller $\varrho$ since the error in the evaluation of the potential layers close to the boundary blows off. However, the norm of $w_{z}$ and $\nabla w_{z}$ will become unbounded when $z$ tends to the boundary $\partial D$. Simply omitting the near-boundary region between $\partial D_{\varrho}$ and $\partial D$ will therefore result in a significant error in (7.41). Hence we will add a correcting term for the integral over $\Omega_{D} \backslash \Omega_{D, \varrho}$ which is given by a quadrature of $w_{z}$ and $\nabla w_{z}$, respectively, using the boundary values on $\partial \Omega_{D, \varrho}$. We apply the quadrature rule 


$$
Q_{n}(w)=\sum_{j=1}^{n} \alpha_{j}^{(n)} w\left(x_{j}^{(n)}\right), \quad n \in \mathbb{N},
$$

where the quadrature points $x_{j}^{(n)}, j=1, \ldots, n$ are given by a discrete parametrization of the parallel curve $\partial \Omega_{D, \varrho}$ and the weights $\alpha_{j}^{(n)}, j=$ $1, \ldots, n$ are obtained as the product of the distance $\varrho$ with the corresponding arc length elements. For our purpose to compare the two formulations (7.31) and (7.41) this cut-off scheme together with the correction term yields a sufficient accuracy which is demonstrated by the following numerical examples.

For the comparison of these two formulations we calculate the Ikehata functional with formula (7.41) and (7.31), respectively. Additionally, we investigate the influence of the point source approximation by studying (7.41), where we insert the point source $v_{z, 0}$ and the solution $w_{z, 0}$ to the boundary value problem (7.7) - (7.9) with boundary values $f=0$ and $g=-\left.\Phi(\cdot, z)\right|_{\partial D}$ to obtain the limiting function $\hat{I}$. For the correction term we use $n=192$ quadrature points on $\partial \Omega_{D, \varrho}$. The point source is approximated on circular approximation domains of radius $r=1$ in distance 0.1 from the source using the regularization parameter $\alpha=10^{-8}$. Furthermore, we apply strategy (5.109) where we assume the knowledge of the interior point $0 \in D$. Then Figure 7.4 summarizes the numerical results for the ellipse whereas Figure 7.5 illustrates the behaviour of the indicator function in the different formulations for the kite and the boat.

A comparison of the indicator function evaluated with the domain integral formulation (7.41) given in the second row and with the boundary integral formulation (7.31) presented in the third row validates the implementation of the reconstruction algorithm. In particular we see from Figure 7.4 that the error between these two formulations is less than $3 \%$. We also observe that the limiting function $\hat{I}$ shown in the first row of Figure 7.5 gives a good characterization of the obstacle which cannot be achieved with the simple circular approximation domains as indicated in the second and third row. Nevertheless, we can expect similar improvements like for the point source method if we adapt the approximation domain to the geometry of the scatterer. Once again the indicator function $\mathcal{I}_{\text {enl }}$ for the enlighted area which is plotted in the last row of Figure 7.5 provides us with a first guess about the scatterer's shape. Hence we apply a second run of the point source method with the adapted approximation domains of Figure 6.9 and Figure 6.12. With the parameters $\alpha=10^{-7}, \tau=40$ and $r=0.3$ we obtain an improved reconstruction of the obstacles as illustrated in Figure 7.6. This significant improvement of the reconstructions compared with the point source method is due to the fact that we use more input data in the reconstruction scheme.

In this chapter we presented a numerical realization of the probe method, which is based on the techniques developed for the point source method in 

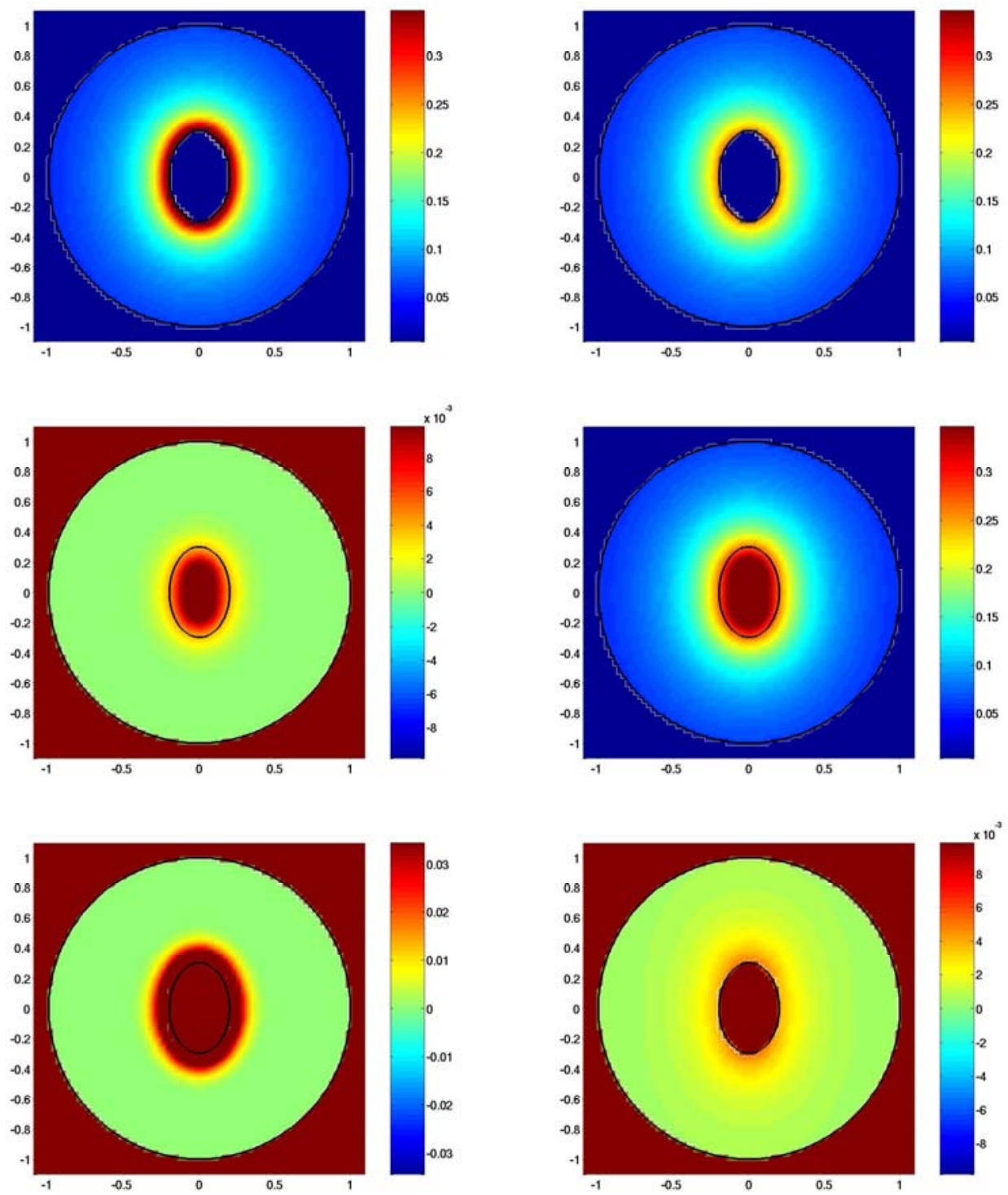

Fig. 7.4. Top left: the limiting indicator functional $\hat{I}$. Top right: indicator function computed with formula (7.41). Middle left: the indicator function $\mathcal{I}_{\text {enl }}$ of the enlighted area. Middle right: the indicator function evaluated with the D-t-N formulation (7.31). Bottom left: modulus of the difference between the limiting indicator function and the D-t- $\mathrm{N}$ formulation. Bottom right: modulus of the difference between the $\mathrm{D}-\mathrm{t}-\mathrm{N}$ formulation and the domain integral formulation (7.41). 

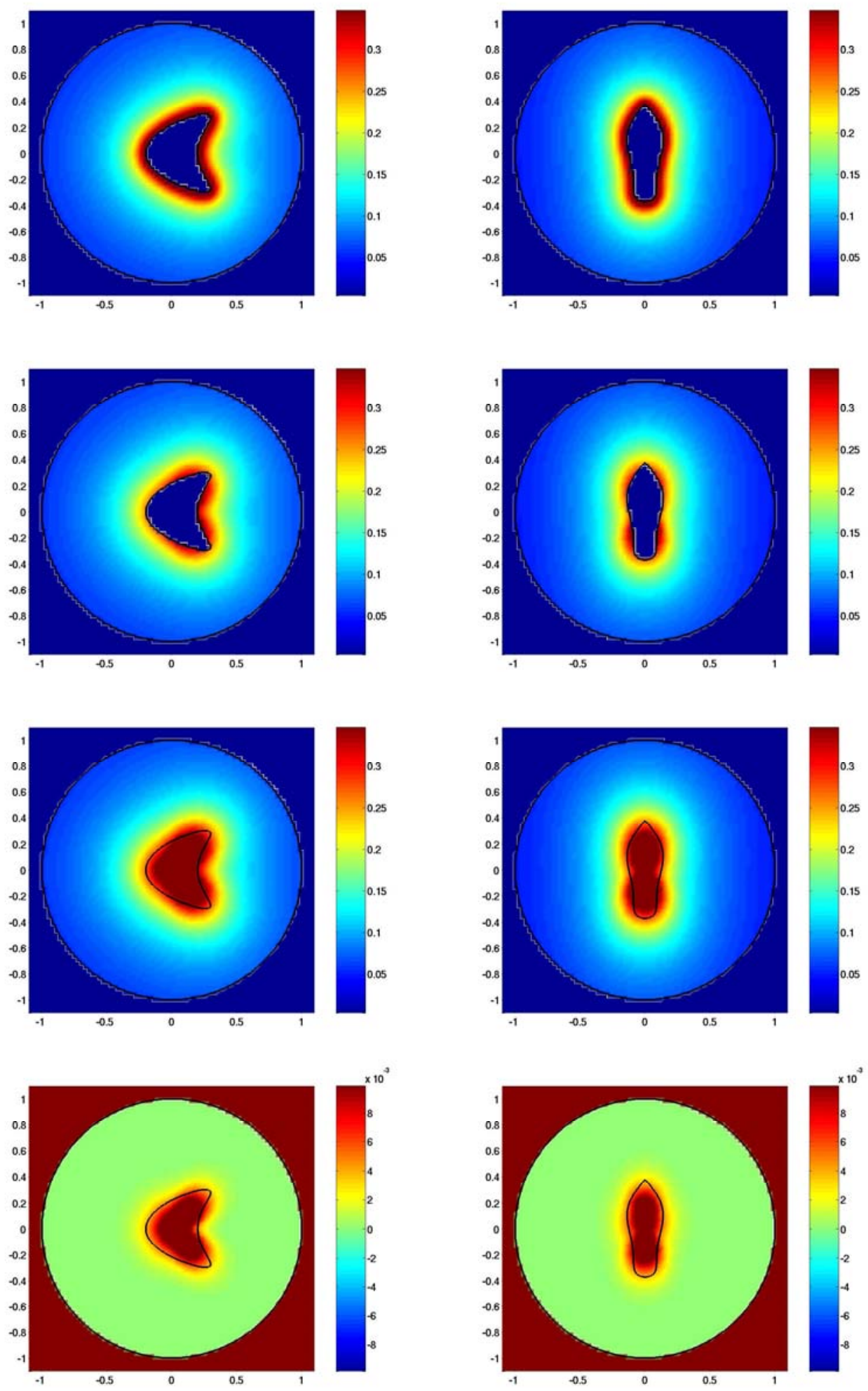

Fig. 7.5. The columns show the indicator functional in several formulations for the kite and the boat, respectively. The first row illustrates the limiting indicator function $\hat{I}$ while the second row shows the indicator function computed with formula (7.41). The indicator function plotted in the third row was evaluated with the D-t-N formulation (7.31). Finally, the last row shows the indicator function $\mathcal{I}_{\text {enl }}$ for the enlighted area. 

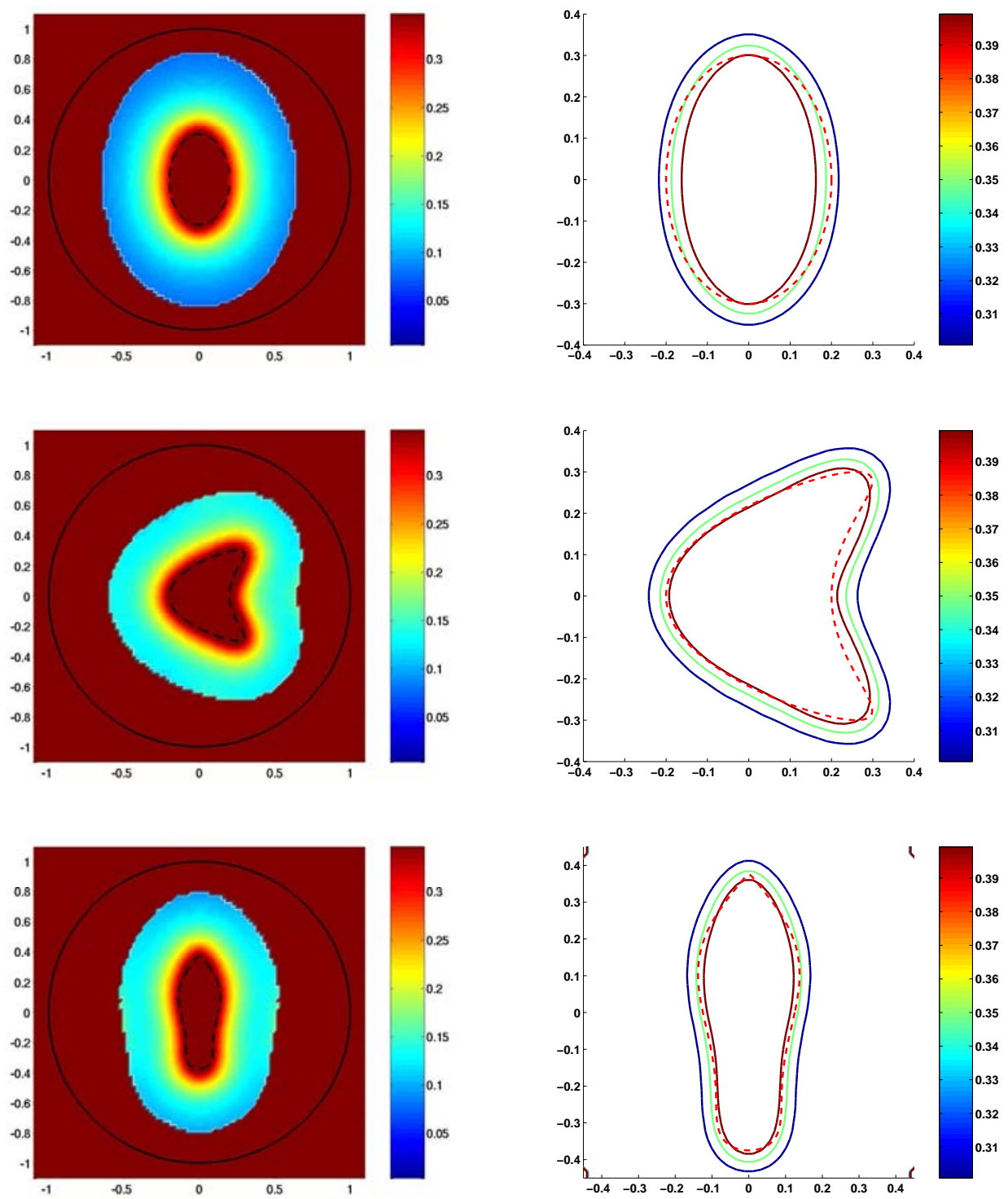

Fig. 7.6. The indicator function computed with the adapted approximation domains is plotted for the obstacles of Setting $1-3$. In the left column the isolines of the levels $0.3,0.35,0.4$ are indicated. The obstacle is marked with a dashed line. The wave number in this example was $\kappa=2.0$.

the previous chapter. In particular, we modified the two-step algorithm in a straightforward way to obtain a reconstruction algorithm for the probe method. In order to validate this algorithm we also implemented the domain integral formulation (7.41) numerically and compared it with the D-t-N formulation (7.31) and the limiting indicator function $\hat{I}$. This comparison, which is illustrated in Figure 7.4 and Figure 7.5, demonstrates that the numerical implementation of the probe method in the D-t-N formulation and in its domain integral formulation coincide. Furthermore, this numeri- 
cal study shows that the limiting indicator function $\hat{I}$ is approximated by the D-t-N formulation. We can also deduce that the quality of this approximation depends on the choice of the approximation domains. In particular, the circular approximation domains yield a better approximation of the limiting indicator function in case of the convex ellipse than in the case of the nonconvex boat- and kite-shaped obstacles. As for the point source method, adaptively chosen approximation domains improve the reconstruction significantly. Compared with the the point source method, the overall reconstructions of the obstacles with the probe method yield a more accurate boundary reconstruction but the probe method needs more input data for the reconstruction algorithm.

While the point source method was capable in reconstructing an obstacle from one set of Cauchy data on the measurement boundary $\partial \Omega$, the probe method needs the knowledge of the full Dirichlet-to-Neumann map on $\partial \Omega$. However, information about the type of boundary condition is not needed by the probe method. We will focus on reconstruction schemes that are based on the knowledge of the far field patterns of all incident plane waves but do not need information on the physical properties of the scatterer in the final chapter of this work. 



\section{The Singular Sources Method}

In this chapter we want to compare the reconstruction schemes that are based on point source approximation techniques with a different type of reconstruction methods - the linear sampling and the factorization method - of inverse scattering. To this end we first introduce the singular sources method which was originally formulated for the inverse scattering problem while the formulation of the probe method was formulated for the inverse acoustic boundary value problem. Then, we compare the singular sources method with the linear sampling and the factorization method and study their stability with respect to data noise in the two-dimensional inverse acoustic problem setting. Furthermore, we provide numerical examples of the singular sources method in the inverse three-dimensional acoustic scattering problem.

\subsection{The SSM in Inverse Scattering}

We introduce the singular sources method as proposed in [54] by Potthast for the inverse acoustic scattering problem. It is motivated by the uniqueness result of Isakov [26] for the transmission problem and the simplification thereof presented by Kirsch and Kress in [30]. In this paper the authors transfer the ideas of Isakov to prove uniqueness for the inverse Neumann problem. A crucial ingredient in this proof is the singular behaviour of the scattered field $\Phi^{s}(y, z)$ of an incident point source $\Phi(\cdot, z)$ in a point $y$ on the boundary $\partial D$ of the scatterer when the source point $z$ tends to $y$.

The method of singular sources uses this behaviour to define an indicator function that is an approximation to $\Phi^{s}(z, z)$ in the admissibility region $\mathcal{A}$ defined in (6.31). For the evaluation of the indicator function the singular sources method reconstructs the scattered field $\Phi^{s}(\cdot, z)$ in the source point $z$ using the point source method in a multi-wave setting. Then the boundary $\partial D$ of an obstacle $D$ is determined as the set of points where the reconstructed scattered field $\Phi^{s}(z, z)$, i.e. the indicator function becomes singular. In [54] Potthast applied the singular sources method (SSM) to the inverse acoustic scattering problem using the mixed reciprocity relation and 
the same approximation techniques as for the PSM. In particular, he defined a sequence of backprojection operators $Q_{\varepsilon}: L^{2}\left(\mathbb{S}^{m-1} \times \mathbb{S}^{m-1}\right) \longrightarrow L^{\infty}(\mathcal{B} \times \mathcal{B})$ via

$$
\left(Q_{\varepsilon_{x}} w\right)(x, z):=\frac{1}{\gamma_{m}} \int_{\mathbb{S}^{m-1}} \int_{\mathbb{S}^{m-1}} g_{x, \varepsilon}(d) g_{z, \tilde{\varepsilon}}(\hat{x}) w(-d, \hat{x}) d s(d) d s(\hat{x})
$$

with the densities $g_{x, \varepsilon}, g_{z, \tilde{\varepsilon}} \in L^{2}\left(\mathbb{S}^{m-1}\right)$ given as the solution of the regularized equation (5.82) with appropriately chosen approximation levels $\varepsilon, \tilde{\varepsilon}$ and $\gamma_{m}$ defined in (6.84). Each backprojection operator $Q_{\varepsilon}$ maps the far field pattern of all incident plane waves to an approximation of the scattered field $\Phi^{s}(x, z)$ on the admissibility region $\mathcal{A}$. Then, this approximation is evaluated in the source point $x=z$ of the incident point wave and provides an indicator function $\tilde{I}(z)=\left(Q_{\varepsilon} u^{\infty}\right)(z, z)$ approximating $\Phi^{s}(z, z)$ on $\mathcal{A}$. We remark that the backprojection operator $Q_{\varepsilon}$ can formally be factorized into a product $P \circ M \circ P$ of the point source method $P$ and the mixed reciprocity operator $M$. The latter maps the scattered field $u^{s}(z, d)$ of an incident plane wave with direction of incidence $d \in \mathbb{S}^{m-1}$ to the far field pattern $\Phi^{\infty}(-d, z)$ of a scattered wave due to the incident point source $\Phi(\cdot, z)$.

In the following we give a detailed overview of the singular sources method and present a reformulation for the inverse acoustic boundary value problem. To this end we first summarize the results on the behaviour of the scattered field for singular incident fields in the next theorem.

Theorem 8.1. Consider the scattering of a point source $\Phi(\cdot, z)$ by a soundsoft scatterer $D \subset \mathbb{R}^{2}$. Then there exist constants $\tau, c, C, E>0$ such that the scattered field satisfies the lower estimate

$$
\left|\Phi^{s}(z, z)\right| \geq c|\log d(z, D)|
$$

in the strip $0<d(z, D)<\tau$ and the upper estimate

$$
\left|\Phi^{s}(z, z)\right| \leq C|\log d(z, D)|+E
$$

for all $z \in \mathcal{B} \backslash \bar{D}$.

In $\mathbb{R}^{3}$ there exist constants $\tau, c, C>0$ such that the lower estimate

$$
\left|\Phi^{s}(z, z)\right| \geq \frac{c}{|d(z, D)|}
$$

holds in the tube $0<d(z, D)<\tau$ and we have the upper estimate

$$
\left|\Phi^{s}(z, z)\right| \leq \frac{C}{|d(z, D)|}
$$

for all $z \in \mathcal{B} \backslash \bar{D}$. 
The proof of this theorem can be found in [57], Theorem 2.1.15. It proves that the function

$$
I(z):=\left|\Phi^{s}(z, z)\right|, \quad z \in \mathcal{B} \backslash \bar{D}
$$

is bounded in every set $\mathcal{B}_{\tau}$ of the form

$$
\mathcal{B}_{\tau}:=\{z \in \mathcal{B} \backslash \bar{D}: d(z, D)>\tau\}, \quad \tau>0
$$

but unbounded when $z$ tends to the obstacle's boundary, i.e.

$$
\lim _{z \rightarrow \partial D} I(z)=\infty
$$

holds. Thus the function $I$ may serve as an indicator function for the reconstruction of the obstacle $D$. However, we cannot calculate the indicator function $I(z)=\Phi^{s}(z, z)$ directly without the knowledge of the obstacle $D$ and the boundary condition on $\partial D$. Therefore we will compute an approximation for the scattered field $\Phi^{s}(\cdot, z)$ in the source point $z \in \mathcal{B} \backslash \bar{D}$ based on the ideas of the point source method. We recall that the reconstruction formula (6.88) of the PSM provides an approximation of the scattered wave by the knowledge of its far field pattern which neither depends on the type of incident field nor on the type of boundary condition posed on $\partial D$. Hence, an application of the PSM in this multi-wave setting, that means simultaneously, to the far field patterns $u^{\infty}(\cdot, d), d \in \mathbb{S}^{m-1}$, is justified. This yields approximations to the scattered fields $u^{s}(\cdot, d)$ for all incident directions $d \in \mathbb{S}^{m-1}$ of the incident plane waves $u^{i}(\cdot, d)$. We recall the mixed reciprocity relation

$$
\Phi^{\infty}(-d, z)=\gamma_{m} u^{s}(z, d), \quad z \in \mathbb{R}^{m} \backslash \bar{D}, d \in \mathbb{S}^{m-1},
$$

where $\gamma_{m}$ is defined in (6.84), see Theorem 2.2.4 in [54]. The mixed reciprocity relation sets up a correspondence between the values of the simultaneously reconstructed scattered fields $u^{s}(z, \cdot)$ and the far field pattern $\Phi^{\infty}(\cdot, z)$ of the scattered point source $\Phi(\cdot, z)$. Note that the observation direction of the far field pattern $\Phi^{\infty}(\cdot, z)$ is directly opposed to the incident direction of the corresponding plane wave. Nevertheless, we are given the full far field pattern of the scattered field $\Phi^{s}(\cdot, z)$ which is then approximately reconstructed with a second application of the one-wave PSM to $\Phi^{\infty}(\cdot, z)$. We settle this idea in the following without an explicit application of the mixed reciprocity relation. We restrict ourselves to a sound-soft boundary condition on $\partial D$ in order to focus on the basic idea of the proof.

For $z \in \mathcal{B} \backslash \bar{D}$ we choose an approximation domain $G$ with $z \notin \bar{G}$ which is large enough to contain the closure of the unknown scatterer $D$ in its interior. Then, we construct a density $g_{z, \varepsilon}$ such that the superposition of plane waves $v_{z, \varepsilon}$ defined by (5.44) approximates the point source $\Phi(\cdot, z)$ on $G$ in the sense (5.37). This can be obtained by solving the ill-posed integral equation 


$$
\int_{\mathbb{S}^{m-1}} e^{i \kappa y \cdot \hat{x}} g_{z, \varepsilon}(\hat{x}) d s(\hat{x})=\Phi(y, z), \quad y \in \partial G
$$

with a Tikhonov regularization scheme (5.82) as demonstrated in Chapter 5. By the sound-soft boundary condition and the property (5.37) of the Herglotz wave function $v_{z, \varepsilon}$ we obtain the approximation

$$
\begin{gathered}
\left\|\Phi^{s}(\cdot, z)-\int_{\mathbb{S}^{m-1}} u^{s}(\cdot, \hat{x}) g_{z, \varepsilon}(\hat{x}) d s(\hat{x})\right\|_{C^{1}(\partial D)} \\
\left\|\Phi(\cdot, z)-\int_{\mathbb{S}^{m-1}} u^{i}(\cdot, \hat{x}) g_{z, \varepsilon}(\hat{x}) d s(\hat{x})\right\|_{C^{1}(\partial D)} \leq \varepsilon
\end{gathered}
$$

for the scattered field $\Phi^{s}(\cdot, z)$ on $\partial D$ for all $z$ in an enlighted area $\mathcal{E}$. We recall from Chapter 6 that an enlighted area ensures a uniform approximation of the fundamental solution on the obstacle $D$. In particular we point out that any compact subset of the admissibility region $\mathcal{A}$ defines an enlighted area. Let $\mathcal{E}$ be a compact subset of $\mathcal{A}$. Then $\mathcal{E}$ is also a compact subset of $\mathcal{B} \backslash \bar{D}$ and the well-posedness of the exterior Dirichlet problem, see Theorem 3.9 in [7], provides the estimate

$$
\left|\Phi^{s}(x, z)-\int_{\mathbb{S}^{m-1}} u^{s}(x, \hat{x}) g_{z, \varepsilon}(\hat{x}) d s(\hat{x})\right| \leq c_{1} \varepsilon
$$

for all $x, z \in \mathcal{E}$ and a constant $c_{1}>0$. Furthermore, for any given $\tilde{\varepsilon}>0$ the point source method (6.88) constructs densities $\tilde{g}_{x, \tilde{\varepsilon}}$ such that

$$
\sup _{\hat{x} \in \mathbb{S}^{m-1}}\left|u^{s}(x, \hat{x})-\frac{1}{\gamma_{m}} \int_{\mathbb{S}^{m-1}} u^{\infty}(-d, \hat{x}) \tilde{g}_{x, \tilde{\varepsilon}}(d) d s(d)\right| \leq \tilde{\varepsilon}
$$

for all $x \in \mathcal{E}$.

Finally, we apply the triangle inequality using (8.12) and (8.13) to obtain the estimate

$$
\begin{aligned}
& \left|\Phi^{s}(x, z)-\int_{\mathbb{S}^{m-1}}\left(\frac{1}{\gamma_{m}} \int_{\mathbb{S}^{m-1}} u^{\infty}(-d, \hat{x}) \tilde{g}_{x, \tilde{\varepsilon}}(d) d s(d)\right) g_{z, \varepsilon}(\hat{x}) d s(\hat{x})\right| \\
\leq & \left|\Phi^{s}(x, z)-\int_{\mathbb{S}^{m-1}} u^{s}(x, \hat{x}) g_{z, \varepsilon}(\hat{x}) d s(\hat{x})\right| \\
& +\left|\int_{\mathbb{S}^{m-1}}\left(u^{s}(x, \hat{x})-\frac{1}{\gamma_{m}} \int_{\mathbb{S}^{m-1}} u^{\infty}(-d, \hat{x}) \tilde{g}_{x, \tilde{\varepsilon}}(d) d s(d)\right) g_{z, \varepsilon}(\hat{x}) d s(\hat{x})\right| \\
\leq & c_{1} \varepsilon+c_{2} \tilde{\varepsilon}\left\|g_{z, \varepsilon}\right\|_{L^{2}\left(\mathbb{S}^{m-1}\right)}
\end{aligned}
$$

with constants $c_{1}, c_{2}>0$. Obviously, we cannot improve the approximations (8.12) and (8.13) independently in order to decrease the right hand side of this estimate since $\left\|g_{z, \varepsilon}\right\|$ becomes unbounded when $\varepsilon \rightarrow 0$. Therefore, for any $\varepsilon>0$ we choose the densities $g_{z, \varepsilon}$ such that $(8.12)$ is satisfied, first. For the strategy (5.109) of orthogonal translations of a fixed reference 
configuration $\left(0, G_{0}\right)$ the norm $\left\|g_{z, \varepsilon}\right\|=c_{\varepsilon}$ is constant for all $z \in \mathcal{E}$. In general, $\left\|g_{z, \varepsilon}\right\|$ depends on the choice of the approximation domain and we have to ensure the boundedness of $\left\|g_{z, \varepsilon}\right\| \leq c_{\varepsilon}$. Then we choose $\tilde{g}_{x, \tilde{\varepsilon}}$ such that (8.13) holds with

$$
\tilde{\varepsilon}:=\frac{\varepsilon}{c_{\varepsilon}} .
$$

With this choice of the approximation levels $\varepsilon$ and $\tilde{\varepsilon}$ the backprojected far field pattern

$$
\left(Q_{\varepsilon} u^{\infty}\right)(x, z)=\frac{1}{\gamma_{m}} \int_{\mathbb{S}^{m-1}} \int_{\mathbb{S}^{m-1}} u^{\infty}(-d, \hat{x}) \tilde{g}_{x, \tilde{\varepsilon}}(d) d s(d) g_{z, \varepsilon}(\hat{x}) d s(\hat{x})
$$

satisfies the estimate

$$
\left|\Phi^{s}(x, z)-\left(Q_{\varepsilon} u^{\infty}\right)(x, z)\right| \leq\left(c_{1}+c_{2}\right) \varepsilon,
$$

uniformly for all $(x, z) \in \mathcal{E} \times \mathcal{E}$. Hence, we have proven the following convergence theorem.

Theorem 8.2. (SSM for inverse acoustic scattering problem.) Let $\mathcal{E}$ be a compact subset of the admissibility region $\mathcal{A}$ and let $\mathcal{G}$ be a strategy for the choice of the approximation domain such that for every $\varepsilon>0$ the densities $g_{z, \varepsilon} \in L^{2}\left(\mathbb{S}^{m-1}\right)$ are uniformly bounded $\left\|g_{z, \varepsilon}\right\| \leq c_{\varepsilon}$. Then, the backprojected far field pattern (8.16) converges uniformly towards $\Phi^{s}(x, z)$ on the cartesian product $\mathcal{E} \times \mathcal{E}$ of the enlighted area. The densities $g_{z, \varepsilon}$ and $\tilde{g}_{x, \tilde{\varepsilon}}$ are obtained by solving the ill-posed linear integral equation (8.10) such that (8.12), (8.13) and (8.15) are satisfied.

In particular, the approximating indicator function

$$
\tilde{I}(z):=\frac{1}{\gamma_{m}} \int_{\mathbb{S}^{m-1}} \int_{\mathbb{S}^{m-1}} u^{\infty}(-d, \hat{x}) \tilde{g}_{z, \tilde{\varepsilon}}(d) d s(d) g_{z, \varepsilon}(\hat{x}) d s(\hat{x}), \quad z \in \mathcal{B},
$$

which is given by the backprojected far field pattern $Q_{\varepsilon} u^{\infty}$ evaluated in $(z, z) \in \mathcal{B} \times \mathcal{B}$, approximates the true indicator function $I(z)=\Phi^{s}(z, z)$ whenever $z \in \mathcal{B}$ is contained in a compact subset of the admissibility region $\mathcal{A}$. Then, we obtain an approximation to the unknown scatterer $D$ as the set of points where $\tilde{I}(z)$ becomes larger than a predefined cut-off value $c_{0}$. This threshold value $c_{0}$ can be chosen empirically by investigating some reference obstacle $D_{0}$ with known boundary and simulated far field pattern $u_{0}^{\infty}(\cdot, \cdot)$. We will demonstrate the feasibility of this approach for the choice of the cut-off value when we present numerical examples. In contrast to the point source method the singular sources method needs to know the full far field pattern for all incident waves as input data. This means that we must have more information available than in the single wave setting but we also expect a better quality of the reconstructions due to the increase in information. On the other hand, the SSM reconstructs a scatterer without 
the knowledge of the boundary condition on $\partial D$ while the point source method essentially needs this information in the reconstruction algorithm. Hence the SSM is applicable in a more general setting.

As for the PSM we need an approximation to the admissibility region $\mathcal{A}$ to decide where the indicator function (8.18) of the SSM yields a reliable approximation to $\Phi^{s}(z, z)$. To this end we define the indicator function

$$
\mathcal{I}_{\text {enl }}(z)=\left(Q_{\varepsilon_{1}} u^{\infty}-Q_{\varepsilon_{2}} u^{\infty}\right)(z, z), \quad z \in \mathcal{S}
$$

of the admissibility region, which is constructed as the difference of two backprojected far field patterns in $(z, z)$ obtained with different approximation levels $\varepsilon_{1}, \varepsilon_{2}$. In the admissibility region $\mathcal{A}$ this difference will be small in view of Theorem 8.2 while it amplifies the oscillations of the Herglotz wave function outside of its domain of approximation. Finally, we remark that the SSM is essentially based on the point source approximation so that we can apply the same techniques that have already been developed in the previous chapters. In particular the calculation of the densities $g_{z, \varepsilon}$ and $\tilde{g}_{z, \tilde{\varepsilon}}$ defining the backprojection operator $Q_{\varepsilon}$ can be made efficient by considering translations of appropriate reference configurations $\left(0, G_{0}\right)$. We summarize the singular sources method in the following algorithm.

Definition 8.3 (Singular sources method for inverse scattering). For the inverse acoustic scattering problem the method of singular sources calculates an approximation $\tilde{I}$ to the true indicator function $I$ on subsets $\mathcal{E}_{c}$ of $\mathcal{B}$ by the following steps.

1. Choose a fixed reference configuration $\left(0, G_{0}\right)$ such that $0 \notin G_{0}$ and calculate the density $g_{0, \varepsilon} \in L^{2}\left(\mathbb{S}^{m-1}\right)$ and $\tilde{g}_{0, \tilde{\varepsilon}} \in L^{2}\left(\mathbb{S}^{m-1}\right)$ as a solution of equation (5.89) with $H$ defined by (5.45).

2. For each $z \in \mathcal{S} \subset \mathcal{B}$ choose a domain of approximation $G(z)$ of the form (5.86), i.e. the configuration $(z, G(z))$ is an orthogonal translation of the reference configuration $\left(0, G_{0}\right)$.

3. For each $z \in \mathcal{S}$ calculate the densities $g_{z, \varepsilon}, \tilde{g}_{z, \tilde{\varepsilon}} \in L^{2}\left(\mathbb{S}^{m-1}\right)$ via (5.91).

4. Calculate the indicator function $\tilde{I}(z)$ of the $S S M$ via (8.18).

5. Calculate an approximation $\mathcal{E}_{c}:=\left\{z \in \mathcal{S}:\left|\mathcal{I}_{\text {enl }}(z)\right|<c\right\}$ for the admissibility region using (8.19).

6. Repeat steps 1 - 5 with a different choice of the approximation domains until the union of the domains $\mathcal{E}_{c}$ is a doubly connected domain. Extend the single indicator functions $\tilde{I}$ with a suitable weighted mean operation to this domain.

7. Choose some suitable cut-off constant $C>0$ and decide

a) $z \in D$ if $|\tilde{I}(z)|>C$,

b) $z \in \mathbb{R}^{m} \backslash \bar{D}$ if $|\tilde{I}(z)| \leq C$,

for all $z \in \mathcal{E}_{c}$.

From a practical point of view it is often desired to work with incident point waves instead of incident plane waves and therefore we will present 
an algorithm for the SSM that fits these practical needs. We remark that we deduced the singular sources method without using the mixed reciprocity relation. The restriction to the scattering of incident plane waves in the previous setting is due to (8.11), where we have used the Herglotz wave functions, i.e. a superposition of plane waves, for the approximation of the point source. Now, we can make use of this fact to provide a reformulation of the SSM which is based on the scattering of incident point sources.

As we have seen in Chapter 5 we can also use a single-layer potential defined on the boundary $\partial \Omega$ for the approximation of the point source, see Theorem 5.7. In this case the estimate (8.11) has to be replaced by

$$
\begin{aligned}
& \left\|\Phi^{s}(\cdot, z)-\int_{\partial \Omega} \Phi^{s}(\cdot, \xi) g_{z, \varepsilon}(\xi) d s(\xi)\right\|_{C^{1}(\partial D)}= \\
& \left\|\Phi(\cdot, z)-\int_{\partial \Omega} \Phi(\cdot, \xi) g_{z, \varepsilon}(\xi) d s(\xi)\right\|_{C^{1}(\partial D)} \leq \varepsilon,
\end{aligned}
$$

and the estimate $(8.13)$ now reads as

$$
\sup _{\xi \in \partial \Omega}\left|\Phi^{s}(x, \xi)-\frac{1}{\gamma_{m}} \int_{\mathbb{S}^{m-1}} \Phi^{\infty}(-d, \xi) \tilde{g}_{x, \tilde{\varepsilon}}(d) d s(d)\right| \leq \tilde{\varepsilon}
$$

for all $x \in \mathcal{E}$. Here $\Phi^{\infty}(\cdot, \xi)$ denotes the far field pattern of an incident point source $\Phi(\cdot, \xi)$ with source point $\xi$ located on the boundary $\partial \Omega$. For the approximation of the scattered wave $\Phi^{s}(\cdot, \xi)$ in $(8.21)$ we use the PSM with Herglotz wave functions of the form (5.44), where the density $\tilde{g}_{x, \tilde{\varepsilon}}$ is obtained as the solution to the regularized integral equation (7.39). With the same argumentation as above we derive the indicator function

$$
\tilde{I}(z):=\frac{1}{\gamma_{m}} \int_{\partial \Omega} \int_{\mathbb{S}^{m-1}} \Phi^{\infty}(-d, \xi) \tilde{g}_{z, \tilde{\varepsilon}}(d) d s(d) g_{z, \varepsilon}(\xi) d s(\xi), \quad z \in \mathcal{B}
$$

of the SSM when the knowledge of the far field pattern $\Phi^{\infty}(\cdot, \xi)$ is given for all incident point sources located on the boundary $\partial \Omega$. Note that the densities $\tilde{g}_{z, \tilde{\varepsilon}} \in L^{2}\left(\mathbb{S}^{m-1}\right)$ and $g_{z, \varepsilon} \in L^{2}(\partial \Omega)$ are defined on different function spaces. The strategy to use translated reference configurations therefore only reduces the computational cost for calculating the densities $\tilde{g}_{z, \tilde{\varepsilon}}$ but provides no enhancement in computing the densities $g_{z, \varepsilon}$.

Another problem of practical interest is the reconstruction of the obstacle from measurements in a finite distance. For this situation we have already introduced a reconstruction scheme based on the knowledge of one pair of Cauchy data in Chapter 6 where we introduced the point source method. Another reconstruction algorithm that needs the knowledge of the complete Dirichlet-to-Neumann map was presented with the probe method in Chapter 7. In the following we will develop a reformulation of the singular source method that can be applied to boundary value problems. Since the method 
of singular sources needs the far field pattern of all incident plane waves for the reconstruction we also need the corresponding Cauchy data on $\partial \Omega$ and can not do with only one set of Cauchy data as for example with the PSM. This additional amount of information needed is the prize we have to pay for a reconstruction of an obstacle without the knowledge of its boundary condition.

Let us consider the following inverse acoustic boundary value problem. Given the measurement of the Cauchy data of all incident plane waves on a measurement boundary $\partial \Omega$ we want to reconstruct the sound-soft obstacle $D \subset \Omega$ in the spirit of the SSM. To this end we reformulate the approximating indicator function (8.18) with the help of the representation formula

$$
u^{\infty}(-d, \hat{x})=-\gamma_{m} \int_{\partial D}\left(u^{s}(y, \hat{x}) \frac{\partial e^{i \kappa d \cdot y}}{\partial \nu(y)}-\frac{\partial u^{s}(y, \hat{x})}{\partial \nu(y)} e^{i \kappa d \cdot y}\right) d s(y),
$$

with $d, \hat{x} \in \mathbb{S}^{m-1}$, of the far field pattern, see Theorem 2.5 in [7]. Note that the unit normal vector $\nu(y)$ is orientated into the exterior of $\bar{\Omega} \backslash D$. Inserting this representation in (8.18) and interchanging the order of integration yields

$$
\begin{aligned}
\tilde{I}(z)= & -\int_{\mathbb{S}^{m-1}} \int_{\mathbb{S}^{m-1}} \int_{\partial D} u^{s}(y, \hat{x}) \frac{\partial e^{i \kappa d \cdot y}}{\partial \nu(y)} \tilde{g}_{z, \tilde{\varepsilon}}(d) g_{z, \varepsilon}(\hat{x}) d s(y) d s(d) d s(\hat{x}) \\
& +\int_{\mathbb{S}^{m-1}} \int_{\mathbb{S}^{m-1}} \int_{\partial D} \frac{\partial u^{s}(y, \hat{x})}{\partial \nu(y)} e^{i \kappa d \cdot y} \tilde{g}_{z, \tilde{\varepsilon}}(d) g_{z, \varepsilon}(\hat{x}) d s(y) d s(d) d s(\hat{x}) \\
= & -\int_{\mathbb{S}^{m-1}} \int_{\partial D} u^{s}(y, \hat{x}) \int_{\mathbb{S}^{m-1}} \frac{\partial e^{i \kappa d \cdot y}}{\partial \nu(y)} \tilde{g}_{z, \tilde{\varepsilon}}(d) d s(d) d s(y) d s(\hat{x}) \\
& +\int_{\mathbb{S}^{m-1}} \int_{\partial D} \frac{\partial u^{s}(y, \hat{x})}{\partial \nu(y)} \int_{\mathbb{S}^{m-1}} e^{i \kappa d \cdot y} \tilde{g}_{z, \tilde{\varepsilon}}(d) d s(d) d s(y) d s(\hat{x})
\end{aligned}
$$

With the definition (5.44) of the Herglotz wave function $v_{z, \tilde{\varepsilon}}$ we can simplify this expression and obtain

$\tilde{I}(z)=-\int_{\mathbb{S}^{m-1}} \int_{\partial D}\left(u^{s}(y, \hat{x}) \frac{\partial v_{z, \tilde{\varepsilon}}}{\partial \nu}(y)-\frac{\partial u^{s}(y, \hat{x})}{\partial \nu(y)} v_{z, \tilde{\varepsilon}}(y)\right) d s(y) g_{z, \varepsilon}(\hat{x}) d s(\hat{x})$

for $z \in \mathcal{B}$. Since both functions $v_{z}$ and $\int_{\mathbb{S}^{m-1}} u^{s}(\cdot, \hat{x}) g_{z, \varepsilon}(\hat{x}) d s(\hat{x})$ solve the Helmholtz equation in $\Omega \backslash \bar{D}$ we deduce

$$
\begin{aligned}
0=\int_{\partial \bar{\Omega} \backslash D} & \left(\frac{\partial v_{z, \tilde{\varepsilon}}}{\partial \nu}(y) \int_{\mathbb{S}^{m-1}} u^{s}(y, \hat{x}) g_{z, \varepsilon}(\hat{x}) d s(\hat{x})\right. \\
& \left.-v_{z, \tilde{\varepsilon}}(y) \frac{\partial}{\partial \nu(y)} \int_{\mathbb{S}^{m-1}} u^{s}(y, \hat{x}) g_{z, \varepsilon}(\hat{x}) d s(\hat{x})\right) d s(y)
\end{aligned}
$$

and rewrite (8.24) as an integral over the boundary $\partial \Omega$ in the form 


$$
\tilde{I}(z)=\int_{\mathbb{S}^{m-1}} \int_{\partial \Omega}\left(u^{s}(y, \hat{x}) \frac{\partial v_{z, \tilde{\varepsilon}}}{\partial \nu}(y)-\frac{\partial u^{s}(y, \hat{x})}{\partial \nu(y)} v_{z, \tilde{\varepsilon}}(y)\right) d s(y) g_{z, \varepsilon}(\hat{x}) d s(\hat{x})
$$

for $z \in \mathcal{B}$. With the same argumentation we can multiply

$$
0=\int_{\partial \Omega}\left(u^{i}(y, \hat{x}) \frac{\partial v_{z, \tilde{\varepsilon}}}{\partial \nu}(y)-\frac{\partial u^{i}(y, \hat{x})}{\partial \nu(y)} v_{z, \tilde{\varepsilon}}(y)\right) d s(y)
$$

with $g_{z, \varepsilon}$, integrate over $\mathbb{S}^{m-1}$ and add this expression to the approximate indicator function. Hence we finally obtain the representation

$$
\tilde{I}(z)=\int_{\mathbb{S}^{m-1}} \int_{\partial \Omega}\left(u(y, \hat{x}) \frac{\partial v_{z, \tilde{\varepsilon}}}{\partial \nu}(y)-\frac{\partial u(y, \hat{x})}{\partial \nu(y)} v_{z, \tilde{\varepsilon}}(y)\right) d s(y) g_{z, \varepsilon}(\hat{x}) d s(\hat{x})
$$

of the approximate indicator function $\tilde{I}$ for $z \in \mathcal{B}$ in terms of the Cauchy data of the total field. For an application of the singular sources method we therefore have to measure the Cauchy data

$$
u(y, \hat{x}), \quad \frac{\partial u(y, \hat{x})}{\partial \nu(y)}, \quad y \in \partial \Omega, \hat{x} \in \mathbb{S}^{m-1}
$$

of the total field $u(\cdot, \hat{x})$ on the measurement boundary $\partial \Omega$ for all incident plane waves $u^{i}(\cdot, \hat{x})$. The modification of the indicator function $\mathcal{I}_{\text {enl }}$ of the admissibility region is straightforward and omitted here. Thus we have deduced the following reformulation of the SSM for the inverse acoustic boundary value problem.

Definition 8.4 (Singular sources method for inverse acoustic bvp). For the inverse acoustic boundary value problem the method of singular sources calculates an approximation $\tilde{I}$ to the true indicator function $I$ on subsets $\mathcal{E}_{c}$ of $\mathcal{B}$ by the following steps.

1. Choose a fixed reference configuration $\left(0, G_{0}\right)$ such that $0 \notin G_{0}$ and calculate the density $g_{0, \varepsilon} \in L^{2}\left(\mathbb{S}^{m-1}\right)$ and $\tilde{g}_{0, \tilde{\varepsilon}} \in L^{2}\left(\mathbb{S}^{m-1}\right)$ as a solution of equation (5.89) with $H$ defined by (5.45).

2. For each $z \in \mathcal{S} \subset \mathcal{B}$ choose a domain of approximation $G(z)$ of the form (5.86), i.e. the configuration $(z, G(z))$ is an orthogonal translation of the reference configuration $\left(0, G_{0}\right)$.

3. For each $z \in \mathcal{S}$ calculate the densities $g_{z, \varepsilon}, \tilde{g}_{z, \tilde{\varepsilon}} \in L^{2}\left(\mathbb{S}^{m-1}\right)$ via (5.91).

4. Calculate the indicator function $\tilde{I}(z)$ of the $S S M$ via (8.29).

5. Calculate an approximation $\mathcal{E}_{c}:=\left\{z \in \mathcal{S}:\left|\mathcal{I}_{\text {enl }}(z)\right|<c\right\}$ for the admissibility region.

6. Repeat steps 1 - 5 with a different choice of the approximation domains until the union of the domains $\mathcal{E}_{c}$ is a doubly connected domain. Extend the single indicator functions $\tilde{I}$ with a suitable weighted mean operation to this domain.

7. Choose some suitable cut-off constant $C>0$ and decide 


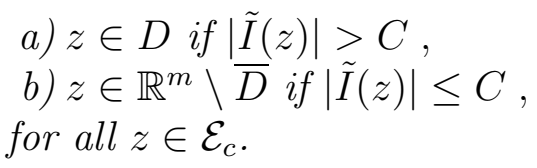

\subsection{A Comparison of Sampling Methods}

With this reformulation of the SSM to the inverse acoustic boundary value problem and the probe method of the previous chapter we now know two different reconstruction schemes in this setting. Both methods belong to the class of sampling methods and define an indicator function which blows off when the sampling point approaches the boundary of the unknown obstacle. The presented formulation of the probe method is based on the knowledge of the full Cauchy data, i.e. the Dirichlet-to-Neumann map on the measurement boundary $\partial \Omega$ while the singular sources method originally uses the far field pattern of all incident plane waves for the reconstruction. We will now compare the reconstruction schemes based on the point source approximation techniques with two well-known reconstruction schemes in inverse acoustic scattering theory, namely the linear sampling and the probe method. Since these methods use the knowledge of the far field patterns of all incident plane waves as input data we will apply the singular sources method rather than the probe method for this comparison. Moreover, in [48] Sini et al. show that the the indicator functions of the singular sources and the probe method are essentially based on the same singular term, i.e. the indicator functions fulfill the same asymptotic behaviour when the sampling point $z \in \mathcal{B}$ approaches the unknown boundary $\partial D$ of the scatterer. For this reason, we will show reconstructions of the sound-soft obstacles of Setting $1-3$ for the two-dimensional inverse scattering problem with the singular sources method, the linear sampling method and the factorization method, only. Additionally, we give reconstruction examples of these methods under the influence of random noise on the given far field data. Let us therefore recall the linear sampling and the factorization method before we present the numerical implementation together with the reconstruction examples in the next section.

The linear sampling method (LSM) was originally introduced by Colton and Kirsch in [6] and further improved by Colton, Piana and Potthast in [8]. In the following, we give a description of this method based on the techniques developed in these two papers. The linear sampling method tests if the range of the far field operator $F: L^{2}\left(\mathbb{S}^{m-1}\right) \longrightarrow L^{2}\left(\mathbb{S}^{m-1}\right)$ defined via

$$
(F g)(\hat{x}):=\int_{\mathbb{S}^{m-1}} u^{\infty}(\hat{x}, d) g(d) d s(d), \quad \hat{x} \in \mathbb{S}^{m-1}
$$

contains the function $e^{-i \kappa \hat{x} \cdot z}, \hat{x} \in \mathbb{S}^{m-1}$, which describes the far field pattern of the radiating point source $\Phi(\cdot, z)$ up to a scalar factor in both $\mathbb{R}^{2}$ and $\mathbb{R}^{3}$. To this end the LSM investigates the solvability of the far field equation 


$$
(F g)(\hat{x})=e^{-i \kappa \hat{x} \cdot z}, \quad \hat{x} \in \mathbb{S}^{m-1}
$$

for all $z \in \mathcal{B}$. For sampling points $z \in \mathcal{B} \backslash \bar{D}$ outside the scattering obstacle we can prove that the far field equation is not solvable, see [60].

Theorem 8.5. Let $D \subset \mathbb{R}^{m}$ be a sound-soft scatterer and $z \in \mathcal{B} \backslash \bar{D}$. Then the far field equation (8.32) is not solvable.

Proof. Let $z \in \mathcal{B} \backslash \bar{D}$ and suppose that $g_{z} \in L^{2}\left(\mathbb{S}^{m-1}\right)$ is a solution to (8.32). Then,

$$
w^{\infty}(\hat{x})=\int_{\mathbb{S}^{m-1}} u^{\infty}(\hat{x}, d) g_{z}(d) d s(d), \quad \hat{x} \in \mathbb{S}^{m-1}
$$

is the far field pattern of the scattered wave

$$
w^{s}(x)=\int_{\mathbb{S}^{m-1}} u^{s}(x, d) g_{z}(d) d s(d), \quad x \in \mathbb{R}^{m-1} \backslash D,
$$

which arises from the scattering of the incident Herglotz wave

$$
w^{s}(x)=\int_{\mathbb{S}^{m-1}} u^{i}(x, d) g_{z}(d) d s(d), \quad x \in \mathbb{R}^{m-1}
$$

by the obstacle $D$. Due to Rellich's lemma, the functions $w^{s}(x)$ and $\Phi(x, z)$ have to coincide for all $x \in \mathbb{R}^{m-1}(\backslash D \cup\{z\})$ up to a scalar factor. This contradicts the fact that $w^{s}$ is bounded in every neighbourhood of $z$ since $\Phi(\cdot, z)$ itself is unbounded on every neighbourhood of $z$ not including the source point.

For sampling points $z \in D$ inside the obstacle the far field equation will be solvable only in special cases, when $D$ is for example a disk with center $z$, see [6]. However, we can state the following theorem, which is cited from the original work [6] of Colton and Kirsch.

Theorem 8.6. Assume that the sound-soft obstacle $D$ is simply connected and that $-\kappa^{2}$ is not a Dirichlet eigenvalue of the Laplacian in D. Then for every $\varepsilon>0$ and $z \in D$ there exists a density $g_{z} \in L^{2}\left(\mathbb{S}^{m-1}\right)$ such that

$$
\left\|\left(F g_{z}\right)(\hat{x})-e^{-i \kappa \hat{x} \cdot z}\right\|_{L^{2}\left(\mathbb{S}^{m-1}\right)}<\varepsilon
$$

is satisfied,

$$
\lim _{z \rightarrow \partial D}\left\|g_{z}\right\|_{L^{2}\left(\mathbb{S}^{m-1}\right)}=\infty
$$

and, if $v_{z}$ is the Herglotz wave function

$$
v_{z}(x)=\int_{\mathbb{S}^{m-1}} e^{i \kappa x \cdot d} g_{z}(d) d s(d)
$$

with kernel $g_{z}$,

$$
\lim _{z \rightarrow \partial D} \max _{x \in \partial D}\left(\left|v_{z}(x)\right|+\left|\nabla v_{z}(x)\right|\right)=\infty
$$

holds. 
For the solution of the ill-posed integral equation (8.32) of the first kind we use Morozov's discrepancy principle for a Tikhonov regularization scheme as presented in [8]. We give a brief introduction to this method in the following. We consider noisy far field data $u_{\delta}^{\infty}(\hat{x}, d)$ which is given for all $\hat{x}, d \in \mathbb{S}^{m-1}$. Hence we do not know the exact far field operator $F$ but we have access to the noisy far field operator $F_{\delta}$ defined by the noisy kernel $u_{\delta}^{\infty}(\hat{x}, d)$ such that $\left\|F-F_{\delta}\right\| \leq \delta$ holds. On the other hand, the right hand side $r_{z}=e^{-i \kappa \hat{x} \cdot z}$ is known exactly for all $z \in \mathcal{B}$ and $\hat{x} \in \mathbb{S}^{m-1}$. Thus a Tikhonov regularization method with regularization parameter $\alpha>0$ is equivalent to solving the minimum problem

$$
\left\|F_{\delta} g_{\alpha}-r_{z}\right\|_{L^{2}\left(\mathbb{S}^{m-1}\right)}^{2}+\alpha\left\|g_{\alpha}\right\|_{L^{2}\left(\mathbb{S}^{m-1}\right)}^{2}=\min .
$$

Now, the general discrepancy principle suggests to choose the regularization parameter $\alpha$ to be either the zero of the monotonically increasing generalized discrepancy function

$$
\mu(\alpha)=\left\|F_{\delta} g_{\alpha}-r_{z}\right\|_{L^{2}\left(\mathbb{S}^{m-1}\right)}^{2}-\delta^{2}\left\|g_{\alpha}\right\|_{L^{2}\left(\mathbb{S}^{m-1}\right)}^{2}, \quad \alpha>0
$$

if there exists an $\alpha>0$ with $\mu(\alpha)=0$, or to be the limit of $\mu$ for $\alpha \rightarrow 0$. Finally, we obtain for every $z \in \mathcal{B}$ the value of the indicator function

$$
I_{\mathrm{LSM}}(z)=\left(\left\|g_{\alpha}(z)\right\|_{L^{2}\left(\mathbb{S}^{m-1}\right)}\right)^{-1},
$$

where the regularization parameter $\alpha=\alpha(z)$ is chosen with the generalized discrepancy principle.

In 1998, two years after Colton and Kirsch introduced their linear sampling method to the scientific community, Kirsch presented a precise criterion for the sampling point $z$ to belong to the scatterer $D$ by investigating a factorization of the far field operator $F$ in his outstanding paper on a characterization of the shape of a scattering obstacle using the spectral data of the far field operator, see [32]. He investigates the properties of the operator $\left(F^{*} F\right)^{1 / 4}$ and proves that its range coincides with the range of the operator $G$ mapping functions on the boundary $\partial D$ of an obstacle $D$ into the far field pattern of the corresponding exterior boundary value problem. We summarize the main result of the factorization method in the next theorem which we cite from the original work [32] of Kirsch.

Theorem 8.7. Assume $-\kappa^{2}$ is not a Dirichlet eigenvalue of the Laplacian in $D$. Then we have the following characterization of $D$ :

$$
D=\left\{z \in \mathbb{R}^{m}: \sum_{j=1}^{\infty} \frac{\left|\varrho_{j}^{(z)}\right|^{2}}{\sigma_{j}}<\infty\right\}=\left\{z \in \mathbb{R}^{m}: r_{z} \in \mathcal{R}\left(\left(F^{*} F\right)^{1 / 4}\right)\right\}
$$

where $\left\{\sigma_{j}, \psi_{j}, \tilde{\psi}_{j}\right\}$ is a singular system of $F$, and $\varrho_{j}^{(z)}$ are the expansion coefficients of $r_{z}(\hat{x})=e^{-i \kappa \hat{x} \cdot z}, \hat{x} \in \mathbb{S}^{m-1}$, with respect to $\left\{\tilde{\psi}_{j}: j \in \mathbb{N}\right\}$, i.e. $\varrho_{j}^{(z)}=\left\langle r_{z}, \tilde{\psi}_{j}\right\rangle_{L^{2}}$ 
For the regularization of the equation

$$
\left(F^{*} F\right)^{1 / 4} g=r_{z}
$$

we again use the generalized discrepancy principle by solving the minimizing problem

$$
\left\|\left(F_{\delta}^{*} F_{\delta}\right)^{1 / 4} g_{\alpha}-r_{z}\right\|_{L^{2}\left(\mathbb{S}^{m-1}\right)}^{2}+\alpha\left\|g_{\alpha}\right\|_{L^{2}\left(\mathbb{S}^{m-1}\right)}^{2}=\min
$$

and choosing the regularization parameter analogously as explained for the linear sampling method. Then the indicator function of the factorization method is given by

$$
I_{\mathrm{FM}}(z)=\left(\left\|g_{\alpha}(z)\right\|_{L^{2}\left(\mathbb{S}^{m-1}\right)}\right)^{-1}, \quad z \in \mathcal{B},
$$

where the density $g_{\alpha} \in L^{2}\left(\mathbb{S}^{m-1}\right)$ is the unique solution of (8.45) with the regularization parameter $\alpha=\alpha(z)$ chosen by the generalized discrepancy principle.

For the numerical implementation of both the linear sampling and the factorization method we first calculate a singular system $\left\{\sigma_{j}, \psi_{j}, \tilde{\psi}_{j}\right\}$ of $F$. We refer to [36] for a summary of the singular value decomposition and the following reformulation of the Tikhonov regularization scheme in terms of the singular system of the far field operator. Using the representations

$$
F g=\sum_{j=1}^{\infty} \sigma_{j}\left\langle g, \psi_{j}\right\rangle_{L^{2}} \tilde{\psi}_{j}
$$

and

$$
\left(F^{*} F\right)^{1 / 4} g=\sum_{j=1}^{\infty} \sqrt{\sigma_{j}}\left\langle g, \psi_{j}\right\rangle_{L^{2}} \tilde{\psi}_{j}
$$

we observe that the Tikhonov solution is given by

$$
g_{\alpha}=\sum_{j=1}^{\infty} \frac{\sigma_{j}}{\alpha+\sigma_{j}^{2}} \varrho_{j}^{(z)} \psi_{j}
$$

and

$$
g_{\alpha}=\sum_{j=1}^{\infty} \frac{\sqrt{\sigma_{j}}}{\alpha+\sigma_{j}} \varrho_{j}^{(z)} \psi_{j}
$$

with the norm

$$
\left\|g_{\alpha}\right\|_{L^{2}\left(\mathbb{S}^{m-1}\right)}^{2}=\sum_{j=1}^{\infty} \frac{\sigma_{j}^{2}}{\left(\alpha+\sigma_{j}^{2}\right)^{2}}\left|\varrho_{j}^{(z)}\right|^{2}
$$

and

$$
\left\|g_{\alpha}\right\|_{L^{2}\left(\mathbb{S}^{m-1}\right)}^{2}=\sum_{j=1}^{\infty} \frac{\sigma_{j}}{\left(\alpha+\sigma_{j}\right)^{2}}\left|\varrho_{j}^{(z)}\right|^{2}
$$


respectively. For the determination of the regularization parameter we use the expansions

$$
\mu(\alpha)=\sum_{j=1}^{\infty} \frac{\alpha^{2}-\delta^{2} \sigma_{j}^{2}}{\left(\alpha+\sigma_{j}^{2}\right)^{2}}\left|\varrho_{j}^{(z)}\right|^{2}
$$

and

$$
\mu(\alpha)=\left.\sum_{j=1}^{\infty} \frac{\alpha^{2}-\delta^{2} \sigma_{j}}{\left(\alpha+\sigma_{j}\right)^{2}} \varrho_{j}^{(z)}\right|^{2}
$$

of the discrepancy functions. Then we solve $\mu(\alpha)=0$ for $\alpha>0$ with a simple numeric scheme such as the secant method.

In a discretized setting we assume the knowledge of the matrix $A=$ $\left(u^{\infty}\left(d_{j}, d_{l}\right)\right) \in \mathbb{C}^{M \times M}$ of the far field pattern at equidistant directions $d_{j}=2 \pi j / M$ for $j=1, \ldots, M$. With the singular value decomposition of the matrix $A$ we obtain two normal matrices $U$ and $V$ such that $A=U \Lambda V^{*}$ with the diagonal matrix $\Lambda$ containing the singular values of $A$. Now the discrete singular system is given by the diagonal of the matrix $\Lambda$ and the columns of the matrices $V$ and $U$, respectively. Therefore, for each $z \in \mathcal{S}$ we compute the expansion coefficients $\varrho_{l}^{(z)}$ for $l=1, \ldots, M$ with the matrix-vector multiplication

$$
\varrho_{l}^{(z)}=\sum_{j=1}^{M} \bar{U}_{j, l} e^{-i \kappa z \cdot d_{j}}, \quad l=1, \ldots, M
$$

Then we apply the method of secants to determine the unique zero $\alpha(z)$ of the discrete discrepancy function, where we replace equations (8.53) and (8.54) by their corresponding finite sums and insert the known or estimated error level $\delta$ of the noise on the far field pattern. Finally, we evaluate the indicator functions (8.42) and (8.46) for this optimal $\alpha(z)$ with the finite sums corresponding to (8.51) and (8.52), respectively. Motivated by a remark in [8], we also illustrate the behaviour of the regularization parameter $\alpha(z)$ chosen by the generalized discrepancy principle.

The numerical implementation of the singular sources method is a straightforward modification of the algorithms presented in Chapter 6 and 7. For this reason we will omit the details here but we will come back to the numerical implementation of the singular sources method in the next section when we present reconstructions for the inverse scattering problem in $\mathbb{R}^{3}$. Now, we will compare the LSM, FM and SSM in the two-dimensional inverse scattering problems with the obstacles of Setting $1-3$. The reconstructions with the SSM are obtained with the adapted approximation domains illustrated in Figure 6.9 and Figure 6.12. For the weighted indicator function we choose the same parameters $\tau=40$ and $r=0.3$ as in the previous examples with the PSM and the probe method. To investigate the stability of these reconstruction schemes we add random noise $u_{j, l}^{\delta} \in \mathbb{C}^{M \times M}$ of level $\delta>0$ on the far field pattern $u_{j, l}^{\infty}$ such that the inequality 


$$
\frac{\delta}{2}\left(\sum_{j, l=1}^{M}\left|u_{j, l}^{\infty}\right|^{2}\right)^{1 / 2} \leq\left(\sum_{j, l=1}^{M}\left|u_{j, l}^{\delta}\right|^{2}\right)^{1 / 2} \leq \delta\left(\sum_{j, l=1}^{M}\left|u_{j, l}^{\infty}\right|^{2}\right)^{1 / 2}
$$

is satisfied. The noisy far field pattern $u_{j, l}^{\infty, \delta}=u_{j, l}^{\infty}+u_{j, l}^{\delta}$ is illustrated exemplarily for the ellipse in Figure 8.1 for the error levels $\delta=0.01,0.05,0.1$ and $\delta=0.2$. We remark that due to the error in the numerical computa-
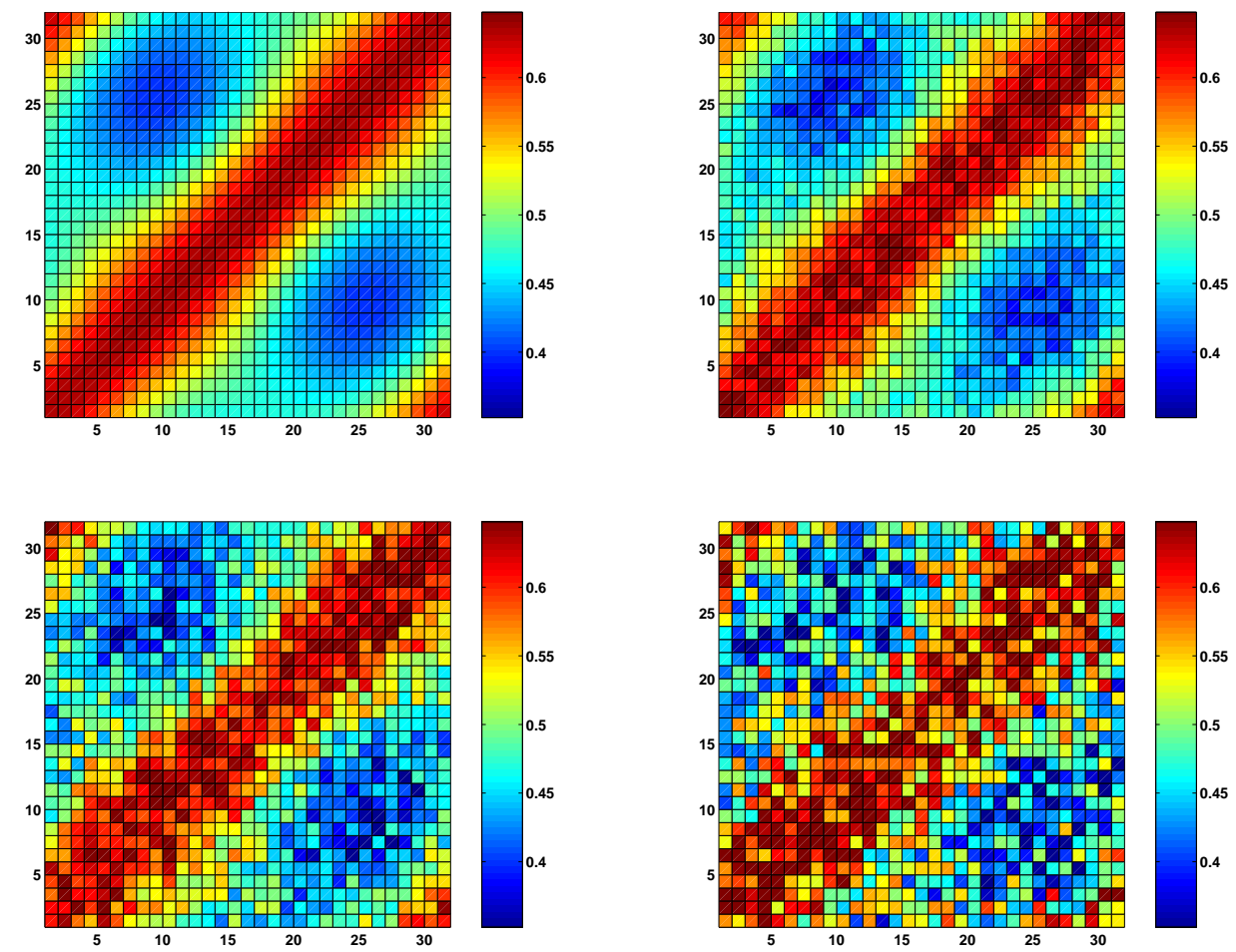

Fig. 8.1. The modulus $\left|u_{j, l}^{\infty, \delta}\right|, j, l=1, \ldots, 32$ of the noisy far field pattern for the ellipse of Setting 1 is illustrated. The figures show a noise level of $\delta=0.01$ (upper left), $\delta=0.05$ (upper right), $\delta=0.1$ (lower left) and $\delta=0.2$ (lower right). The wave number in this example was $\kappa=2.0$.

tion of the far field pattern, we obtain an intrinsic error on the far field pattern. Hence, for the noise-free far field pattern we assume an error level of $\delta=0.01$ for the determination of the regularization parameter with the generalized discrepancy principle. With $M=32$ incident and observed directions we first reconstruct the ellipse of Setting 1 with the LSM, FM and SSM. The reconstructions from the unperturbated far field pattern are illustrated in Figure 8.2. Additionally, we consider a reconstruction using the values of the regularization parameter $\alpha=\alpha(z)$ as an indicator function for both the LSM and the FM as suggested in [8]. For the SSM we plot the function $\mathcal{I}_{\text {enl }}$ as an additional indicator for the unknown obstacle. These functions together with a boundary reconstruction are illustrated in Figure 

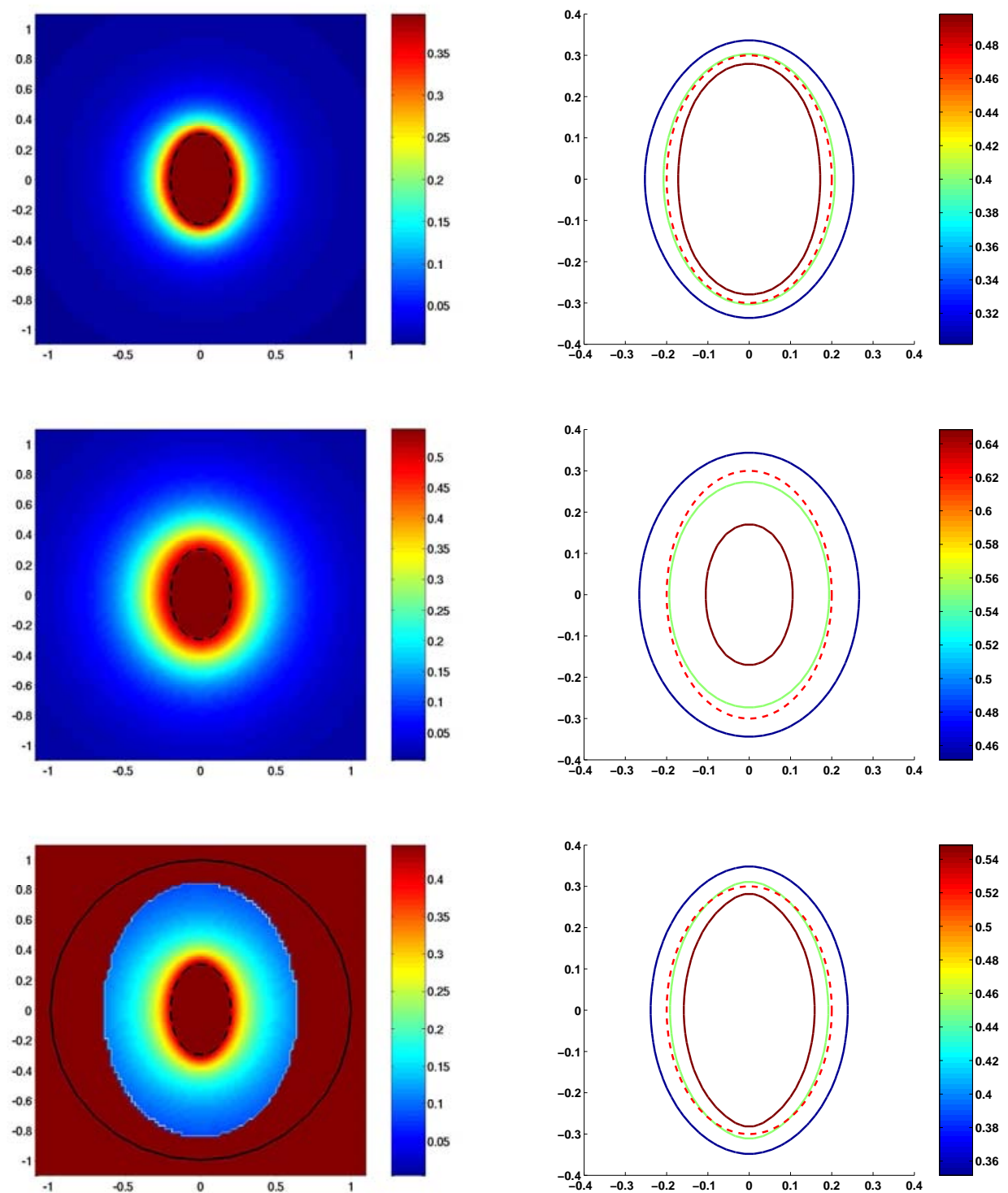

Fig. 8.2. The reconstruction of the ellipse without noise. The first row shows the indicator function of the LSM together with the boundary reconstruction. The middle row illustrates the indicator function of the factorization method while the last row shows the result for the SSM. The regularization parameter was chosen with the generalized discrepancy principle with $\delta=0.01$ for the SSM and FM. For the regularization of the SSM we chose the value $\alpha=10^{-8}$. The obstacle is indicated with a dashed line. The wave number in this example was $\kappa=2.0$. 

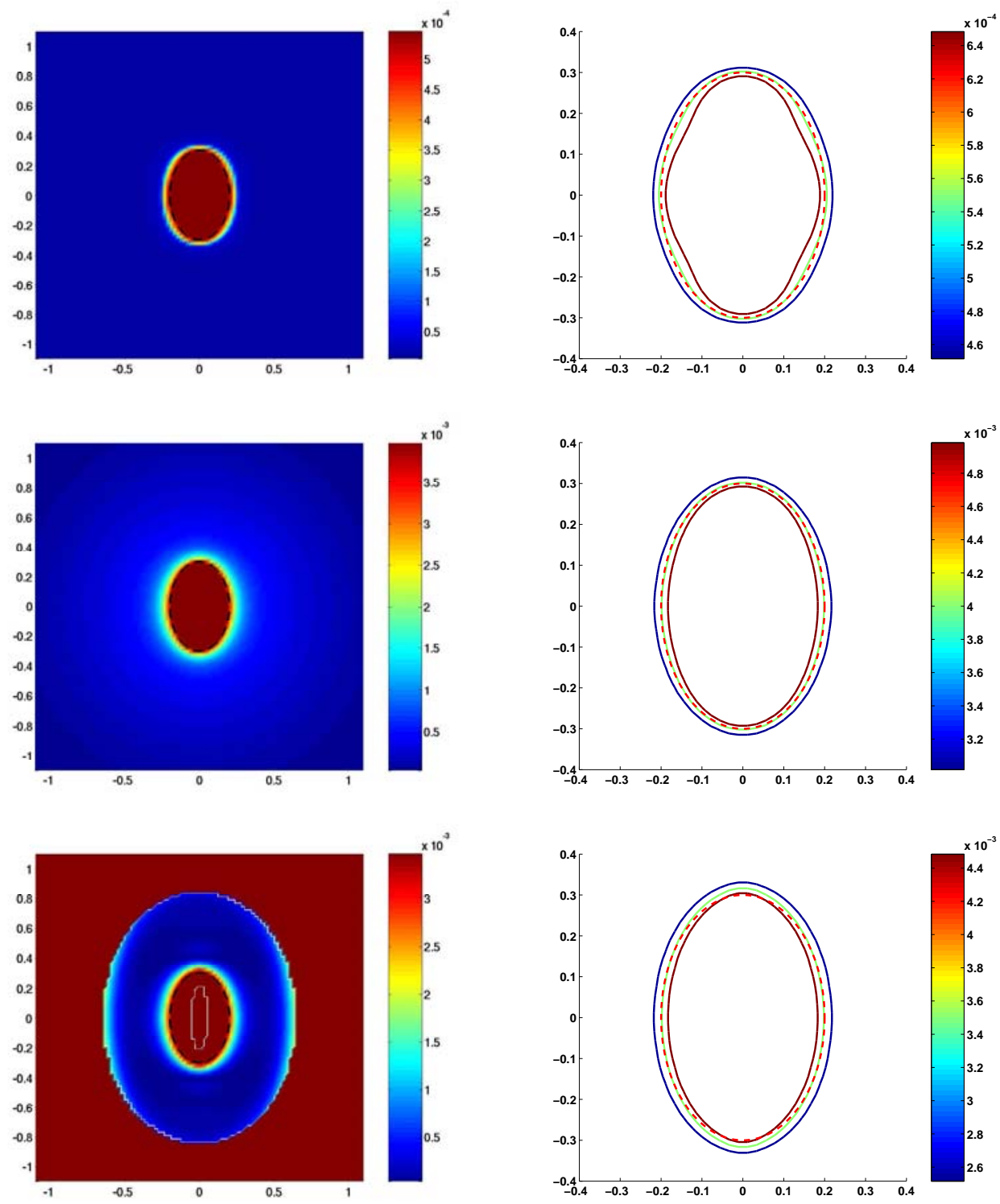

Fig. 8.3. The reconstruction of the ellipse without noise. The first row shows the map $z \mapsto \alpha(z)$ for the LSM together with the boundary reconstruction while the second row illustrates the analog results for the FM. The last row illustrates the indicator function $\mathcal{I}_{\text {enl }}$ which is the difference of two reconstructions with the SSM obtained with different regularization parameters $\alpha_{1}=10^{-8}$ and $\alpha_{2}=2 \cdot 10^{-8}$. The obstacle is indicated with a dashed line. The wave number in this example was $\kappa=2.0$.

8.3. For all methods, these heuristic indicator functions provide a sharper edge of the obstacle's boundary. In particular, the factorization method can be improved significantly by considering the mapping $z \mapsto \alpha(z)$.

For each of these methods and every error level $\delta$ we choose a suitable level set of the indicator function that fits the boundary of the ellipse. This is done by trial and error. Then we store these values of the boundary level sets and use them for the reconstruction of the other obstacles. In this 

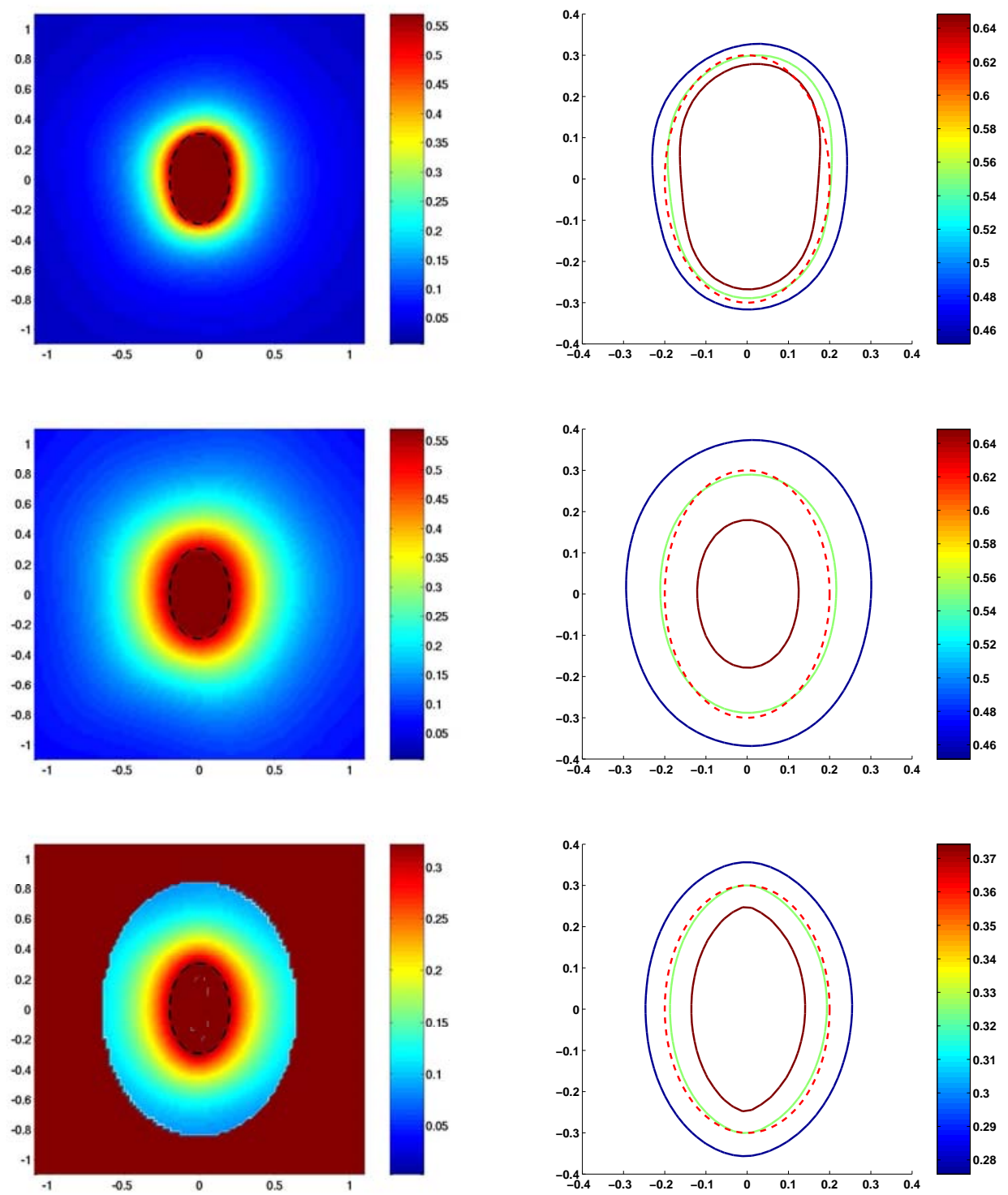

Fig. 8.4. The reconstruction of the ellipse with $5 \%$ noise. The first row shows the indicator function of the LSM together with the boundary reconstruction. The middle row illustrates the indicator function of the factorization method while the last row shows the result for the SSM. The regularization parameter was chosen with the generalized discrepancy principle with $\delta=0.05$ for the SSM and FM. For the regularization of the SSM we chose the value $\alpha=10^{-2}$. The obstacle is indicated with a dashed line. The wave number in this example was $\kappa=2.0$.

way, the reconstruction of the ellipse can be seen as a calibration of the presented sampling methods. Figure $8.2-8.9$ illustrate this calibration procedure. Figure 8.5 proves that the additional indicator for the LSM yields worse reconstructions for noisy data of $5 \%$ magnitude. The reconstructions with this indicator gets even worse for an increasing error level and is therefore omitted in the following. We will now plot the same level sets in the case of the obstacles of Setting 2 and 3. The reconstructions are illustrated 

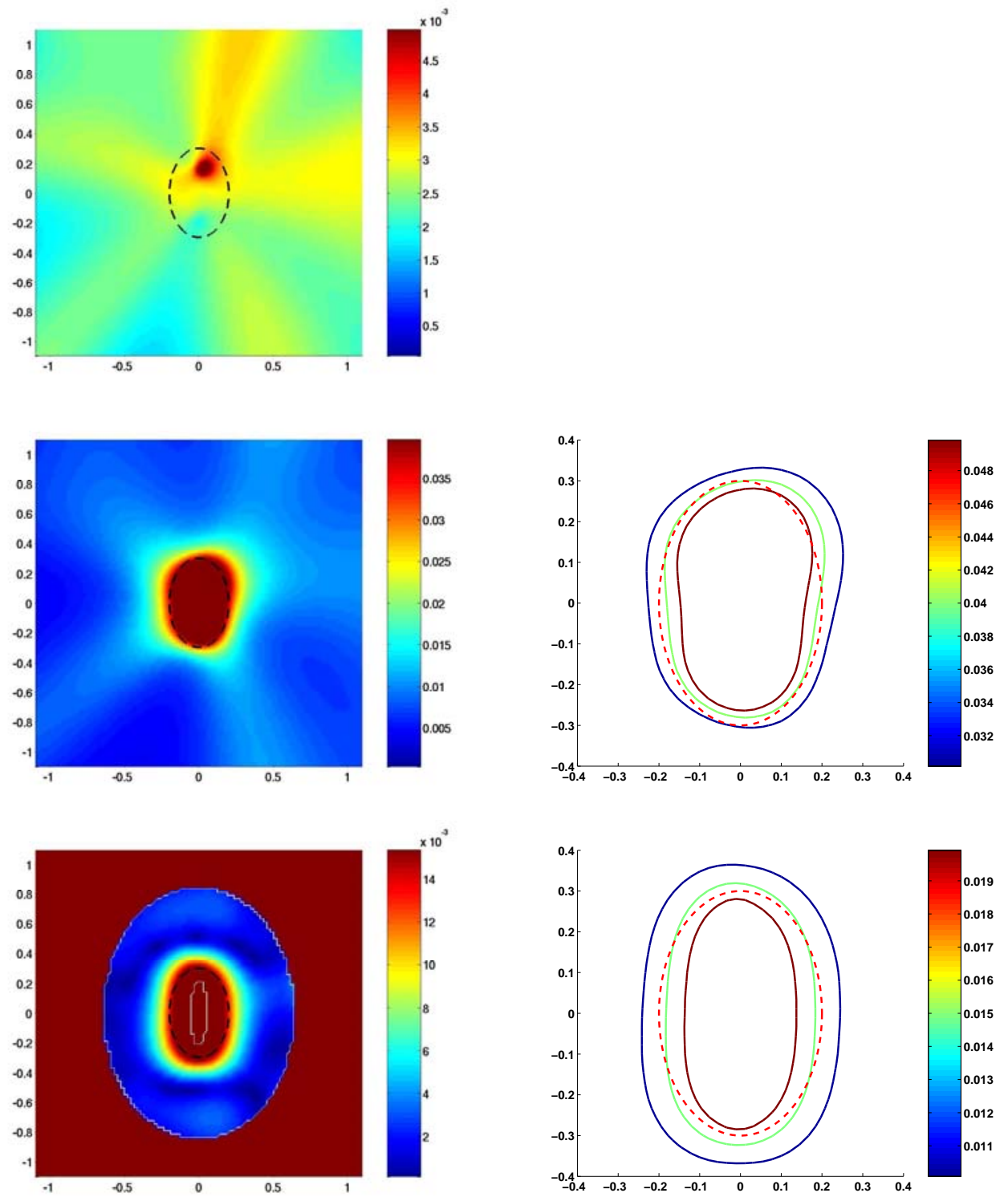

Fig. 8.5. The reconstruction of the ellipse with $5 \%$ noise. The first row shows the map $z \mapsto \alpha(z)$ for the LSM while the second row shows the same result for the FM and, additionally illustrates a boundary reconstruction. The last row illustrates the indicator function $\mathcal{I}_{\text {enl }}$ which is the difference of two reconstructions with the SSM obtained with different regularization parameters $\alpha_{1}=10^{-2}$ and $\alpha_{2}=2 \cdot 10^{-2}$. The obstacle is indicated with a dashed line. The wave number in this example was $\kappa=2.0$. 

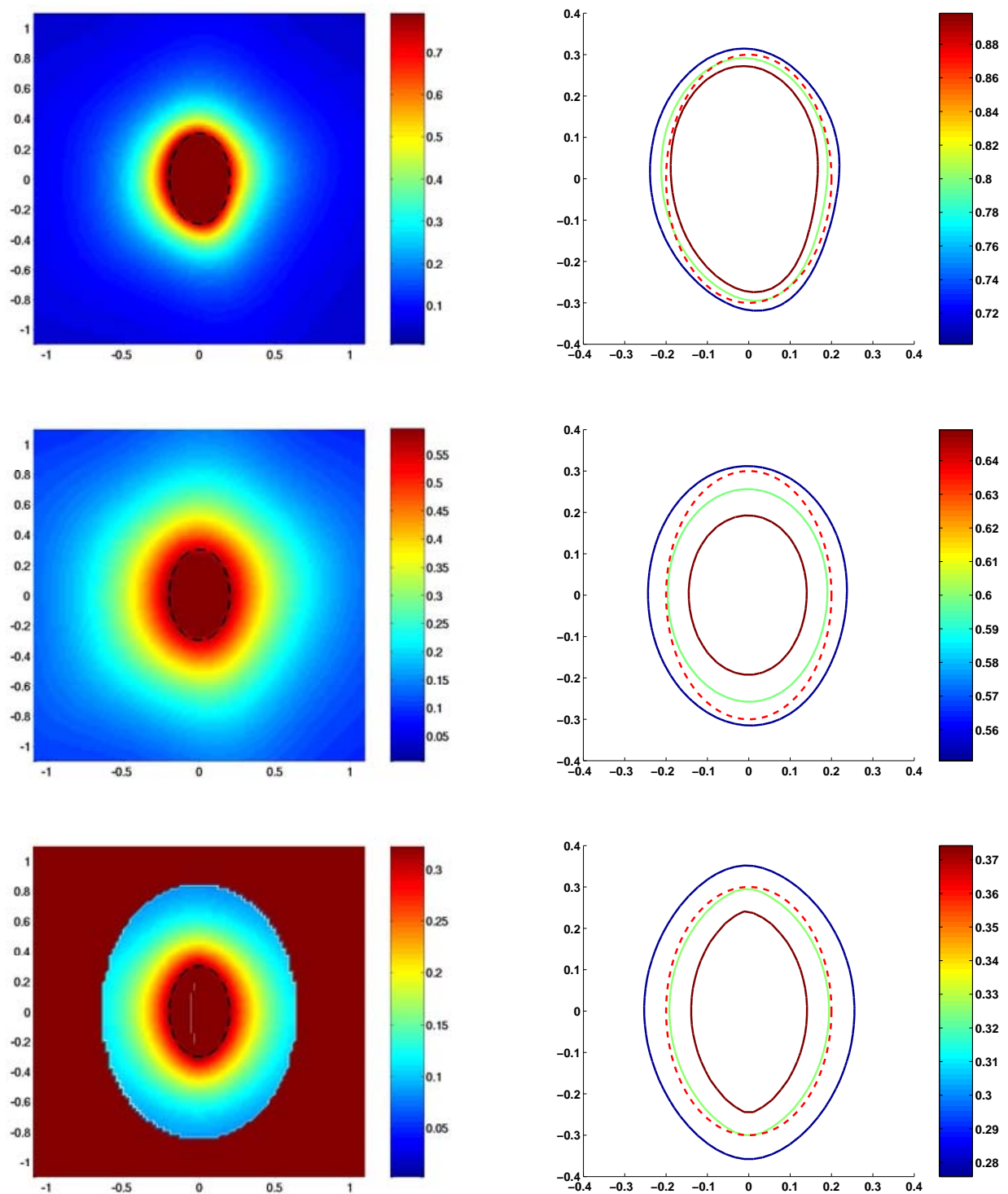

Fig. 8.6. The reconstruction of the ellipse with $10 \%$ noise. The first row shows the indicator function of the LSM together with the boundary reconstruction. The middle row illustrates the indicator function of the factorization method while the last row shows the result for the SSM. The regularization parameter was chosen with the generalized discrepancy principle with $\delta=0.1$ for the SSM and FM. For the regularization of the SSM we chose the value $\alpha=10^{-2}$. The obstacle is indicated with a dashed line. The wave number in this example was $\kappa=2.0$. 

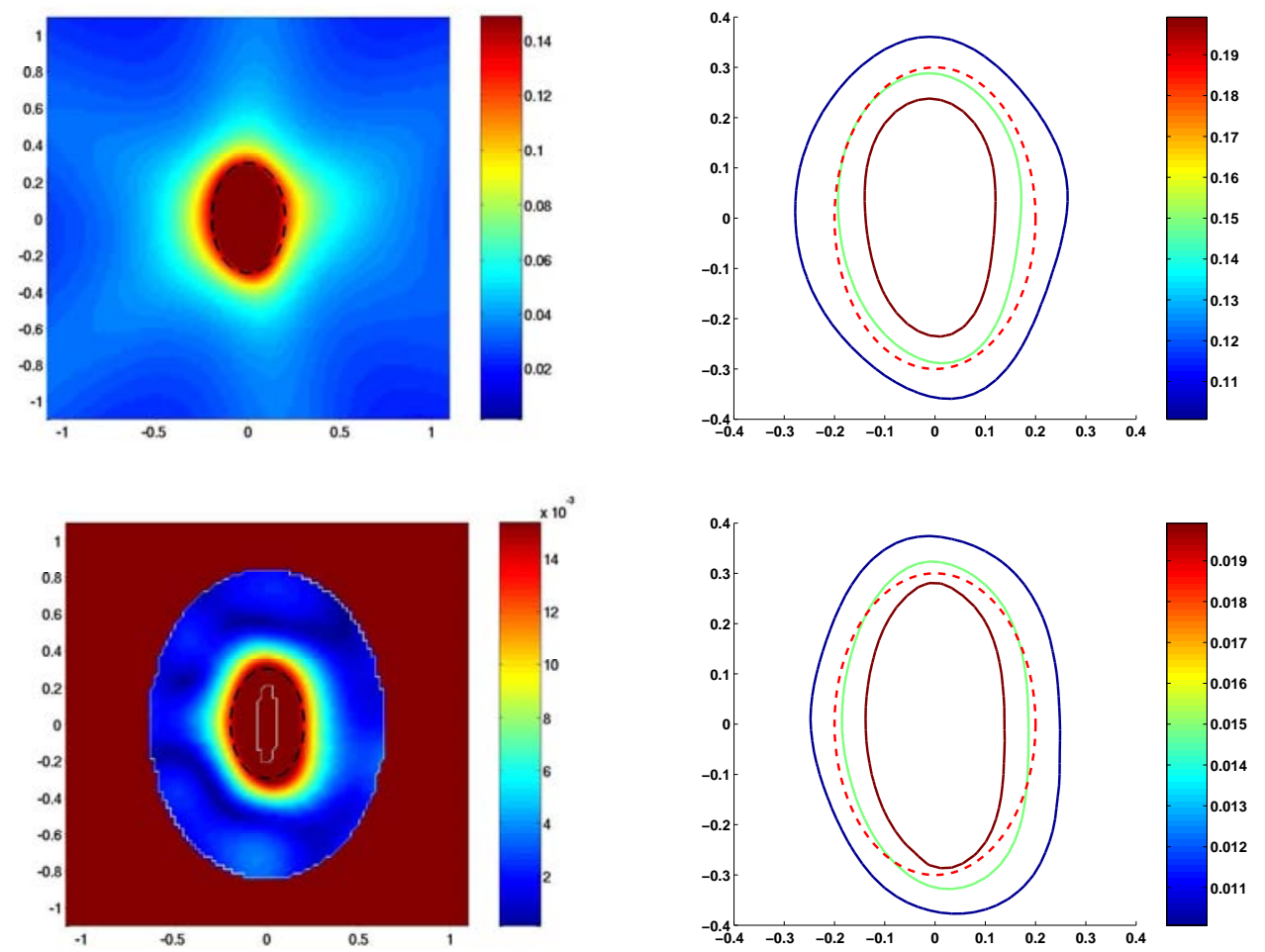

Fig. 8.7. The reconstruction of the ellipse with $10 \%$ noise. The first row shows the map $z \mapsto \alpha(z)$ for the FM together with a boundary reconstruction. The second row illustrates the indicator function $\mathcal{I}_{\text {enl }}$ which is the difference of two reconstructions with the SSM obtained with different regularization parameters $\alpha_{1}=10^{-2}$ and $\alpha_{2}=2 \cdot 10^{-2}$. The obstacle is indicated with a dashed line. The wave number in this example was $\kappa=2.0$.

in Figure 8.10 - 8.17. Let us consider the reconstruction of the kite first. Without noise, the SSM, LSM and the indicator function $\mathcal{I}_{\text {enl }}$ reconstruct the kite better than the factorization method as demonstrated with Figure 8.10. The parameter choice rule $z \mapsto \alpha(z)$ provides better results for the reconstruction of the kite than the factorization method itself, in particular in case of medium noise of level $\delta=0.05$, see Figure 8.11. In this regime, the LSM also yields a comparable reconstruction wheras the SSM starts to ignore the non-convexity of the kite. Increasing the error level further to a noise of $10-20 \%$, see Figure 8.12 and 8.13 , the situation changes again and we observe that the SSM yields the most stable reconstruction in this case. For the obstacle of Setting 3, the situation is similar, compare with Figure $8.14-8.17$.

The numerical study of the presented sampling methods shows that the SSM provides reconstructions of the investigated obstacles with a competitive quality. In particular in the case of exact far field data the reconstructions with the SSM yield the most accurate results. This is a consequence of the two-step strategy and the special choice of the adapted approximation domains that have been used in the second step of the algorithm. With 

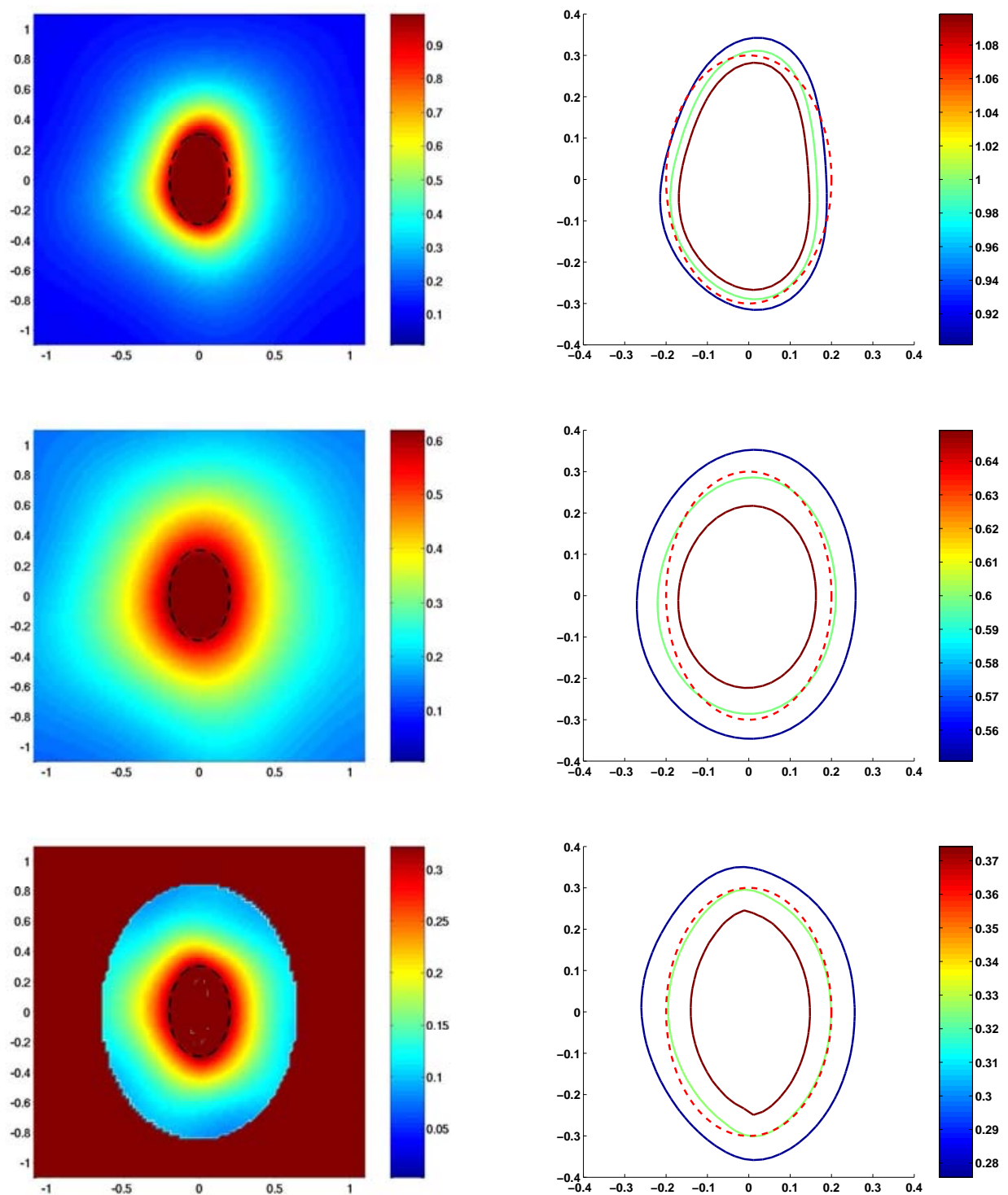

Fig. 8.8. The reconstruction of the ellipse with $20 \%$ noise. The first row shows the indicator function of the LSM together with the boundary reconstruction. The middle row illustrates the indicator function of the factorization method while the last row shows the result for the SSM. The regularization parameter was chosen with the generalized discrepancy principle with $\delta=0.2$ for the SSM and FM. For the regularization of the SSM we chose the value $\alpha=10^{-2}$. The obstacle is indicated with a dashed line. The wave number in this example was $\kappa=2.0$. 

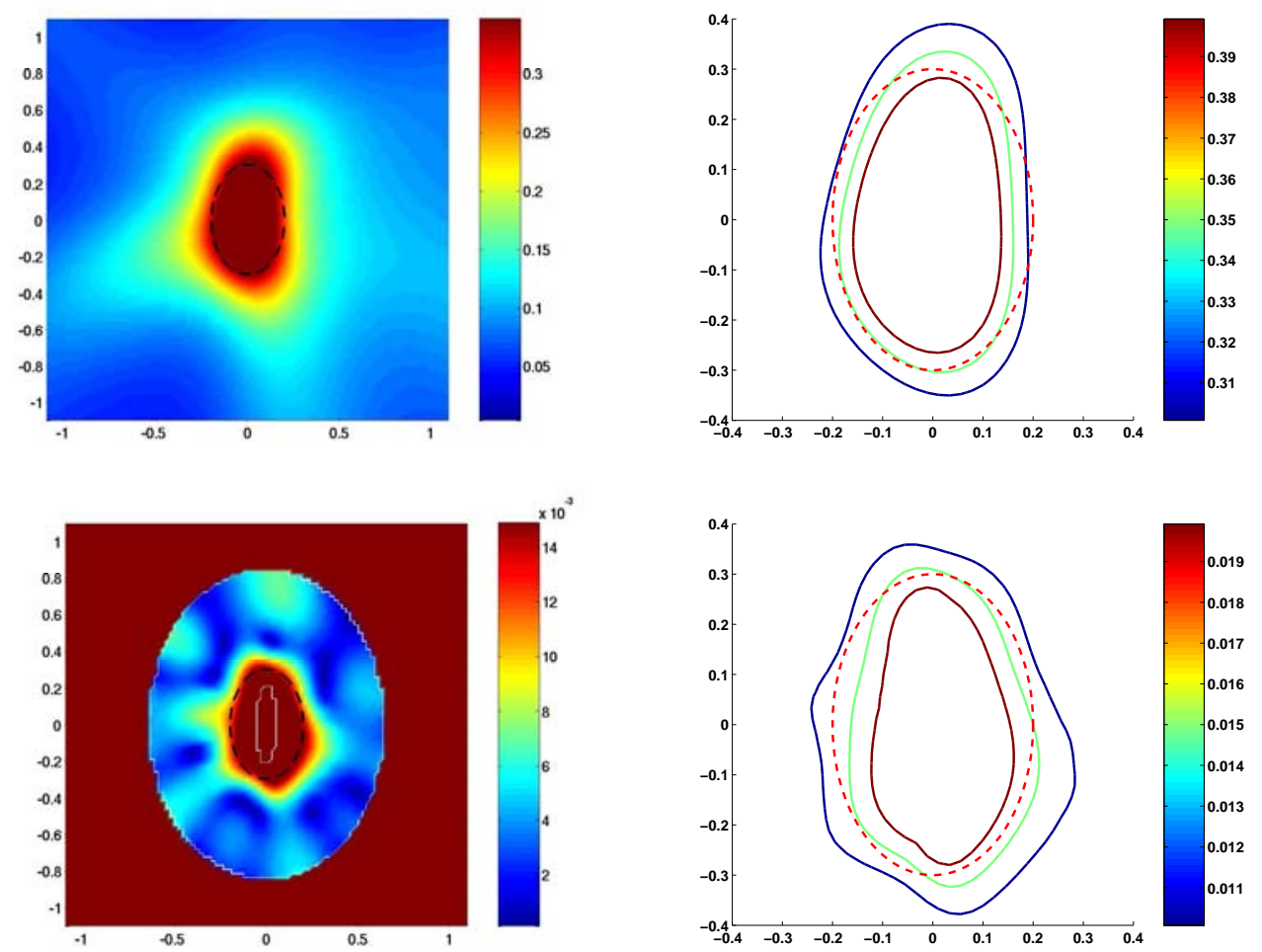

Fig. 8.9. The reconstruction of the ellipse with $20 \%$ noise. The first row shows the map $z \mapsto \alpha(z)$ for the FM together with a boundary reconstruction. The second row illustrates the indicator function $\mathcal{I}_{\text {enl }}$ which is the difference of two reconstructions with the SSM obtained with different regularization parameters $\alpha_{1}=10^{-2}$ and $\alpha_{2}=2 \cdot 10^{-2}$. The obstacle is indicated with a dashed line. The wave number in this example was $\kappa=2.0$.

the adaption of the approximation domain we put additional information, which has been governed in the first step of the algorithm, into the reconstruction algorithm of the SSM. If the error on the data increases and the far field pattern is perturbed with medium noise of up to $10 \%$, this advantage of the SSM vanishes and the LSM yields better results. Furthermore, the reconstructions with the FM are of less quality than the reconstructions obtained with the LSM, but the FM is more stable with respect to noise on the data. Using the regularization parameter $\alpha=\alpha(z)$ of the FM as indicator function yields reconstructions that are similar to the reconstructions with the LSM and provide an improvement of the reconstruction with the FM. For very high noise on the far field data the SSM proves to be the most stable reconstruction scheme with the best approximation to the scatterer. This is once again a consequence of the implicit information that is used by the choice of the approximation domains. Hence, the choice of the approximation domains can bee seen as an intrinsic regularization of the SSM. 

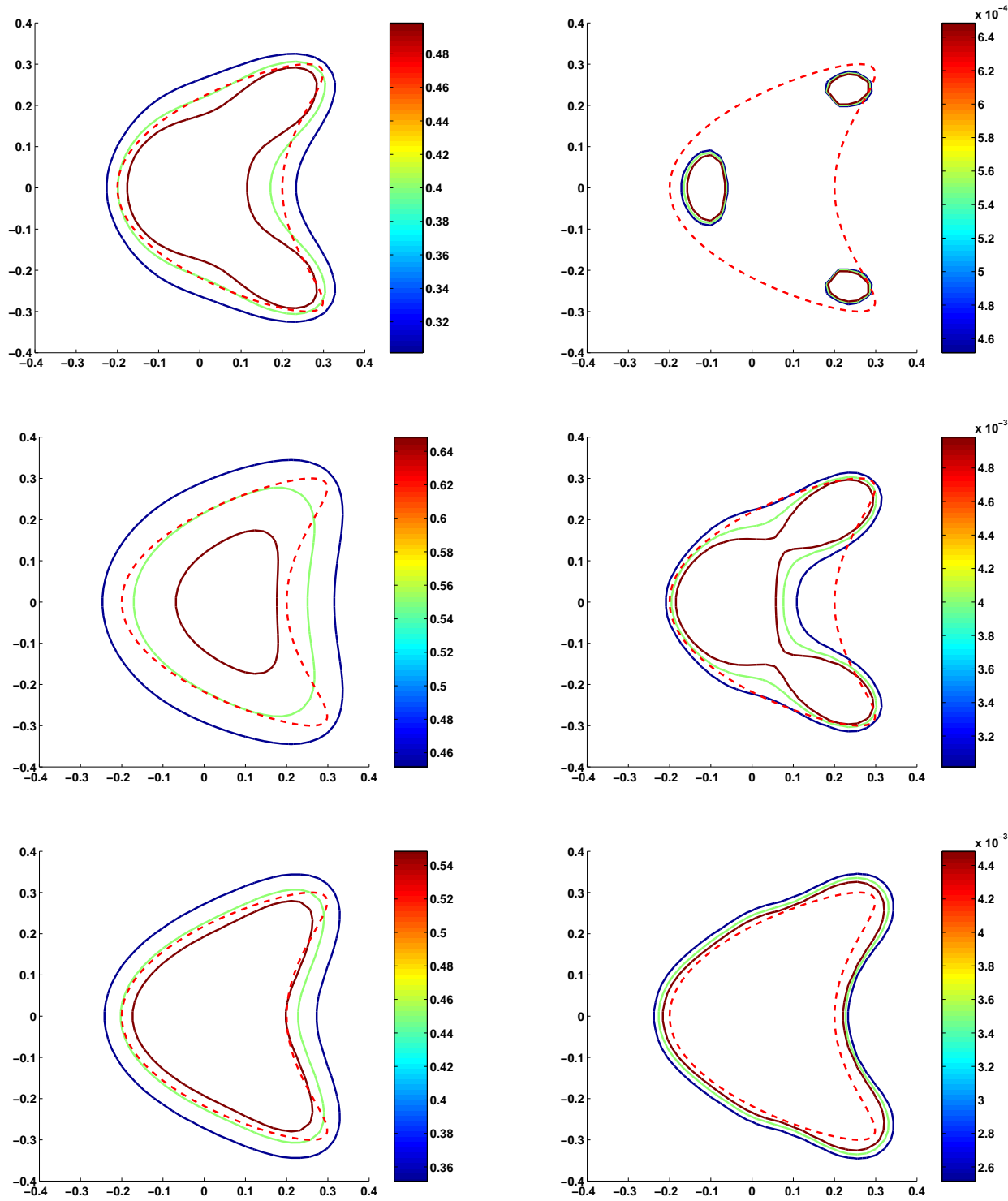

Fig. 8.10. Boundary reconstructions of the kite without noise. The first row shows the reconstruction with the indicator function of the LSM (left) and with the map $z \mapsto \alpha(z)$ as indicator function (right). The middle row illustrates the analogous reconstructions with the FFM while the last row shows the boundary reconstruction with the indicator function of the SSM (left) and the function $\mathcal{I}_{\text {enl }}$ (right). The regularization parameter was chosen with the generalized discrepancy principle with $\delta=0.01$ for the SSM and FM. For the regularization of the SSM we chose the value $\alpha=10^{-8}$. The obstacle is indicated with a dashed line. The wave number in this example was $\kappa=2.0$. 

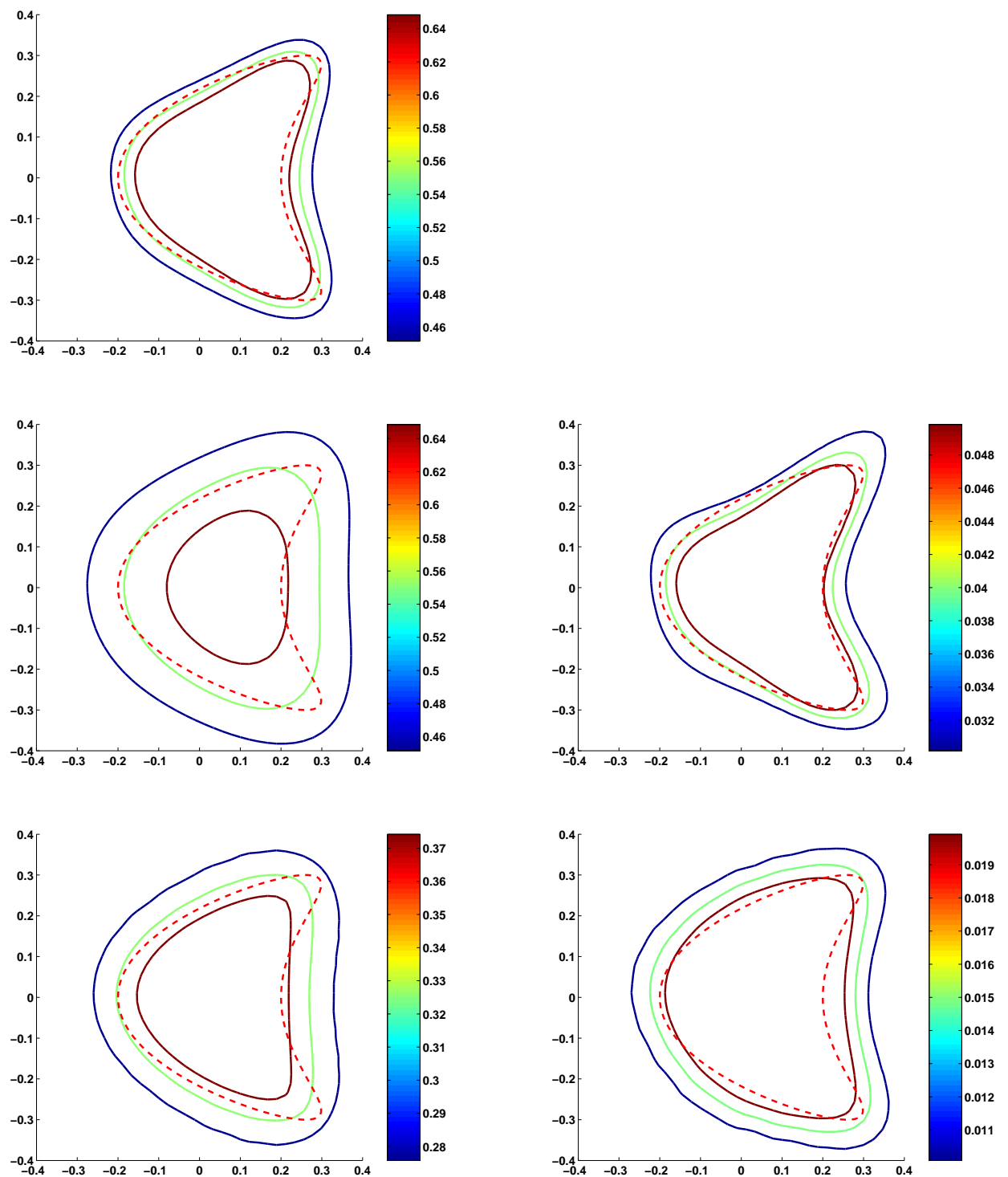

Fig. 8.11. Boundary reconstructions of the kite $5 \%$ noise. The first row shows the reconstruction with the indicator function of the LSM (left) The middle row illustrates the analogous reconstructions with the FFM (left) but additionally the reconstruction based on the map $z \mapsto \alpha(z)$. The last row shows the boundary reconstruction with the indicator function of the SSM (left) and the function $\mathcal{I}_{\text {enl }}$ (right). The regularization parameter was chosen with the generalized discrepancy principle with $\delta=0.05$ for the SSM and FM. For the regularization of the SSM we chose the value $\alpha=10^{-2}$. The obstacle is indicated with a dashed line. The wave number in this example was $\kappa=2.0$. 

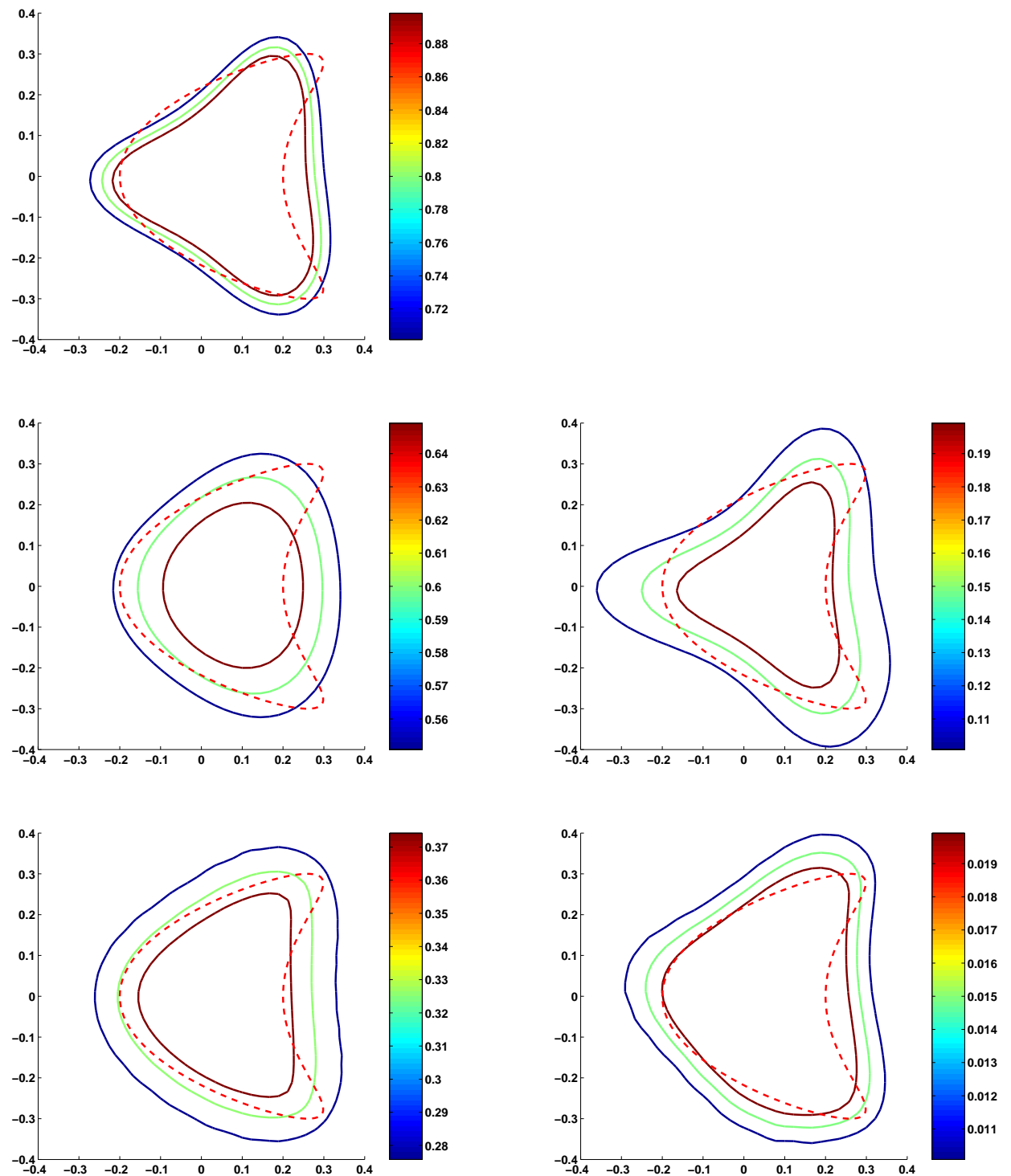

Fig. 8.12. Boundary reconstructions of the kite $10 \%$ noise. The first row shows the reconstruction with the indicator function of the LSM (left) The middle row illustrates the analogous reconstructions with the FFM (left) but additionally the reconstruction based on the map $z \mapsto \alpha(z)$. The last row shows the boundary reconstruction with the indicator function of the SSM (left) and the function $\mathcal{I}_{\text {enl }}$ (right). The regularization parameter was chosen with the generalized discrepancy principle with $\delta=0.1$ for the SSM and FM. For the regularization of the SSM we chose the value $\alpha=10^{-2}$. The obstacle is indicated with a dashed line. The wave number in this example was $\kappa=2.0$. 

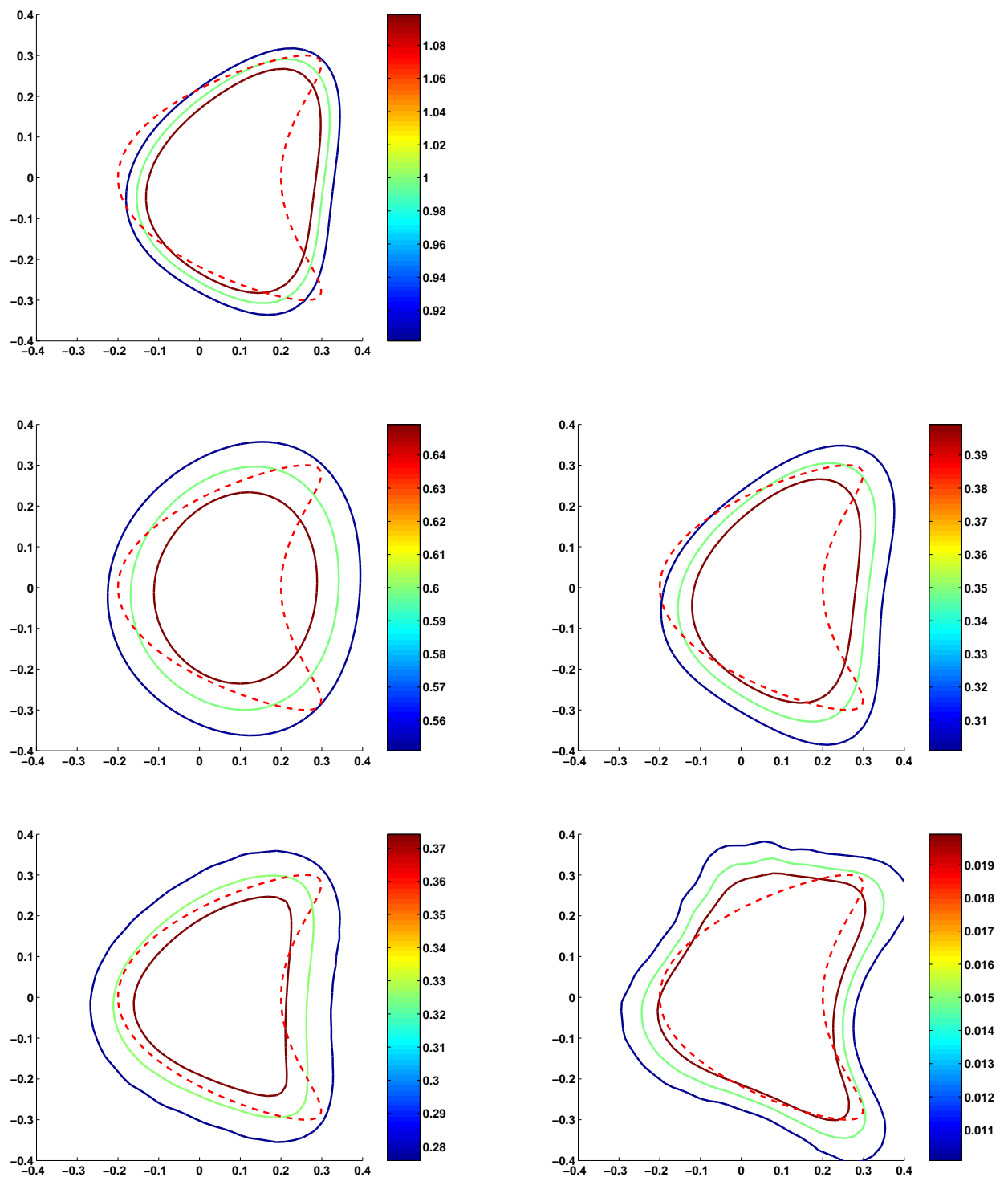

Fig. 8.13. Boundary reconstructions of the kite $20 \%$ noise. The first row shows the reconstruction with the indicator function of the LSM (left) The middle row illustrates the analogous reconstructions with the FFM (left) but additionally the reconstruction based on the map $z \mapsto \alpha(z)$. The last row shows the boundary reconstruction with the indicator function of the $\mathrm{SSM}$ (left) and the function $\mathcal{I}_{\text {enl }}$ (right). The regularization parameter was chosen with the generalized discrepancy principle with $\delta=0.2$ for the SSM and FM. For the regularization of the SSM we chose the value $\alpha=10^{-2}$. The obstacle is indicated with a dashed line. The wave number in this example was $\kappa=2.0$. 

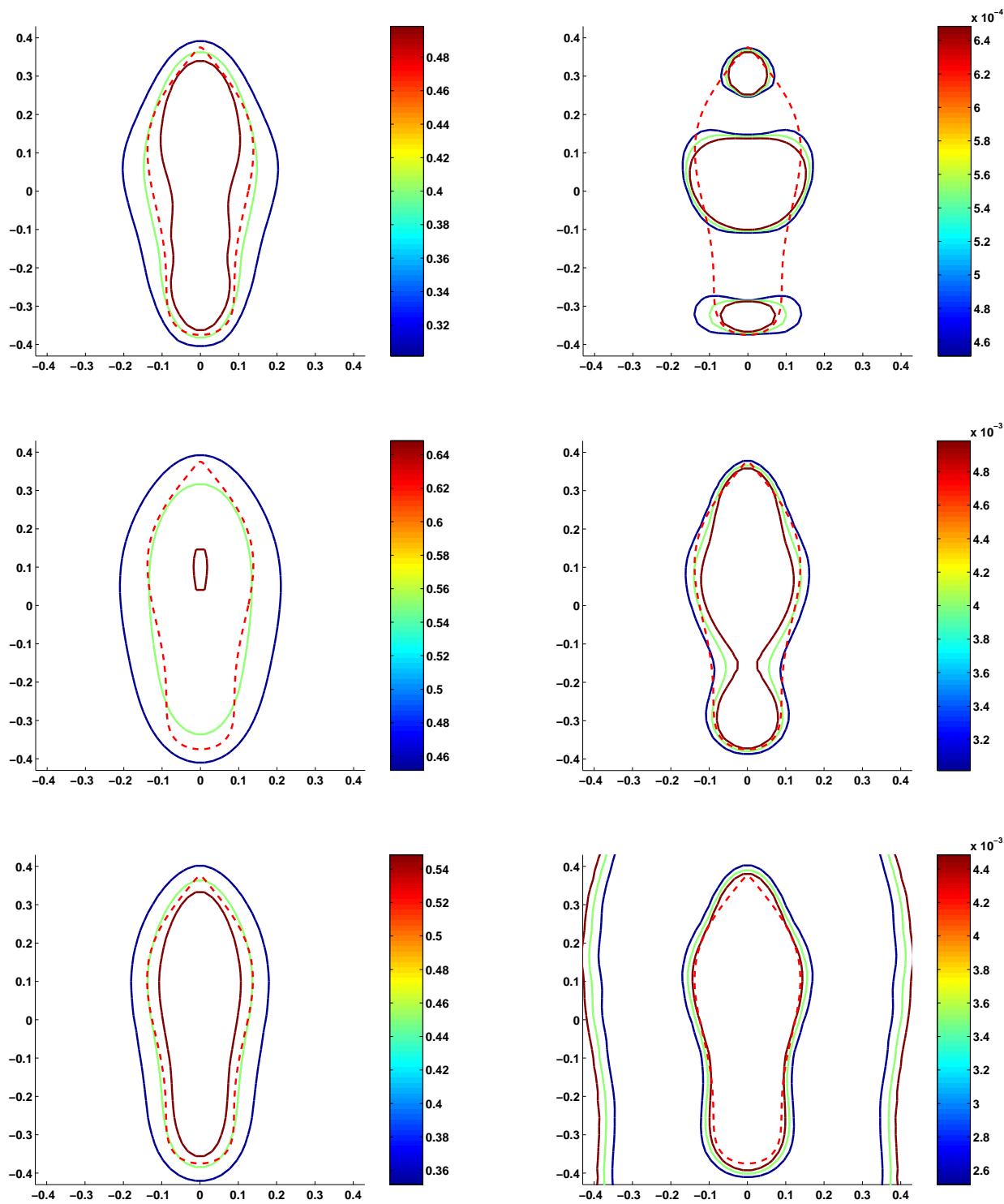

Fig. 8.14. Boundary reconstructions of the boat without noise. The first row shows the reconstruction with the indicator function of the LSM (left) and with the map $z \mapsto \alpha(z)$ as indicator function (right). The middle row illustrates the analogous reconstructions with the FFM while the last row shows the boundary reconstruction with the indicator function of the SSM (left) and the function $\mathcal{I}_{\text {enl }}$ (right). The regularization parameter was chosen with the generalized discrepancy principle with $\delta=0.01$ for the SSM and FM. For the regularization of the SSM we chose the value $\alpha=10^{-8}$. The obstacle is indicated with a dashed line. The wave number in this example was $\kappa=2.0$. 

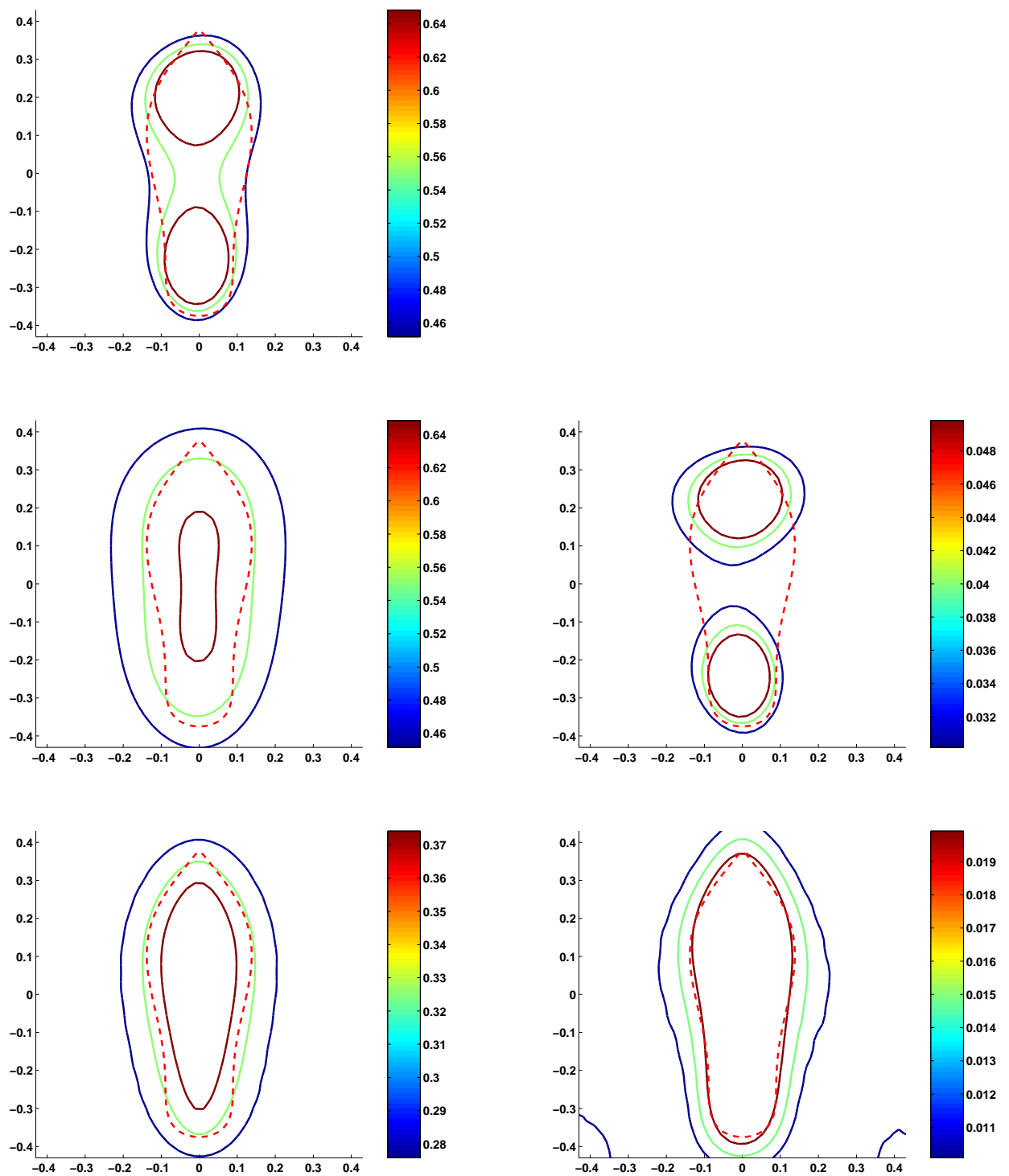

Fig. 8.15. Boundary reconstructions of the boat $5 \%$ noise. The first row shows the reconstruction with the indicator function of the LSM (left) The middle row illustrates the analogous reconstructions with the FFM (left) but additionally the reconstruction based on the map $z \mapsto \alpha(z)$. The last row shows the boundary reconstruction with the indicator function of the SSM (left) and the function $\mathcal{I}_{\text {enl }}$ (right). The regularization parameter was chosen with the generalized discrepancy principle with $\delta=0.05$ for the SSM and FM. For the regularization of the SSM we chose the value $\alpha=10^{-2}$. The obstacle is indicated with a dashed line. The wave number in this example was $\kappa=2.0$. 

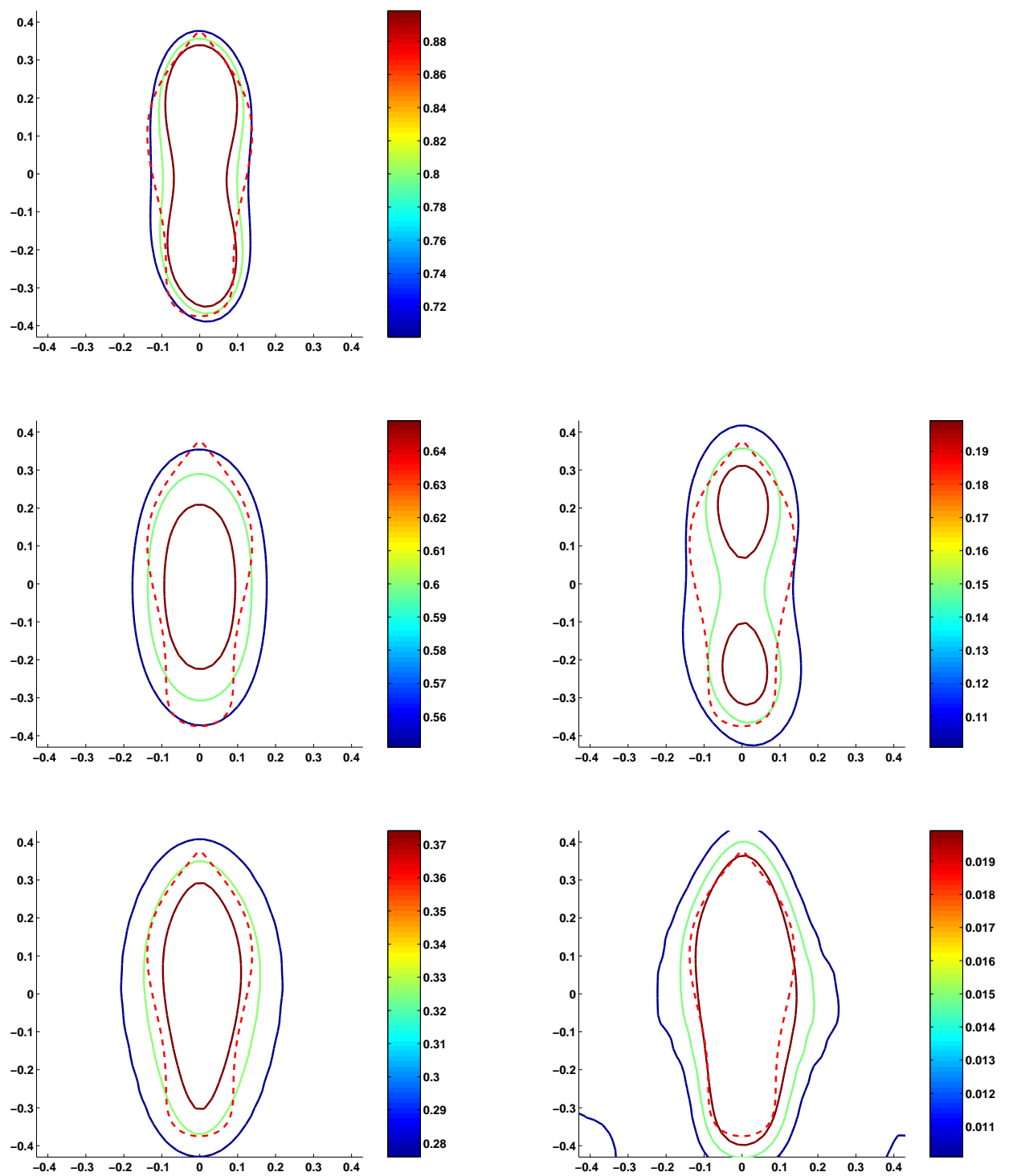

Fig. 8.16. Boundary reconstructions of the boat $10 \%$ noise. The first row shows the reconstruction with the indicator function of the LSM (left) The middle row illustrates the analogous reconstructions with the FFM (left) but additionally the reconstruction based on the map $z \mapsto \alpha(z)$. The last row shows the boundary reconstruction with the indicator function of the SSM (left) and the function $\mathcal{I}_{\text {enl }}$ (right). The regularization parameter was chosen with the generalized discrepancy principle with $\delta=0.1$ for the SSM and FM. For the regularization of the SSM we chose the value $\alpha=10^{-2}$. The obstacle is indicated with a dashed line. The wave number in this example was $\kappa=2.0$. 

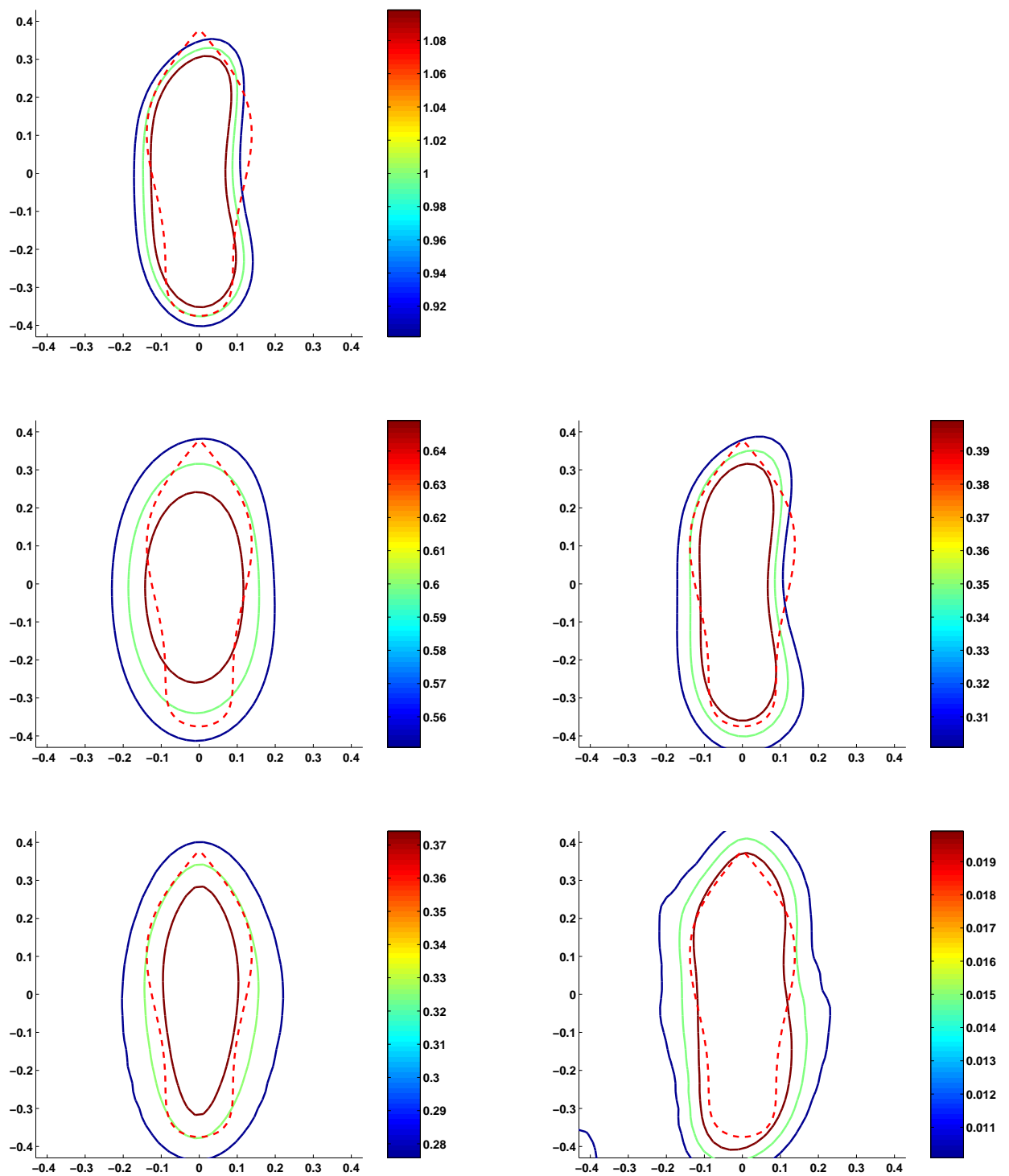

Fig. 8.17. Boundary reconstructions of the boat $20 \%$ noise. The first row shows the reconstruction with the indicator function of the LSM (left) The middle row illustrates the analogous reconstructions with the FFM (left) but additionally the reconstruction based on the map $z \mapsto \alpha(z)$. The last row shows the boundary reconstruction with the indicator function of the $\mathrm{SSM}$ (left) and the function $\mathcal{I}_{\text {enl }}$ (right). The regularization parameter was chosen with the generalized discrepancy principle with $\delta=0.2$ for the SSM and FM. For the regularization of the SSM we chose the value $\alpha=10^{-2}$. The obstacle is indicated with a dashed line. The wave number in this example was $\kappa=2.0$. 


\subsection{Numerical Examples of the SSM in $\mathbb{R}^{3}$}

In Chapter 6 we applied the point source method to the inverse acoustic scattering problem in $\mathbb{R}^{3}$. To this end we used the boundary integral equation method for the computation of the far field pattern of one incident wave. Of course, the input data of the SSM can be obtained from this forward solver in a straight forward way. We refer to Chapter 6 for the details of the numerical implementation and turn to a presentation of reconstruction examples for the SSM immediately.

In addition to the ball and the ring we will also reconstruct a T-shaped obstacle shown in Figure 8.18 together with its adapted approximation domain. Before we reconstruct the obstacle we will first compare the indicator
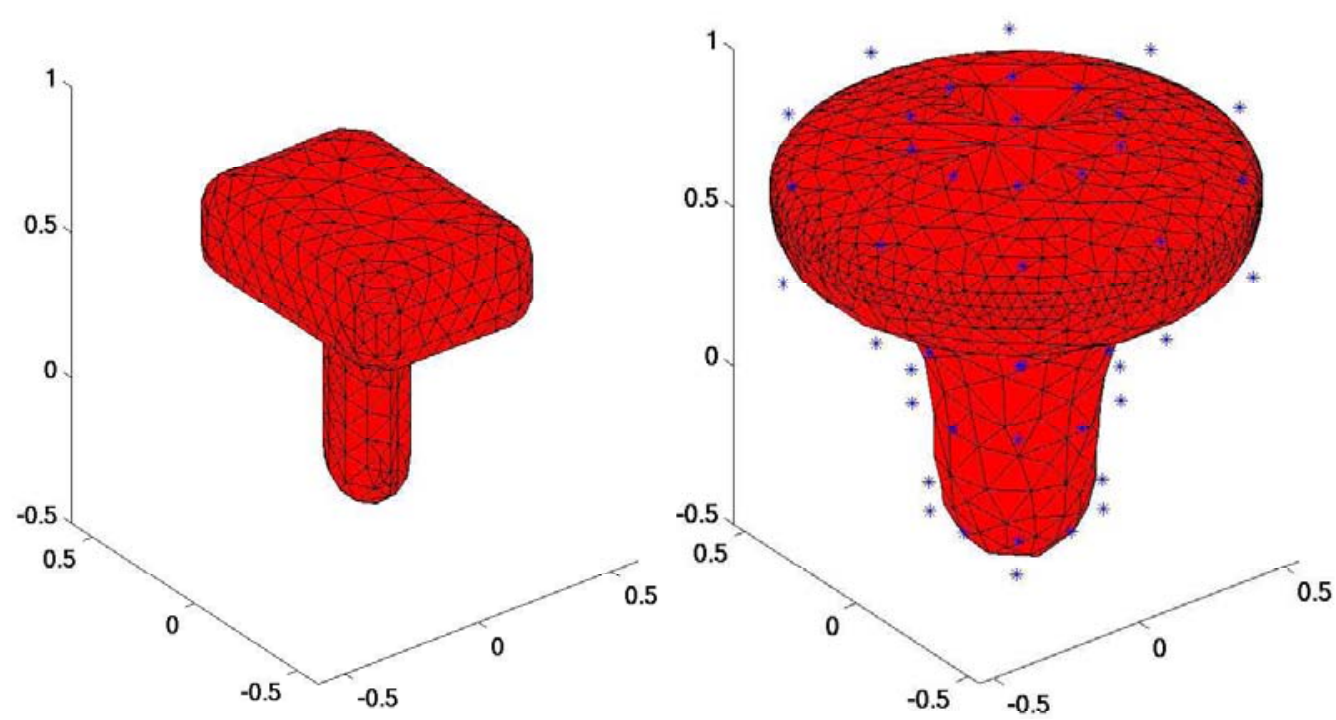

Fig. 8.18. The left figure shows the triangulation of the T-shaped obstacle with 1374 triangles. On the right side the adapted approximation domain which we use for the reconstruction is drawn. Each dot corresponds to the position $z_{0}^{(j)}$ of the source point for one fixed reference configuration $\left(z_{0}\left((j), G\left(z_{0}^{(j)}\right), j=1, \ldots, 62\right.\right.$.

function $I(z)=\Phi^{s}(z, z)$ of the SSM with the approximation

$$
\tilde{I}_{\mathrm{PSM}}(z):=4 \pi \int_{\mathbb{S}^{2}} \Phi^{\infty}(-d, z) g_{z, \varepsilon}(d) d s(d), \quad z \in \mathcal{B} .
$$

Note that $\tilde{I}_{\mathrm{PSM}}$ is the reconstruction of the scattered field $\Phi^{s}(\cdot, z)$ of an incident point source $\Phi(\cdot, z)$ from its far field pattern $\Phi^{\infty}(\cdot, z)$ with the PSM. Figure 8.19 illustrates the indicator function of the SSM whereas Figure 8.20 demonstrates the quality of the approximation $\tilde{I}_{\mathrm{PSM}}$ of the indicator function using the PSM. The wave number in this example was $\kappa=2$. For 

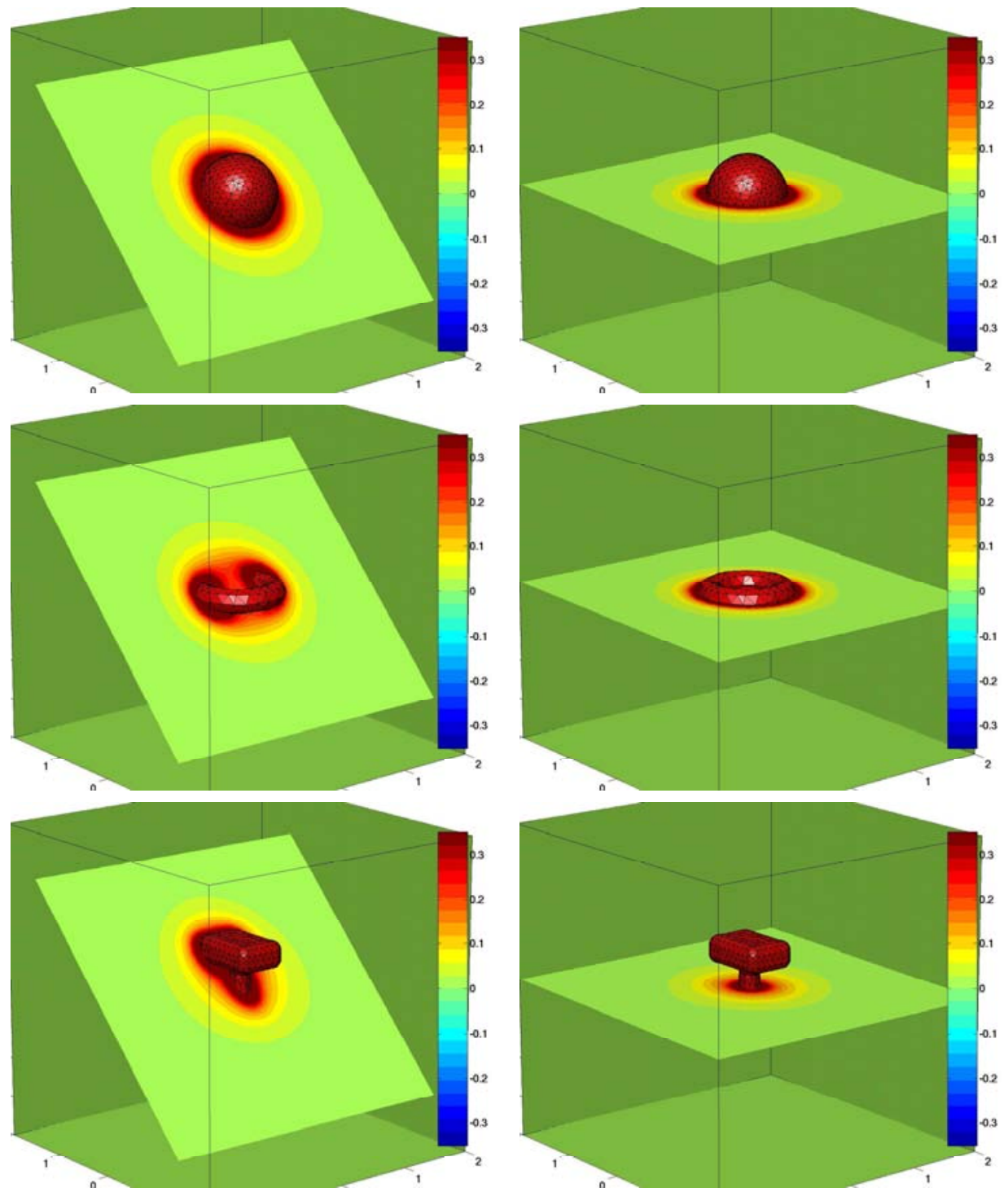

Fig. 8.19. The figure shows the modulus of the scattered field $\Phi^{s}(z, z)$ of incident point sources $\Phi(\cdot, z)$ evaluated in the source point itself. Each row shows two different slices of the rendered volume. The wave number of this example was $\kappa=2$.

the reconstruction with the PSM we used adapted approximation domains. In particular we reconstructed the scattered field for the ball with the spherical domains (6.131) of radius $r=0.7$, for the ellipsoid we approximated the point source on the ellipsoidal domains (6.133) and for the T-shaped obstacle we chose the 62 adapted approximation domains as illustrated in Figure 8.18 . The approximated enlighted area $\mathcal{E}_{c}$ was determined by the cut-off value $c=0.01$ and the control parameter $q=0.7$ as demonstrated in Chapter 6. Finally, a complete reconstruction is produced with (6.108) using the exponential weight functions (6.106) and (6.107) with the parameter $\tau=40$. The regularization parameter for this numerical example was 

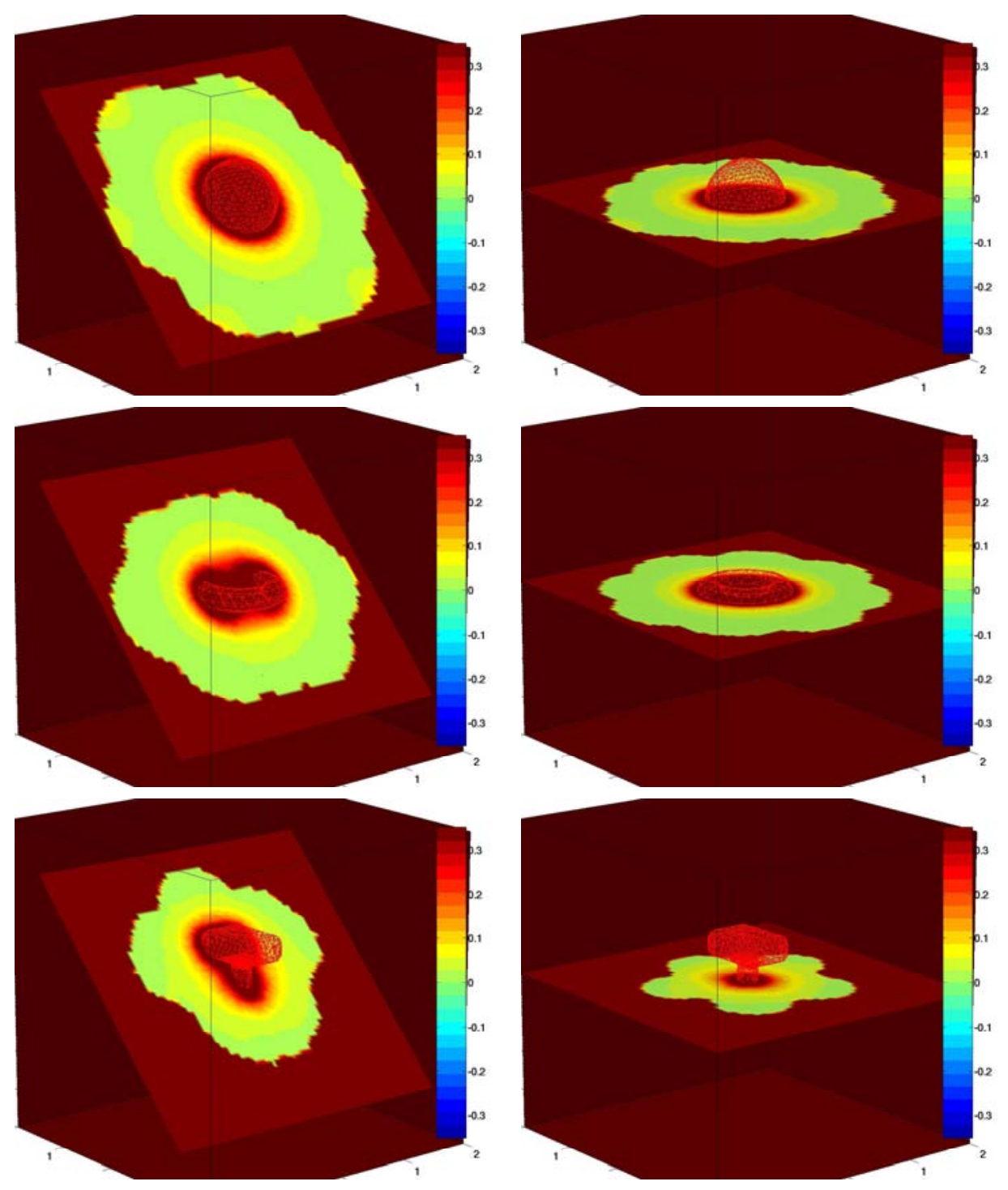

Fig. 8.20. The figure shows the modulus of the reconstructed scattered field $\Phi_{\mathrm{rec}}^{s}(z, z)$ of incident point sources $\Phi(\cdot, z)$ evaluated in the source point itself. For the reconstruction we applied the point source method with adapted approximation domains. The wave number of this example was $\kappa=2$.

$\alpha=10^{-8}$. Figure 8.20 demonstrates that the indicator function $\tilde{I}_{\mathrm{PSM}}$ yields a better approximation to the indicator function $I$ of the SSM for the ball and the T-shaped obstacle than for the ring. This is due to the fact that our adapted ellipsoidal approximation domains do not take care about the hole of the ring.

Instead of using the far field patterns $\Phi^{\infty}(\cdot, z)$ of scattered point waves we will now illustrate the approximating indicator function $\tilde{I}$ of the SSM itself, see Figure 8.21. The latter is based on the knowledge of the far field patterns of all incident plane waves and includes a second application of the PSM and hence a further approximation which we already pointed out be- 
fore. Although we increase the cut-off value for the approximated enlighted
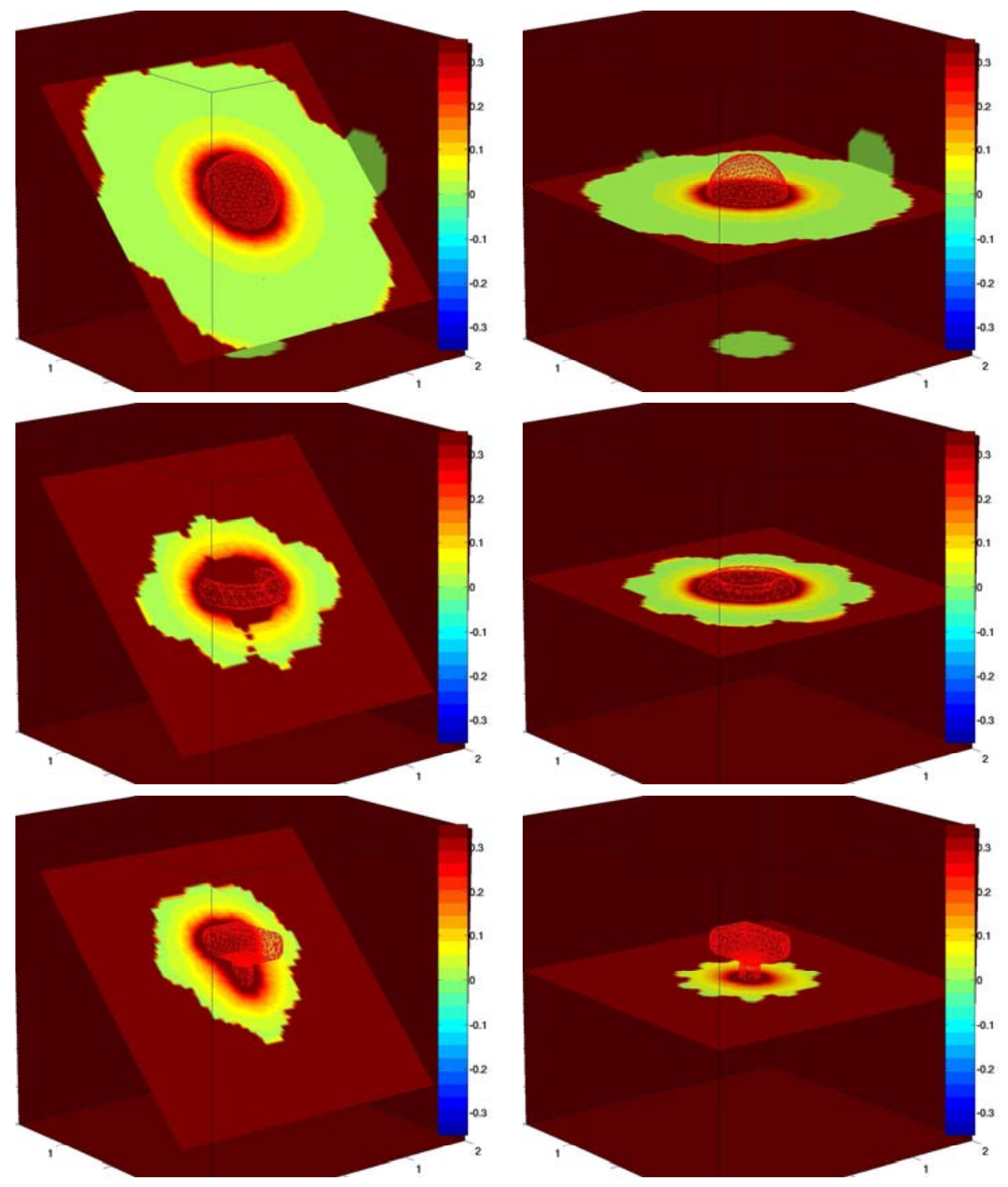

Fig. 8.21. The figure shows the modulus of the reconstructed scattered field $\Phi_{\mathrm{rec}}^{s}(z, z)$ of incident point sources $\Phi(\cdot, z)$ evaluated in the source point itself. For the reconstruction we applied the singular sources method with adapted approximation domains. The wave number of this example was $\kappa=2$.

areas $\mathcal{E}_{c}$ to $c=0.05$ for the reconstruction with the SSM, the approximated admissibility region becomes smaller for both the ring and the T-shaped obstacle. In particular, we can not expect to reconstruct the hole of the ring with the ellipsoidal approximation domains accurately. Once again, the simplest object - the ball - served for a calibration of the method. To this end we extract the 0.45 -levelset from the indicator function and obtain a boundary reconstruction of the ball as illustrated in Figure 8.22. Then, we reconstruct the other two obstacles as the 0.45 -levelset of the indicator func- 

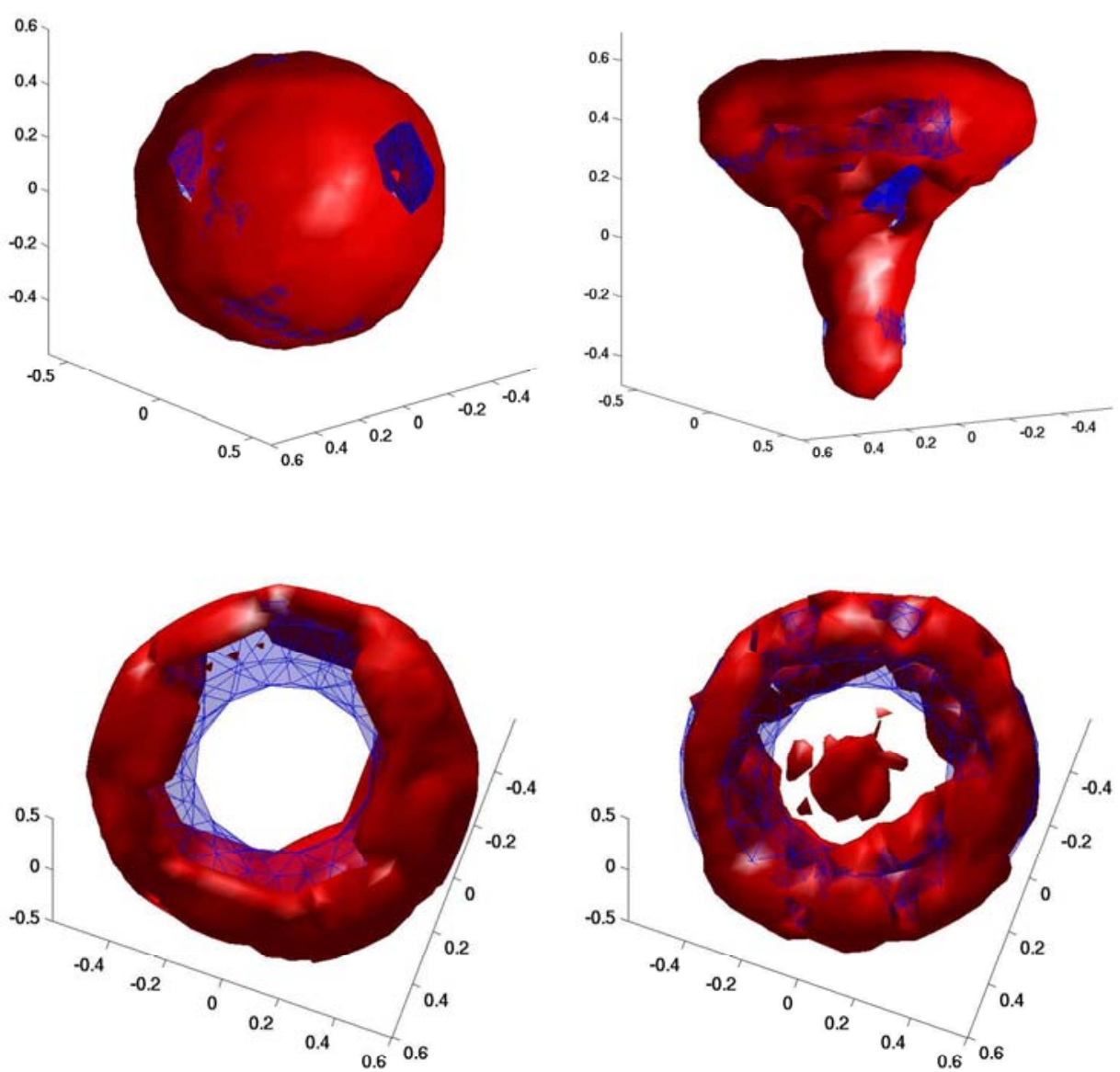

Fig. 8.22. The figure shows the boundary reconstruction of the ball, the ring and the Tshaped obstacle. For the reconstruction we applied the singular sources method with adapted approximation domains. The ring is reconstructed both with ellipsoidal approximation domains (left) and with approximation domains in form of a ring (right). The wave number of this example was $\kappa=2$.

tion. We remark that the ellipsoidal approximation domains are not suitable for the ring and expect an improved reconstruction with better adapted approximation domains. To this end we finally reconstruct the ring with the approximation domain $G^{(j)}=G$ given by a similar ring (6.118) with center radius $c=0.45$ and the radius $a=0.25$ of the tube. The source points $z^{(j)}$ of the configurations $\left(z^{(j)}, G\right)$ are located around the approximation domain $G$ at 60 positions

$$
z^{(j)}=z_{l k}:=R^{k}\left(0.45+0.25 \cdot \cos \frac{2 \pi l}{6}, 0,0.25 \cdot \sin \frac{2 \pi l}{6}\right)^{t}
$$

for $l=1, \ldots, 6, \quad k=1, \ldots, 10$, where 


$$
R=\left(\begin{array}{ccc}
\cos \frac{2 \pi}{10} & \sin \frac{2 \pi}{10} & 0 \\
-\sin \frac{2 \pi}{10} & \cos \frac{2 \pi}{10} & 0 \\
0 & 0 & 1
\end{array}\right)
$$

is a rotation matrix of angle $\frac{2 \pi}{10}$ around the $e_{3}$ axis. Figure 8.22 compares both reconstructions of the ring with the ellipsoidal (left) and with the ringshaped approximation domains (right). For the latter we illustrated the isosurface of level 0.55 . Figure 8.23 compares the aproximated admissibility regions of value $c=0.01$ and tells us, where to trust the reconstructions. In particular, the ring-shaped domains admit a reconstruction of the hole
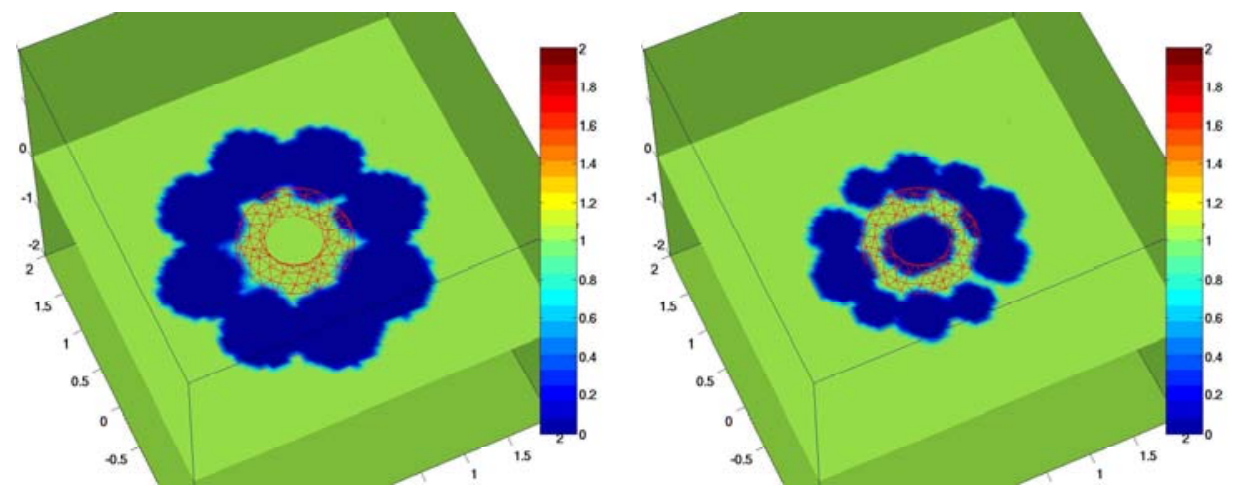

Fig. 8.23. The figure illustrates the union of the 26 areas $\mathcal{E}_{c}^{(j)}$ for the ellipsoidal (left) and the union of the 60 areas $\mathcal{E}_{c}^{(j)}$ for the ring-shaped approximation domain (right). The cut-off value was $c=0.01$.

of the scatterer while the ellipsoidal domains fail to approximate the indicator function of the SSM in the hole of the ring. Once again, this example demonstrates the advantage of the application of the indicator function $\mathcal{I}_{\text {enl }}$ to determine an approximated admissibility region from which we can judge the validity of the reconstruction.

As for the PSM we demonstrated the applicability of the SSM in $\mathbb{R}^{3}$ and reconstructed different geometrical obstacles with a two-step reconstruction algorithm. With a suitable choice of adapted approximation domains we were able to reconstruct nonconvex geometrical obstacles such as the ring and the T-shaped obstacle. The improvement of this method by using adapted approximation domains becomes apparent when we consider the indicator function of the admissibility region. With this additional indicator we obtain a measure for the validity of the reconstruction. In the reconstruction example of the ring, the indicator function told us that we cannot see the hole of the ring with the ellipsoidal approximation domains. On the other hand the indicator function also told us to trust the reconstruction obtained with the adapted ring-shaped approximation domains. Embedded in a two-step reconstruction algorithm the indicator function of 
the admissibility region provides helpful additional information on the validity of the reconstruction. The presented two-step reconstruction algorithms for point source approximation methods using adapted approximation domains provide a complete and competitive reconstruction of the unknown obstacles and enable further comparisons with reconstruction methods in inverse obstacle reconstruction problems. 


\section{Glossary}

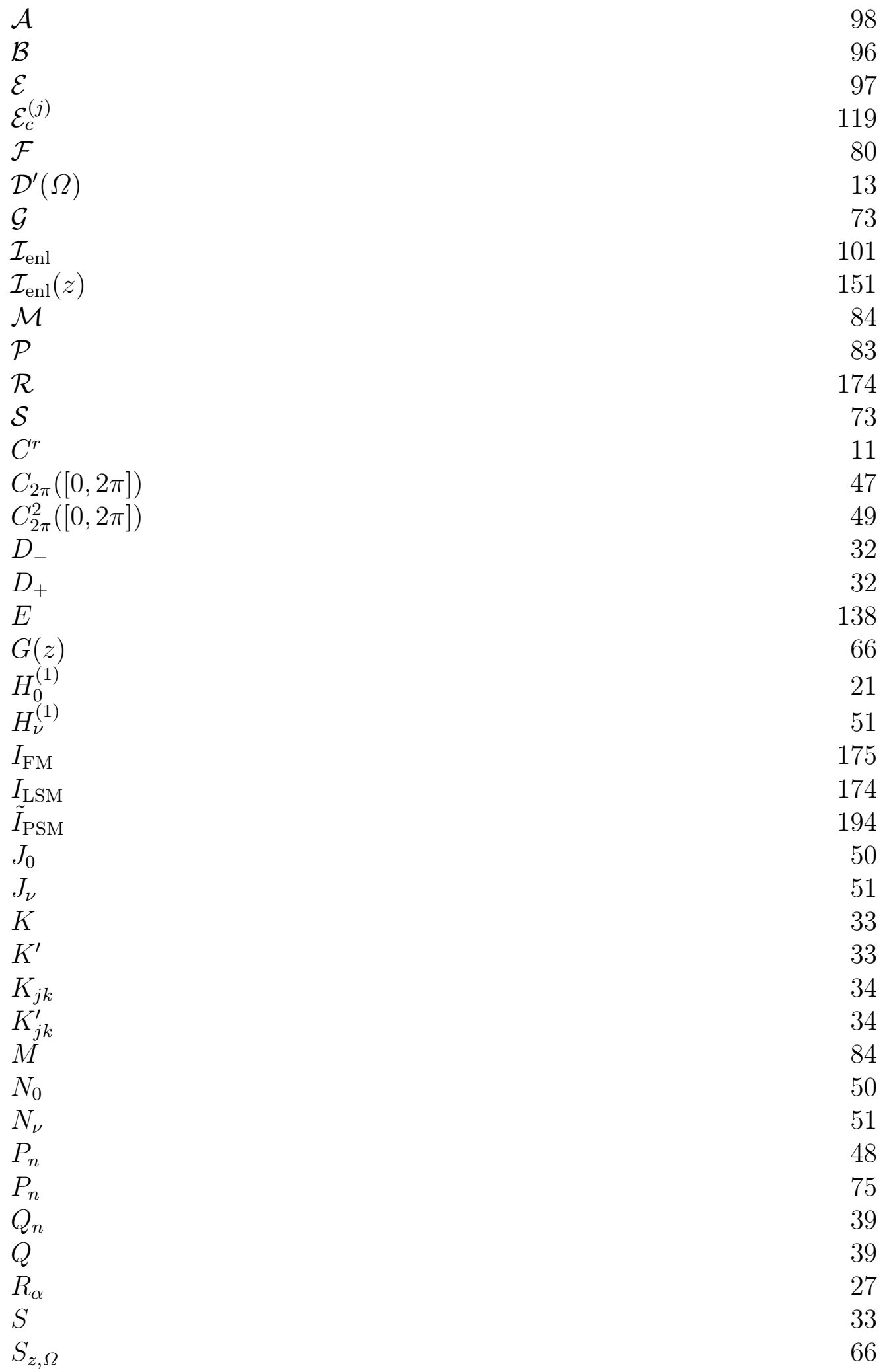


$S_{j k}$ 33

$\mathrm{SO}(m, \mathbb{R})$

$\Phi(x, y)$

$\Phi(x, y)$

$\Phi^{s}(x, z)$

107

$\Phi^{\infty}(\hat{x}, z)$

107

$\Gamma$

33

$\Omega$

$f_{ \pm}$

32

$\frac{\partial f_{ \pm}}{\partial \nu}$

32

$\frac{\partial u_{ \pm}}{\partial \nu}$

32

$u$

106

$u^{i}$

105

$u^{i}(x, d)$

107

$u^{s}$

105

$u^{s}(x, d)$

107

$y^{\delta}$

$y^{\delta}$

$u^{\delta} j, l$

28

28

$u_{j, l}^{\infty}$

$u_{j, l}^{\infty, \delta}$

176

176

177

$u^{\infty}$

106

$u^{\infty}(\hat{x}, d)$

107

$v_{ \pm}$

32

$v_{z}$

65

$\alpha$

28

$\alpha(\delta)$

29

$\delta_{a}$

13

$\lambda$

75

$\omega_{m-1}$

75

$\omega_{\tau}^{(j)}(z)$

119

$\tilde{\omega}_{\tau}^{(j)}(z)$

119 


\section{References}

1. Giovanni Alessandrini and Luca Rondi. Determining a sound-soft polyhedral scatterer by a single far-field measurement. Proc. Amer. Math. Soc., 133(6):1685-1691, 2005.

2. Philip M. Anselone. Collectively compact operator approximation theory and applications to integral equations. Prentice-Hall Inc., Englewood Cliffs, N. J., 1971. With an appendix by Joel Davis, Prentice-Hall Series in Automatic Computation.

3. Helmut Brakhage. Über die numerische Behandlung von Integralgleichungen nach der Quadraturformelmethode. Numer. Math., 2:183-196, 1960.

4. J. Cheng, J. Liu, and G. Nakamura. The numerical realization of the probe method for the inverse scattering problems from the near-field data. Inverse Problems, 21:839-855, 2005.

5. J. Cheng, J. Liu, and G. Nakamura. Recovery of boundaries and types for multiple obstacles from the far-field pattern, to appear.

6. David Colton and Andreas Kirsch. A simple method for solving inverse scattering problems in the resonance region. Inverse Problems, 12(4):383-393, 1996.

7. David Colton and Rainer Kress. Inverse acoustic and electromagnetic scattering theory, volume 93 of Applied Mathematical Sciences. Springer-Verlag, Berlin, second edition, 1998.

8. David Colton, Michele Piana, and Roland Potthast. A simple method using Morozov's discrepancy principle for solving inverse scattering problems. Inverse Problems, 13(6):14771493, 1997.

9. David Colton and B. D. Sleeman. Uniqueness theorems for the inverse problem of acoustic scattering. IMA J. Appl. Math., 31(3):253-259, 1983.

10. David L. Colton and Rainer Kress. Integral equation methods in scattering theory. Pure and Applied Mathematics. John Wiley \& Sons Inc., New York, 1983. A Wiley-Interscience Publication.

11. Jean Dieudonné. History of functional analysis, volume 49 of North-Holland Mathematics Studies. North-Holland Publishing Co., Amsterdam, 1981. Notas de Matemática [Mathematical Notes], 77.

12. K. Erhard and R. Potthast. A numerical study of $3 \mathrm{~d}$ reconstructions for the helmholtz equation and maxwell's equations. to appear.

13. K. Erhard and R. Potthast. A numerical study of the probe method. to appear.

14. K. Erhard and Roland Potthast. The point source method for reconstructing an inclusion from boundary measurements in electrical impedance tomography and acoustic scattering. Inverse Problems, 19(5):1139-1157, 2003.

15. Gerald B. Folland. Introduction to partial differential equations. Princeton University Press, Princeton, NJ, second edition, 1995.

16. Ivar Fredholm. Sur une classe déquations fonctionnelles. Acta math., 27:365-390, 1903.

17. David Gilbarg and Neil S. Trudinger. Elliptic partial differential equations of second order. Classics in Mathematics. Springer-Verlag, Berlin, 2001. Reprint of the 1998 edition.

18. D. Gintides. Local uniqueness for the inverse scattering problem in acoustics via the faberkrahn inequality. Inverse Problems, 21:1195-1205, 2005.

19. Wolfgang Hackbusch. Integralgleichungen. Teubner Studienbücher Mathematik. [Teubner Mathematical Textbooks]. B. G. Teubner, Stuttgart, 1989. Theorie und Numerik. [Theory and numerics], Leitfäden der Angewandten Mathematik und Mechanik [Guides to Applied Mathematics and Mechanics], 68. 
20. David Hilbert. Grundzüge einer allgemeinen Theorie der linearen Integralgleichungen. Chelsea Publishing Company, New York, N.Y., 1953.

21. Masaru Ikehata. Reconstruction of an obstacle from the scattering amplitude at a fixed frequency. Inverse Problems, 14(4):949-954, 1998.

22. Masaru Ikehata. Reconstruction of the shape of the inclusion by boundary measurements. Comm. PDE, 23:1459-1474, 1998.

23. Masaru Ikehata. Enclosing a polygonal cavity in a two-dimensional bounded domain from Cauchy data. Inverse Problems, 15(5):1231-1241, 1999.

24. Masaru Ikehata. Reconstruction of a source domain from the Cauchy data. Inverse Problems, 15(2):637-645, 1999.

25. Masaru Ikehata. Reconstruction of obstacle from boundary measurements. Wave Motion, 30(3):205-223, 1999.

26. V. Isakov. On uniqueness in the inverse transmission scattering problem. Inverse Problems, 9:535-543, 1993.

27. Victor Isakov. On uniqueness in the inverse transmission scattering problem. Comm. Partial Differential Equations, 15(11):1565-1587, 1990.

28. Oliver Dimon Kellogg. Foundations of potential theory. Reprint from the first edition of 1929. Die Grundlehren der Mathematischen Wissenschaften, Band 31. Springer-Verlag, Berlin, 1967.

29. Heinrich Kersten. Grenz- und Sprungrelationen für Potentiale mit quadratsummierbarer Flächenbelegung. Resultate Math., 3(1):17-24, 1980.

30. A. Kirsch and R. Kress. Uniqueness in inverse obstacle scattering. Inverse Problems, 9:285299, 1993.

31. Andreas Kirsch. An introduction to the mathematical theory of inverse problems, volume 120 of Applied Mathematical Sciences. Springer-Verlag, New York, 1996.

32. Andreas Kirsch. Characterization of the shape of a scattering obstacle using the spectral data of the far field operator. Inverse Problems, 14(6):1489-1512, 1998.

33. Rainer Kress. Linear integral equations, volume 82 of Applied Mathematical Sciences. Springer-Verlag, Berlin, 1989.

34. Rainer Kreß. Fast 100 Jahre Fredholmsche Alternative. In Jahrbuch Überblicke Mathematik, 1994, pages 14-27. Vieweg, Braunschweig, 1994.

35. Rainer Kress. On the numerical solution of a hyper-singular integral equation in scattering theory. J. Comput. Appl. Math., 61(3):345-360, 1995.

36. Rainer Kress. Linear integral equations, volume 82 of Applied Mathematical Sciences. Springer-Verlag, New York, second edition, 1999.

37. Rainer Kress. Electromagnetic waves scattering: Specific theoretical tools. In Pike and Sabatier, editors, Scattering, pages 175-190. Academic Press, London, 2001.

38. Peter D. Lax and Ralph S. Phillips. Scattering theory. Pure and Applied Mathematics, Vol. 26. Academic Press, New York, 1967.

39. Henri Lebesgue. Integrale, Longueur, Aire. Annali di Mat., 7:231-358, 1902.

40. Henri Lebesgue. Leçons sur les séries trigonométriques. Librairie Scientifique et Technique Albert Blanchard, Paris, 1975. Professées au Collège de France, 1904-05, Nouveau tirage.

41. C. Liu and A. Nachman. A scattering theory analogue of a theorem of Polya and an inverse obstacle problem. In preparation.

42. J. Liu. Private communication with R. Potthast, 2002.

43. D. R. Luke and R. Potthast. The no response test - a sampling method for inverse scattering problems. SIAM J. Appl. Math., 63(4):1292-1312 (electronic), 2003.

44. A.-W. Maue. Zur Formulierung eines allgemeinen Beugungsproblems durch eine Integralgleichung. Z. Physik, 126:601-618, 1949.

45. S. G. Mikhlin. Integral equations and their applications to certain problems in mechanics, mathematical physics and technology. Pergamon Press, New York, 1957. Translated from the Russian by A. H. Armstrong.

46. S. G. Mikhlin. Mathematical physics, an advanced course. With appendices by V. M. Babič, V. G. Maz'ja and I. Ja. Bakel'man. Translated from the Russian. North-Holland Series in Applied Mathematics and Mechanics, Vol. 11. North-Holland Publishing Co., Amsterdam, 1970.

47. Claus Müller. Spherical harmonics, volume 17 of Lecture Notes in Mathematics. Springer, Berlin, 1966. 
48. G. Nakamura, R. Potthast, and M. Sini. Unification of the probe and singular sources methods for the inverse boundary value problem by the no-response test. Preprint.

49. Norbert Ortner. Regularisierte Faltung von Distributionen. I. Zur Berechnung von Fundamentallösungen. Z. Angew. Math. Phys., 31(1):133-154, 1980.

50. Norbert Ortner. Regularisierte Faltung von Distributionen. II. Eine Tabelle von Fundamentallösungen. Z. Angew. Math. Phys., 31(1):155-173, 1980.

51. Norbert Ortner and Peter Wagner. A survey on explicit representation formulae for fundamental solutions of linear partial differential operators. Acta Appl. Math., 47(1):101-124, 1997.

52. R. Potthast. A fast new method to solve inverse scattering problems. Inverse Problems, 12(5):731-742, 1996.

53. R. Potthast. A point source method for inverse acoustic and electromagnetic obstacle scattering problems. IMA J. Appl. Math., 61(2):119-140, 1998.

54. R. Potthast. Point sources and multipoles in inverse scattering theory. Habilitation thesis, Göttingen, 1999.

55. R. Potthast. Stability estimates and reconstructions in inverse acoustic scattering using singular sources. J. Comput. Appl. Math., 114(2):247-274, 2000.

56. R. Potthast. On the convergence of a new newton-type method in inverse scattering. Inverse Problems, 17:1419-1434, 2001.

57. R. Potthast. Point sources and multipoles in inverse scattering theory, volume 427 of Chapman $\&$ Hall/CRC Research Notes in Mathematics. Chapman \& Hall/CRC, Boca Raton, FL, 2001.

58. R. Potthast. On the convergence of the no response test. Submitted for publication.

59. R. Potthast. A new non-iterative singular sources method for the reconstruction of piecewise constant media. Numerische Mathematik, to appear.

60. R. Potthast. Sampling and probe methods - an algorithmical view. Computing, to appear.

61. R. Potthast and J. Schulz. A multi-wave range test for obstacle reconstruction. submitted for publication.

62. R. Potthast and I. Stratis. The singular sources method for an inverse transmission problem. Computing, to appear.

63. R. Potthast, John Sylvester, and Steven Kusiak. A 'range test' for determining scatterers with unknown physical properties. Inverse Problems, 19(3):533-547, 2003.

64. Franz Rellich. Über das asymptotische Verhalten der Lösungen von $\Delta u+\lambda u=0$ in unendlichen Gebieten. Jber. Deutsch. Math. Verein., 53:57-65, 1943.

65. Frigyes Riesz. Untersuchungen über Systeme integrierbarer Funktionen. Math. Ann., 69:449-497, 1910.

66. Frigyes Riesz. über lineare Funktionalgleichungen. Acta Math., 41:71-98, 1918.

67. Frigyes Riesz. Oeuvres complètes. (Title also in Hungarian and German). Publiées sur l'ordre de l'Académie des Sciences de Hongrie par Ákos Császár. 2 Vols. Akadémiai Kiadó, Budapest, 1960.

68. Steven H. Schot. Eighty years of Sommerfeld's radiation condition. Historia Math., 19(4):385-401, 1992.

69. Laurent Schwartz. Théorie des distributions. Publications de l'Institut de Mathématique de l'Université de Strasbourg, No. IX-X. Nouvelle édition, entiérement corrigée, refondue et augmentée. Hermann, Paris, 1966.

70. Arnold Sommerfeld. Die Greensche Funktion der Schwingungsgleichung. Jahresbericht der DMV , 21:309-353, 1912.

71. Plamen Stefanov and Gunther Uhlmann. Local uniqueness for the fixed energy fixed angle inverse problem in obstacle scattering. Proc. Amer. Math. Soc., 132(5):1351-1354 (electronic), 2004.

72. Michael E. Taylor. Partial differential equations. I, volume 115 of Applied Mathematical Sciences. Springer-Verlag, New York, 1996. Basic theory.

73. François Trèves. Basic linear partial differential equations. Academic Press [A subsidiary of Harcourt Brace Jovanovich, Publishers], New York-London, 1975. Pure and Applied Mathematics, Vol. 62.

74. Michael von Renteln. Zur Situation der Analysis um die Jahrhundertwende. In Vorlesungen zum Gedenken an Felix Hausdorff, volume 5 of Berliner Studienreihe Math., pages 107-130. Heldermann, Berlin, 1994. 
75. Joachim Weidmann. Lineare Operatoren in Hilberträumen. B. G. Teubner, Stuttgart, 1976. Mathematische Leitfäden.

76. Wolfgang Wendland. Die Fredholmsche Alternative für Operatoren, die bezüglich eines bilinearen Funktionals adjungiert sind. Math. Z., 101:61-64, 1967.

77. Lutz Wienert. Die numerische Approximation von Randintegraloperatoren für die Helmholtzgleichung im $\mathbb{R}^{3}$. PhD thesis, Georg-August-Universität Göttingen, 1990. 


\section{Index}

a posteriori 103

a posteriori strategy 29

a priori strategy 29

acoustic scattering

bvp 91

acoustic scattering problem

direct 105

invers 107

adjoint 36

adjoint double-layer potential 33

adjoint operator 36, 66

admissibility condition 66

admissibility region 98

analytic continuation method 89

approximation domain

admissible 66

reference 80

strategy 74

backprojection operator $\quad 89,113$

Bessel function 21,50

boundary

of class $C^{r} \quad 11$

regularity 11

Chebychev polynomial 75

collectively compact 41

combined layer 106

combined layer potential 53

compact

collectively 41

compact operator 25

consistent discretization 40

convergence

weak 68

convergent discretization 41

Coulomb, Charles Augustin de

D-t-N map 144

decomposition method 89

density 31

descretization

consistent 40 dipole 31

moment 31

Dirac's delta distribution 13

Dirac, Paul Adrien Maurice 13

direct bvp 53

direct values 32

Dirichlet eigenvalue 23

Dirichlet problem

Helmholtz' equation

exterior 23

interior 22

Laplace's equation

exterior 19

interior 19

Dirichlet to Neumann map 144

discrepancy function 174

discrepancy principle 174

discretization

convergent 41

stable 41

dissipative wave equation 20

distribution 13

derivative 14

Dirac's delta 13

divergence theorem 11

domain

of interest 96

physical 96

domain of interest 65

double-layer potential $\quad 31,49,52$

dual system 36

eigenvalue 23

electrical impedance tomography bvp 91

enclosure method 141, 143

enlighted area 97

entire solution 74

equation

of the first kind 25

of the second kind 25

Euler constant 50

exterior Dirichlet problem 
Helmholtz' equation 23

Laplace's equation 19

exterior domain 32

exterior Neumann problem

Helmholtz' equation 23

Laplace's equation 19

factorization method 141,142

far field

equation 142

far field pattern 106

Fischer, Ernst 35

Fredholm's alternative 36

Fredholm, Erik Ivar 34

frequency 20

Funck-Hecke formula 108

function

harmonic 17

fundamental solution 14

of Helmholtz' equation 21

of Laplace operator $\quad 14,17$

of Laplace's equation 17

asymptotics 50

Funk-Hecke formula 75

Gauss, Johann Carl Friedrich 12

gravitiational field 16

Green's formula

first 12

second 12

Green's formulae 12

Green's representation formula 106

Green's representation theorem 17

Green, George 12

Hadamard, Jacques Salomon 27

Hankel function 15,21

Hankel funktion 50

harmonic function 17

Helmholtz representation theorem 21

Helmholtz' equation 20

Herglotz wave function 65, 74 static 75

Herglotz wave oeprator 74

Hilbert, David 34

Hilbert-Schmidt kernel 38

Holmgren's Theorem 92,95

Holmgren, Erik 34

ill-posed 27

ill-posed problem 27

impulse size condition 100

incident field 105

indicator functional 147

integral operator 26

interior Dirichlet problem

Helmholtz equation 22

Laplace's equation 19 interior domain 32

interior Neumann problem

Helmholtz' equation 22

Laplace's equation 19

jump relations

for $L^{2}$ densities 37

kernel 26

weakly singular 26

Klein, Felix 22

Lagrange basis 48

Laplace's equation 17

law of gravitation 16

Lebesgue, Henri 34

Legendre polynomial 75

linear sampling method $141,142,172$

logarithmic singularity 47

Malgrange-Ehrenpreis theorem 15

Maue's identity 145

maximum-minimum principle

strong 18

weak 18

mixed reciprocity relation $\quad 91,113,165$

Morozov's discrepancy principle 174

Neumann eigenvalue 23

Neumann function 50

Neumann problem

Helmholtz' equation

exterior 23

interior 22

Laplace's equation

exterior 19

interior 19

Newton, Isaac 15

no response test $100,141,142$

Nyström's method 39, 58

$$
\begin{aligned}
& \text { operator } \\
& \text { adjoint } 66 \\
& \text { backprojection } \\
& \text { adjoint } 36 \\
& \text { compact } 25 \\
& \text { integral } 26
\end{aligned}
$$

backprojection 89

physical domain 96

Picard's theorem 29

plane wave 21, 106

platonic bodies 85

Poincaré, Jules Henri 34

point charge 16, 30

point mass 16

point source 30, 107

point source method 89

multi-wave version 165

redesign 89 
potential

adjoint double-layer 33

combined layer 53

double-layer $31,49,52$

single-layer $30,49,51$

prime number theorem 27

principle of superposition 90

principle of uniform boundedness 68

probe method 141, 143

PSM 89

quadrature points 39

quadrature rule 39

convergent 39

quadrature weight 39

quadrature weights 48

radiating solution 106

radiation condition 21

range test 141,142

reconstructed field

by PSM 97

reconstructed scattered field

by PSM 111

reduced wave equation 20

regular

regularization scheme 29

regularity

of boundary 11

regularization paramter 28

regularization scheme 27

regular 29

strategy 29

relatively compact 25

Rellich's lemma 107

Rellich, Franz 22

repeated trapezoidal rule 39

representation theorem

for Helmholtz'equation 21

for Laplace's equation 17

response 101

Riesz' theorem 35

Riesz, Frigyes 34

Rodrigues' formula 75

sampling method 141

sampling point set of 73

sampling points 73

scattered field 105

scatterer

sound-soft 53

Schwartz, Laurent 13

semi-discrete 40

single-layer potential $\quad 30,49,51$

singular sources method 141,142

singular value decomposition 29

Sommerfeld radiation condition 21, 105

Sommerfeld, Arnold Johannes Wilhelm 22

sound-soft 53

stable discretization 41

stable solution 27

static Herglotz wave function 75

strategy 74

a posteriori 29

a priori 29

regularization scheme 29

strong maximum-minimum principle 18

superposition

of plane waves 74

theorem

Holmgren 92

divergence 11

Green's representation 17

Helmholtz' representation 21

Malgrange-Ehrenpreis 15

Riesz 35

Tikhonov regularization 29

total field 106

trapezoidal rule 39

repeated 39

trigonometric interpolation 48

uniform boundedness 68

wave equation 20

wave number 20

weak convergence 68

weak maximum-minimum principle 18

weakly singular kernel 26

weight function 39

well-posed 27 


\title{
Curriculum Vitae
}

\author{
Personal Data
}

Name: $\quad$ Klaus Erhard

Date of birth: $\quad$ 12.03.1975

Place of birth: Bad Kissingen

Name of parents: Lothar Erhard

Ingrid Erhard (birth name: Büttner)

\section{Primary and Secondary Education}

1981 - 1985 Elementary school, Oerlenbach

1985 - 1994 Secondary school, Gymnasium Bad Kissingen

\section{Tertiary Education}

11/1994 - 12/2001 study for a degree in mathematics and physics for teaching at secondary schools (Gymnasium), University of Würzburg

04/2001 diploma in mathematics

12/2001 diploma of the state (1. Staatsexamen) in physics and mathematics

\section{Occupational Career}

01/2002 - 10/2003 scientific employee at the department of numerical and applied mathematics, University of Göttingen

$11 / 2003-08 / 2005$ scientific employee in the BMBF-project „,Humanitäres Minenräumen und Metalldetektoren”

09/2005 - $\quad$ scientific employee at the Philips Medical Systems research laboratories, Hamburg 
Ich erkläre an Eides statt, dass die Dissertation „Point Source Approximation Methods in Inverse Obstacle Reconstruction Problems" selbständig und ohne unerlaubte Hilfe angefertigt wurde.

Göttingen, den 26. September 2005

Klaus Erhard 